\title{
Fundamentals of Quantum Information Theory
}

\author{
Michael Keyl ${ }^{1}$ \\ TU-Braunschweig, Inst. Math. Phys, Mendelssohnstraße 3, D-38106 Braunschweig
}

February 1, 2008

${ }^{1}$ electronic mail: M.Keyl@TU-BS.DE 


\begin{abstract}
In this paper we give a self contained introduction to the conceptional and mathematical foundations of quantum information theory. In the first part we introduce the basic notions like entanglement, channels, teleportation etc. and their mathematical description. The second part is focused on a presentation of the quantitative aspects of the theory. Topics discussed in this context include: entanglement measures, channel capacities, relations between both, additivity and continuity properties and asymptotic rates of quantum operations. Finally we give an overview on some recent developments and open questions.
\end{abstract}




\section{Contents}

$\begin{array}{llr}1 & \text { Introduction } & \mathbf{5}\end{array}$

1.1 What is quantum information? . . . . . . . . . . . . . . 5

1.2 Tasks of quantum information . . . . . . . . . . . . . . 8

1.3 Experimental realizations . . . . . . . . . . . . . . . 9

$\begin{array}{lll}2 & \text { Basic concepts } & \mathbf{1 1}\end{array}$

2.1 Systems, States and Effects . . . . . . . . . . . . . . . 11

2.1 .1 Operator algebras . . . . . . . . . . . . . . . . 11

2.1 .2 Quantum mechanic . . . . . . . . . . . . . . . . . 12

2.1.3 Classical probability . . . . . . . . . . . . . . . 13

2.1 .4 Observables . . . . . . . . . . . . . . . . . . 14

2.2 Composite systems and entangled states . . . . . . . . . . . . . 15

2.2.1 Tensor products .................... 15

2.2 .2 Compound and hybrid systems . . . . . . . . . . . . . . 16

2.2.3 Correlations and entanglement . . . . . . . . . . . . . . . 17

2.2 .4 Bell inequalities. . . . . . . . . . . . . . . . . . . . . 18

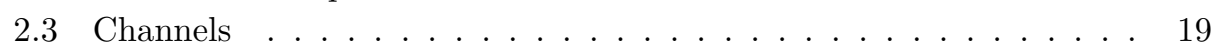

2.3 .1 Completely positive maps . . . . . . . . . . . . . . . 19

2.3 .2 The Stinespring theorem . . . . . . . . . . . . . . . 20

2.3 .3 The duality lemma . . . . . . . . . . . . . . . . . . . 21

2.4 Separability criteria and positive maps . . . . . . . . . . . . . . 21

2.4 .1 Positivity . . . . . . . . . . . . . . . . . . . 22

2.4 .2 The partial transpose . . . . . . . . . . . . . . . . 22

2.4 .3 The reduction criterion . . . . . . . . . . . . . 23

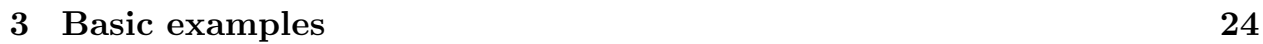

3.1 Entanglement . . . . . . . . . . . . . . . . . . . . . 24

3.1.1 Maximally entangled states . . . . . . . . . . . . . . . . 24

3.1.2 Werner states . . . . . . . . . . . . . . . . 25

3.1 .3 Isotropic states . . . . . . . . . . . . . . . . . 26

3.1.4 OO-invariant states . . . . . . . . . . . . . . 27

3.1 .5 PPT states . . . . . . . . . . . . . . . . . . . 29

3.1 .6 Multipartite states . . . . . . . . . . . . . . . . . 29

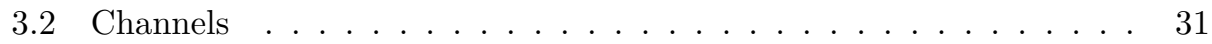

3.2.1 Quantum channnels . . . . . . . . . . . . . . . . 31

3.2.2 Channels under symmetry . . . . . . . . . . . . . . . . . . 32

3.2.3 Classical channels . . . . . . . . . . . . . . . . . . . 33

3.2.4 Observables and Preparations . . . . . . . . . . . . . . 34

3.2 .5 Instruments and Parameter Dependent Operations . . . . . . 34

3.2 .6 LOCC and separable channels . . . . . . . . . . . . 36

3.3 Quantum mechanics in phase space . . . . . . . . . . . . . . 37

3.3.1 Weyl operators and the CCR . . . . . . . . . . . . . . 38

3.3.2 Gaussian states . . . . . . . . . . . . . . . . . . . . . . . 39

3.3.3 Entangled Gaussians . . . . . . . . . . . . . . . . . . . . . . . 39

3.3.4 Gaussian channels ................... . . 41 


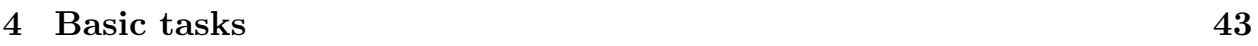

4.1 Teleportation and dense coding . . . . . . . . . . . . . . . . . . 43

4.1 .1 Impossible machines revisited: Classical teleportation . . . . 43

4.1.2 Entanglement enhanced teleportation . . . . . . . . . . 43

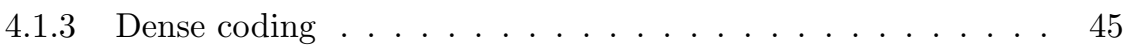

4.2 Estimating and copying . . . . . . . . . . . . . . . . 46

4.2.1 Quantum state estimation . . . . . . . . . . . . . . . 46

4.2 .2 Approximate cloning . . . . . . . . . . . . . . . 47

4.3 Distillation of entanglement . . . . . . . . . . . . . . . . . . . 48

4.3 .1 Distillation of pairs of qubits . . . . . . . . . . . . . 49

4.3 .2 Distillation of isotropic states . . . . . . . . . . . . . . 50

4.3.3 Bound entangled states . . . . . . . . . . . . . . 50

4.4 Quantum error correction . . . . . . . . . . . . . . . 51

4.5 Quantum computing .................... . . . 53

4.5.1 The network model of classical computing . . . . . . . . . . . 53

4.5 .2 Computational complexity . . . . . . . . . . . . . . 54

4.5.3 Reversible computing . . . . . . . . . . . . . . . . 55

4.5.4 The network model of a quantum computer . . . . . . . . . . 56

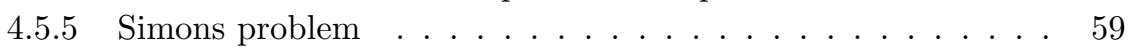

4.6 Quantum cryptography . . . . . . . . . . . . . . . 59

5 Entanglement measures $\quad 62$

5.1 General properties and definitions . . . . . . . . . . . . . . 62

5.1 .1 Axiomatics . . . . . . . . . . . . . . . . 62

5.1 .2 Pure states . . . . . . . . . . . . . . . . . . 64

$5.1 .3 \quad$ Entanglement measures for mixed states . . . . . . . . . . . . 65

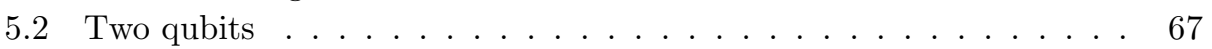

5.2 .1 Pure states . . . . . . . . . . . . . . . . 67

5.2 .2 EOF for Bell diagonal states . . . . . . . . . . . . . . . . 68

$5.2 .3 \quad$ Wootters formula . . . . . . . . . . . . . . . 69

$5.2 .4 \quad$ Relative entropy for Bell diagonal states . . . . . . . . . . . . 70

5.3 Entanglement measures under symmetry . . . . . . . . . . . . . . . 71

5.3.1 Entanglement of Formation . . . . . . . . . . . . . . . . 71

$5.3 .2 \quad$ Werner states . . . . . . . . . . . . . . . . 71

$5.3 .3 \quad$ Isotropic states . . . . . . . . . . . . . . . . . . . . . . . 72

$5.3 .4 \quad$ OO-invariant states . . . . . . . . . . . . . . . . . 74

$5.3 .5 \quad$ Relative Entropy of Entanglement . . . . . . . . . . . . . . . 74

\begin{tabular}{lll}
\hline 6 & Channel capacity & $\mathbf{7 8}$
\end{tabular}

6.1 The general case . . . . . . . . . . . . . . . . . 78

6.1.1 The definition . . . . . . . . . . . . . . . . . . 78

6.1 .2 Simple calculations . . . . . . . . . . . . . . . . . 79

6.2 The classical capacity . . . . . . . . . . . . . . . . . . . . 81

6.2.1 Classical channels . . . . . . . . . . . . . . . . . . 81

6.2.2 Quantum channels ................. . . . 82

$6.2 .3 \quad$ Entanglement assisted capacity . . . . . . . . . . . . . . 83

6.2.4 Examples ...................... 83

6.3 The quantum capacity . . . . . . . . . . . . . . . . 87

6.3 .1 Alternative definitions . . . . . . . . . . . . . . . 87

6.3 .2 Upper bounds and achievable rates . . . . . . . . . . . . . . . 88

6.3 .3 Relations to entanglement measures . . . . . . . . . . . . . . 92 
\begin{tabular}{llr|}
\hline 7 & Multiple inputs & 94
\end{tabular}

7.1 The general scheme . . . . . . . . . . . . . . . . . . . . . 94

7.1 .1 Figures of merit . . . . . . . . . . . . . . . . . . 94

$7.1 .2 \quad$ Covariant operations . . . . . . . . . . . . . . . . . . . 95

$7.1 .3 \quad$ Group representations . . . . . . . . . . . . . . . . . . . 97

$7.1 .4 \quad$ Distillation of entanglement . . . . . . . . . . . . . . . . . . 99

7.2 Optimal devices $\ldots \ldots \ldots \ldots \ldots . \ldots \ldots$

$7.2 .1 \quad$ Optimal cloning . . . . . . . . . . . . . . . . . . . . 100

7.2 .2 Purification $\ldots \ldots \ldots \ldots$. . . . . . . . . . . . . . . . . . . 101

7.2 .3 Estimating pure states $\ldots \ldots \ldots \ldots$. . . . . . . . . . . 103

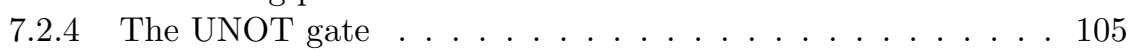

$7.3 \quad$ Asymptotic behaviour $\ldots \ldots \ldots \ldots$. . . . . . . . . . . . 105

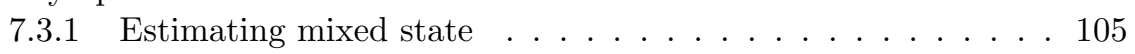

$7.3 .2 \quad$ Purification and cloning . . . . . . . . . . . . . . 107 


\section{Chapter 1}

\section{Introduction}

Quantum information and quantum computation have recently attracted a lot of interest. The promise of new technologies like safe cryptography and new "super computers", capable of handling otherwise untractable problems, has excited not only researchers from many different fields like physicists, mathematicians and computer scientists, but also a large public audience. On a practical level all these new visions are based on the ability to control the quantum states of (a small number of) micro systems individually and to use them for information transmission and processing. From a more fundamental point of view the crucial point is a reconsideration of the foundations of quantum mechanics in an information theoretical context. The purpose of this work is to follow the second path and to guide physicists into the theoretical foundations of quantum information and some of the most relevant topics of current research.

To this end the outline of this paper is as follows: The rest of this introduction is devoted to a rough and informal overview of the field, discussing some of its tasks and experimental realizations. Afterwards, in Chapter 2, we will consider the basic formalism which is necessary to present more detailed results. Typical keywords in this context are: systems, states, observables, correlations, entanglement and quantum channels. We then clarify these concepts (in particular entanglement and channels) with several examples in Chapter 3, and in Chapter 1 we discuss the most important tasks of quantum information in greater detail. The last three Chapters are devoted to a more quantitative analysis, where we make closer contact to current research: In Chapter 5 we will discuss how entanglement can be measured. The topic of Chapter 6 are channel capacities, i.e. we are looking at the amount of information which can maximally be transmitted over a noisy channel and in Chapter 7 we consider state estimation, optimal cloning and related tasks.

Quantum information is a rapidly developing field and the present work can of course reflect only a small part of it. An incomplete list of other general sources the reader should consult is: the books of Lo [111], Gruska [76], Nielsen and Chuang 122], Bouwmeester et. al. [23] and Alber et. al. [3], the lecture notes of Preskill [130] and the collection of references by Cabello 37 which particularly contains many references to other reviews.

\subsection{What is quantum information?}

Classical information is, roughly speaking, everything which can be transmitted from a sender to a receiver with "letters" from a "classical alphabet" e.g. the two digits " 0 " and " 1 " or any other finite set of symbols. In the context of classical information theory, it is completely irrelevant which type of physical system is used to perform the transmission. This abstract approach is successful because it is easy to transform information between different types of carriers like electric currents in a wire, laser pulses in an optical fiber, or symbols on a piece of paper without loss of data; and even if there are losses they are well understood and it is known how to deal with them. However, quantum information theory breaks with this point of view. It studies, loosely speaking, that kind of information ("quantum information") which is transmitted by micro particles from a preparation device (sender) to a measuring apparatus (receiver) in a quantum mechanical experiment - in other words the distinction between carriers of classical and quantum information becomes essential. This approach is justified by the observation that a lossless conversion of quantum information into classical information is in the above sense not possible. 
Therefore, quantum information is a new kind of information.

In order to explain why there is no way from quantum to classical information and back, let us discuss how such a conversion would look like. To convert quantum to classical information we need a device which takes quantum systems as input and produces classical information as output - this is nothing else than a measuring apparatus. The converse translation from classical to quantum information can be rephrased similarly as "parameter dependent preparation", i.e. the classical input to such a device is used to control the state (and possibly the type of system) in which the micro particles should be prepared. A combination of these two elements can be done in two ways. Let us first consider a device which goes from classical to quantum to classical information. This is a possible task and in fact technically realized already. A typical example is the transmission of classical information via an optical fiber. The information transmitted through the fiber is carried by micro particles (photons) and is therefore quantum information (in the sense of our preliminary definition). To send classical information we have to prepare first photons in a certain state send them through the channel and measure an appropriate observable at the output side. This is exactly the combination of a classical $\rightarrow$ quantum with a quantum $\rightarrow$ classical device just described.

The crucial point is now that the converse composition - performing the measurement $M$ first and the preparation $P$ afterwards (cf. Figure 1.1) - is more problematic. Such a process is called classical teleportation, if the particles produced by $P$ are "indistinguishable" from the input systems. We will show the impossibility of such a device via a hierarchy of other "impossible machines" which traces the problem back to the fundamental structure of quantum mechanics. This finally will prove our statement that quantum information is a new kind of information?.

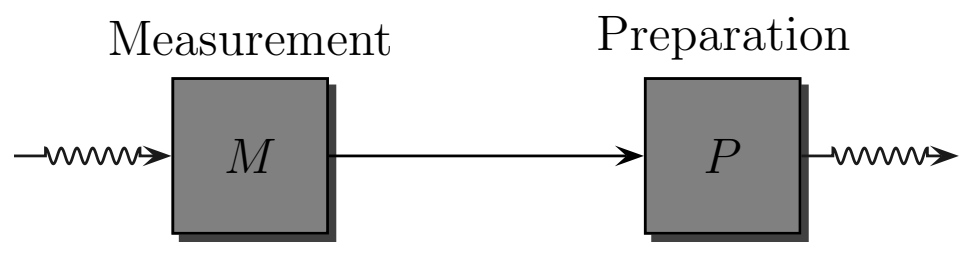

Figure 1.1: Schematic representation of classical teleportation. Here and in the following diagrams a curly arrow stands for quantum systems and a straight one for the flow of classical information.

To start with, we have to clarify the precise meaning of "indistinguishable" in this context. This has to be done in a statistical way, because the only possibility to compare quantum mechanical systems is in terms of statistical experiments. Hence we need an additional preparation device $P^{\prime}$ and an additional measuring apparatus $M^{\prime}$. Indistinguishable now means that it does not matter whether we perform $M^{\prime}$ measurements directly on $P^{\prime}$ outputs or whether we switch a teleportation device in between; cf. Figure 1.2. In both cases we should get the same distribution of measuring results for a large number of repetitions of the corresponding experiment. This requirement should hold for any preparation $P^{\prime}$ and any measurement $M^{\prime}$, but for fixed $M$ and $P$. The latter means that we are not allowed to use a priori knowledge about $P^{\prime}$ or $M^{\prime}$ to adopt the teleportation process (otherwise we can choose in the most extreme case always $P^{\prime}$ for $P$ and the whole discussion becomes meaningless).

The second impossible machine we have to consider is a quantum copying ma-

\footnotetext{
${ }^{1}$ The following chain of arguments is taken from 169, where it is presented in greater detail. This concerns in particular the construction of Bell's telephone from a joint measurement, which we have omitted here.
} 


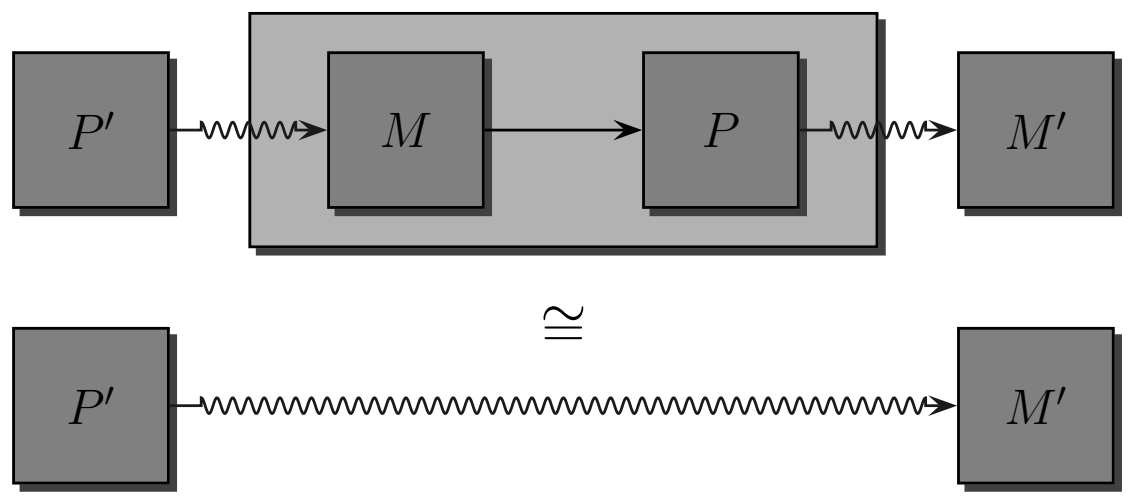

Figure 1.2: A teleportation process should not affect the results of a statistical experiment with quantum systems. A more precise explanation of the diagram is given in the text.

chine. This is a device $C$ which takes one quantum system $p$ as input and produces two systems $p_{1}, p_{2}$ of the same type as output. The limiting condition on $C$ is that $p_{1}$ and $p_{2}$ are indistinguishable from the input, where "indistinguishable" has to be understood in the same way as above: Any statistical experiment performed with one of the output particles (i.e. always with $p_{1}$ or always with $p_{2}$ ) yields the same result as applied directly to the input $p$. To get such a device from teleportation is easy: We just have to perform an $M$ measurement on $p$, make two copies of the classical data obtained, and run the preparation $P$ on each of them; cf. Figure 1.3 . Hence if teleportation is possible copying is possible as well.

According to the "no-cloning theorem" of Wootters and Zurek 173, however, a quantum copy machine does not exist and this basically concludes our proof. However we will give an easy argument for this theorem in terms of a third impossible machine - a joint measuring device $M_{A B}$ for two arbitrary observables $A$ and $B$. This is a measuring apparatus which produces each time it is invoked a pair $(a, b)$ of classical outputs, where $a$ is a possible output of $A$ and $b$ a possible output of $B$. The crucial requirement for $M_{A B}$ again is of statistical nature: The statistics of the $a$ outcomes is the same as for device $A$, and similarly for $B$. It is known from elementary quantum mechanics that many quantum observables are not jointly measurable in this way. The most famous examples are position and momentum or different components of angular momentum. Nevertheless a device $M_{A B}$ could be

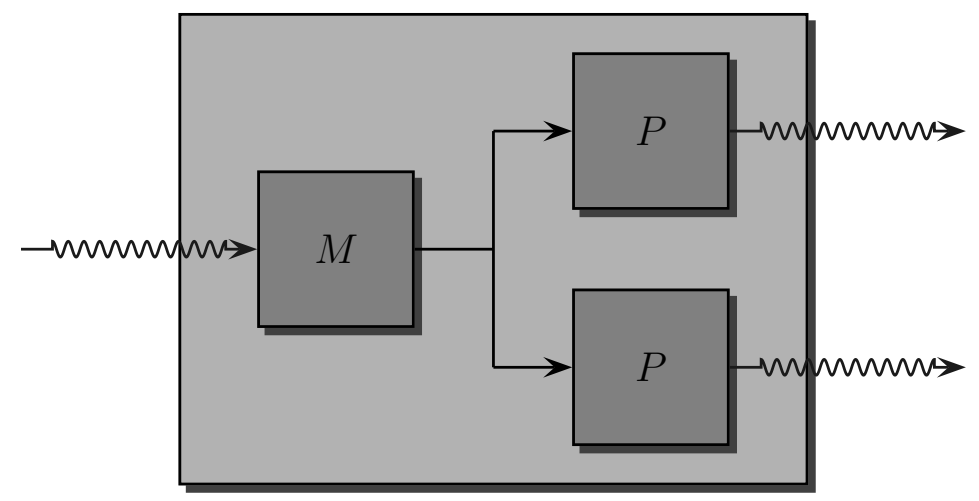

Figure 1.3: Constructing a quantum copying machine from a teleportation device. 


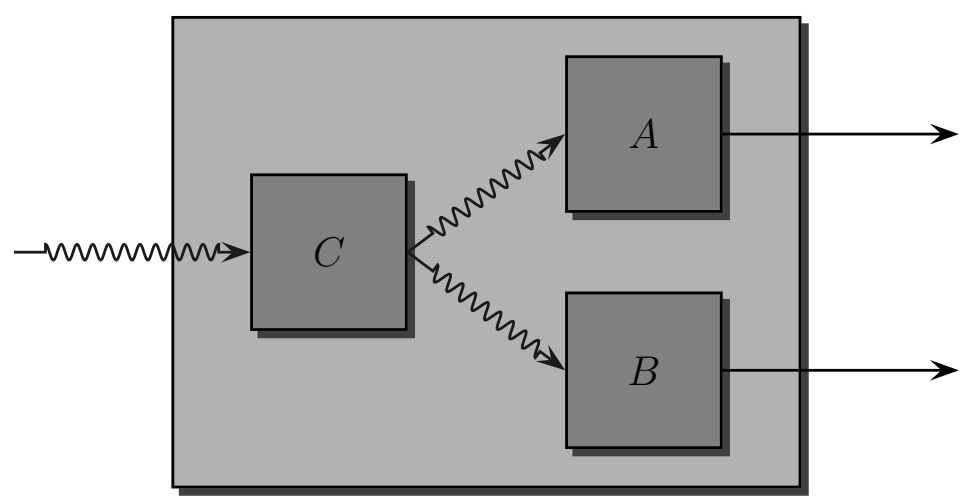

Figure 1.4: Constructing a joint measurement for the observables $A$ and $B$ from a quantum copying machine.

constructed for arbitrary $A$ and $B$ from a quantum copy machine $C$. We simply have to operate with $C$ on the input system $p$ producing two outputs $p_{1}$ and $p_{2}$ and to perform an $A$ measurement on $p_{1}$ and a $B$ measurement on $p_{2}$; cf. Figure 1.4. Since the outputs $p_{1}, p_{2}$ are, by assumption indistinguishable from the input $p$ the overall device constructed this way would give a joint measurement for $A$ and $B$. Hence a quantum copying machine cannot exist, as stated by the no-cloning theorem. This in turn implies that classical teleportation is impossible, and therefore we can not transform quantum information lossless into classical information and back. This concludes our chain of arguments.

\subsection{Tasks of quantum information}

So we have seen that quantum information is something new, but what can we do with it? There are three answers to this question which we want to present here. First of all let us remark that in fact all information in a modern data processing environment is carried by micro particles (e.g. electrons or photons). Hence quantum information comes automatically into play. Currently it is safe to ignore this and to use classical information theory to describe all relevant processes. If the size of the structures on a typical circuit decreases below a certain limit, however, this is no longer true and quantum information will become relevant.

This leads us to the second answer. Although it is far too early to say which concrete technologies will emerge from quantum information in the future, several interesting proposals show that devices based on quantum information can solve certain practical tasks much better than classical ones. The most well known and exciting one is, without a doubt, quantum computing. The basic idea is, roughly speaking, that a quantum computer can operate not only on one number per register but on superpositions of numbers. This possibility leads to an "exponential speedup" for some computations which makes problems feasible which are considered intractable by any classical algorithm. This is most impressively demonstrated by Shor's factoring algorithm 139, 140. A second example which is quite close to a concrete practical realization (i.e. outside the laboratory; see next Section) is quantum cryptography. The fact that it is impossible to perform a quantum mechanical measurement without disturbing the state of the measured system is used here for the secure transmission of a cryptographic key (i.e. each eavesdropping attempt can be detected with certainty). Together with a subsequent application of a classical encryption method known as the "one-time" pad this leads to a cryptographic scheme with provable security - in contrast to currently used public key systems whose security relies on possibly doubtful assumptions about (pseudo) ran- 
dom number generators and prime numbers. We will come back to both subjects quantum computing and quantum cryptography in Sections 4.5 and 4.6 .

The third answer to the above question is of more fundamental nature. The discussion of questions from information theory in the context of quantum mechanics leads to a deeper and in many cases more quantitative understanding of quantum theory. Maybe the most relevant example for this statement is the study of entanglement, i.e. non-classical correlations between quantum systems, which lead to

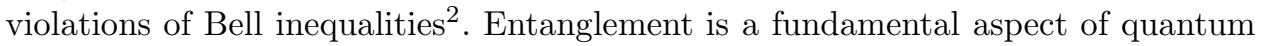
mechanics and demonstrates the differences between quantum and classical physics in the most drastical way - this can be seen from Bell-type experiments, like the one of Aspect et. al. [5], and the discussion about. Nevertheless, for a long time it was only considered as an exotic feature of the foundations of quantum mechanics which is not so relevant from a practical point of view. Since quantum information attained broader interest, however, this has changed completely. It has turned out that entanglement is an essential resource whenever classical information processing is outperformed by quantum devices. One of the most remarkable examples is the experimental realization of "entanglement enhanced" teleportation 24, 22]. We have argued in Section 1.1 that classical teleportation, i.e. transmission of quantum information through a classical information channel, is impossible. If sender and receiver share, however, an entangled pair of particles (which can be used as an additional resource) the impossible task becomes, most surprisingly, possible [11]! (We will discuss this fact in detail in Section 4.1.) The study of entanglement and in particular the question how it can be quantified is therefore a central topic within quantum information theory (cf. Chapter 5). Further examples for fields where quantum information has led to a deeper and in particular more quantitative insight include "capacities" of quantum information channels and "quantum cloning". A detailed discussion of these topics will be given in Chapter 6 and 2 . Finally let us remark that classical information theory benefits in a similar way from the synthesis with quantum mechanics. Beside the just mentioned channel capacities this concerns for example the theory of computational complexity which analyzes the scaling behavior of time and space consumed by an algorithm in dependence of the size of the input data. Quantum information challenges here in particular the fundamental Church-Turing hypotheses 45, 152 which claims that each computation can be simulated "efficiently" on a Turing machine; we come back to this topic in Section 4.5 .

\subsection{Experimental realizations}

Although this is a theoretical paper, it is of course necessary to say something about experimental realizations of the ideas of quantum information. Let us consider quantum computing first. Whatever way we go here, we need systems which can be prepared very precisely in few distinct states (i.e. we need "qubits"), which can be manipulated afterwards individually (we have to realize "quantum gates") and which can finally be measured with an appropriate observable (we have to "read out" the result).

One of the most far developed approaches to quantum computing is the ion trap technique (see Section 4.3 and 5.3 in [23] and Section 7.6 of 122 for an overview and further references). A "quantum register" is realized here by a string of ions kept by electromagnetic fields in high vacuum inside a Paul trap, and two longliving states of each ion are chosen to represent "0" and "1". A single ion can be manipulated by laser beams and this allows the implementation of all "one-qubit gates". To get two-qubit gates as well (for a quantum computer we need at least one two qubit gate together with all one-qubit operations; cf. Section 4.5) the collective

\footnotetext{
${ }^{2}$ This is only a very rough characterization. A more precise one will be given in Section 2.2 .
} 
motional state of the ions has to be used. A "program" on an ion trap quantum computer starts now with a preparation of the register in an initial state - usually the ground state of the ions. This is done by optical pumping and laser cooling (which is in fact one of the most difficult parts of the whole procedure, in particular if many ions are involved). Then the "network" of quantum gates is applied, in terms of a (complicated) sequence of laser pulses. The readout finally is done by laser beams which illuminate the ions subsequently. The beams are tuned to a fast transition which affects only one of the qubit states and the fluorescent light is detected. Concrete implementations (see e.g. [118, 102]) are currently restricted to two qubits, however there is some hope that we will be able to control up to 10 or 12 qubits in the not too distant future.

A second quite successful technique is NMR quantum computing (see Section 5.4 of 23] and Section 7.7 of 122] together with the references therein for details). NMR stands for "nuclear magnetic resonance" and it is the study of transitions between Zeeman levels of an atomic nucleus in a magnetic field. The qubits are in this case different spin states of the nuclei in an appropriate molecule and quantum gates are realized by high frequency oscillating magnetic fields in pulses of controlled duration. In contrast to ion traps however we do not use one molecule but a whole cup of liquid containing some $10^{20}$ of them. This causes a number of problems, concerning in particular the preparation of an initial state, fluctuations in the free time evolution of the molecules and the readout. There are several ways to overcome these difficulties and we refer the reader again to [23] and [122] for details. Concrete implementations of NMR quantum computers are capable to use up to five qubits [113. Other realizations include the implementation of several known quantum algorithms on two and three qubits; see e.g. 44, 96, 109].

The fundamental problem of the two methods for quantum computation discussed so far, is their lack of scalability. It is realistic to assume that NMR and ion-trap quantum computer with up to tens of qubits will exist somewhen in the future but not with thousands of qubits which are necessary for "real world" applications. There are, however, many other alternative proposals available and some of them might be capable to avoid this problem. The following is a small (not at all exhaustive) list: atoms in optical lattices [28], semiconductor nanostructures such as quantum dots (there are many works in this area, some recent are 149, 30, 21, 29) and arrays of Josephson junctions [112].

A second circle of experiments we want to mention here is grouped around quantum communication and quantum cryptography (for a more detailed overview let us refer to [163] and 696]). Realizations of quantum cryptography are fairly far developed and it is currently possible to span up to $50 \mathrm{~km}$ with optical fibers (e.g. [93]). Potentially greater distances can be bridged by "free space cryptography" where the quantum information is transmitted through the air (e.g [34]). With this technology satellites can be used as some sort of "relays", thus enabling quantum key distribution over arbitrary distances. In the meantime there are quite a lot of successful implementations. For a detailed discussion we will refer the reader to the review of Gisin et. al. [69] and the references therein. Other experiments concern the usage of entanglement in quantum communication. The creation and detection of entangled photons is here a fundamental building block. Nowadays this is no problem and the most famous experiment in this context is the one of Aspect et. al. [5], where the maximal violation of Bell inequalities was demonstrated with polarization correlated photons. Another spectacular experiment is the creation of entangled photons over a distance of $10 \mathrm{~km}$ using standard telecommunication optical fibers by the Geneva group [151. Among the most exciting applications of entanglement is the realization of entanglement based quantum key distribution [95], the first successful "teleportation" of a photon [24, 22] and the implementation of "dense coding" 115; cf. Section 4.1. 


\section{Chapter 2}

\section{Basic concepts}

After we have got a first, rough impression of the basic ideas and most relevant subjects of quantum information theory, let us start with a more detailed presentation. First we have to introduce the fundamental notions of the theory and their mathematical description. Fortunately, much of the material we should have to present here, like Hilbert spaces, tensor products and density matrices, is known already from quantum mechanics and we can focus our discussion to those concepts which are less familiar like POV measures, completely positive maps and entangled states.

\subsection{Systems, States and Effects}

As classical probability theory quantum mechanics is a statistical theory. Hence its predictions are of probabilistic nature and can only be tested if the same experiment is repeated very often and the relative frequencies of the outcomes are calculated. In more operational terms this means: the experiment has to be repeated according to the same procedure as it can be set out in a detailed laboratory manual. If we consider a somewhat idealized model of such a statistical experiment we get in fact two different types of procedures: first preparation procedures which prepare a certain kind of physical system in a distinguished state and second registration procedures measuring a particular observable.

A mathematical description of such a setup basically consists of two sets $\mathcal{S}$ and $\mathcal{E}$ and a map $\mathcal{S} \times \mathcal{E} \ni(\rho, A) \rightarrow \rho(A) \in[0,1]$. The elements of $\mathcal{S}$ describe the states, i.e. preparations, while the $A \in \mathcal{E}$ represent all yes/no measurements (effects) which can be performed on the system. The probability (i.e. the relative frequency for a large number of repetitions) to get the result "yes", if we are measuring the effect $A$ on a system prepared in the state $\rho$, is given by $\rho(A)$. This is a very general scheme applicable not only to quantum mechanics but also to a very broad class of statistical models, containing in particular classical probability. In order to make use of it we have to specify of course the precise structure of the sets $\mathcal{S}$ and $\mathcal{E}$ and the map $\rho(A)$ for the types of systems we want to discuss.

2.1.1. Operator algebras. — Throughout this paper we will encounter three different kinds of systems: quantum and classical systems and hybrid systems which are half classical, half quantum (cf. Subsection 2.2.2). In this subsection we will describe a general way to define states and effects which is applicable to all three cases and which therefore provides a handy way to discuss all three cases simultaneously (this will become most useful in Section 2.2 and 2.3).

The scheme we are going to discuss is based on an algebra $\mathcal{A}$ of bounded operators acting on a Hilbert space $\mathcal{H}$. More precisely $\mathcal{A}$ is a (closed) linear subspace of $\mathcal{B}(\mathcal{H})$, the algebra of bounded operates on $\mathcal{H}$, which contains the identity $(\mathbb{I} \in \mathcal{A})$ and is closed under products $(A, B \in \mathcal{A} \Rightarrow A B \in \mathcal{A})$ and adjoints $(A \in \mathcal{A}$ $\left.\Rightarrow A^{*} \in \mathcal{A}\right)$. For simplicity we will refer to each such $\mathcal{A}$ as an observable algebra. The key observation is now that each type of system we will study in the following can be completely characterized by its observable algebra $\mathcal{A}$, i.e. once $\mathcal{A}$ is known there is a systematic way to derive the sets $\mathcal{S}$ and $\mathcal{E}$ and the map $(\rho, A) \mapsto \rho(A)$ from it. We frequently make use of this fact by referring to systems in terms of their observable algebra $\mathcal{A}$, or even by identifying them with their algebra and saying that $\mathcal{A}$ is the system.

Although $\mathcal{A}$ and $\mathcal{H}$ can be infinite dimensional in general, we will consider only 
finite dimensional Hilbert spaces, as long as nothing else is explicitly stated. Since most research in quantum information is done up to now for finite dimensional systems (the only exception in this work is the discussion of Gaussian systems in Section 3.3 this is not a too severe loss of generality. Hence we can choose $\mathcal{H}=\mathbb{C}^{d}$ and $\mathcal{B}(\mathcal{H})$ is just the algebra of complex $d \times d$ matrices. Since $\mathcal{A}$ is a subalgebra of $\mathcal{B}(\mathcal{H})$ it operates naturally on $\mathcal{H}$ and it inherits from $\mathcal{B}(\mathcal{H})$ the operator norm $\|A\|=\sup _{\|\psi\|=1}\|A \psi\|$ and the operator ordering $A \geq B \Leftrightarrow\langle\psi, A \psi\rangle \geq\langle\psi, B \psi\rangle$ $\forall \psi \in \mathcal{H}$. Now we can define:

$$
\mathcal{S}(\mathcal{A})=\left\{\rho \in \mathcal{A}^{*} \mid \rho \geq 0, \rho(\mathbb{I})=1\right\}
$$

where $\mathcal{A}^{*}$ denotes the dual space of $\mathcal{A}$, i.e. the set of all linear functionals on $\mathcal{A}$, and $\rho \geq 0$ means $\rho(A) \geq 0 \forall A \geq 0$. Elements of $\mathcal{S}(\mathcal{A})$ describe the states of the system in question while effects are given by

$$
\mathcal{E}(\mathcal{A})=\{A \in \mathcal{A} \mid A \geq 0, A \leq \mathbb{I}\}
$$

The probability to measure the effect $A$ in the state $\rho$ is $\rho(A)$. More generally we can look at $\rho(A)$ for an arbitrary $A$ as the expectation value of $A$ in the state $\rho$. Hence the idea behind Equation (2.1) is to define states in terms of their expectation value functionals.

Both spaces are convex, i.e. $\rho, \sigma \in \mathcal{S}(\mathcal{A})$ and $0 \leq \lambda \leq 1$ implies $\lambda \rho+(1-\lambda) \sigma \in$ $\mathcal{S}(\mathcal{A})$ and similarly for $\mathcal{E}(\mathcal{A})$. The extremal points of $\mathcal{S}(\mathcal{A})$ respectively $\mathcal{E}(\mathcal{A})$, i.e. those elements which do not admit a proper convex decomposition $(x=\lambda y+(1-\lambda) z \Rightarrow$ $\lambda=1$ or $\lambda=0$ or $y=z=x)$, play a distinguished role: the extremal points of $\mathcal{S}(\mathcal{A})$ are pure states and those of $\mathcal{E}(\mathcal{A})$ are the propositions of the system in question. The latter represent those effects which register a property with certainty in contrast to non-extremal effects which admit some "fuzziness". As a simple example for the latter consider a detector which registers particles not with certainty but only with a probability which is smaller than one.

Finally let us note that the complete discussion of this section can be generalized easily to infinite dimensional systems, if we replace $\mathcal{H}=\mathbb{C}^{d}$ by an infinite dimensional Hilbert space (e.g. $\mathcal{H}=\mathrm{L}^{2}(\mathbb{R})$ ). This would require however more material about $\mathrm{C}^{*}$ algebras and measure theory than we want to use in this paper.

2.1.2. Quantum mechanics. - For quantum mechanics we have

$$
\mathcal{A}=\mathcal{B}(\mathcal{H})
$$

where we have chosen again $\mathcal{H}=\mathbb{C}^{d}$. The corresponding systems are called $d$-level systems or qubits if $d=2$ holds. To avoid clumsy notations we frequently write $\mathcal{S}(\mathcal{H})$ and $\mathcal{E}(\mathcal{H})$ instead of $\mathcal{S}[\mathcal{B}(\mathcal{H})]$ and $\mathcal{E}[\mathcal{B}(\mathcal{H})]$. From Equation (2.2) we immediately see that an operator $A \in \mathcal{B}(\mathcal{H})$ is an effect iff it is positive and bounded from above by $\mathbb{I}$. An element $P \in \mathcal{E}(\mathcal{H})$ is a propositions iff $P$ is a projection operator $\left(P^{2}=P\right)$.

States are described in quantum mechanics usually by density matrices, i.e. positive and normalized trace class $\prod^{\top}$ operators. To make contact to the general definition in Equation (2.1) note first that $\mathcal{B}(\mathcal{H})$ is a Hilbert space with the HilbertSchmidt scalar product $\langle A, B\rangle=\operatorname{tr}\left(A^{*} B\right)$. Hence each linear functional $\rho \in \mathcal{B}(\mathcal{H})^{*}$ can be expressed in terms of a (trace class) operator $\widetilde{\rho}$ by ${ }^{2} A \mapsto \rho(A)=\operatorname{tr}(\widetilde{\rho} A)$. It is

\footnotetext{
${ }^{1}$ On a finite dimensional Hilbert space this attribute is of course redundant, since each operator is of trace class in this case. Nevertheless we will frequently use this terminology, due to greater consistency with the infinite dimensional case.

2 If we consider infinite dimensional systems this is not true. In this case the dual space of the observable algebra is much larger and Equation (2.1) leads to states which are not necessarily given by trace class operators. Such "singular states" play an important role in theories which admit an infinite number of degrees of freedom like quantum statistics and quantym field theory; cf. 25, 26]. For applications of singular states within quantum information see [97].
} 
obvious that each $\widetilde{\rho}$ defines a unique functional $\rho$. If we start on the other hand with $\rho$ we can recover the matrix elements of $\widetilde{\rho}$ from $\rho$ by $\widetilde{\rho}_{k j}=\operatorname{tr}(\widetilde{\rho}|j\rangle\langle k|)=\rho(|j\rangle\langle k|)$, where $|j\rangle\langle k|$ denotes the canonical basis of $\mathcal{B}(\mathcal{H})$ (i.e. $|j\rangle\left\langle\left. k\right|_{a b}=\delta_{j a} \delta_{k b}\right.$ ). More generally we get for $\psi, \phi \in \mathcal{H}$ the relation $\langle\phi, \widetilde{\rho} \psi\rangle=\rho(|\psi\rangle\langle\phi|)$, where $|\psi\rangle\langle\phi|$ now denotes the rank one operator which maps $\eta \in \mathcal{H}$ to $\langle\phi, \eta\rangle \psi$. In the following we drop the $\sim$ and use the same symbol for the operator and the functional whenever confusion can be avoided. Due to the same abuse of language we will interpret elements of $\mathcal{B}(\mathcal{H})^{*}$ frequently as (trace class) operators instead of linear functionals (and write $\operatorname{tr}(\rho A)$ instead of $\rho(A)$ ). However we do not identify $\mathcal{B}(\mathcal{H})^{*}$ with $\mathcal{B}(\mathcal{H})$ in general, because the two different notations help to keep track of the distinction between spaces of states and spaces of observables. In addition we equip $\mathcal{B}^{*}(\mathcal{H})$ with the trace-norm $\|\rho\|_{1}=\operatorname{tr}|\rho|$ instead of the operator norm.

Positivity of the functional $\rho$ implies positivity of the operator $\rho$ due to $0 \leq \rho(|\psi\rangle\langle\psi|)=\langle\psi, \rho \psi\rangle$ and the same holds for normalization: $1=\rho(\mathbb{I})=\operatorname{tr}(\rho)$. Hence we can identify the state space from Equation (2.1) with the set of density matrices, as expected for quantum mechanics. Pure states of a quantum system are the one dimensional projectors. As usual we will frequently identify the density matrix $|\psi\rangle\langle\psi|$ with the wave function $\psi$ and call the latter in abuse of language a state.

To get a useful parameterization of the state space consider again the HilbertSchmidt scalar product $\langle\rho, \sigma\rangle=\operatorname{tr}\left(\rho^{*} \sigma\right)$, but now on $\mathcal{B}^{*}(\mathcal{H})$. The space of trace free matrices in $\mathcal{B}^{*}(\mathcal{H})$ (alternatively the functionals with $\rho(\mathbb{I})=0$ ) is the corresponding orthocomplement $\mathbb{I}^{\perp}$ of the unit operator. If we choose a basis $\sigma_{1}, \ldots, \sigma_{d^{2}-1}$ with $\left\langle\sigma_{j}, \sigma_{k}\right\rangle=2 \delta_{j k}$ in $\mathbb{I}^{\perp}$ we can write each selfajoint (trace class) operator $\rho$ with $\operatorname{tr}(\rho)=1$ as

$$
\rho=\frac{\mathbb{I}}{d}+\frac{1}{2} \sum_{j=1}^{d^{2}-1} x_{j} \sigma_{j}=: \frac{\mathbb{I}}{d}+\frac{1}{2} \vec{x} \cdot \vec{\sigma}, \text { with } \vec{x} \in \mathbb{R}^{d^{2}-1} .
$$

If $d=2$ or $d=3$ holds, it is most natural to choose the Pauli matrices respectively the Gell-Mann matrices (cf. e.g. Sect. 13.4 of [48]) for the $\sigma_{j}$. In the qubit case it is easy to see that $\rho \geq 0$ holds iff $|\vec{x}| \leq 1$. Hence the state space $\mathcal{S}\left(\mathbb{C}^{2}\right)$ coincides with the Bloch ball $\left\{\vec{x} \in \mathbb{R}^{3}|| \vec{x} \mid \leq 1\right\}$, and the set of pure states with its boundary, the Bloch sphere $\left\{\vec{x} \in \mathbb{R}^{3}|| \vec{x} \mid=1\right\}$. This shows in a very geometric way that the pure states are the extremal points of the convex set $\mathcal{S}(\mathcal{H})$. If $\rho$ is more generally a pure state of a $d$-level system we get

$$
1=\operatorname{tr}\left(\rho^{2}\right)=\frac{1}{d}+\frac{1}{2}|\vec{x}|^{2} \Rightarrow|\vec{x}|=\sqrt{2(1-1 / d)} .
$$

This implies that all states are contained in the ball with radius $2^{1 / 2}(1-1 / d)^{1 / 2}$, however not all operators in this set are positive. A simple example is $d^{-1} \mathbb{1} \pm 2^{1 / 2}(1-$ $1 / d)^{1 / 2} \sigma_{j}$, which is positive only if $d=2$ holds.

2.1.3. Classical probability. — Since the difference between classical and quantum systems is an important issue in this work let us reformulate classical probability theory according to the general scheme from Subsection 2.1.1. The restriction to finite dimensional observable algebras leads now to the assumption that all systems we are considering admit a finite set $X$ of elementary events. Typical examples are: throwing a dice $X=\{1, \ldots, 6\}$, tossing a coin $X=\{$ "head", "number" $\}$ or classical bits $X=\{0,1\}$. To simplify the notations we write (as in quantum mechanics) $\mathcal{S}(X)$ and $\mathcal{E}(X)$ for the spaces of states and effects.

The observable algebra $\mathcal{A}$ of such a system is the space

$$
\mathcal{A}=\mathcal{C}(X)=\{f: X \rightarrow \mathbb{C}\}
$$


of complex valued functions on $X$. To interpret this as an operator algebra acting on a Hilbert space $\mathcal{H}$ (as indicated in Subsection 2.1.1) choose an arbitrary but fixed orthonormal basis $|x\rangle, x \in X$ in $\mathcal{H}$ and identify the function $f \in \mathcal{C}(X)$ with the operator $f=\sum_{x} f_{x}|x\rangle\langle x| \in \mathcal{B}(\mathcal{H})$ (we use the same symbol for the function and the operator, provided confusion can be avoided). Most frequently we have $X=\{1, \ldots, d\}$ and we can choose $\mathcal{H}=\mathbb{C}^{d}$ and the canonical basis for $|x\rangle$. Hence $\mathcal{C}(X)$ becomes the algebra of diagonal $d \times d$ matrices. Using Equation (2.2) we immediately see that $f \in \mathcal{C}(X)$ is an effect iff $0 \leq f_{x} \leq 1, \forall x \in X$. Physically we can interpret $f_{x}$ as the probability that the effect $f$ registers the elementary event $x$. This makes the distinction between propositions and "fuzzy" effects very transparent: $P \in \mathcal{E}(X)$ is a proposition iff we have either $P_{x}=1$ or $P_{x}=0$ for all $x \in X$. Hence the propositions $P \in \mathcal{C}(X)$ are in one to one correspondence with the subsets $\omega_{P}=\left\{x \in X \mid P_{x}=1\right\} \subset X$ which in turn describe the events of the system. Hence $P$ registers the event $\omega_{P}$ with certainty, while a fuzzy effect $f<P$ does this only with a probability less then one.

Since $\mathcal{C}(X)$ is finite dimensional and admits the distinguished basis $|x\rangle\langle x|, x \in X$ it is naturally isomorphic to its dual $\mathcal{C}^{*}(X)$. More precisely: each linear functional $\rho \in \mathcal{C}^{*}(X)$ defines and is uniquely defined by the function $x \mapsto \rho_{x}=\rho(|x\rangle\langle x|)$ and we have $\rho(f)=\sum_{x} f_{x} \rho_{x}$. As in the quantum case we will identify the function $\rho$ with the linear functional and use the same symbol for both, although we keep the notation $\mathcal{C}^{*}(X)$ to indicate that we are talking about states rather than observables.

Positivity of $\rho \in \mathcal{C}^{*}(X)$ is given by $\rho_{x} \geq 0$ for all $x$ and normalization leads to $1=\rho(\mathbb{I})=\rho\left(\sum_{x}|x\rangle\langle x|\right)=\sum_{x} \rho_{x}$. Hence to be a state $\rho \in \mathcal{C}^{*}(X)$ must be a probability distribution on $X$ and $\rho_{j}$ is the probability that the elementary event $x$ occurs during statistical experiments with systems in the state $\rho$. More generally $\rho(f)=\sum_{j} \rho_{j} f_{j}$ is the probability to measure the effect $f$ on systems in the state $\rho$. If $P$ is in particular a proposition, $\rho(P)$ gives the probability for the event $\omega_{P}$. The pure states of the system are the Dirac measures $\delta_{x}, x \in X$; with $\delta_{x}(|y\rangle\langle y|)=\delta_{x y}$. Hence each $\rho \in \mathcal{S}(X)$ can be decomposed in a unique way into a convex linear combination of pure states.

2.1.4. Observables. - Up to now we have discussed only effects, i.e. yes/no experiments. In this subsection we will have a first short look at more general observables. We will come back to this topic in Section 3.2.4 after we have introduced channels. We can think of an observable $E$ taking its values in a finite set $X$ as a map which associates to each possible outcome $x \in X$ the effect $E_{x} \in \mathcal{E}(\mathcal{A}$ ) (if $\mathcal{A}$ is the observable algebra of the system in question) which is true if $x$ is measured and false otherwise. If the measurement is performed on systems in the state $\rho$ we get for each $x \in X$ the probability $p_{x}=\rho\left(E_{x}\right)$ to measure $x$. Hence the family of the $p_{x}$ should be a probability distribution on $X$, and this implies that $E$ should be a POV measure on $X$.

DeFinition 2.1.1 Consider an observable algebra $\mathcal{A} \subset \mathcal{B}(\mathcal{H})$ and a finit f $^{3}$ set $X$. $A$ family $E=\left(E_{x}\right)_{x \in X}$ of effects in $\mathcal{A}$ (i.e. $0 \leq E_{x} \leq \mathbb{I}$ ) is called a positive operator valued measure (POV measure) on $X$ if $\sum_{x \in X} E_{x}=\mathbb{I}$ holds. If all $E_{x}$ are projections, $E$ is called projection valued measure ( $P V$ measure).

From basic quantum mechanics we know that observables are described by self adjoint operators on a Hilbert space $\mathcal{H}$. But, how does this point of view fit into the previous definition? The answer is given by the spectral theorem (Thm. VIII.6 [134): Each selfadjoint operator $A$ on a finite dimensional Hilbert space $\mathcal{H}$ has the form $A=\sum_{\lambda \in \sigma(A)} \lambda P_{\lambda}$ where $\sigma(A)$ denotes the spectrum of $A$, i.e. the set of

\footnotetext{
${ }^{3}$ This is if course an artifical restriction and in many situations not justified (cf. in particular the discussion of quantum state estimation in Section 42 and Chapter 0 ). However, it helps us to avoid measure theoretical subtleties; cf. Holevo's book [79] for a more general discussion.
} 
eigenvalues and $P_{\lambda}$ denotes the projection onto the corresponding eigenspace. Hence there is a unique PV measure $P=\left(P_{\lambda}\right)_{\lambda \in \sigma(A)}$ associated to $A$ which is called the spectral measure of $A$. It is uniquely characterized by the property that the expectation value $\sum_{\lambda} \lambda \rho\left(P_{\lambda}\right)$ of $P$ in the state $\rho$ is given for any state $\rho$ by $\rho(A)=\operatorname{tr}(\rho A)$; as it is well known from quantum mechanics. Hence the traditional way to define observables within quantum mechanics perfectly fits into the scheme just outlined, however it only covers the projection valued case and therefore admits no fuzziness. For this reason POV measures are sometimes called generalized observables.

Finally note that the eigenprojections $P_{\lambda}$ of $A$ are elements of an observable algebra $\mathcal{A}$ iff $A \in \mathcal{A}$. This shows two things: First of all we can consider selfadjoint elements of any ${ }^{*}$-subalgebra $\mathcal{A}$ of $\mathcal{B}(\mathcal{H})$ as observables of $\mathcal{A}$-systems, and this is precisely the reason why we have called $\mathcal{A}$ observable algebra. Secondly we see why it is essential that $\mathcal{A}$ is really a subalgebra of $\mathcal{B}(\mathcal{H})$ : if it is only a linear subspace of $\mathcal{B}(\mathcal{H})$ the relation $A \in \mathcal{A}$ does not imply $P_{\lambda} \in \mathcal{A}$.

\subsection{Composite systems and entangled states}

composite systems occur in many places in quantum information theory. A typical example is a register of a quantum computer, which can be regarded as a system consisting of $N$ qubits (if $N$ is the length of the register). The crucial point is that this opens the possibility for correlations and entanglement between subsystems. In particular entanglement is of great importance, because it is a central resource in many applications of quantum information theory like entanglement enhanced teleportation or quantum computing - we already discussed this in Section 1.2 of the introduction. To explain entanglement in greater detail and to introduce some necessary formalism we have to complement the scheme developed in the last section by a procedure which allows us to construct states and observables of the composite system from its subsystems. In quantum mechanics this is done of course in terms of tensor products, and we will review in the following some of the most relevant material.

2.2.1. Tensor products. - Consider two (finite dimensional) Hilbert spaces $\mathcal{H}$ and $\mathcal{K}$. To each pair of vectors $\psi_{1} \in \mathcal{H}, \psi_{2} \in \mathcal{K}$ we can associate a bilinear form $\psi_{1} \otimes \psi_{2}$ called the tensor product of $\psi_{1}$ and $\psi_{2}$ by $\psi_{1} \otimes \psi_{2}\left(\phi_{1}, \phi_{2}\right)=\left\langle\psi_{1}, \phi_{1}\right\rangle\left\langle\psi_{2}, \phi_{2}\right\rangle$. For two product vectors $\psi_{1} \otimes \psi_{2}$ and $\eta_{1} \otimes \eta_{2}$ their scalar product is defined by $\left\langle\psi_{1} \otimes \psi_{2}, \eta_{1} \otimes \eta_{2}\right\rangle=\left\langle\psi_{1}, \eta_{1}\right\rangle\left\langle\psi_{2}, \eta_{2}\right\rangle$ and it can be shown that this definition extends in a unique way to the span of all $\psi_{1} \otimes \psi_{2}$ which therefore defines the tensor product $\mathcal{H} \otimes \mathcal{K}$. If we have more than two Hilbert spaces $\mathcal{H}_{j}, j=1, \ldots, N$ their tensor product $\mathcal{H}_{1} \otimes \cdots \otimes \mathcal{H}_{N}$ can be defined similarly.

The tensor product $A_{1} \otimes A_{2}$ of two bounded operators $A_{1} \in \mathcal{B}(\mathcal{H}), A_{2} \in \mathcal{B}(\mathcal{K})$ is defined first for product vectors $\psi_{1} \otimes \psi_{2} \in \mathcal{H} \otimes \mathcal{K}$ by $A_{1} \otimes A_{2}\left(\psi_{1} \otimes \psi_{2}\right)=$ $\left(A_{1} \psi_{1}\right) \otimes\left(A_{2} \psi_{2}\right)$ and then extended by linearity. The space $\mathcal{B}(\mathcal{H} \otimes \mathcal{K})$ coincides with the span of all $A_{1} \otimes A_{2}$. If $\rho \in \mathcal{B}(\mathcal{H} \otimes \mathcal{K})$ is not of product form (and of trace class for infinite dimensional $\mathcal{H}$ and $\mathcal{K}$ ) there is nevertheless a way to define "restrictions" to $\mathcal{H}$ respectively $\mathcal{K}$ called the partial trace of $\rho$. It is defined by the equation

$$
\operatorname{tr}\left[\operatorname{tr}_{\mathcal{K}}(\rho) A\right]=\operatorname{tr}(\rho A \otimes \mathbb{I}) \quad \forall A \in \mathcal{B}(\mathcal{H})
$$

where the trace on the left hand side is over $\mathcal{H}$ and on the right hand side over $\mathcal{H} \otimes \mathcal{K}$.

If two orthonormal bases $\phi_{1}, \ldots, \phi_{n}$ and $\psi_{1}, \ldots, \psi_{m}$ are given in $\mathcal{H}$ respectively $\mathcal{K}$ we can consider the product basis $\phi_{1} \otimes \psi_{1}, \ldots, \phi_{n} \otimes \psi_{m}$ in $\mathcal{H} \otimes \mathcal{K}$, and we can expand each $\Psi \in \mathcal{H} \otimes \mathcal{K}$ as $\Psi=\sum_{j k} \Psi_{j k} \phi_{j} \otimes \psi_{k}$ with $\Psi_{j k}=\left\langle\phi_{j} \otimes \psi_{k}, \Psi\right\rangle$. This procedure works for an arbitrary number of tensor factors. However, if we have 
exactly a twofold tensor product, there is a more economic way to expand $\Psi$, called Schmidt decomposition in which only diagonal terms of the form $\phi_{j} \otimes \psi_{j}$ appear.

Proposition 2.2.1 For each element $\Psi$ of the twofold tensor product $\mathcal{H} \otimes \mathcal{K}$ there are orthonormal systems $\phi_{j}, j=1, \ldots, n$ and $\psi_{k}, k=1, \ldots, n$ (not necessarily bases, i.e. $n$ can be smaller than $\operatorname{dim} \mathcal{H}$ and $\operatorname{dim} \mathcal{K})$ of $\mathcal{H}$ and $\mathcal{K}$ respectively such that $\Psi=\sum_{j} \sqrt{\lambda}_{j} \phi_{j} \otimes \psi_{j}$ holds. The $\phi_{j}$ and $\psi_{j}$ are uniquely determined by $\Psi$. The expansion is called Schmidt decomposition and the numbers $\sqrt{\lambda}_{j}$ are the Schmidt coefficients.

Proof. Consider the partial trace $\rho_{1}=\operatorname{tr}_{\mathcal{K}}(|\Psi\rangle\langle\Psi|)$ of the one dimensional projector $|\Psi\rangle\langle\Psi|$ associated to $\Psi$. It can be decomposed in terms of its eigenvectors $\phi_{n}$ and we get $\operatorname{tr}_{\mathcal{K}}(|\Psi\rangle\langle\Psi|)=\rho_{1}=\sum_{n} \lambda_{n}\left|\phi_{n}\right\rangle\left\langle\phi_{n}\right|$. Now we can choose an orthonormal basis $\psi_{k}^{\prime}, k=1, \ldots, m$ in $\mathcal{K}$ and expand $\Psi$ with respect to $\phi_{j} \otimes \psi_{k}^{\prime}$. Carrying out the $k$ summation we get a family of vectors $\psi_{j}^{\prime \prime}=\sum_{k}\left\langle\Psi, \phi_{j} \otimes \psi_{k}^{\prime}\right\rangle \psi_{k}^{\prime}$ with the property $\Psi=\sum_{j} \phi_{j} \otimes \psi_{j}^{\prime \prime}$. Now we can calculate the partial trace and get for any $A \in \mathcal{B}\left(\mathcal{H}_{1}\right)$ :

$$
\sum_{j} \lambda_{j}\left\langle\phi_{j}, A \phi_{j}\right\rangle=\operatorname{tr}\left(\rho_{1} A\right)=\langle\Psi,(A \otimes \mathbb{I}) \Psi\rangle=\sum_{j, k}\left\langle\phi_{j}, A \phi_{k}\right\rangle\left\langle\psi_{j}^{\prime \prime}, \psi_{k}^{\prime \prime}\right\rangle .
$$

Since $A$ is arbitrary we can compare the left and right hand side of this equation term by term and we get $\left\langle\psi_{j}^{\prime \prime}, \psi_{k}^{\prime \prime}\right\rangle=\delta_{j k} \lambda_{j}$. Hence $\psi_{j}=\lambda_{j}^{-1 / 2} \psi_{j}^{\prime \prime}$ is the desired orthonormal system.

As an immediate application of this result we can show that each mixed state $\rho \in \mathcal{B}^{*}(\mathcal{H})$ (of the quantum system $\mathcal{B}(\mathcal{H})$ ) can be regarded as a pure state on a larger Hilbert space $\mathcal{H} \otimes \mathcal{H}^{\prime}$. We just have to consider the eigenvalue expansion $\rho=$ $\sum_{j} \lambda_{j}\left|\phi_{j}\right\rangle\left\langle\phi_{j}\right|$ of $\rho$ and to choose an arbitrary orthonormal system $\psi_{j}, j=1, \ldots n$ in $\mathcal{H}^{\prime}$. Using Proposition 2.2.1 we get

Corollary 2.2.2 Each state $\rho \in \mathcal{B}^{*}(\mathcal{H})$ can be extended to a pure state $\Psi$ on a larger system with Hilbert space $\mathcal{H} \otimes \mathcal{H}^{\prime}$ such that $\operatorname{tr}_{\mathcal{H}^{\prime}}|\Psi\rangle\langle\Psi|=\rho$ holds.

2.2.2. Compound and hybrid systems. - To discuss the composition of two arbitrary (i.e. classical or quantum) systems it is very convenient to use the scheme developed in Subsection 2.1.1 and to talk about the two subsystems in terms of their observable algebras $\mathcal{A} \subset \mathcal{B}(\mathcal{H})$ and $\mathcal{B} \subset \mathcal{B}(\mathcal{K})$. The observable algebra of the composite system is then simply given by the tensor product of $\mathcal{A}$ and $\mathcal{B}$, i.e.

$$
\mathcal{A} \otimes \mathcal{B}:=\operatorname{span}\{A \otimes B \mid A \in \mathcal{A}, B \in \mathcal{B}\} \subset \mathcal{B}(\mathcal{K} \otimes \mathcal{H})
$$

The dual of $\mathcal{A} \otimes \mathcal{B}$ is generated by product states, $(\rho \otimes \sigma)(A \otimes B)=\rho(A) \sigma(B)$ and we therefore write $\mathcal{A}^{*} \otimes \mathcal{B}^{*}$ for $(\mathcal{A} \otimes \mathcal{B})^{*}$.

The interpretation of the composed system $\mathcal{A} \otimes \mathcal{B}$ in terms of states and effects is straightforward and therefore postponed to the next Subsection. We will consider first the special cases arising from different choices for $\mathcal{A}$ and $\mathcal{B}$. If both systems are quantum $(\mathcal{A}=\mathcal{B}(\mathcal{H})$ and $\mathcal{B}=\mathcal{B}(\mathcal{K}))$ we get

$$
\mathcal{B}(\mathcal{H}) \otimes \mathcal{B}(\mathcal{K})=\mathcal{B}(\mathcal{H} \otimes \mathcal{K})
$$

as expected. For two classical systems $\mathcal{A}=\mathcal{C}(X)$ and $\mathcal{B}=\mathcal{C}(Y)$ recall that elements of $\mathrm{C}(X)$ (respectively $\mathrm{C}(Y)$ ) are complex valued functions on $X$ (on $Y$ ). Hence the tensor product $\mathrm{C}(X) \otimes \mathcal{C}(Y)$ consists of complex valued functions on $X \times Y$, i.e. $\mathcal{C}(X) \otimes \mathfrak{C}(Y)=\mathfrak{C}(X \times Y)$. In other words states and observables of the composite system $\mathcal{C}(X) \otimes \mathcal{C}(Y)$ are, in accordance with classical probability theory, given by probability distributions and random variables on the Cartesian product $X \times Y$. 
If only one subsystem is classical and the other is quantum; e.g. a micro particle interacting with a classical measuring device we have a hybrid system. The elements of its observable algebra $\mathcal{C}(X) \otimes \mathcal{B}(\mathcal{H})$ can be regarded as operator valued functions on $X$, i.e. $X \ni x \mapsto A_{x} \in \mathcal{B}(\mathcal{H})$ and $A$ is an effect iff $0 \leq A_{x} \leq \mathbb{I}$ holds for all $x \in X$. The elements of the dual $\mathcal{C}^{*}(X) \otimes \mathcal{B}^{*}(\mathcal{H})$ are in a similar way $\mathcal{B}^{*}(X)$ valued functions $X \ni x \mapsto \rho_{x} \in \mathcal{B}^{*}(\mathcal{H})$ and $\rho$ is a state iff each $\rho_{x}$ is a positive trace class operator on $\mathcal{H}$ and $\sum_{x} \rho_{x}=\mathbb{1}$. The probability to measure the effect $A$ in the state $\rho$ is $\sum_{x} \rho_{x}\left(A_{x}\right)$.

2.2.3. Correlations and entanglement. - Let us now consider two effects $A \in \mathcal{A}$ and $B \in \mathcal{B}$ then $A \otimes B$ is an effect of the composite system $\mathcal{A} \otimes \mathcal{B}$. It is interpreted as the joint measurement of $A$ on the first and $B$ on the second subsystem, where the "yes" outcome means "both effects give yes". In particular $A \otimes \mathbb{I}$ means to measure $A$ on the first subsystem and to ignore the second one completely. If $\rho$ is a state of $\mathcal{A} \otimes \mathcal{B}$ we can define its restrictions by $\rho^{\mathcal{A}}(A)=\rho(A \otimes \mathbb{I})$ and $\rho^{\mathcal{B}}(A)=\rho(\mathbb{I} \otimes A)$. If both systems are quantum the restrictions of $\rho$ are the partial traces, while in the classical case we have to sum over the $\mathcal{B}$, respectively $\mathcal{A}$ variables. For two states $\rho_{1} \in \mathcal{S}(\mathcal{A})$ and $\rho_{2} \in \mathcal{S}(\mathcal{B})$ there is always a state $\rho$ of $\mathcal{A} \otimes \mathcal{B}$ such that $\rho_{1}=\rho^{\mathcal{A}}$ and $\rho_{2}=\rho^{\mathcal{B}}$ holds: We just have to choose the product state $\rho_{1} \otimes \rho_{2}$. However in general we have $\rho \neq \rho^{\mathcal{A}} \otimes \rho^{\mathcal{B}}$ which means nothing else then $\rho$ also contains correlations between the two subsystems systems.

Definition 2.2.3 A state $\rho$ of a bipartite system $\mathcal{A} \otimes \mathcal{B}$ is called correlated if there are some $A \in \mathcal{A}, B \in \mathcal{B}$ such that $\rho(A \otimes B) \neq \rho^{\mathcal{A}}(A) \rho^{\mathcal{B}}(B)$ holds.

We immediately see that $\rho=\rho_{1} \otimes \rho_{2}$ implies $\rho(A \otimes B)=\rho_{1}(A) \rho_{2}(B)=$ $\rho^{\mathcal{A}}(A) \rho^{\mathcal{B}}(B)$ hence $\rho$ is not correlated. If on the other hand $\rho(A \otimes B)=\rho^{\mathcal{A}}(A) \rho^{\mathcal{B}}(B)$ holds we get $\rho=\rho^{\mathcal{A}} \otimes \rho^{\mathcal{B}}$. Hence, the definition of correlations just given perfectly fits into our intuitive considerations.

An important issue in quantum information theory is the comparison of correlations between quantum systems on the one hand and classical systems on the other. Hence let us have a closer look on the state space of a system consisting of at least one classical subsystem.

Proposition 2.2.4 Each state $\rho$ of a composite system $\mathcal{A} \otimes \mathcal{B}$ consisting of a classical $(\mathcal{A}=\mathcal{C}(X))$ and an arbitrary system $(\mathcal{B})$ has the form

$$
\rho=\sum_{j \in X} \lambda_{j} \rho_{j}^{\mathcal{A}} \otimes \rho_{j}^{\mathcal{B}}
$$

with positive weights $\lambda_{j}>0$ and $\rho_{j}^{\mathcal{A}} \in \mathcal{S}(\mathcal{A}), \rho_{j}^{\mathcal{B}} \in \mathcal{S}(\mathcal{B})$.

Proof. Since $\mathcal{A}=\mathfrak{C}(X)$ is classical, there is a basis $|j\rangle\langle j| \in \mathcal{A}, j \in X$ of mutually orthogonal one-dimensional projectors and we can write each $A \in \mathcal{A}$ as $\sum_{j} a_{j}|j\rangle\langle j|$ (cf. Subsection 2.1.3). For each state $\rho \in \mathcal{S}\left(\mathcal{A} \otimes \mathcal{B}\right.$ ) we can now define $\rho_{j}^{\mathcal{A}} \in \mathcal{S}(\mathcal{A})$ with $\rho_{j}^{\mathcal{A}}(A)=\operatorname{tr}(A|j\rangle\langle j|)=a_{j}$ and $\rho_{j}^{\mathcal{B}} \in \mathcal{S}(\mathcal{B})$ with $\rho_{j}^{\mathcal{B}}(B)=\lambda_{j}^{-1} \rho(|j\rangle\langle j| \otimes B)$ and $\lambda_{j}=\rho(|j\rangle\langle j| \otimes \mathbb{I})$. Hence we get $\rho=\sum_{j \in X} \lambda_{j} \rho_{j}^{\mathcal{A}} \otimes \rho_{j}^{\mathcal{B}}$ with positive $\lambda_{j}$ as stated.

If $\mathcal{A}$ and $\mathcal{B}$ are two quantum systems it is still possible for them to be correlated in the way just described. We can simply prepare them with a classical random generator which triggers two preparation devices to produce systems in the states $\rho_{j}^{A}, \rho_{j}^{B}$ with probability $\lambda_{j}$. The overall state produced by this setup is obviously the $\rho$ from Equation (2.11). However, the crucial point is that not all correlations of quantum systems are of this type! This is an immediate consequence of the definition of pure states $\rho=|\Psi\rangle\langle\Psi| \in \mathcal{S}(\mathcal{H})$ : Since there is no proper convex decomposition of 
$\rho$, it can be written as in Proposition 2.2.4 iff $\Psi$ is a product vector, i.e. $\Psi=\phi \otimes \psi$. This observation motivates the following definition.

Definition 2.2.5 A state $\rho$ of the composite system $\mathcal{B}\left(\mathcal{H}_{1}\right) \otimes \mathcal{B}\left(\mathcal{H}_{2}\right)$ is called separable or classically correlated if it can be written as

$$
\rho=\sum_{j} \lambda_{j} \rho_{j}^{(1)} \otimes \rho_{j}^{(2)}
$$

with states $\rho_{j}^{(k)}$ of $\mathcal{B}\left(\mathcal{H}_{k}\right)$ and weights $\lambda_{j}>0$. Otherwise $\rho$ is called entangled. The set of all separable states is denoted by $\mathcal{D}\left(\mathcal{H}_{1} \otimes \mathcal{H}_{2}\right)$ or just $\mathcal{D}$ if $\mathcal{H}_{1}$ and $\mathcal{H}_{2}$ are understood.

2.2.4. Bell inequalities. - We have just seen that it is quite easy for pure states to check whether they are entangled or not. In the mixed case however this is a much bigger, and in general unsolved, problem. In this subsection we will have a short look at Bell inequalities, which are maybe the oldest criterion for entanglement (for a more detailed review see [164). Today more powerful methods, most of them based on positivity properties, are available. We will postpone the corresponding discussion to the end of the following section, after we have studied (completely) positive maps (cf. Section 2.4).

Bell inequalities are traditionally discussed in the framework of "local hidden variable theories". More precisely we will say that a state $\rho$ of a bipartite system $\mathcal{B}(\mathcal{H} \otimes \mathcal{K})$ admits a hidden variable model, if there is a probability space $(X, \mu)$ and (measurable) response functions $X \ni x \mapsto F_{A}(x, k), F_{B}(x, l) \in \mathbb{R}$ for all discrete PV measures $A=A_{1}, \ldots, A_{N} \in \mathcal{B}(\mathcal{H})$ respectively $B=B_{1}, \ldots, B_{M} \in \mathcal{B}(\mathcal{K})$ such that

$$
\int_{X} F_{A}(x, k) F_{B}(x, l) \mu(d x)=\operatorname{tr}\left(\rho A_{k} \otimes B_{l}\right)
$$

holds for all, $k, l$ and $A, B$. The value of the functions $F_{A}(x, k)$ is interpreted as the probability to get the value $k$ during an $A$ measurement with known "hidden parameter" $x$. The set of states admitting a hidden variable model is a convex set and as such it can be described by an (infinite) hierarchy of correlation inequalities. Any one of these inequalities is usually called (generalized) Bell inequality. The most well known one is those given by Clauser, Horne, Shimony and Holt 447: The state $\rho$ satisfies the CHSH-inequality if

$$
\rho\left(A \otimes\left(B+B^{\prime}\right)+A^{\prime} \otimes\left(B-B^{\prime}\right)\right) \leq 2
$$

holds for all $A, A^{\prime} \in \mathcal{B}(\mathcal{H})$ respectively $B, B^{\prime} \in \mathcal{B}(\mathcal{K})$, with $-\mathbb{I} \leq A, A^{\prime} \leq \mathbb{I}$ and $-\mathbb{I} \leq B, B^{\prime} \leq \mathbb{I}$. For the special case of two dichotomic observables the CHSH inequalities are sufficient to characterize the states with a hidden variable model. In the general case the CHSH-inequalities are a necessary but not a sufficient condition and a complete characterization is not known.

It is now easy to see that each separable state $\rho=\sum_{j=1}^{n} \lambda_{j} \rho_{j}^{(1)} \otimes \rho_{j}^{(2)}$ admits a hidden variable model: we have to choose $X=1, \ldots, n, \mu(\{j\})=\lambda_{j}$, $F_{A}(x, k)=\rho_{x}^{(1)}\left(A_{k}\right)$ and $F_{B}$ analogously. Hence we immediately see that each state of a composite system with at least one classical subsystem satisfies the Bell inequalities (in particular the CHSH version) while this is not the case for pure quantum systems. The most prominent examples are "maximally entangled states" (cf. Subsection 3.1.1) which violate the CHSH inequality (for appropriately chosen $\left.A, A^{\prime}, B, B^{\prime}\right)$ with a maximal value of $2 \sqrt{2}$. This observation is the starting point for many discussions concerning the interpretation of quantum mechanics, in particular because the maximal violation of $2 \sqrt{2}$ was observed in 1982 experimentally by Aspect and coworkers [5]. We do not want to follow this path (see [164] and the 
the references therein instead). Interesting for us is the fact that Bell inequalities, in particular the CHSH case in Equation (2.14), provide a necessary condition for a state $\rho$ to be separable. However there exist entangled states admitting a hidden variable model 166. Hence, Bell inequalities are not sufficient for separability.

\subsection{Channels}

Assume now that we have a number of quantum systems, e.g. a string of ions in a trap. To "process" the quantum information they carry we have to perform in general many steps of a quite different nature. Typical examples are: free time evolution, controlled time evolution (e.g. the application of a "quantum gate" in a quantum computer), preparations and measurements. The purpose of this section is to provide a unified framework for the description of all these different operations. The basic idea is to represent each processing step by a "channel", which converts input systems, described by an observable algebra $\mathcal{A}$ into output systems described by a possibly different algebra $\mathcal{B}$. Henceforth we will call $\mathcal{A}$ the input and $\mathcal{B}$ the output algebra. If we consider e.g. the free time evolution, we need quantum systems of the same type on the input and the output side, hence in this case we have $\mathcal{A}=\mathcal{B}=\mathcal{B}(\mathcal{H})$ with an appropriately chosen Hilbert space $\mathcal{H}$. If on the other hand we want to describe a measurement we have to map quantum systems (the measured system) to classical information (the measuring result). Therefore we need in this example $\mathcal{A}=\mathcal{B}(\mathcal{H})$ for the input and $\mathcal{B}=\mathfrak{C}(X)$ for the output algebra, where $X$ is the set of possible outcomes of the measurement (cf. Subsection 2.1.4).

Our aim is now to get a mathematical object which can be used to describe a channel. To this end consider an effect $A \in \mathcal{B}$ of the output system. If we invoke first a channel which transforms $\mathcal{A}$ systems into $\mathcal{B}$ systems, and measure $A$ afterwards on the output systems, we end up with a measurement of an effect $T(A)$ on the input systems. Hence we get a map $T: \mathcal{E}(\mathcal{B}) \rightarrow \mathcal{E}(\mathcal{A})$ which completely describes the channel . Alternatively we can look at the states and interpret a channel as a map $T^{*}: \mathcal{S}(\mathcal{A}) \rightarrow \mathcal{S}(\mathcal{B})$ which transforms $\mathcal{A}$ systems in the state $\rho \in \mathcal{S}(\mathcal{A})$ into $\mathcal{B}$ systems in the state $T^{*}(\rho)$. To distinguish between both maps we can say that $T$ describes the channel in the Heisenberg picture and $T^{*}$ in the Schrödinger picture. On the level of the statistical interpretation both points of view should coincide of course, i.e. the probabilities $5\left(T^{*} \rho\right)(A)$ and $\rho(T A)$ to get the result "yes" during an $A$ measurement on $\mathcal{B}$ systems in the state $T^{*} \rho$, respectively a $T A$ measurement on $\mathcal{A}$ systems in the state $\rho$, should be the same. Since $\left(T^{*} \rho\right)(A)$ is linear in $A$ we see immediately that $T$ must be an affine map, i.e. $T\left(\lambda_{1} A_{1}+\lambda_{2} A_{2}\right)=\lambda_{1} T\left(A_{1}\right)+\lambda_{2} T\left(A_{2}\right)$ for each convex linear combination $\lambda_{1} A_{1}+\lambda_{2} A_{2}$ of effects in $\mathcal{B}$, and this in turn implies that $T$ can be extended naturally to a linear map, which we will identify in the following with the channel itself, i.e. we say that $T$ is the channel.

2.3.1. Completely positive maps. - Let us change now slightly our point of view and start with a linear operator $T: \mathcal{A} \rightarrow \mathcal{B}$. To be a channel, $T$ must map effects to effects, i.e. $T$ has to be positive: $T(A) \geq 0 \forall A \geq 0$ and bounded from above by $\mathbb{I}$, i.e. $T(\mathbb{I}) \leq \mathbb{I}$. In addition it is natural to require that two channels in parallel are again a channel. More precisely, if two channels $T: \mathcal{A}_{1} \rightarrow \mathcal{B}_{1}$ and $S: \mathcal{A}_{2} \rightarrow \mathcal{B}_{2}$ are given we can consider the map $T \otimes S$ which associates to each $A \otimes B \in \mathcal{A}_{1} \otimes \mathcal{A}_{2}$ the tensor product $T(A) \otimes S(B) \in \mathcal{B}_{1} \otimes \mathcal{B}_{2}$. It is natural to assume that $T \otimes S$ is a channel which converts composite systems of type $\mathcal{A}_{1} \otimes \mathcal{A}_{2}$ into $\mathcal{B}_{1} \otimes \mathcal{B}_{2}$ systems. Hence $S \otimes T$ should be positive as well 125$]$.

\footnotetext{
${ }^{4}$ Note that the direction of the mapping arrow is reversed compared to the natural ordering of processing.

${ }^{5}$ To keep notations more readable we will follow frequently the usual convention to drop the parenthesis around arguments of linear operators. Hence we will write $T A$ and $T^{*} \rho$ instead of $T(A)$ and $T^{*}(\rho)$. Similarly we will simply write $T S$ instead of $T \circ S$ for compositions.
} 
Definition 2.3.1 Consider two observable algebras $\mathcal{A}, \mathcal{B}$ and a linear map $T$ : $\mathcal{A} \rightarrow \mathcal{B} \subset \mathcal{B}(\mathcal{H})$.

1. $T$ is called positive if $T(A) \geq 0$ holds for all positive $A \in \mathcal{A}$.

2. $T$ is called completely positive (cp) if $T \otimes \mathrm{Id}: \mathcal{A} \otimes \mathcal{B}\left(\mathbb{C}^{n}\right) \rightarrow \mathcal{B}(\mathcal{H}) \otimes \mathcal{B}\left(\mathbb{C}^{n}\right)$ is positive for all $n \in \mathbb{N}$. Here Id denotes the identity map on $\mathcal{B}\left(\mathbb{C}^{n}\right)$.

3. $T$ is called unital if $T(\mathbb{I})=\mathbb{I}$ holds.

Consider now the map $T^{*}: \mathcal{B}^{*} \rightarrow \mathcal{A}^{*}$ which is dual to $T$, i.e. $T^{*} \rho(A)=\rho(T A)$ for all $\rho \in \mathcal{B}^{*}$ and $A \in \mathcal{A}$. It is called the Schrödinger picture representation of the channel $T$, since it maps states to states provided $T$ is unital. (Complete) positivity can be defined in the Schrödinger picture as in the Heisenberg picture and we immediately see that $T$ is (completely) positive iff $T^{*}$ is.

It is natural to ask whether the distinction between positivity and complete positivity is really necessary, i.e. whether there are positive maps which are not completely positive. If at least one of the algebras $\mathcal{A}$ or $\mathcal{B}$ is classical the answer is no: each positive map is completely positive in this case. If both algebras are quantum however complete positivity is not implied by positivity alone. We will discuss explicit examples in Subsection 2.4.2.

If item 2 holds only for a fixed $n \in \mathbb{N}$ the map $T$ is called $n$-positive. This is obviously a weaker condition then complete positivity. However, $n$-positivity implies $m$-positivity for all $m \leq n$, and for $\mathcal{A}=\mathcal{B}\left(\mathbb{C}^{d}\right)$ complete positivity is implied by $n$-positivity, provided $n \geq d$ holds.

Let us consider now the question whether a channel should be unital or not. We have already mentioned that $T(\mathbb{I}) \leq \mathbb{I}$ must hold since effects should be mapped to effects. If $T(\mathbb{I})$ is not equal to $\mathbb{I}$ we get $\rho(T \mathbb{I})=T^{*} \rho(\mathbb{I})<1$ for the probability to measure the effect $\mathbb{I}$ on systems in the state $T^{*} \rho$, but this is impossible for channels which produce an output with certainty, because $\mathbb{I}$ is the effect which is always true. In other words: If a cp map is not unital it describes a channel which sometimes produces no output at all and $T(\mathbb{I})$ is the effect which measures whether we have got an output. We will assume in the future that channels are unital if nothing else is explicitly stated.

2.3.2. The Stinespring theorem. - Consider now channels between quantum systems, i.e. $\mathcal{A}=\mathcal{B}\left(\mathcal{H}_{1}\right)$ and $\mathcal{B}=\mathcal{B}\left(\mathcal{H}_{2}\right)$. A fairly simple example (not necessarily unital) is given in terms of an operator $V: \mathcal{H}_{1} \rightarrow \mathcal{H}_{2}$ by $\mathcal{B}\left(\mathcal{H}_{1}\right) \ni A \mapsto V A V^{*} \in$ $\mathcal{B}\left(\mathcal{H}_{2}\right)$. A second example is the restriction to a subsystem, which is given in the Heisenberg picture by $\mathcal{B}(\mathcal{H}) \ni A \mapsto A \otimes \mathbb{I}_{\mathcal{K}} \in \mathcal{B}(\mathcal{H} \otimes \mathcal{K})$. Finally the composition $S \circ T=S T$ of two channels is again a channel. The following theorem, which is the most fundamental structural result about cp maps ${ }^{\text {f }}$, says that each channel can be represented as a composition of these two examples [147].

Theorem 2.3.2 (StinesPring Dilation theOREM) Every completely positive map $T: \mathcal{B}\left(\mathcal{H}_{1}\right) \rightarrow \mathcal{B}\left(\mathcal{H}_{2}\right)$ has the form

$$
T(A)=V^{*}\left(A \otimes \mathbb{I}_{\mathcal{K}}\right) V,
$$

with an additional Hilbert space $\mathcal{K}$ and an operator $V: \mathcal{H}_{2} \rightarrow \mathcal{H}_{1} \otimes \mathcal{K}$. Both (i.e. $\mathcal{K}$ and $V)$ can be chosen such that the span of all $(A \otimes \mathbb{I}) V \phi$ with $A \in \mathcal{B}\left(\mathcal{H}_{1}\right)$ and $\phi \in \mathcal{H}_{2}$ is dense in $\mathcal{H}_{1} \otimes \mathcal{K}$. This particular decomposition is unique (up to unitary equivalence) and called the minimal decomposition. If $\operatorname{dim} \mathcal{H}_{1}=d_{1}$ and $\operatorname{dim} \mathcal{H}_{2}=d_{2}$ the minimal $\mathcal{K}$ satisfies $\operatorname{dim} \mathcal{K} \leq d_{1}^{2} d_{2}$.

\footnotetext{
${ }^{6}$ Basically there is a more general version of this theorem which works with arbitrary output algebras. It needs howeyer some material from representation theory of $\mathrm{C}^{*}$-algebras which we want to avoid here. See e.g. 125, 83.
} 
By introducing a family $\left|\chi_{j}\right\rangle\left\langle\chi_{j}\right|$ of one dimensional projectors with $\sum_{j}\left|\chi_{j}\right\rangle\left\langle\chi_{j}\right|=\mathbb{I}$ we can define the "Kraus operators" $\left\langle\psi, V_{j} \phi\right\rangle=\left\langle\psi \otimes \chi_{j}, V \phi\right\rangle$. In terms of them we can rewrite Equation (2.15) in the following form 105]:

Corollary 2.3.3 (Kraus Form) Every completely positive map $T: \mathcal{B}\left(\mathcal{H}_{1}\right) \rightarrow$ $\mathcal{B}\left(\mathcal{H}_{2}\right)$ can be written in the form

$$
T(A)=\sum_{j=1}^{N} V_{j}^{*} A V_{j}
$$

with operators $V_{j}: \mathcal{H}_{2} \rightarrow \mathcal{H}_{1}$ and $N \leq \operatorname{dim}\left(\mathcal{H}_{1}\right) \operatorname{dim}\left(\mathcal{H}_{2}\right)$.

2.3.3. The duality lemma. - We will consider a fundamental relation between positive maps and bipartite systems, which will allow us later on to translate properties of entangled states to properties of channels and vice versa. The basic idea originates from elementary linear algebra: A bilinear form $\phi$ on a $d$-dimensional vector space $V$ can be represented by a $d \times d$-matrix, just as an operator on $V$. Hence, we can transform $\phi$ into an operator simply by reinterpreting the matrix elements. In our situation things are more difficult, because the positivity constraints for states and channels should match up in the right way. Nevertheless we have the following theorem.

THEOREM 2.3.4 Let $\rho$ be a density operator on $\mathcal{H} \otimes \mathcal{H}_{1}$. Then there is a Hilbert space $\mathcal{K}$ a pure state $\sigma$ on $\mathcal{H} \otimes \mathcal{K}$ and a channel $T: \mathcal{B}\left(\mathcal{H}_{1}\right) \rightarrow \mathcal{B}(\mathcal{K})$ with

$$
\rho=\left(\operatorname{Id} \otimes T^{*}\right) \sigma,
$$

where Id denotes the identity map on $\mathcal{B}^{*}(\mathcal{H})$. The pure state $\sigma$ can be chosen such that $\operatorname{tr}_{\mathcal{H}}(\sigma)$ has no zero eigenvalue. In this case $T$ and $\sigma$ are uniquely determined (up to unitary equivalence) by Equation (2.17); i.e. if $\widetilde{\sigma}, \widetilde{T}$ with $\rho=\left(\operatorname{Id} \otimes \widetilde{T}^{*}\right) \widetilde{\sigma}$ are given, we have $\widetilde{\sigma}=(\mathbb{I} \otimes U)^{*} \sigma(\mathbb{I} \otimes U)$ and $\widetilde{T}(\cdot)=U^{*} T(\cdot) U$ with an appropriate unitary operator $U$.

Proof. The state $\sigma$ is obviously the purification of $\operatorname{tr}_{\mathcal{H}_{1}}(\rho)$. Hence if $\lambda_{j}$ and $\psi_{j}$ are eigenvalues and eigenvectors of $\operatorname{tr}_{\mathcal{H}_{1}}(\rho)$ we can set $\sigma=|\Psi\rangle\langle\Psi|$ with $\Psi=\sum_{j} \sqrt{\lambda_{j}} \psi_{j} \otimes \phi_{j}$ where $\phi_{j}$ is an (arbitrary) orthonormal basis in $\mathcal{K}$. It is clear that $\sigma$ is uniquely determined up to a unitary. Hence we only have to show that a unique $T$ exists if $\Psi$ is given. To satisfy Equation (2.17) we must have

$$
\begin{aligned}
\rho\left(\left|\psi_{j} \otimes \eta_{k}\right\rangle\left\langle\psi_{l} \otimes \eta_{l}\right|\right) & =\left\langle\Psi,(\operatorname{Id} \otimes T)\left(\left|\psi_{j} \otimes \eta_{k}\right\rangle\left\langle\psi_{l} \otimes \eta_{l}\right|\right) \Psi\right\rangle \\
& =\left\langle\Psi, \mid \psi_{j}\right\rangle\left\langle\psi_{l} \mid \otimes T\left(\left|\eta_{k}\right\rangle\left\langle\eta_{p}\right|\right) \Psi\right\rangle \\
& =\sqrt{\lambda_{j} \lambda_{l}}\left\langle\phi_{j}, T\left(\left|\eta_{k}\right\rangle\left\langle\eta_{p}\right|\right) \phi_{l}\right\rangle,
\end{aligned}
$$

where $\eta_{k}$ is an (arbitrary) orthonormal basis in $\mathcal{H}_{1}$. Hence $T$ is uniquely determined by $\rho$ in terms of its matrix elements and we only have to check complete positivity. To this end it is useful to note that the map $\rho \mapsto T$ is linear if the $\lambda_{j}$ are fixed. Hence it is sufficient to consider the case $\rho=|\chi\rangle\langle\chi|$. Inserting this in Equation (2.20) we immediately see that $T(A)=V^{*} A V$ with $\left\langle V \phi_{j}, \eta_{k}\right\rangle=\lambda_{j}^{-1 / 2}\left\langle\psi_{j} \otimes \eta_{k}, \chi\right\rangle$ holds. Hence $T$ is completely positive. Since normalization $T(\mathbb{I})=\mathbb{I}$ follows from the choice of the $\lambda_{j}$ the theorem is proved.

\subsection{Separability criteria and positive maps}

We have already stated in Subsection 2.3.1 that positive but not completely positive maps exist, whenever input and output algebra are quantum. No such map 
represents a valid quantum operation, nevertheless they are of great importance in quantum information theory, due to their deep relations to entanglement properties. Hence, this Section is a continuation of the study of separability criteria which we have started in 2.2.4. In contrast to the rest of this section, all maps are considered in the Schrödinger rather than in the Heisenberg picture.

2.4.1. Positivity. - Let us consider now an arbitrary positive, but not necessarily completely positive map $T^{*}: \mathcal{B}^{*}(\mathcal{H}) \rightarrow \mathcal{B}^{*}(\mathcal{K})$. If Id again denotes the identity map, it is easy to see that $\left(\operatorname{Id} \otimes T^{*}\right)\left(\sigma_{2} \otimes \sigma_{2}\right)=\sigma_{1} \otimes T^{*}\left(\sigma_{2}\right) \geq 0$ holds for each product state $\sigma_{1} \otimes \sigma_{2} \in \mathcal{S}(\mathcal{H} \otimes \mathcal{K})$. Hence $\left(I d \otimes T^{*}\right) \rho \geq 0$ for each positive $T^{*}$ is a necessary condition for $\rho$ to be separable. The following theorem proved in 86 . shows that sufficiency holds as well.

THEOREM 2.4.1 A state $\rho \in \mathcal{B}^{*}(\mathcal{H} \otimes \mathcal{K})$ is separable iff for any positive map $T^{*}$ : $\mathcal{B}^{*}(\mathcal{K}) \rightarrow \mathcal{B}^{*}(\mathcal{H})$ the operator $\left(\operatorname{Id} \otimes T^{*}\right) \rho$ is positive.

Proof. We will only give a sketch of the proof see 86 for details. The condition is obviously necessary since $\left(\operatorname{Id} \otimes T^{*}\right) \rho_{1} \otimes \rho_{2} \geq 0$ holds for any product state provided $T^{*}$ is positive. The proof of sufficiency relies on the fact that it is always possible to separate a point $\rho$ (an entangled state) from a convex set $\mathcal{D}$ (the set of separable states) by a hyperplane. A precise formulation of this idea leads to the following proposition.

Proposition 2.4.2 For any entangled state $\rho \in \mathcal{S}(\mathcal{H} \otimes \mathcal{K})$ there is an operator $A$ on $\mathcal{H} \otimes \mathcal{K}$ called entanglement witness for $\rho$, with the property $\rho(A)<0$ and $\sigma(A) \geq 0$ for all separable $\sigma \in \mathcal{S}(\mathcal{H} \otimes \mathcal{K})$.

Proof. Since $\mathcal{D} \subset \mathcal{B}^{*}(\mathcal{H} \otimes \mathcal{K})$ is a closed convex set, for each $\rho \in \mathcal{S} \subset \mathcal{B}^{*}(\mathcal{H} \otimes \mathcal{K})$ with $\rho \notin \mathcal{D}$ there exists a linear functional $\alpha$ on $\mathcal{B}^{*}(\mathcal{H} \otimes \mathcal{K})$, such that $\alpha(\rho)<\gamma \leq \alpha(\sigma)$ for each $\sigma \in \mathcal{D}$ with a constant $\gamma$. This holds as well in infinite dimensional Banach spaces and is a consequence of the Hahn-Banach theorem (cf. 135] Theorem 3.4). Without loss of generality we can assume that $\gamma=0$ holds. Otherwise we just have to replace $\alpha$ by $\alpha-\gamma$ tr. Hence the result follows from the fact that each linear functional on $\mathcal{B}^{*}(\mathcal{H} \otimes \mathcal{K})$ has the form $\alpha(\sigma)=\operatorname{tr}(A \sigma)$ with $A \in \mathcal{B}(\mathcal{H} \otimes \mathcal{K})$.

To continue the proof of Theorem 2.4.1 associate now to any operator $A \in$ $\mathcal{B}(\mathcal{H} \otimes \mathcal{K})$ the map $T_{A}^{*}: \mathcal{B}^{*}(\mathcal{K}) \rightarrow \mathcal{B}^{*}(\mathcal{H})$ with

$$
\operatorname{tr}\left(A \rho_{1} \otimes \rho_{2}\right)=\operatorname{tr}\left(\rho_{1}^{T} T_{A}^{*}\left(\rho_{2}\right)\right)
$$

where $(\cdot)^{T}$ denotes the transposition in an arbitrary but fixed orthonormal basis $|j\rangle, j=1, \ldots, d$. It is easy to see that $T_{A}^{*}$ is positive if $\operatorname{tr}\left(A \rho_{1} \otimes \rho_{2}\right) \geq 0$ for all product states $\rho_{1} \otimes \rho_{2} \in \mathcal{S}(\mathcal{H} \otimes \mathcal{K})$ 94]. A straightforward calculation 86] shows in addition that

$$
\operatorname{tr}(A \rho)=\operatorname{tr}\left(|\Psi\rangle\langle\Psi|\left(\operatorname{Id} \otimes T_{A}^{*}\right)(\rho)\right)
$$

holds, where $\Psi=d^{-1 / 2} \sum_{j}|j\rangle \otimes|j\rangle$. Assume now that $\left(\operatorname{Id} \otimes T^{*}\right) \rho \geq 0$ for all positive $T^{*}$. Since $T_{A}^{*}$ is positive this implies that the left hand site of (2.22) is positive, hence $\operatorname{tr}(A \rho) \geq 0$ provided $\operatorname{tr}(A \sigma) \geq 0$ holds for all separable $\sigma$, and the statement follows from Proposition 2.4.2.

2.4.2. The partial transpose. - The most typical example for a positive noncp map is the transposition $\Theta A=A^{T}$ of $d \times d$ matrices, which we have just used in the proof of Theorem 2.4.1. $\Theta$ is obviously a positive map, but the partial transpose

$$
\mathcal{B}^{*}(\mathcal{H} \otimes \mathcal{K}) \ni \rho \mapsto(\operatorname{Id} \otimes \Theta)(\rho) \in \mathcal{B}^{*}(\mathcal{H} \otimes \mathcal{K})
$$


is not. The latter can be easily checked with the maximally entangled state (cf. Subsection 3.1.1).

$$
\Psi=\frac{1}{\sqrt{d}} \sum_{j}|j\rangle \otimes|j\rangle
$$

where $|j\rangle \in \mathbb{C}^{d}, j=1, \ldots, d$ denote the canonical basis vectors. In low dimensions the transposition is basically the only positive map which is not cp. Due to results of Størmer 148 and Woronowicz 174 we have: $\operatorname{dim} \mathcal{H}=2$ and $\operatorname{dim} \mathcal{K}=2,3$ imply that each positive map $T^{*}: \mathcal{B}^{*}(\mathcal{H}) \rightarrow \mathcal{B}^{*}(\mathcal{K})$ has the form $T^{*}=T_{1}^{*}+T_{2}^{*} \Theta$ with two cp maps $T_{1}^{*}, T_{2}^{*}$ and the transposition on $\mathcal{B}(\mathcal{H})$. This immediately implies that positivity of the partial transpose is necessary and sufficient for separability of a state $\rho \in \mathcal{S}(\mathcal{H} \otimes \mathcal{K})($ cf. 86]):

TheOREm 2.4.3 Consider a bipartite system $\mathcal{B}(\mathcal{H} \otimes \mathcal{K})$ with $\operatorname{dim} \mathcal{H}=2$ and $\operatorname{dim} \mathcal{K}=2,3$. A state $\rho \in \mathcal{S}(\mathcal{H} \otimes \mathcal{K})$ is separable iff its partial transpose is positive.

To use positivity of the partial transpose as a separability criterion was proposed for the first time by Peres [127], and he conjectured that it is a necessary and sufficient condition in arbitrary finite dimension. Although it has turned out in the meantime that this conjecture is wrong in general (cf. Subsection 3.1.5), partial transposition has become a crucial tool within entanglement theory and we define:

Definition 2.4.4 A state $\rho \in \mathcal{B}^{*}(\mathcal{H} \otimes \mathcal{K})$ of a bipartite quantum system is called ppt-state if $(\operatorname{Id} \otimes \Theta) \rho \geq 0$ holds and npt-state otherwise (ppt= "positive partial transpose" and npt="negative partial transpose").

2.4.3. The reduction criterion. - Another frequently used example of a noncp but positive map is $\mathcal{B}^{*}(\mathcal{H}) \ni \rho \mapsto T^{*}(\rho)=(\operatorname{tr} \rho) \mathbb{I}-\rho \in \mathcal{B}^{*}(\mathcal{H})$. The eigenvalues of $T^{*}(\rho)$ are given by $\operatorname{tr} \rho-\lambda_{i}$, where $\lambda_{i}$ are the eigenvalues of $\rho$. If $\rho \geq 0$ we have $\lambda_{i} \geq 0$ and therefore $\sum_{j} \lambda_{j}-\lambda_{k} \geq 0$. Hence $T^{*}$ is positive. That $T^{*}$ is not completely positive follows if we consider again the example $|\psi\rangle\langle\psi|$ from Equation (2.24), hence we get

$$
\mathbb{I} \otimes \operatorname{tr}_{2}(\rho)-\rho \geq 0, \quad \operatorname{tr}_{1}(\rho) \otimes \mathbb{I}-\rho \geq 0
$$

for any separable state $\rho \in \mathcal{B}^{*}(\mathcal{H} \otimes \mathcal{K})$, These equations are another non-trivial separability criterion, which is called the reduction criterion [85, 12]. It is closely related to the ppt criterion, due to the following proposition (see [85]) for a proof).

Proposition 2.4.5 Each ppt-state $\rho \in \mathcal{S}(\mathcal{H} \otimes \mathcal{K})$ satisfies the reduction criterion. If $\operatorname{dim} \mathcal{H}=2$ and $\operatorname{dim} \mathcal{K}=2,3$ both criteria are equivalent.

Hence we see with Theorem 2.4.3 that a state $\rho$ in $2 \times 2$ or $2 \times 3$ dimensions is separable iff it satisfies the reduction criterion. 


\section{Chapter 3}

\section{Basic examples}

After the somewhat abstract discussion in the last chapter we will become more concrete now. In the following we will present a number of examples which help on the one hand to understand the structures just introduced, and which are of fundamental importance within quantum information on the other.

\subsection{Entanglement}

Although our definition of entanglement (Definition 2.2.5) is applicable in arbitrary dimensions, detailed knowledge about entangled states is available only for low dimensional systems or for states with very special properties. In this section we will discuss some of the most basic examples.

3.1.1. Maximally entangled states. - Let us start with a look on pure states of a composite systems $\mathcal{A} \otimes \mathcal{B}$ and their possible correlations. If one subsystem is classical, i.e. $\mathcal{A}=\mathcal{C}(\{1, \ldots, d\})$, the state space is given according to Subsection 2.2 .2 by $\mathcal{S}(\mathcal{B})^{d}$ and $\rho \in \mathcal{S}(\mathcal{B})^{d}$ is pure iff $\rho=\left(\delta_{j 1} \tau, \ldots, \delta_{j d} \tau\right)$ with $j=1, \ldots, d$ and a pure state $\tau$ of the $\mathcal{B}$ system. Hence the restrictions of $\rho$ to $\mathcal{A}$ respectively $\mathcal{B}$ are the Dirac measure $\delta_{j} \in \mathcal{S}(X)$ or $\tau \in \mathcal{S}(\mathcal{B})$, in other words both restrictions are pure. This is completely different if $\mathcal{A}$ and $\mathcal{B}$ are quantum, i.e. $\mathcal{A} \otimes \mathcal{B}=\mathcal{B}(\mathcal{H} \otimes \mathcal{K})$ : Consider $\rho=|\Psi\rangle\langle\Psi|$ with $\Psi \in \mathcal{H} \otimes \mathcal{K}$ and Schmidt decomposition (Proposition 2.2.1) $\Psi=\sum_{j} \lambda_{j}^{1 / 2} \phi_{j} \otimes \psi_{j}$. Calculating the $\mathcal{A}$ restriction, i.e. the partial trace over $\mathcal{K}$ we get

$$
\operatorname{tr}\left[\operatorname{tr}_{\mathcal{K}}(\rho) A\right]=\operatorname{tr}[|\Psi\rangle\langle\Psi| A \otimes \mathbb{I}]=\sum_{j k} \lambda_{j}^{1 / 2} \lambda_{k}^{1 / 2}\left\langle\phi_{j}, A \phi_{k}\right\rangle \delta_{j k},
$$

hence $\operatorname{tr}_{\mathcal{K}}(\rho)=\sum_{j} \lambda_{j}\left|\phi_{j}\right\rangle\left\langle\phi_{j}\right|$ is mixed iff $\Psi$ is entangled. The most extreme case arises if $\mathcal{H}=\mathcal{K}=\mathbb{C}^{d}$ and $\operatorname{tr}_{\mathcal{K}}(\rho)$ is maximally mixed, i.e. $\operatorname{tr}_{\mathcal{K}}(\rho)=\frac{\mathbb{I}}{d}$. We get for $\Psi$

$$
\Psi=\frac{1}{\sqrt{d}} \sum_{j=1}^{d} \phi_{j} \otimes \psi_{j}
$$

with two orthonormal bases $\phi_{1}, \ldots, \phi_{d}$ and $\psi_{1}, \ldots, \psi_{d}$. In $2 n \times 2 n$ dimensions these states violate maximally the CHSH inequalities, with appropriately chosen operators $A, A^{\prime}, B, B^{\prime}$. Such states are therefore called maximally entangled. The most prominent examples of maximally entangled states are the four "Bell states" for two qubit systems, i.e. $\mathcal{H}=\mathcal{K}=\mathbb{C}^{2},|1\rangle,|0\rangle$ denotes the canonical basis and

$$
\Phi_{0}=\frac{1}{2}(|11\rangle+|00\rangle), \quad \Phi_{j}=i\left(\mathbb{I} \otimes \sigma_{j}\right) \Phi_{0}, \quad j=1,2,3
$$

where we have used the shorthand notation $|j k\rangle$ for $|j\rangle \otimes|k\rangle$ and the $\sigma_{j}$ denote the Pauli matrices.

The Bell states, which form an orthonormal basis of $\mathbb{C}^{2} \otimes \mathbb{C}^{2}$, are the best studied and most relevant examples of entangled states within quantum information. A mixture of them, i.e. a density matrix $\rho \in \mathcal{S}\left(\mathbb{C}^{2} \otimes \mathbb{C}^{2}\right)$ with eigenvectors $\Phi_{j}$ and eigenvalues $0 \leq \lambda_{j} \leq 1, \sum_{j} \lambda_{j}=1$ is called a Bell diagonal state. It can be shown [16] that $\rho$ is entangled iff $\max _{j} \lambda_{j}>1 / 2$ holds. We omit the proof of this statement here, but we will come back to this point in Chapter 5 within the discussion of entanglement measures. 
Let us come back to the general case now and consider an arbitrary $\rho \in \mathcal{S}(\mathcal{H} \otimes \mathcal{H})$. Using maximally entangled states, we can introduce another separability criterion in terms of the maximally entangled fraction (cf. [16])

$$
\mathcal{F}(\rho)=\sup _{\Psi \text { max. ent. }}\langle\Psi, \rho \Psi\rangle .
$$

If $\rho$ is separable the reduction criterion (2.25) implies $\left\langle\Psi\right.$, $\left.\left[\operatorname{tr}_{1}(\rho) \otimes \mathbb{I}-\rho\right] \Psi\right\rangle \geq 0$ for any maximally entangled state. Since the partial trace of $|\Psi\rangle\langle\Psi|$ is $d^{-1} \mathbb{I}$ we get

$$
d^{-1}=\left\langle\Psi, \operatorname{tr}_{1}(\rho) \otimes \mathbb{I} \Psi\right\rangle \leq\langle\Psi, \rho \Psi\rangle,
$$

hence $\mathcal{F}(\rho) \leq 1 / d$. This condition is not very sharp however. Using the ppt criterion it can be shown that $\rho=\lambda\left|\Phi_{1}\right\rangle\left\langle\Phi_{1}|+(1-\lambda)| 00\right\rangle\langle 00|$ (with the Bell state $\Phi_{1}$ ) is entangled for all $0<\lambda \leq 1$ but a straightforward calculation shows that $\mathcal{F}(\rho) \leq 1 / 2$ holds for $\lambda \leq 1 / 2$.

Finally, we have to mention here a very useful parameterization of the set of pure states on $\mathcal{H} \otimes \mathcal{H}$ in terms of maximally entangled states: If $\Psi$ is an arbitrary but fixed maximally entangled state, each $\phi \in \mathcal{H} \otimes \mathcal{H}$ admits (uniquely determined) operators $X_{1}, X_{2}$ such that

$$
\phi=\left(X_{1} \otimes \mathbb{I}\right) \Psi=\left(\mathbb{I} \otimes X_{2}\right) \Psi
$$

holds. This can be easily checked in a product basis.

3.1.2. Werner states. - If we consider entanglement of mixed states rather than pure ones, the analysis becomes quite difficult, even if the dimensions of the underlying Hilbert spaces are low. The reason is that the state space $\mathcal{S}\left(\mathcal{H}_{1} \otimes \mathcal{H}_{2}\right)$ of a two-partite system with $\operatorname{dim} \mathcal{H}_{i}=d_{i}$ is a geometric object in a $d_{1}^{2} d_{2}^{2}-1$ dimensional space. Hence even in the simplest non-trivial case (two qubits) the dimension of the state space becomes very high (15 dimensions) and naive geometric intuition can be misleading. Therefore it is often useful to look at special classes of model states, which can be characterized by only few parameters. A quite powerful tool is the study of symmetry properties; i.e. to investigate the set of states which is invariant under a group of local unitaries. A general discussion of this scheme can be found in 159. In this paper we will present only three of the most prominent examples.

Consider first a state $\rho \in \mathcal{S}(\mathcal{H} \otimes \mathcal{H})$ (with $\mathcal{H}=\mathbb{C}^{d}$ ) which is invariant under the group of all $U \otimes U$ with a unitary $U$ on $\mathcal{H}$; i.e. $[U \otimes U, \rho]=0$ for all $U$. Such a $\rho$ is usually called a Werner state 166, 128 and its structure can be analyzed quite easily using a well known result of group theory which goes back to Weyl [171] (see also Theorem IX.11.5 of [142]), and which we will state in detail for later reference:

THEOREM 3.1.1 Each operator $A$ on the $N$-fold tensor product $\mathcal{H}^{\otimes N}$ of the (finite dimensional) Hilbert space $\mathcal{H}$ which commutes with all unitaries of the form $U^{\otimes N}$ is a linear combination of permutation operators, i.e. $A=\sum_{\pi} \lambda_{\pi} V_{\pi}$, where the sum is taken over all permutations $\pi$ of $N$ elements, $\lambda_{\pi} \in \mathbb{C}$ and $V_{\pi}$ is defined by

$$
V_{\pi} \phi_{1} \otimes \cdots \otimes \phi_{N}=\phi_{\pi^{-1}(1)} \otimes \cdots \otimes \phi_{\pi^{-1}(N)} .
$$

In our case $(N=2)$ there are only two permutations: the identity $\mathbb{I}$ and the flip $F(\psi \otimes \phi)=\phi \otimes \psi$. Hence $\rho=a \mathbb{I}+b F$ with appropriate coefficients $a, b$. Since $\rho$ is a density matrix, $a$ and $b$ are not independent. To get a transparent way to express these constraints, it is reasonable to consider the eigenprojections $P_{ \pm}$of $F$ rather then $\mathbb{I}$ and $F$; i.e. $F P_{ \pm} \psi= \pm P_{ \pm} \psi$ and $P_{ \pm}=(\mathbb{I} \pm F) / 2$. The $P_{ \pm}$are the projections on the subspaces $\mathcal{H}_{ \pm}^{\otimes 2} \subset \mathcal{H} \otimes \mathcal{H}$ of symmetric respectively antisymmetric tensor products (Bose- respectively Fermi-subspace). If we write $d_{ \pm}=d(d \pm 1) / 2$ for the dimensions of $\mathcal{H}_{ \pm}^{\otimes 2}$ we get for each Werner state $\rho$

$$
\rho=\frac{\lambda}{d_{+}} P_{+}+\frac{(1-\lambda)}{d_{-}} P_{-}, \quad \lambda \in[0,1]
$$


On the other hand it is obvious that each state of this form is $U \otimes U$ invariant, hence a Werner state.

If $\rho$ is given, it is very easy to calculate the parameter $\lambda$ from the expectation value of $\rho$ and the flip $\operatorname{tr}(\rho F)=2 \lambda-1 \in[-1,1]$. Therefore we can write for an arbitrary state $\sigma \in \mathcal{S}(\mathcal{H} \otimes \mathcal{H})$

$$
P_{\mathrm{UU}}(\sigma)=\frac{\operatorname{tr}(\sigma F)+1}{2 d_{+}} P_{+}+\frac{(1-\operatorname{tr} \sigma F)}{2 d_{-}} P_{-},
$$

and this defines a projection from the full state space to the set of Werner states which is called the twirl operation. In many cases it is quite useful that it can be written alternatively as a group average of the form

$$
P_{\mathrm{UU}}(\sigma)=\int_{\mathrm{U}(d)}(U \otimes U) \sigma\left(U^{*} \otimes U^{*}\right) d U,
$$

where $d U$ denotes the normalized, left invariant Haar measure on $\mathrm{U}(d)$. To check this identity note first that its right hand side is indeed $U \otimes U$ invariant, due to the invariance of the volume element $d U$. Hence we have to check only that the trace of $F$ times the integral coincides with $\operatorname{tr}(F \sigma)$ :

$$
\begin{aligned}
\operatorname{tr}\left[F \int_{\mathrm{U}(d)}(U \otimes U) \sigma\left(U^{*} \otimes U^{*}\right) d U\right] & =\int_{\mathrm{U}(d)} \operatorname{tr}\left[F(U \otimes U) \sigma\left(U^{*} \otimes U^{*}\right)\right] d U \\
& =\operatorname{tr}(F \sigma) \int_{\mathrm{U}(d)} d U=\operatorname{tr}(F \sigma),
\end{aligned}
$$

where we have used the fact that $F$ commutes with $U \otimes U$ and the normalization of $d U$. We can apply $P_{\mathrm{UU}}$ obviously to arbitrary operators $A \in \mathcal{B}(\mathcal{H} \otimes \mathcal{H})$ and, as an integral over unitarily implemented operations, we get a channel. Substituting $U \rightarrow$ $U^{*}$ in $(3.10)$ and cycling the $\operatorname{trace} \operatorname{tr}\left(A P_{\mathrm{UU}}(\sigma)\right)$ we find $\operatorname{tr}\left(P_{\mathrm{UU}}(A) \rho\right)=\operatorname{tr}\left(A P_{\mathrm{UU}}(\rho)\right)$, hence $P_{\text {UU }}$ has the same form in the Heisenberg and the Schrödinger picture (i.e. $\left.P_{\mathrm{UU}}^{*}=P_{\mathrm{UU}}\right)$.

If $\sigma \in \mathcal{S}(\mathcal{H} \otimes \mathcal{H})$ is a separable state the integrand of $P_{\mathrm{UU}}(\sigma)$ in Equation (3.10) consists entirely of separable states, hence $P_{\mathrm{UU}}(\sigma)$ is separable. Since each Werner state $\rho$ is the twirl of itself, we see that $\rho$ is separable iff it is the twirl $P_{\mathrm{UU}}(\sigma)$ of a separable state $\sigma \in \mathcal{S}(\mathcal{H} \otimes \mathcal{H})$. To determine the set of separable Werner states we therefore have to calculate only the set of all $\operatorname{tr}(F \sigma) \in[-1,1]$ with separable $\sigma$. Since each such $\sigma$ admits a convex decomposition into pure product states it is sufficient to look at

$$
\langle\psi \otimes \phi, F \psi \otimes \phi\rangle=|\langle\psi, \phi\rangle|^{2}
$$

which ranges from 0 to 1 . Hence $\rho$ from Equation (3.8) is separable iff $1 / 2 \leq \lambda \leq 1$ and entangled otherwise (due to $\lambda=(\operatorname{tr}(F \rho)+1) / 2)$. If $\mathcal{H}=\mathbb{C}^{2}$ holds, each Werner state is Bell diagonal and we recover the result from Subsection 3.1.1 (separable if highest eigenvalue less or equal than $1 / 2$ ).

3.1.3. Isotropic states. — To derive a second class of states consider the partial transpose $(\operatorname{Id} \otimes \Theta) \rho$ (with respect to a distinguished base $|j\rangle \in \mathcal{H}, j=1, \ldots, d$ ) of a Werner state $\rho$. Since $\rho$ is, by definition, $U \otimes U$ invariant, it is easy to see that $(\mathrm{Id} \otimes \Theta) \rho$ is $U \otimes \bar{U}$ invariant, where $\bar{U}$ denotes component wise complex conjugation in the base $|j\rangle$ (we just have to use that $U^{*}=\bar{U}^{T}$ holds). Each state $\tau$ with this kind of symmetry is called an isotropic state [132], and our previous discussion shows that $\tau$ is a linear combination of $\mathbb{I}$ and the partial transpose of the flip, which is the rank one operator

$$
\widetilde{F}=(\operatorname{Id} \otimes \Theta) F=|\Psi\rangle\left\langle\Psi\left|=\sum_{j k=1}^{d}\right| j j\right\rangle\langle k k|,
$$


where $\Psi=\sum_{j}|j j\rangle$ is, up to normalization a maximally entangled state. Hence each isotropic $\tau$ can be written as

$$
\tau=\frac{1}{d}\left(\lambda \frac{\mathbb{I}}{d}+(1-\lambda) \widetilde{F}\right), \quad \lambda \in\left[0, \frac{d^{2}}{d^{2}-1}\right],
$$

where the bounds on $\lambda$ follow from normalization and positivity. As above we can determine the parameter $\lambda$ from the expectation value

$$
\operatorname{tr}(\widetilde{F} \tau)=\frac{1-d^{2}}{d} \lambda+d
$$

which ranges from 0 to $d$ and this again leads to a twirl operation: For an arbitrary state $\sigma \in \mathcal{S}(\mathcal{H} \otimes \mathcal{H})$ we can define

$$
P_{\mathrm{UU}}(\sigma)=\frac{1}{d\left(1-d^{2}\right)}([\operatorname{tr}(\widetilde{F} \sigma)-d] \mathbb{I}+[1-d \operatorname{tr}(\widetilde{F} \sigma)] \widetilde{F})
$$

and as for Werner states $P_{\mathrm{UU}}$ can be rewritten in terms of a group average

$$
P_{\mathrm{U} \overline{\mathrm{U}}}(\sigma)=\int_{\mathrm{U}(d)}(U \otimes \bar{U}) \sigma\left(U^{*} \otimes \bar{U}^{*}\right) d U .
$$

Now we can proceed in the same way as above: $P_{\mathrm{UU}}$ is a channel with $P_{\mathrm{UU}}^{*}=P_{\mathrm{UU}}$, its fixed points $P_{\mathrm{UU}}(\tau)=\tau$ are exactly the isotropic states, and the image of the set of separable states under $P_{\mathrm{UU}}$ coincides with the set of separable isotropic states. To determine the latter we have to consider the expectation values (cf. Equation (3.13))

$$
\langle\psi \otimes \phi, \widetilde{F} \psi \otimes \phi\rangle=\left|\sum_{j=1}^{d} \psi_{j} \phi_{j}\right|=|\langle\psi, \bar{\phi}\rangle|^{2} \in[0,1] .
$$

This implies that $\tau$ is separable iff

$$
\frac{d(d-1)}{d^{2}-1} \leq \lambda \leq \frac{d^{2}}{d^{2}-1}
$$

holds and entangled otherwise. For $\lambda=0$ we recover the maximally entangled state. For $d=2$, again we recover again the special case of Bell diagonal states encountered already in the last subsection.

3.1.4. OO-invariant states. - Let us combine now Werner states with isotropic states, i.e. we look for density matrices $\rho$ which can be written as $\rho=a \mathbb{I}+b F+c \widetilde{F}$, or, if we introduce the three mutually orthogonal projection operators

$$
p_{0}=\frac{1}{d} \widetilde{F}, \quad p_{1}=\frac{1}{2}(\mathbb{I}-F), \quad \frac{1}{2}(\mathbb{I}+F)-\frac{1}{d} \widetilde{F}
$$

as a convex linear combination of $\operatorname{tr}\left(p_{j}\right)^{-1} p_{j}, j=0,1,2$ :

$$
\rho=\left(1-\lambda_{1}-\lambda_{2}\right) p_{0}+\lambda_{1} \frac{p_{1}}{\operatorname{tr}\left(p_{1}\right)}+\lambda_{2} \frac{p_{2}}{\operatorname{tr}\left(p_{2}\right)}, \quad \lambda_{1}, \lambda_{2} \geq 0, \lambda_{1}+\lambda_{2} \leq 1
$$

Each such operator is invariant under all transformations of the form $U \otimes U$ if $U$ is a unitary with $U=\bar{U}$, in other words: $U$ should be a real orthogonal matrix. A little bit representation theory of the orthogonal group shows that in fact all operators with this invariance property have the form given in (3.22); cf. [159]. The corresponding states are therefore called $O O$-invariant, and we can apply basically 


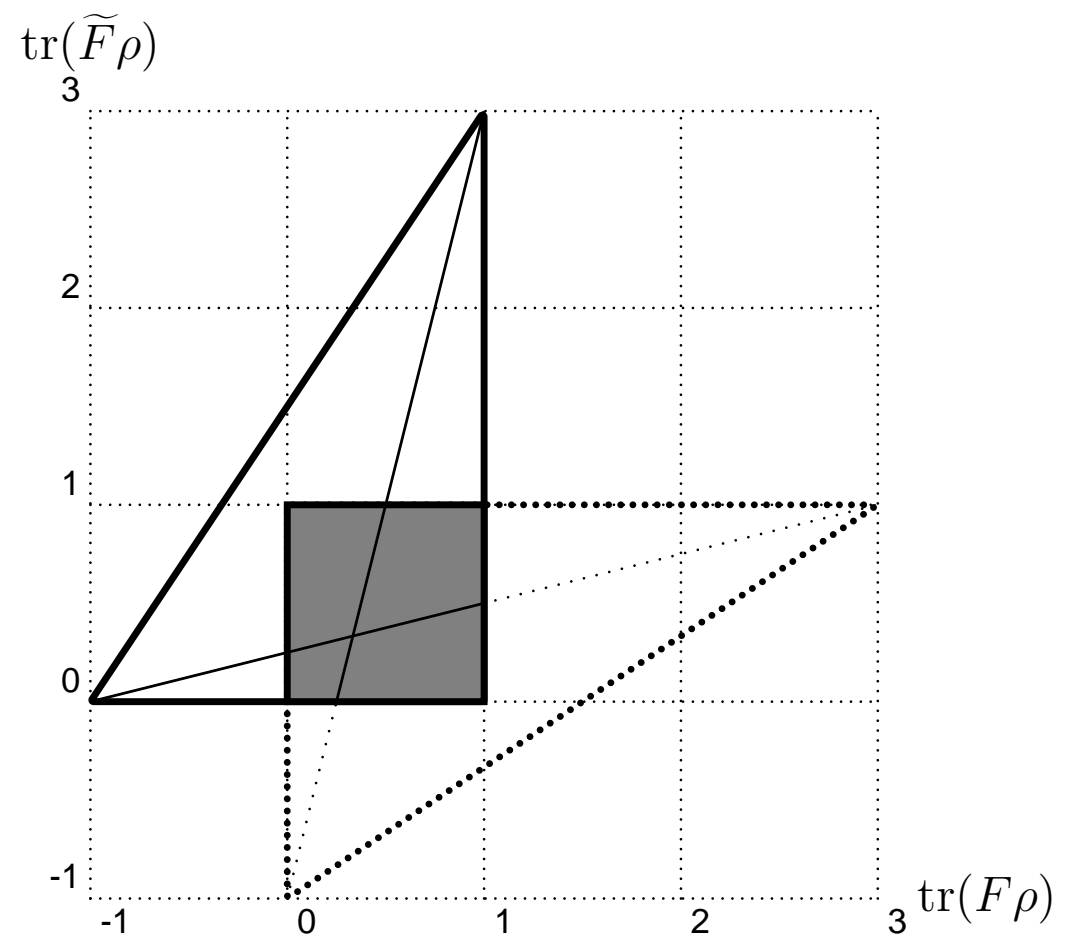

Figure 3.1: State space of OO-invariant states (upper triangle) and its partial transpose (lower triangle) for $d=3$. The special cases of isotropic and Werner states are drawn as thin lines.

the same machinery as in Subsection 3.1.2 if we replace the unitary group $\mathrm{U}(d)$ by the orthogonal group $\mathrm{O}(d)$. This includes in particular the definition of a twirl operation as an average over $\mathrm{O}(d)$ (for an arbitrary $\rho \in \mathcal{S}(\mathcal{H} \otimes \mathcal{H})$ ):

$$
P_{\mathrm{OO}}(\rho)=\int_{\mathrm{O}(d)} U \otimes U \rho U \otimes U^{*} d U
$$

which we can express alternatively in terms of the expectation values $\operatorname{tr}(F \rho), \operatorname{tr}(\widetilde{F} \rho)$ by

$$
P_{\mathrm{OO}}(\rho)=\frac{\operatorname{tr}(\widetilde{F} \rho)}{d} p_{0}+\frac{1-\operatorname{tr}(F \rho)}{2 \operatorname{tr}\left(p_{1}\right)} p_{1}+\left(\frac{1+\operatorname{tr}(F \rho)}{2}-\frac{\operatorname{tr}(\widetilde{F} \rho)}{d}\right) \frac{p_{2}}{\operatorname{tr}\left(p_{2}\right)}
$$

The range of allowed values for $\operatorname{tr}(F \rho), \operatorname{tr}(\widetilde{F} \rho)$ is given by

$$
-1 \leq \operatorname{tr}(F \rho) \leq 1, \quad 0 \leq \operatorname{tr}(\widetilde{F} \rho) \leq d, \quad \operatorname{tr}(F \rho) \geq \frac{2 \operatorname{tr}(\widetilde{F} \rho)}{d}-1 .
$$

For $d=3$ this is the upper triangle in Figure 3.1.

The values in the lower (dotted) triangle belong to partial transpositions of OO-invariant states. The intersection of both, i.e. the gray shaded square $Q=$ $[0,1] \times[0,1]$, represents therefore the set of OO-invariant ppt states, and at the same time the set of separable states, since each OO-invariant ppt state is separable. To see the latter note that separable OO-invariant states form a convex subset of $Q$. Hence, we only have to show that the corners of $Q$ are separable. To do this note 
that 1. $P_{\mathrm{OO}}(\rho)$ is separable whenever $\rho$ is and 2. that $\operatorname{tr}\left(F P_{\mathrm{OO}}(\rho)\right)=\operatorname{tr}(F \rho)$ and $\operatorname{tr}\left(\widetilde{F} P_{\mathrm{OO}}(\rho)\right)=\operatorname{tr}(F \rho)$ holds (cf. Equation (3.12) $)$. We can consider pure product states $|\phi \otimes \psi\rangle\langle\phi \otimes \psi|$ for $\rho$ and get $\left(|\langle\phi, \psi\rangle|^{2},\left.\langle\phi, \bar{\psi}\rangle\right|^{2}\right)$ for the tuple $(\operatorname{tr}(F \rho), \operatorname{tr}(\widetilde{F} \rho))$. Now the point 1,1$)$ in $Q$ is obtained if $\psi=\phi$ is real, the point $(0,0)$ is obtained for real and orthogonal $\phi, \psi$ and the point $(1,0)$ belongs to the case $\psi=\phi$ and $\langle\phi, \bar{\phi}\rangle=0$. Symmetrically we get $(0,1)$ with the same $\phi$ and $\psi=\bar{\phi}$.

3.1.5. PPT states. - We have seen in Theorem 2.4.3 that separable states and ppt states coincide in $2 \times 2$ and $2 \times 3$ dimensions. Another class of examples with this property are OO-invariant states just studied. Nevertheless, separability and a positive partial transpose are not equivalent. An easy way to produce such examples of states which are entangled and ppt is given in terms of unextendible product bases [14]. An orthonormal family $\phi_{j} \in \mathcal{H}_{1} \otimes \mathcal{H}_{2}, j=1, \ldots, N<d_{1} d_{2}$ (with $d_{k}=\operatorname{dim} \mathcal{H}_{k}$ ) is called an unextendible product basis (UPB) iff 1 . all $\phi_{j}$ are product vectors and 2 . there is no product vector orthogonal to all $\phi_{j}$. Let us denote the projector to the span of all $\phi_{j}$ by $E$, its orthocomplement by $E^{\perp}$, i.e. $E^{\perp}=\mathbb{I}-E$, and define the state $\rho=\left(d_{1} d_{2}-N\right)^{-1} E^{\perp}$. It is entangled because there is by construction no product vector in the support of $\rho$, and it is ppt. The latter can be seen as follows: The projector $E$ is a sum of the one dimensional projectors $\left|\phi_{j}\right\rangle\left\langle\phi_{j}\right|, j=1, \ldots, N$. Since all $\phi_{j}$ are product vectors the partial transposes of the $\left|\phi_{j}\right\rangle\left\langle\phi_{j}\right|$ are of the form $\left|\widetilde{\phi}_{j}\right\rangle\left\langle\widetilde{\phi}_{j}\right|$, with another UPB $\widetilde{\phi}_{j}, j=1, \ldots, N$ and the partial transpose $(\mathbb{I} \otimes \Theta) E$ of $E$ is the sum of the $\left|\widetilde{\phi}_{j}\right\rangle\left\langle\widetilde{\phi}_{j}\right|$. Hence $(\mathbb{I} \otimes \Theta) E^{\perp}=\mathbb{I}-(\mathbb{I} \otimes \Theta) E$ is a projector and therefore positive.

To construct entangled ppt states we have to find UPBs. The following two examples are taken from [14]. Consider first the five vectors

$$
\phi_{j}=N(\cos (2 \pi j / 5), \sin (2 \pi j / 5), h), \quad j=0, \ldots, 4,
$$

with $N=2 / \sqrt{5+\sqrt{5}}$ and $h=\frac{1}{2} \sqrt{1+\sqrt{5}}$. They form the apex of a regular pentagonal pyramid with height $h$. The latter is chosen such that nonadjacent vectors are orthogonal. It is now easy to show that the five vectors

$$
\Psi_{j}=\phi_{j} \otimes \phi_{2 j \bmod 5}, \quad j=0, \ldots, 4
$$

form a UPB in the Hilbert space $\mathcal{H} \otimes \mathcal{H}, \operatorname{dim} \mathcal{H}=3$ (cf. [14]). A second example, again in $3 \times 3$ dimensional Hilbert space are the following five vectors (called "Tiles" in [14):

$$
\begin{array}{ll}
\frac{1}{\sqrt{2}}|0\rangle \otimes(|0\rangle-|1\rangle), & \frac{1}{\sqrt{2}}|2\rangle \otimes(|1\rangle-|2\rangle), \quad \frac{1}{\sqrt{2}}(|0\rangle-|1\rangle) \otimes|2\rangle, \\
\frac{1}{\sqrt{2}}(|1\rangle-|2\rangle) \otimes|0\rangle, & \frac{1}{3}(|0\rangle+|1\rangle+|2\rangle) \otimes(|0\rangle+|1\rangle+|2\rangle),
\end{array}
$$

where $|k\rangle, k=0,1,2$ denotes the standard basis in $\mathcal{H}=\mathbb{C}^{3}$.

3.1.6. Multipartite states. - In many applications of quantum information rather big systems, consisting of a large number of subsystems, occur (e.g. a quantum register of a quantum computer) and it is necessary to study the corresponding correlation and entanglement properties. Since this is a fairly difficult task, there is not much known about - much less as in the two-partite case, which we mainly consider in this paper. Nevertheless, in this subsection we will give a rough outline of some of the most relevant aspects.

At the level of pure states the most significant difficulty is the lack of an analog of the Schmidt decomposition 126. More precisely there are elements in an $N$-fold

\footnotetext{
${ }^{1}$ This name is somewhat misleading because the $\phi_{j}$ are not a base of $\mathcal{H}_{1} \otimes \mathcal{H}_{2}$.
} 
tensor product $\mathcal{H}^{(1)} \otimes \cdots \otimes \mathcal{H}^{(N)}$ (with $N>2$ ) which can not be written asฎ

$$
\Psi=\sum_{j=1}^{d} \lambda_{j} \phi_{j}^{(1)} \otimes \cdots \otimes \phi_{j}^{(N)}
$$

with $N$ orthonormal bases $\phi_{1}^{(k)}, \ldots, \phi_{d}^{(k)}$ of $\mathcal{H}^{(k)}, k=1, \ldots, N$. To get examples for such states in the tri-partite case, note first that any partial trace of $|\Psi\rangle\langle\Psi|$ with $\Psi$ from Equation (3.29) has separable eigenvectors. Hence, each purification (Corollary 2.2.2) of an entangled, two-partite, mixed state with inseparable eigenvectors (e.g. a Bell diagonal state) does not admit a Schmidt decomposition. This implies on the one hand that there are interesting new properties to be discovered, but on the other we see that many techniques developed for bipartite pure states can be generalized in a straightforward way only for states which are Schmidt decomposable in the sense of Equation (3.29). The most well known representative of this class for a tripartite qubit system is the GHZ state 73

$$
\Psi=\frac{1}{\sqrt{2}}(|000\rangle+|111\rangle)
$$

which has the special property that contradictions between local hidden variable theories and quantum mechanics occur even for non-statistical predictions (as opposed to maximally entangled states of bipartite systems; 73, 117, 116]).

A second new aspect arising in the discussion of multiparty entanglement is the fact that several different notions of separability occur. A state $\rho$ of an $N$-partite system $\mathcal{B}\left(\mathcal{H}_{1}\right) \otimes \cdots \otimes \mathcal{B}\left(\mathcal{H}_{N}\right)$ is called $N$-separable if

$$
\rho=\sum_{J} \lambda_{J} \rho_{j_{1}} \otimes \cdots \otimes \rho_{j_{N}},
$$

with states $\rho_{j_{k}} \in \mathcal{B}^{*}\left(\mathcal{H}_{k}\right)$ and multi indices $J=\left(j_{1}, \ldots, j_{k}\right)$. Alternatively, however, we can decompose $\mathcal{B}\left(\mathcal{H}_{1}\right) \otimes \cdots \otimes \mathcal{B}\left(\mathcal{H}_{N}\right)$ in two subsystems (or even into $M$ subsystems if $M<N$ ) and call $\rho$ biseparable if it is separable with respect to this decomposition. It is obvious that $N$-separability implies biseparability with respect to all possible decompositions. The converse is - not very surprisingly - not true. One way to construct a corresponding counterexample is to use an unextendable product base (cf. Subsection 3.1.5). In [14] it is shown that the tripartite qubit state complementary to the UPB

$$
|0,1,+\rangle,|1,+, 0\rangle,|+, 0,1\rangle,|-,-,-\rangle \text { with }| \pm\rangle=\frac{1}{\sqrt{2}}(|0\rangle \pm|1\rangle)
$$

is entangled (i.e. tri-inseparable) but biseparable with respect to any decomposition into two subsystems (cf. 14 for details).

Another, maybe more systematic, way to find examples for multipartite states with interesting properties is the generalization of the methods used for Werner states (Subsection 3.1.2), i.e. to look for density matrices $\rho \in \mathcal{B}^{*}\left(\mathcal{H}^{\otimes N}\right)$ which commute with all unitaries of the form $U^{\otimes N}$. Applying again theorem 3.1.1 we see that each such $\rho$ is a linear combination of permutation unitaries. Hence the structure of the set of all $U^{\otimes N}$ invariant states can be derived from representation theory of the symmetric group (which can be tedious for large $N$ !). For $N=3$ this program is carried out in 61 and it turns out that the corresponding set of invariant states is a five dimensional (real) manifold. We skip the details here and refer to 61] instead.

\footnotetext{
${ }^{2}$ There is however the possibility to choose the bases $\phi_{1}^{(k)}, \ldots, \phi_{d}^{(k)}$ such that the number of summands becomes minimal. For tri-partite systems this "minimal canonical form" is study in [1].
} 


\subsection{Channels}

In Section 2.3 we have introduced channels as very general objects transforming arbitrary types of information (i.e. classical, quantum and mixtures of them) into one another. In the following we will consider some of the most important special cases.

3.2.1. Quantum channnels. - Many tasks of quantum information theory require the transmission of quantum information over long distances, using devices like optical fibers or storing quantum information in some sort of memory. Both situations can be described by a channel or quantum operation $T: \mathcal{B}(\mathcal{H}) \rightarrow \mathcal{B}(\mathcal{H})$, where $T^{*}(\rho)$ is the quantum information which will be received when $\rho$ was sent, or alternatively: which will be read off the quantum memory when $\rho$ was written. Ideally we would prefer those channels which do not affect the information at all, i.e. $T=\mathbb{I}$, or, as the next best choice, a $T$ whose action can be undone by a physical device, i.e. $T$ should be invertible and $T^{-1}$ is again a channel. The Stinespring Theorem (Theorem 2.3.2) immediately shows that this implies $T^{*} \rho=U \rho U^{*}$ with a unitary $U$; in other words the systems carrying the information do not interact with the environment. We will call such a kind of channel an ideal channel. In real situations however interaction with the environment, i.e. additional, unobservable degrees of freedom, can not be avoided. The general structure of such a noisy channel is given by

$$
T^{*}(\rho)=\operatorname{tr}_{\mathcal{K}}\left(U\left(\rho \otimes \rho_{0}\right) U^{*}\right)
$$

where $U: \mathcal{H} \otimes \mathcal{K} \rightarrow \mathcal{H} \otimes \mathcal{K}$ is a unitary operator describing the common evolution of the system (Hilbert space $\mathcal{H}$ ) and the environment (Hilbert space $\mathcal{K}$ ) and $\rho_{0} \in \mathcal{S}(\mathcal{K})$ is the initial state of the environment (cf. Figure 3.2). It is obvious that the quantum information originally stored in $\rho \in \mathcal{S}(\mathcal{H})$ can not be completely recovered from $T^{*}(\rho)$ if only one system is available. It is an easy consequence of the Stinepspring theorem that each channel can be expressed in this form

Corollary 3.2.1 (Ancilla Form) Assume that $T: \mathcal{B}(\mathcal{H}) \rightarrow \mathcal{B}(\mathcal{H})$ is a channel. Then there is a Hilbert space $\mathcal{K}$, a pure state $\rho_{0}$ and a unitary map $U: \mathcal{H} \otimes \mathcal{K} \rightarrow$ $\mathcal{H} \otimes \mathcal{K}$ such that Equation (3.33) holds. It is allways possible, to choose $\mathcal{K}$ such that $\operatorname{dim}(\mathcal{K})=\operatorname{dim}(\mathcal{H})^{3}$ holds.

Proof. Consider the Stinepspring form $T(A)=V^{*}(A \otimes \mathbb{I}) V$ with $V: \mathcal{H} \rightarrow \mathcal{H} \otimes \mathcal{K}$ of $T$ and choose a vector $\psi \in \mathcal{K}$ such that $U(\phi \otimes \psi)=V(\phi)$ can be extended to a unitary map $U: \mathcal{H} \otimes \mathcal{K} \rightarrow \mathcal{H} \otimes \mathcal{K}$ (this is always possible since $T$ is unital and $V$ therefore isometric). If $e_{j} \in \mathcal{H}, j=1, \ldots, d_{1}$ and $f_{k} \in \mathcal{K}, k=1, \ldots, d_{2}$ are orthonormal bases with $f_{1}=\psi$ we get

$$
\begin{aligned}
\operatorname{tr}[T(A) \rho] & =\operatorname{tr}\left[\rho V^{*}(A \otimes \mathbb{I}) V\right]=\sum_{j}\left\langle V \rho e_{j},(A \otimes \mathbb{I}) V e_{j}\right\rangle \\
& =\sum_{j k}\left\langle U(\rho \otimes|\psi\rangle\langle\psi|)\left(e_{j} \otimes f_{k}\right),(A \otimes \mathbb{I}) U\left(e_{j} \otimes f_{k}\right)\right\rangle \\
& =\operatorname{tr}\left[\operatorname{tr}_{\mathcal{K}}\left[U(\rho \otimes|\psi\rangle\langle\psi|) U^{*}\right] A\right],
\end{aligned}
$$

which proves the statement.

Note that there are in general many ways to express a channel this way, e.g. if $T$ is an ideal channel $\rho \mapsto T^{*} \rho=U \rho U^{*}$ we can rewrite it with an arbitrary unitary $U_{0}: \mathcal{K} \rightarrow \mathcal{K}$ by $T^{*} \rho=\operatorname{tr}_{2}\left(U \otimes U_{0} \rho \otimes \rho_{0} U^{*} \otimes U_{0}^{*}\right)$. This is the weakness of the ancilla form compared to the Stinespring representation of Theorem 2.3.2. Nevertheless 


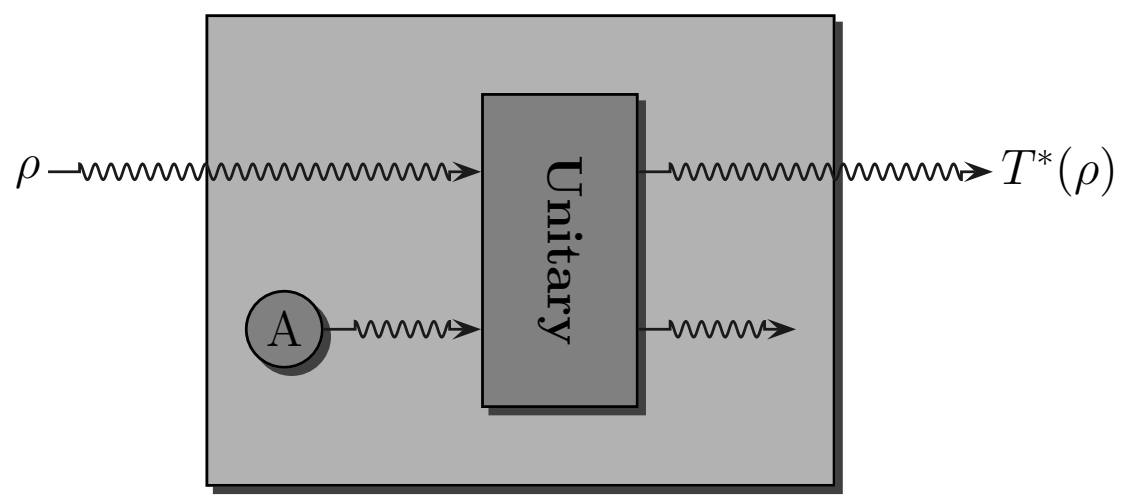

Figure 3.2: Noisy channel

Corollary 3.2.1 shows that each channel which is not an ideal channel is noisy in the described way.

The most prominent example for a noisy channel is the depolarizing channel for d-level systems (i.e. $\mathcal{H}=\mathbb{C}^{d}$ )

$$
\mathcal{S}(\mathcal{H}) \ni \rho \mapsto \vartheta \rho+(1-\vartheta) \frac{\mathbb{I}}{d} \in \mathcal{S}(\mathcal{H}), \quad 0 \leq \vartheta \leq 1
$$

or in the Heisenberg picture

$$
\mathcal{B}(\mathcal{H}) \ni A \mapsto \vartheta A+(1-\vartheta) \frac{\operatorname{tr}(A)}{d} \mathbb{I} \in \mathcal{B}(\mathcal{H}) .
$$

A Stinespring dilation of $T$ (not the minimal one - this can be checked by counting dimensions) is given by $\mathcal{K}=\mathcal{H} \otimes \mathcal{H} \oplus \mathbb{C}$ and $V: \mathcal{H} \rightarrow \mathcal{H} \otimes \mathcal{K}=\mathcal{H} \otimes 3 \oplus \mathcal{H}$ with

$$
|j\rangle \mapsto V|j\rangle=\left[\sqrt{\frac{1-\vartheta}{d}} \sum_{k=1}^{d}|k\rangle \otimes|k\rangle \otimes|j\rangle\right] \oplus[\sqrt{\vartheta}|j\rangle],
$$

where $|k\rangle, k=1, \ldots, d$ denotes again the canonical basis in $\mathcal{H}$. An ancilla form of $T$ with the same $\mathcal{K}$ is given by the (pure) environment state

$$
\psi=\left[\sqrt{\frac{1-\vartheta}{d}} \sum_{k=1}^{d}|k\rangle \otimes|k\rangle\right] \oplus[\sqrt{\vartheta}|0\rangle] \in \mathcal{K}
$$

and the unitary operator $U: \mathcal{H} \otimes \mathcal{K} \rightarrow \mathcal{H} \otimes \mathcal{K}$ with

$$
U\left(\phi_{1} \otimes \phi_{2} \otimes \phi_{3} \oplus \chi\right)=\phi_{2} \otimes \phi_{3} \otimes \phi_{1} \oplus \chi,
$$

i.e. $U$ is the direct sum of a permutation unitary and the identity.

3.2.2. Channels under symmetry. - Similarly to the discussion in Section 3.1 it is often useful to consider channels with special symmetry properties. To be more precise, consider a group $G$ and two unitary representations $\pi_{1}, \pi_{2}$ on the Hilbert spaces $\mathcal{H}_{1}$ and $\mathcal{H}_{2}$ respectively. A channel $T: \mathcal{B}\left(\mathcal{H}_{1}\right) \rightarrow \mathcal{B}\left(\mathcal{H}_{2}\right)$ is called covariant (with respect to $\pi_{1}$ and $\pi_{2}$ ) if

$$
T\left[\pi_{1}(U) A \pi_{1}(U)^{*}\right]=\pi_{2}(U) T[A] \pi_{2}(U)^{*} \quad \forall A \in \mathcal{B}\left(\mathcal{H}_{1}\right) \forall U \in G
$$

holds. The general structure of covariant channels is governed by a fairly powerful variant of Stinesprings theorem which we will state below (and which will be very 
useful for the study of the cloning problem in Chapter 7). Before we do this let us have a short look on a particular class of examples which is closely related to OO-invariant states.

Hence consider a channel $T: \mathcal{B}(\mathcal{H}) \rightarrow \mathcal{B}(\mathcal{H})$ which is covariant with respect to the orthogonal group, i.e. $T\left(U A U^{*}\right)=U T(A) U^{*}$ for all unitaries $U$ on $\mathcal{H}$ with $\bar{U}=U$ in a distinguished basis $|j\rangle, j=1, \ldots, d$. The maximally entangled state $\psi=$ $d^{-1 / 2} \sum_{j}|j j\rangle$ is OO-invariant, i.e. $U \otimes U \psi=\psi$ for all these $U$. Therefore each state $\rho=\left(\operatorname{Id} \otimes T^{*}\right)|\psi\rangle\langle\psi|$ is OO-invariant as well and by the duality lemma (Theorem 2.3.4) $T$ and $\psi$ are uniquely determined (up to unitary equivalence) by $\rho$. This means we can use the structure of OO-invariant states derived in Subsection 3.1.4 to characterize all orthogonal covariant channels. As a first step consider the linear maps $X_{1}(A)=d \operatorname{tr}(A) \mathbb{I}, X_{2}(A)=d A^{T}$ and $X_{3}(A)=d A$. They are not channels (they are not unital and $X_{2}$ is not $\mathrm{cp}$ ) but they have the correct covariance property and it is easy to see that they correspond to the operators $\mathbb{I}, F, \widetilde{F} \in \mathcal{B}(\mathcal{H} \otimes \mathcal{H})$, i.e.

$$
\left(\operatorname{Id} \otimes X_{1}\right)|\psi\rangle\left\langle\psi\left|=\mathbb{I},\left(\operatorname{Id} \otimes X_{2}\right)\right| \psi\right\rangle\left\langle\psi\left|=F,\left(\operatorname{Id} \otimes X_{3}\right)\right| \psi\right\rangle\langle\psi|=\widetilde{F} .
$$

Using Equation (3.21) we can determine therefore the channels which belong to the three extremal OO-invariant states (the corners of the upper triangle in Figure 3.1):

$$
\begin{gathered}
T_{0}(A)=A, T_{1}(A)=\frac{\operatorname{tr}(A) \mathbb{I}-A^{T}}{d-1} \\
T_{2}(A)=\frac{2}{d(d+1)-2}\left[\frac{d}{2}\left(\operatorname{tr}(A) \mathbb{I}+A^{T}\right)-A\right]
\end{gathered}
$$

Each OO-invariant channel is a convex linear combination of these three. Special cases are the channels corresponding to Werner and isotropic states. The latter leads to depolarizing channels $T(A)=\vartheta A+(1-\vartheta) d^{-1} \operatorname{tr}(A) \mathbb{I}$ with $\vartheta \in\left[0, d^{2} /\left(d^{2}-1\right)\right]$; cf. Equation (3.15), while Werner states correspond to

$$
T(A)=\frac{\vartheta}{d+1}\left[\operatorname{tr}(A) \mathbb{I}+A^{T}\right]+\frac{1-\vartheta}{d-1}\left[\operatorname{tr}(A) \mathbb{I}-A^{T}\right], \vartheta \in[0,1] ;
$$

cf. Equation (3.8).

Let us come back now to the general case. We will state here the covariant version of the Stinespring theorem (see 98 for a proof). The basic idea is that all covariant channels are parameterized by representations on the dilation space.

THEOREM 3.2.2 Let $G$ be a group with finite dimensional unitary representations $\pi_{j}: G \rightarrow \mathrm{U}\left(\mathcal{H}_{j}\right)$ and $T: \mathcal{B}\left(\mathcal{H}_{1}\right) \rightarrow \mathcal{B}\left(\mathcal{H}_{2}\right)$ a $\pi_{1}, \pi_{2}$ - covariant channel. Then there is a finite dimensional unitary representation $\widetilde{\pi}: G \rightarrow \mathrm{U}(\mathcal{K})$ and an operator $V: \mathcal{H}_{2} \rightarrow \mathcal{H}_{1} \otimes \mathcal{K}$ with $V \pi_{2}(U)=\pi_{1}(U) \otimes \widetilde{\pi}(U)$ and $T(A)=V^{*} A \otimes \mathbb{I} V$.

To get an explicit example consider the dilation of a depolarizing channel given in Equation (3.39). In this case we have $\pi_{1}(U)=\pi_{2}(U)=U$ and $\widetilde{\pi}(U)=(U \otimes \bar{U}) \oplus \mathbb{I}$. The check that the map $V$ has indeed the intertwining property $V \pi_{2}(U)=\pi_{1}(U) \otimes$ $\tilde{\pi}(U)$ stated in the theorem is left as an exercise to the reader.

3.2.3. Classical channels. - The classical analog to a quantum operation is a channel $T: \mathcal{C}(X) \rightarrow \mathcal{C}(Y)$ which describes the transmission or manipulation of classical information. As we have mentioned already in Subsection 2.3.1 positivity and complete positivity are equivalent in this case. Hence we have to assume only that $T$ is positive and unital. Obviously $T$ is characterized by its matrix elements $T_{x y}=\delta_{y}(T|x\rangle\langle x|)$, where $\delta_{y} \in \mathcal{C}^{*}(X)$ denotes the Dirac measure at $y \in Y$ and $|x\rangle\langle x| \in \mathcal{C}(X)$ is the canonical basis in $\mathrm{e}(X)$ (cf. Subsection 2.1.3). Positivity and normalization of $T$ imply that $0 \leq T_{x y} \leq 1$ and

$$
1=\delta_{y}(\mathbb{I})=\delta_{y}(T(\mathbb{I}))=\delta_{y}\left[T\left(\sum_{x}|x\rangle\langle x|\right)\right]=\sum_{x} T_{x y}
$$


holds. Hence the family $\left(T_{x y}\right)_{x \in X}$ is a probability distribution on $X$ and $T_{x y}$ is therefore the probability to get the information $x \in X$ at the output side of the channel if $y \in Y$ was send. Each classical channel is uniquely determined by its matrix of transition probabilities. For $X=Y$ we see that the information is transmitted without error iff $T_{x y}=\delta_{x y}$, i.e. $T$ is an ideal channel if $T=\operatorname{Id}$ holds and noisy otherwise.

3.2.4. Observables and Preparations. - Let us consider now a channel which transforms quantum information $\mathcal{B}(\mathcal{H})$ into classical information $\mathcal{C}(X)$. Since positivity and complete positivity are again equivalent, we just have to look at a positive and unital map $E: \mathcal{C}(X) \rightarrow \mathcal{B}(\mathcal{H})$. With the canonical basis $|x\rangle\langle x|, x \in X$ of $\mathcal{C}(X)$ we get a family $E_{x}=E(|x\rangle\langle x|), x \in X$ of positive operators $E_{x} \in \mathcal{B}(\mathcal{H})$ with $\sum_{x \in X} E_{x}=\mathbb{I}$. Hence the $E_{x}$ form a POV measure, i.e. an observable. If on the other hand a POV measure $E_{x} \in \mathcal{B}(\mathcal{H}), x \in X$ is given we can define a quantum to classical channel $E: \mathcal{C}(X) \rightarrow \mathcal{B}(\mathcal{H})$ by $E(f)=\sum_{x} f(x) E_{x}$. This shows that the observable $E_{x}, x \in X$ and the channel $E$ can be identified and we say $E$ is the observable.

With this interpretation in mind it is possible to have a short look at continuous observables without the need of abstract measure theory: We only have to say how the classical algebra $\mathcal{C}(X)$ is defined for a set $X$ which is not finite or discrete. For simplicity we assume that $X=\mathbb{R}$ holds, however the generalization to other locally compact spaces is straightforward. We choose for $\mathcal{C}(\mathbb{R})$ the space of continuous, complex valued functions vanishing at infinity, i.e. $|f(x)|<\epsilon$ for each $\epsilon>0$ provided $|x|$ is large enough. $\mathcal{C}(\mathbb{R})$ can be equipped with the sup-norm and becomes an Abelian $\mathrm{C}^{*}$-algebra (cf. [25]). To interpret it as an operator algebra as assumed in Subsection 2.1.1 we have to identify $f \in \mathcal{C}(\mathbb{R})$ with the corresponding multiplication operator on $\mathrm{L}^{2}(\mathbb{R})$. An observable taking arbitrary real values can be defined now as a positive map $E: \mathcal{C}(\mathbb{R}) \rightarrow \mathcal{B}(\mathcal{H})$. The probability to get a result in the interval $[a, b] \subset \mathbb{R}$ during an $E$ measurement on systems in the state $\rho$ is $\beta$

$$
\mu([a, b])=\sup \{\operatorname{tr}(E(f) \rho) \mid f \in \mathcal{C}(\mathbb{R}), 0 \leq f \leq \mathbb{I}, \operatorname{supp} f \subset[a, b]\}
$$

where supp denotes the support of $f$. The most well known example for $\mathbb{R}$ valued observables are of course position $Q$ and momentum $P$ of a free particle in one dimension. In this case we have $\mathcal{H}=\mathrm{L}^{2}(\mathbb{R})$ and the channels corresponding to $Q$ and $P$ are (in position representation) given by $\mathcal{C}(\mathbb{R}) \ni f \mapsto E_{Q}(f) \in \mathcal{B}(\mathcal{H})$ with $E_{Q}(f) \psi=f \psi$ respectively $\mathcal{C}(\mathbb{R}) \ni f \mapsto E_{P}(f) \in \mathcal{B}(\mathcal{H})$ with $E_{P}(f) \psi=(f \widehat{\psi})^{\vee}$ where $\wedge$ and $\vee$ denote the Fourier transform and its inverse.

Let us return now to a finite set $X$ and exchange the role of $\mathcal{C}(X)$ and $\mathcal{B}(\mathcal{H})$; in other words let us consider a channel $R: \mathcal{B}(\mathcal{H}) \rightarrow \mathcal{C}(X)$ with a classical input and a quantum output algebra. In the Schrödinger picture we get a family of density matrices $\rho_{x}:=R^{*}\left(\delta_{x}\right) \in \mathcal{B}^{*}(\mathcal{H}), x \in X$, where $\delta_{x} \in \mathcal{C}^{*}(X)$ denote again the Dirac measures (cf. Subsection 2.1.3). Hence we get a parameter dependent preparation which can be used to encode the classical information $x \in X$ into the quantum information $\rho_{x} \in \mathcal{B}^{*}(\mathcal{H})$.

3.2.5. Instruments and Parameter Dependent Operations. - An observable describes only the statistics of measuring results, but contains no information about the state of the system after the measurement. To get a description which fills this gap we have to consider channels which operates on quantum systems and produces hybrid systems as output, i.e. $T: \mathcal{B}(\mathcal{H}) \otimes \mathcal{M}(X) \rightarrow \mathcal{B}(\mathcal{K})$. Following Davies [50] we will call such an object an instrument. From $T$ we can derive the subchannel

$$
\mathcal{C}(X) \ni f \mapsto T(\mathbb{I} \otimes f) \in \mathcal{B}(\mathcal{K})
$$

\footnotetext{
${ }^{3}$ Due to the Riesz-Markov theorem (cf. Theorem IV.18 of 134) the set function $\mu$ extends in unique way to a probability measure on the real line.
} 
which is the observable measured by $T$, i.e. $\operatorname{tr}[T(\mathbb{I} \otimes|x\rangle\langle x|) \rho]$ is the probability to measure $x \in X$ on systems in the state $\rho$. On the other hand we get for each $x \in X$ a quantum channel (which is not unital)

$$
\mathcal{B}(\mathcal{H}) \ni A \mapsto T_{x}(A)=T(A \otimes|x\rangle\langle x|) \in \mathcal{B}(\mathcal{K}) .
$$

It describes the operation performed by the instrument $T$ if $x \in X$ was measured. More precisely if a measurement on systems in the state $\rho$ gives the result $x \in X$ we get (up to normalization) the state $T_{x}^{*}(\rho)$ after the measurement (cf. Figure 3.3), while

$$
\operatorname{tr}\left(T_{x}^{*}(\rho)\right)=\operatorname{tr}\left(T_{x}^{*}(\rho) \mathbb{I}\right)=\operatorname{tr}(\rho T(\mathbb{I} \otimes|x\rangle\langle x|))
$$

is (again) the probability to measure $x \in X$ on $\rho$. The instrument $T$ can be expressed in terms of the operations $T_{x}$ by

$$
T(A \otimes f)=\sum_{x} f(x) T_{x}(A) ;
$$

hence we can identify $T$ with the family $T_{x}, x \in X$. Finally we can consider the second marginal of $T$

$$
\mathcal{B}(\mathcal{H}) \ni A \mapsto T(A \otimes \mathbb{I})=\sum_{x \in X} T_{x}(A) \in \mathcal{B}(\mathcal{K}) .
$$

It describes the operation we get if the outcome of the measurement is ignored.

The most well known example of an instrument is a von Neumann-Lüders measurement associated to a PV measure given by family of projections $E_{x}, x=1, \ldots d$; e.g. the eigenprojections of a selfadjoint operator $A \in \mathcal{B}(\mathcal{H})$. It is defined as the channel

$$
T: \mathcal{B}(\mathcal{H}) \otimes \mathfrak{C}(X) \rightarrow \mathcal{B}(\mathcal{H}) \text { with } X=\{1, \ldots, d\} \text { and } T_{x}(A)=E_{x} A E_{x}
$$

Hence we get the final state $\operatorname{tr}\left(E_{x} \rho\right)^{-1} E_{x} \rho E_{x}$ if we measure the value $x \in X$ on systems initially in the state $\rho$ - this is well known from quantum mechanics.

Let us change now the role of $\mathcal{B}(\mathcal{H}) \otimes \mathcal{C}(X)$ and $\mathcal{B}(\mathcal{K})$; in other words consider a channel $T: \mathcal{B}(\mathcal{K}) \rightarrow \mathcal{B}(\mathcal{H}) \otimes \mathcal{C}(X)$ with hybrid input and quantum output. It describes a device which changes the state of a system depending on additional classical information. As for an instrument, $T$ decomposes into a family of (unital!) channels $T_{x}: \mathcal{B}(\mathcal{K}) \rightarrow \mathcal{B}(\mathcal{H})$ such that we get $T^{*}(\rho \otimes p)=\sum_{x} p_{x} T_{x}^{*}(\rho)$ in the Schrödinger picture. Physically $T$ describes a parameter dependent operation: depending on the classical information $x \in X$ the quantum information $\rho \in \mathcal{B}(\mathcal{K})$ is transformed by the operation $T_{x}$ (cf. figure 3.4)

Finally we can consider a channel $T: \mathcal{B}(\mathcal{H}) \otimes \mathcal{C}(X) \rightarrow \mathcal{B}(\mathcal{K}) \otimes \mathcal{C}(Y)$ with hybrid input and output to get a parameter dependent instrument (cf. figure 3.5): Similarly

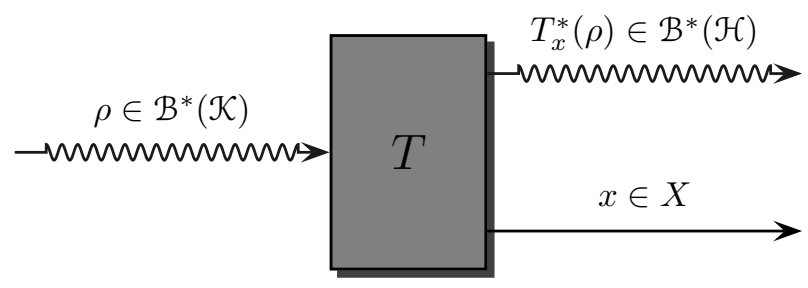

Figure 3.3: Instrument 
to the discussion in the last paragraph we can define a family of instruments $T_{y}$ : $\mathcal{B}(\mathcal{H}) \otimes \mathcal{C}(X) \rightarrow \mathcal{B}(\mathcal{K}), y \in Y$ by the equation $T^{*}(\rho \otimes p)=\sum_{y} p_{y} T_{y}^{*}(\rho)$. Physically $T$ describes the following device: It receives the classical information $y \in Y$ and a quantum system in the state $\rho \in \mathcal{B}^{*}(\mathcal{K})$ as input. Depending on $y$ a measurement with the instrument $T_{y}$ is performed, which in turn produces the measuring value $x \in X$ and leaves the quantum system in the state (up to normalization) $T_{y, x}^{*}(\rho)$; with $T_{y, x}$ given as in Equation (3.50) by $T_{y, x}(A)=T_{y}(A \otimes|x\rangle\langle x|)$.

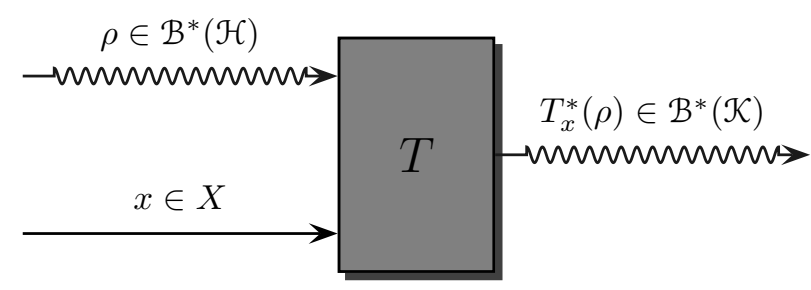

Figure 3.4: Parameter dependent operation

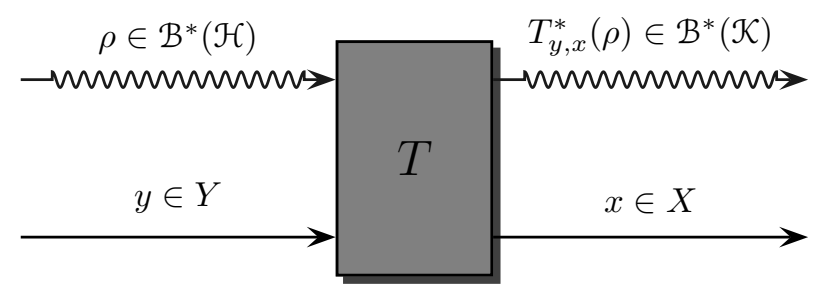

Figure 3.5: Parameter dependent instrument

3.2.6. LOCC and separable channels. - Let us consider now channels acting on finite dimensional bipartite systems: $T: \mathcal{B}\left(\mathcal{H}_{1} \otimes \mathcal{K}_{2}\right) \rightarrow \mathcal{B}\left(\mathcal{K}_{1} \otimes \mathcal{K}_{2}\right)$. In this case we can ask the question whether a channel preserves separability. Simple examples are local operations (LO), i.e. $T=T^{A} \otimes T^{B}$ with two channels $T^{A, B}: \mathcal{B}\left(\mathcal{H}_{j}\right) \rightarrow$ $\mathcal{B}\left(\mathcal{K}_{j}\right)$. Physically we think of such a $T$ in terms of two physicists Alice and Bob both performing operations on their own particle but without information transmission neither classical nor quantum. The next difficult step are local operations with one way classical communications (one way LOCC). This means Alice operates on her system with an instrument, communicates the classical measuring result $j \in X=\{1, \ldots, N\}$ to Bob and he selects an operation depending on these data. We can write such a channel as a composition $T=\left(T^{A} \otimes \operatorname{Id}\right)\left(\operatorname{Id} \otimes T^{B}\right)$ of the instrument $T^{A}: \mathcal{B}\left(\mathcal{H}_{1}\right) \otimes \mathcal{C}\left(X_{1}\right) \rightarrow \mathcal{B}\left(\mathcal{K}_{1}\right)$ and the parameter dependent operation $T^{B}: \mathcal{B}\left(\mathcal{H}_{2}\right) \rightarrow \mathfrak{C}\left(X_{1}\right) \otimes \mathcal{B}\left(\mathcal{K}_{2}\right)$ (cf. Figure 3.6)

$$
\mathcal{B}\left(\mathcal{H}_{1} \otimes \mathcal{H}_{2}\right) \stackrel{\operatorname{Id} \otimes T^{B}}{\longrightarrow} \mathcal{B}\left(\mathcal{H}_{1}\right) \otimes \mathcal{C}(X) \otimes \mathcal{B}\left(\mathcal{K}_{2}\right) \stackrel{T^{A} \otimes \mathrm{Id}}{\longrightarrow} \mathcal{B}\left(\mathcal{K}_{1} \otimes \mathcal{K}_{2}\right) .
$$

It is of course possible to continue the chain in Equation (3.55), i.e. instead of just operating on his system, Bob can invoke a parameter dependent instrument depending on Alice's data $j_{1} \in X_{1}$, send the corresponding measuring results $j_{2} \in X_{2}$ to Alice and so on. To write down the corresponding chain of maps (as in Equation (3.55) ) is simple but not very illuminating and therefore omitted; cf. Figure 3.7 instead. If we allow Alice and Bob to drop some of their particles, i.e. the operations they perform need not to be unital, we get a $L O C C$ channel ("local operations and classical communications"). It represents the most general physical process which can be performed on a two partite system if only classical communication (in both directions) is available. 


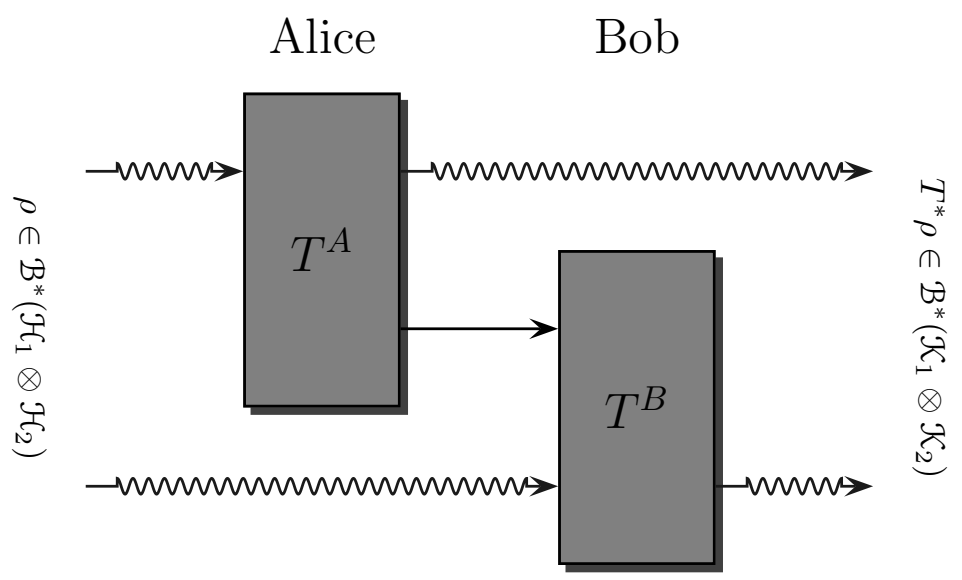

Figure 3.6: One way LOCC operation; cf Figure 3.7 for an explanation.

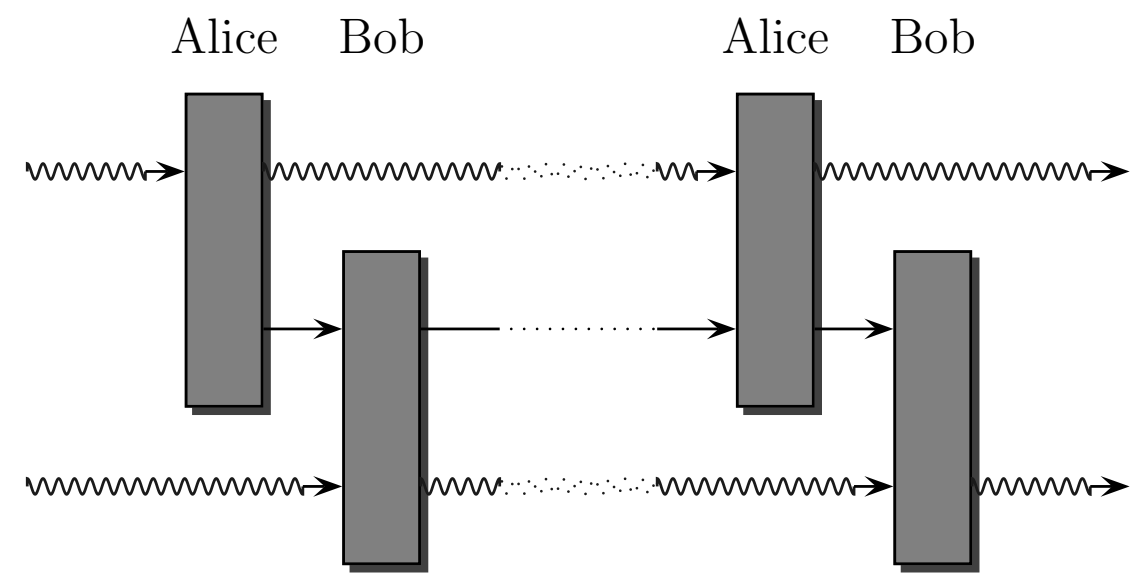

Figure 3.7: LOCC operation. The upper and lower curly arrows represent Alice's respectively Bob's quantum system, while the straight arrows in the middle stand for the classical information Alice and Bob exchange. The boxes symbolize the channels applied by Alice and Bob.

LOCC channels play a significant role in entanglement theory (we will see this in Section 4.3), but they are difficult to handle. Fortunately it is often possible to replace them by closely related operations with a more simple structure: A not necessarily unital channel $T: \mathcal{B}\left(\mathcal{H}_{1} \otimes \mathcal{K}_{2}\right) \rightarrow \mathcal{B}\left(\mathcal{K}_{1} \otimes \mathcal{K}_{2}\right)$ is called separable, if it is a sum of (in general non-unital) local operations, i.e.

$$
T=\sum_{j=1}^{N} T_{j}^{A} \otimes T_{j}^{B} .
$$

It is easy to see that a separable $T$ maps separable states to separable states (up to normalization) and that each LOCC channel is separable (cf. [13]). The converse however is (somewhat surprisingly) not true: there are separable channels which are not LOCC, see [13] for a concrete example.

\subsection{Quantum mechanics in phase space}

Up to now we have considered only finite dimensional systems and even in this extremely idealized situation it is not easy to get nontrivial results. At a first look 
the discussion of continuous quantum systems seems therefore to be hopeless. If we restrict our attention however to small classes of states and channels, with sufficiently simple structure, many problems become tractable. Phase space quantum mechanics, which will be reviewed in this Section (see Chapter 5 of 79 for details), provides a very powerful tool in this context.

Before we start let us add some remarks to the discussion of Chapter 2 which we have restricted to finite dimensional Hilbert spaces. Basically most of the material considered there can be generalized in a straightforward way, as long as topological issues like continuity and convergence arguments are treated carefully enough. There are of course some caveats (cf. in particular Footnote 2 of Chapter 2), however they do not lead to problems in the framework we are going to discuss and can therefore be ignored.

3.3.1. Weyl operators and the CCR. - The kinematical structure of a quantum system with $d$ degrees of freedom is usually described by a separable Hilbert space $\mathcal{H}$ and $2 d$ selfadjoint operators $Q_{1}, \ldots, Q_{d}, P_{1}, \ldots, P_{d}$ satisfying the canonical commutation relations $\left[Q_{j}, Q_{k}\right]=0,\left[P_{j}, P_{k}\right]=0,\left[Q_{j}, P_{k}\right]=i \delta_{j k} \mathbb{I}$. The latter can be rewritten in a more compact form as

$$
R_{2 j-1}=Q_{j}, R_{2 j}=P_{j}, j=1, \ldots, d,\left[R_{j}, R_{k}\right]=-i \sigma_{j k}
$$

Here $\sigma$ denotes the symplectic matrix

$$
\sigma=\operatorname{diag}(J, \ldots, J), \quad J=\left[\begin{array}{cc}
0 & 1 \\
-1 & 0
\end{array}\right]
$$

which plays a crucial role for the geometry of classical mechanics. We will call the pair $(V, \sigma)$ consisting of $\sigma$ and the $2 d$-dimensional real vector space $V=\mathbb{R}^{2 d}$ henceforth the classical phase space.

The relations in Equation (3.57) are, however, not sufficient to fix the operators $R_{j}$ up to unitary equivalence. The best way to remove the remaining physical ambiguities is the study of the unitaries

$$
W(x)=\exp (i x \cdot \sigma \cdot R), x \in V, x \cdot \sigma \cdot R=\sum_{j k=1}^{2 d} x_{j} \sigma_{j k} R_{k}
$$

instead of the $R_{j}$ directly. If the family $W(x), x \in V$ is irreducible (i.e. $[W(x), A]=$ $0, \forall x \in V$ implies $A=\lambda \mathbb{I}$ with $\lambda \in \mathbb{C}$ ) and satisfies

$$
W(x) W\left(x^{\prime}\right)=\exp \left(-\frac{i}{2} x \cdot \sigma \cdot x^{\prime}\right) W\left(x+x^{\prime}\right)
$$

it is called an (irreducible) representation of the Weyl relations (on $(V, \sigma)$ ) and the operators $W(x)$ are called Weyl operators. By the well known Stone - von Neumann uniqueness theorem all these representations are mutually unitarily equivalent, i.e. if we have two of them $W_{1}(x), W_{2}(x)$, there is a unitary operator $U$ with $U W_{1}(x) U^{*}=$ $W_{2}(x) \forall x \in V$. This implies that it does not matter from a physical point of view which representation we use. The most well known one is of course the Schrödinger representation where $\mathcal{H}=\mathrm{L}^{2}\left(\mathbb{R}^{d}\right)$ and $Q_{j}, P_{k}$ are the usual position and momentum operators.

\footnotetext{
${ }^{4}$ Note that the CCR (3.57) are implied by the Weyl relations 3.60 but the converse is, in contrast to popular believe, not true: There are representations of the CCR which are unitarily inequivalent to the Schrödinger representation; cf. 134 Section VIII.5 for particular examples. Hence uniqueness can only be achieved on the level of Weyl operators - which is one major reason to study them.
} 
3.3.2. Gaussian states. - A density operator $\rho \in \mathcal{S}(\mathcal{H})$ has finite second moments if the expectation values $\operatorname{tr}\left(\rho Q_{j}^{2}\right)$ and $\operatorname{tr}\left(\rho P_{j}^{2}\right)$ are finite for all $j=1, \ldots, d$. In this case we can define the mean $m \in \mathbb{R}^{2 d}$ and the correlation matrix $\alpha$ by

$$
m_{j}=\operatorname{tr}(\rho R), \quad \alpha_{j k}+i \sigma_{j k}=2 \operatorname{tr}\left[\left(R_{j}-m_{j}\right) \rho\left(R_{k}-m_{k}\right)\right] .
$$

The mean $m$ can be arbitrary, but the correlation matrix $\alpha$ must be real and symmetric and the positivity condition

$$
\alpha+i \sigma \geq 0
$$

must hold (this is an easy consequence of the canonical commutation relations $(3.57)$ ).

Our aim is now to distinguish exactly one state among all others with the same mean and correlation matrix. This is the point where the Weyl operators come into play. Each state $\rho \in \mathcal{S}(\mathcal{H})$ can be characterized uniquely by its quantum characteristic function $X \ni x \mapsto \operatorname{tr}[W(x) \rho] \in \mathbb{C}$ which should be regarded as the quantum Fourier transform of $\rho$ and is in fact the Fourier transform of the Wigner function of $\rho$ 165. We call $\rho$ Gaussian if

$$
\operatorname{tr}[W(x) \rho]=\exp \left(i m \cdot x-\frac{1}{4} x \cdot \alpha \cdot x\right)
$$

holds. By differentiation it is easy to check that $\rho$ has indeed mean $m$ and covariance matrix $\alpha$.

The most prominent examples for Gaussian states are the ground state $\rho_{0}$ of a system of $d$ harmonic oscillators (where the mean is 0 and $\alpha$ is given by the corresponding classical Hamiltonian) and its phase space translates $\rho_{m}=W(m) \rho W(-m)$ (with mean $m$ and the same $\alpha$ as $\rho_{0}$ ), which are known from quantum optics as coherent states. $\rho_{0}$ and $\rho_{m}$ are pure states and it can be shown that a Gaussian state is pure iff $\sigma^{-1} \alpha=-\mathbb{I I}$ holds (see [79], Ch. 5). Examples for mixed Gaussians are temperature states of harmonic oscillators. In one degree of freedom this is

$$
\rho_{N}=\frac{1}{N+1} \sum_{n=0}^{\infty}\left(\frac{N}{N+1}\right)^{n}|n\rangle\langle n|
$$

where $|n\rangle\langle n|$ denotes the number basis and $N$ is the mean photon number. The characteristic function of $\rho_{N}$ is

$$
\operatorname{tr}\left[W(x) \rho_{N}\right]=\exp \left[-\frac{1}{2}\left(N+\frac{1}{2}\right)|x|^{2}\right],
$$

and its correlation matrix is simply $\alpha=2(N+1 / 2) \mathbb{I}$

3.3.3. Entangled Gaussians. - Let us consider now bipartite systems. Hence the phase space $(V, \sigma)$ decomposes into a direct sum $V=V_{A} \oplus V_{B}$ (where $A$ stands for "Alice" and $B$ for "Bob") and the symplectic matrix $\sigma=\sigma_{A} \oplus \sigma_{B}$ is block diagonal with respect to this decomposition. If $W_{A}(x)$ respectively $W_{B}(y)$ denote Weyl operators, acting on the Hilbert spaces $\mathcal{H}_{A}, \mathcal{H}_{B}$, and corresponding to the phase spaces $V_{A}$ and $V_{B}$, it is easy to see that the tensor product $W_{A}(x) \otimes W_{B}(y)$ satisfies the Weyl relations with respect to $(V, \sigma)$. Hence by the Stone - von Neumann uniqueness theorem we can identify $W(x \oplus y), x \oplus y \in V_{a} \oplus V_{B}=V$ with $W_{A}(x) \otimes$ $W_{A}(y)$. This shows immediately that a state $\rho$ on $\mathcal{H}=\mathcal{H}_{A} \otimes \mathcal{H}_{B}$ is a product state iff its characteristic function factorizes. Separability is characterized as follows (we omit the proof, see [170] instead).

\footnotetext{
${ }^{5}$ n infinite dimensions we have to define separable states (in slight generalization to Definition 2.2 .5 as a trace-norm convergent convex sum of product states.
} 
TheOREM 3.3.1 A Gaussian state with covariance matrix $\alpha$ is separable iff there are covariance matrices $\alpha_{A}, \alpha_{B}$ such that

$$
\alpha \geq\left[\begin{array}{cc}
\alpha_{A} & 0 \\
0 & \alpha_{B}
\end{array}\right]
$$

holds.

This theorem is somewhat similar to Theorem 2.4.1: It provides a useful criterion as long as abstract considerations are concerned, but not for explicit calculations. In contrast to finite dimensional systems, however, separability of Gaussian states can be decided by an operational criterion in terms of nonlinear maps between matrices 65. To state it we have to introduce some terminology first. The key tool is a sequence of $2 n+2 m \times 2 n+2 m$ matrices $\alpha_{N}, N \in \mathbb{N}$, written in block matrix notation as

$$
\alpha_{N}=\left[\begin{array}{ll}
A_{N} & C_{N} \\
C_{N}^{T} & B_{N}
\end{array}\right]
$$

Given $\alpha_{0}$ the other $\alpha_{N}$ are recursively defined by:

$$
A_{N+1}=B_{N+1}=A_{N}-\operatorname{Re}\left(X_{N}\right) \text { and } C_{N+1}=-\operatorname{Im}\left(X_{N}\right)
$$

if $\alpha_{N}-i \sigma \geq 0$ and $\alpha_{N+1}=0$ otherwise. Here we have set $X_{N}=C_{N}\left(B_{N}-i \sigma_{B}\right)^{-1} C_{N}^{T}$ and the inverse denotes the pseudo inverse $]^{[0}$ if $B_{N}-i \sigma_{B}$ is not invertible. Now we can state the following theorem (see 65 for a proof):

THEOREM 3.3.2 Consider a Gaussian state $\rho$ of a bipartite system with correlation matrix $\alpha_{0}$ and the sequence $\alpha_{N}, N \in \mathbb{N}$ just defined.

1. If for some $N \in \mathbb{N}$ we have $A_{N}-i \sigma_{A} \nsupseteq 0$ then $\rho$ is not separable.

2. If there is on the other hand an $N \in \mathbb{N}$ such that $A_{N}-\left\|C_{N}\right\| \mathbb{I}-i \sigma_{A} \geq 0$, then the state $\rho$ is separable $\left(\left\|C_{N}\right\|\right.$ denotes the operator norm of $\left.C_{N}\right)$.

To check whether a Gaussian state $\rho$ is separable or not we have to iterate through the sequence $\alpha_{N}$ until either condition 1 or 2 holds. In the first case we know that $\rho$ is entangled and separable in the second. Hence only the question remains whether the whole procedure terminates after a finite number of iterations. This problem is treated in [65] and it turns out that the set of $\rho$ for which separability is decidable after a finite number of steps is the complement of a measure zero set (in the set of all separable states). Numerical calculations indicate in addition that the method converges usually very fast (typically less than five iterations).

To consider ppt states we first have to characterize the transpose for infinite dimensional systems. There are different ways to do that. We will use the fact that the adjoint of a matrix can be regarded as transposition followed by componentwise complex conjugation. Hence we define for any (possibly unbounded) operator $A^{T}=$ $C A^{*} C$, where $C: \mathcal{H} \rightarrow \mathcal{H}$ denotes complex conjugation of the wave function in position representation. This implies $Q_{j}^{T}=Q_{j}$ for position and $P_{j}^{T}=-P_{j}$ for momentum operators. If we insert the partial transpose of a bipartite state $\rho$ into Equation (3.61) we see that the correlation matrix $\widetilde{\alpha}_{j k}$ of $\rho^{T}$ picks up a minus sign whenever one of the indices belongs to one of Alice's momentum operators. To be a state $\widetilde{\alpha}$ should satisfy $\widetilde{\alpha}+i \sigma \geq 0$, but this is equivalent to $\alpha+i \widetilde{\sigma} \geq 0$, where in $\widetilde{\sigma}$ the corresponding components are reversed i.e. $\widetilde{\sigma}=\left(-\sigma_{A}\right) \oplus \sigma_{B}$. Hence we have shown

${ }^{6} A^{-1}$ is the pseudo inverse of a matrix $A$ if $A A^{-1}=A^{-1} A$ is the projector onto the range of $A$. If $A$ is invertible $A^{-1}$ is the usual inverse. 
Proposition 3.3.3 A Gaussian state is ppt iff its correlation matrix $\alpha$ satisfies

$$
\alpha+i \widetilde{\sigma} \geq 0 \text { with } \widetilde{\sigma}=\left[\begin{array}{cc}
-\sigma_{A} & 0 \\
0 & \sigma_{B}
\end{array}\right] \text {. }
$$

The interesting question is now whether the ppt criterion is for a given number of degrees of freedom equivalent to separability or not. The following theorem which was proved in 144 for $1 \times 1$ systems and in 170 in $1 \times d$ case gives a complete answer.

THEOREM 3.3.4 A Gaussian state of a quantum system with $1 \times d$ degrees of freedom (i.e. $\operatorname{dim} X_{A}=2$ and $\operatorname{dim} X_{B}=2 d$ ) is separable iff it is ppt; in other words iff the condition of Proposition 3.3.3 holds.

For other kinds of systems the ppt criterion may fail which means that there are entangled Gaussian states which are ppt. A systematic way to construct such states can be found in 170 . Roughly speaking, it is based on the idea to go to the boundary of the set of ppt covariance matrices, i.e. $\alpha$ has to satisfy Equation (3.62) and $(3.69)$ and it has to be a minimal matrix with this property. Using this method explicit examples for ppt and entagled Gaussians are constructed for $2 \times 2$ degrees of freedom (cf. 170 for details).

3.3.4. Gaussian channels. - Finally we want to give a short review on a special class of channels for infinite dimensional quantum systems (cf. 84 for details). To explain the basic idea note first that each finite set of Weyl operators $\left(W\left(x_{j}\right)\right.$, $j=1, \ldots, N, x_{j} \neq x_{k}$ for $j \neq k$ ) is linear independent. This can be checked easily using expectation values of $\sum_{j} \lambda_{j} W\left(x_{j}\right)$ in Gaussian states. Hence linear maps on the space of finite linear combinations of Weyl operators can be defined by $T[W(x)]=f(x) W(A x)$ where $f$ is a complex valued function on $V$ and $A$ is a $2 d \times 2 d$ matrix. If we choose $A$ and $f$ carefully enough, such that some continuity properties match $T$ can be extended in a unique way to a linear map on $\mathcal{B}(\mathcal{H})-$ which is, however, in general not completely positive.

This means we have to consider special choices for $A$ and $f$. The most easy case arises if $f \equiv 1$ and $A$ is a symplectic isomorphism, i.e. $A^{T} \sigma A=\sigma$. If this holds the map $V \ni x \mapsto W(A x)$ is a representation of the Weyl relations and therefore unitarily equivalent to the representation we have started with. In other words there is a unitary operator $U$ with $T[W(x)]=W(A x)=U W(x) U^{*}$, i.e. $T$ is unitarily implemented, hence completely positive and, in fact, well known as Bogolubov transformation.

If $A$ does not preserve the symplectic matrix, $f \equiv 1$ is no option. Instead we have to choose $f$ such that the matrices

$$
M_{j k}=f\left(x_{j}-x_{k}\right) \exp \left(-\frac{i}{2} x_{j} \cdot \sigma x_{k}+\frac{i}{2} A x_{j} \cdot \sigma A x_{k}\right)
$$

are positive. Complete positivity of the corresponding $T$ is then a standard result of abstract $\mathrm{C}^{*}$-algebra theory (cf. [51]). If the factor $f$ is in addition a Gaussian, i.e. $f(x)=\exp \left(-\frac{1}{2} x \cdot \beta x\right)$ for a positive definite matrix $\beta$ the cp-map $T$ is called a Gaussian channel.

A simple way to construct a Gaussian channel is in terms of an ancilla representation. More precisely, if $A: V \rightarrow V$ is an arbitrary linear map we can extend it to a symplectic map $V \ni x \mapsto A x \oplus A^{\prime} x \in V \oplus V^{\prime}$, where the symplectic vector space $\left(V^{\prime}, \sigma^{\prime}\right)$ now refers to the environment. Consider now the Weyl operator $W(x) \otimes W^{\prime}\left(x^{\prime}\right)=W\left(x, x^{\prime}\right)$ on the Hilbert space $\mathcal{H} \otimes \mathcal{H}^{\prime}$ associated to the phase space element $x \oplus x^{\prime} \in V \oplus V^{\prime}$. Since $A \oplus A^{\prime}$ is symplectic it admits a unitary Bogolubov transformation $U: \mathcal{H} \otimes \mathcal{H}^{\prime} \rightarrow \mathcal{H} \otimes \mathcal{H}^{\prime}$ with $U^{*} W\left(x, x^{\prime}\right) U=W\left(A x, A^{\prime} x\right)$. 
If $\rho^{\prime}$ denotes now a Gaussian density matrix on $\mathcal{H}^{\prime}$ describing the initial state of the environment we get a Gaussian channel by

$$
\operatorname{tr}\left[T^{*}(\rho) W(x)\right]=\operatorname{tr}\left[\rho \otimes \rho^{\prime} U^{*} W\left(x, x^{\prime}\right) U\right]=\operatorname{tr}[\rho W(A x)] \operatorname{tr}\left[\rho^{\prime} W\left(A^{\prime} x\right)\right] .
$$

Hence $T[W(x)]=f(x) W(A x)$ with $f(x)=\operatorname{tr}\left[\rho^{\prime} W\left(A^{\prime} x\right)\right]$.

Particular examples for Gaussian channels in the case of one degree of freedom are attenuation and amplification channels [81, 84]. They are given in terms of a real parameter $k \neq 1$ by $\mathbb{R}^{2} \ni x \mapsto A x=k x \in \mathbb{R}^{2}$

$$
\mathbb{R}^{2} \ni x \mapsto A^{\prime} x=\sqrt{1-k^{2}} x \in \mathbb{R}^{2}<1,
$$

for $k<1$ and

$$
\mathbb{R}^{2} \ni(q, p) \mapsto A^{\prime}(q, p)=(\kappa q,-\kappa p) \in \mathbb{R}^{2} \text { with } \kappa=\sqrt{k^{2}-1}
$$

for $k>1$. If the environment is initially in a thermal state $\rho_{\widetilde{N}}($ cf. Equation (3.64)) this leads to

$$
T[W(x)]=\exp \left[\frac{1}{2}\left(\frac{\left|k^{2}-1\right|}{2}+N_{c}\right) x^{2}\right] W(k x)
$$

where we have set $N_{c}=\left|k^{2}-1\right| \tilde{N}$. If we start initially with a thermal state $\rho_{N}$ it is mapped by $T$ again to a thermal state $\rho_{N^{\prime}}$ with mean photon number $N^{\prime}$ given by

$$
N^{\prime}=k^{2} N+\max \left\{0, k^{2}-1\right\}+N_{c} .
$$

If $N_{c}=0$ this means that $T$ amplifies $(k>1)$ or damps $(k<1)$ the mean photon number, while $N_{c}>0$ leads to additional classical, Gaussian noise. We will reconsider this channel in greater detail in Chapter 6 . 


\section{Chapter 4}

\section{Basic tasks}

After we have discussed the conceptual foundations of quantum information we will consider now some of its basic tasks. The spectrum ranges here from elementary processes, like teleportation 4.1 or error correction 4.4, which are building blocks for more complex applications, up to possible future technologies like quantum cryptography 4.6 and quantum computing 4.5 .

\subsection{Teleportation and dense coding}

Maybe the most striking feature of entanglement is the fact that otherwise impossible machines become possible if entangled states are used as an additional resource. The most prominent examples are teleportation and dense coding which we want to discuss in this section.

4.1.1. Impossible machines revisited: Classical teleportation. - We have already pointed out in the introduction that classical teleportation, i.e. transmission of quantum information over a classical information channel is impossible. With the material introduced in the last two chapters it is now possible to reconsider this subject in a slightly more mathematical way, which makes the following treatment of entanglement enhanced teleportation more transparent. To "teleport" the state $\rho \in$ $\mathcal{B}^{*}(\mathcal{H})$ Alice performs a measurement (described by a POV measure $E_{1}, \ldots, E_{N} \in$ $\mathcal{B}(\mathcal{H})$ ) on her system and gets a value $x \in X=\{1, \ldots, N\}$ with probability $p_{x}=$ $\operatorname{tr}\left(E_{x} \rho\right)$. These data she communicates to Bob and he prepares a $\mathcal{B}(\mathcal{H})$ system in the state $\rho_{x}$. Hence the overall state Bob gets if the experiment is repeated many times is: $\widetilde{\rho}=\sum_{x \in X} \operatorname{tr}\left(E_{x} \rho\right) \rho_{x}$ (cf. Figure 1.1). The latter can be rewritten as the composition

$$
\mathcal{B}^{*}(\mathcal{H}) \stackrel{E^{*}}{\longrightarrow} \mathcal{C}(X)^{*} \stackrel{D^{*}}{\longrightarrow} \mathcal{B}^{*}(\mathcal{H})^{*}
$$

of the channels

$$
\mathcal{C}(X) \ni f \mapsto E(f)=\sum_{x \in X} f(x) E_{x} \in \mathcal{B}(\mathcal{H})
$$

and

$$
\mathcal{C}^{*}(X) \ni p \mapsto D^{*}(p)=\sum_{x \in X} p_{x} \rho_{x} \in \mathcal{B}^{*}(\mathcal{H})
$$

i.e. $\widetilde{\rho}=D^{*} E^{*}(\rho)$ and this Equation makes sense even if $X$ is not finite. The teleportation is successful if the output state $\widetilde{\rho}$ can not be distinguished from the input state $\rho$ by any statistical experiment, i.e. if $D^{*} E^{*}(\rho)=\rho$. Hence the impossibility of classical teleportation can be rephrased simply as $E D \neq$ Id for all observables $E$ and all preparations $D$.

4.1.2. Entanglement enhanced teleportation. - Let us change our setup now slightly. Assume that Alice wants to send a quantum state $\rho \in \mathcal{B}^{*}(\mathcal{H})$ to Bob and that she shares an entangled state $\sigma \in \mathcal{B}^{*}(\mathcal{K} \otimes \mathcal{K})$ and an ideal classical communication channel $\mathrm{C}(X) \rightarrow \mathcal{C}(X)$ with him. Alice can perform a measurement $E: \mathcal{C}(X) \rightarrow \mathcal{B}(\mathcal{H} \otimes \mathcal{K})$ on the composite system $\mathcal{B}(\mathcal{H} \otimes \mathcal{K})$ consisting of the particle to teleport $(\mathcal{B}(\mathcal{H}))$ and her part of the entangled system $(\mathcal{B}(\mathcal{K}))$. Then she

communicates the classical data $x \in X$ to Bob and he operates with the parameter dependent operation $D: \mathcal{B}(\mathcal{H}) \rightarrow \mathcal{B}(\mathcal{K}) \otimes \mathcal{C}(X)$ appropriately on his particle (cf. 
Figure 4.1). Hence the overall procedure can be described by the channel $T=$ $(E \otimes \mathrm{Id}) D$, or in analogy to 4.1 )

$$
\mathcal{B}^{*}\left(\mathcal{H} \otimes \mathcal{K}^{\otimes 2}\right) \stackrel{E^{*} \otimes \mathrm{Id}}{\longrightarrow} \mathcal{C}^{*}(X) \otimes \mathcal{B}^{*}(\mathcal{K}) \stackrel{D^{*}}{\longrightarrow} \mathcal{B}^{*}(\mathcal{H}) .
$$

The teleportation of $\rho$ is successful if

$$
T^{*}(\rho \otimes \sigma):=D^{*}\left(\left(E^{*} \otimes \operatorname{Id}\right)(\rho \otimes \sigma)\right)=\rho
$$

holds, in other words if there is no statistical measurement which can distinguish the final state $T^{*}(\rho \otimes \sigma)$ of Bob's particle from the initial state $\rho$ of Alice's input system. The two channels $E$ and $D$ and the entangled state $\sigma$ form a teleportation scheme if Equation (4.5) holds for all states $\rho$ of the $\mathcal{B}(\mathcal{H})$ system, i.e. if each state of a $\mathcal{B}(\mathcal{H})$ system can be teleported without loss of quantum information.

Assume now that $\mathcal{H}=\mathcal{K}=\mathbb{C}^{d}$ and $X=\left\{0, \ldots, d^{2}-1\right\}$ holds. In this case we can define a teleportation scheme as follows: The entangled state shared by Alice and Bob is a maximally entangled state $\sigma=|\Omega\rangle\langle\Omega|$ and Alice performs a measurement which is given by the one dimensional projections $E_{j}=\left|\Phi_{j}\right\rangle\left\langle\Phi_{j}\right|$, where $\Phi_{j} \in \mathcal{H} \otimes \mathcal{H}, j=0, \ldots, d^{2}-1$ is a basis of maximally entangled vectors. If her result is $j=0, \ldots, d^{2}-1$ Bob has to apply the operation $\tau \mapsto U_{j}^{*} \tau U_{j}$ on his partner of the entangled pair, where the $U_{j} \in \mathcal{B}(\mathcal{H}), j=0, \ldots, d^{2}-1$ are an orthonormal family of unitary operators, i.e. $\operatorname{tr}\left(U_{j}^{*} U_{k}\right)=d \delta_{j k}$. Hence the parameter dependent operation $D$ has the form (in the Schrödinger picture):

$$
\mathcal{C}^{*}(X) \otimes \mathcal{B}^{*}(\mathcal{H}) \ni(p, \tau) \mapsto D^{*}(p, \tau)=\sum_{j=0}^{d^{2}-1} p_{j} U_{j}^{*} \tau U_{j} \in \mathcal{B}^{*}(\mathcal{H}) .
$$

Therefore we get for $T^{*}(\rho \otimes \sigma)$ from Equation 4.5)

$$
\begin{aligned}
\operatorname{tr}\left[T^{*}(\rho \otimes \sigma) A\right] & =\operatorname{tr}\left[(E \otimes \mathrm{Id})^{*}(\rho \otimes \sigma) D(A)\right] \\
& =\operatorname{tr}\left[\sum_{j=0}^{d^{2}-1} \operatorname{tr}_{12}\left[\left|\Phi_{j}\right\rangle\left\langle\Phi_{j}\right|(\rho \otimes \sigma)\right] U_{j}^{*} A U_{j}\right] \\
& =\sum_{j=0}^{d^{2}-1} \operatorname{tr}\left[(\rho \otimes \sigma)\left|\Phi_{j}\right\rangle\left\langle\Phi_{j}\right| \otimes\left(U_{j}^{*} A U_{j}\right)\right]
\end{aligned}
$$

here $\operatorname{tr}_{12}$ denotes the partial trace over the first two tensor factors (= Alice's qubits). If $\Omega$, the $\Phi_{j}$ and the $U_{j}$ are related by the equation

$$
\Phi_{j}=\left(U_{j} \otimes \mathbb{I}\right) \Omega
$$

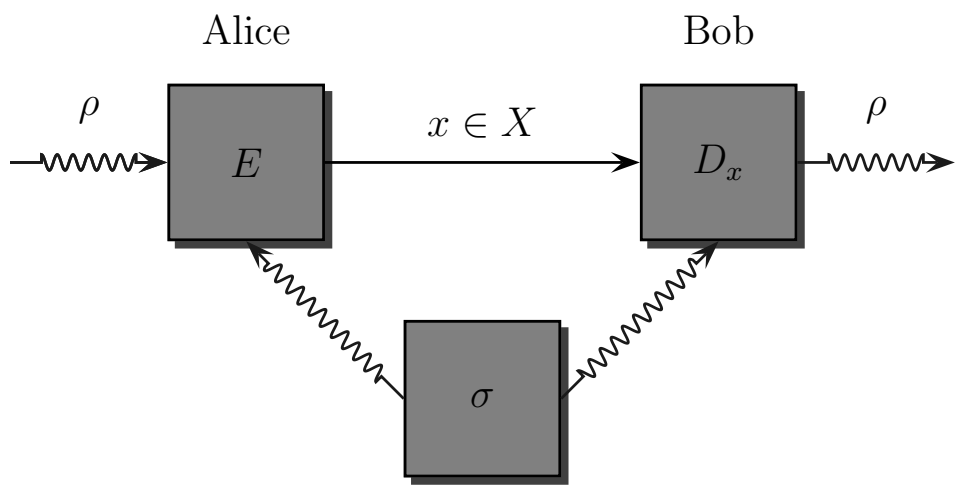

Figure 4.1: Entanglement enhanced teleportation 
it is a straightforward calculation to show that $T^{*}(\rho \otimes \sigma)=\rho$ holds as expected 168. If $d=2$ there is basically a unique choice: the $\Phi_{j}, j=0, \ldots, 3$ are the four Bell states (cf. Equation (3.3), $\Omega=\Phi_{0}$ and the $U_{j}$ are the identity and the three Pauli matrices. In this way we recover the standard example for teleportation, published for the first time in [11]. The first experimental realizations are [24, 22].

4.1.3. Dense coding. - We have just shown how quantum information can be transmitted via a classical channel, if entanglement is available as an additional resource. Now we are looking at the dual procedure: transmission of classical information over a quantum channel. To send the classical information $x \in X=\{1, \ldots, n\}$ to Bob, Alice can prepare a $d$-level quantum system in the state $\rho_{x} \in \mathcal{B}^{*}(\mathcal{H})$, sends it to Bob and he measures an observable given by positive operators $E_{1}, \ldots, E_{m}$. The probability for Bob to receive the signal $y \in X$ if Alice has sent $x \in X$ is $\operatorname{tr}\left(\rho_{x} E_{y}\right)$ and this defines a classical information channel by (cf. Subsection 3.2.3)

$$
\mathcal{C}^{*}(X) \ni p \mapsto\left(\sum_{x \in X} p(x) \operatorname{tr}\left(\rho_{x} E_{1}\right), \ldots, \sum_{x \in X} p(x) \operatorname{tr}\left(\rho_{x} E_{m}\right)\right) \in \mathcal{C}^{*}(X) .
$$

To get an ideal channel we just have to choose mutually orthogonal pure states $\rho_{x}=\left|\psi_{x}\right\rangle\left\langle\psi_{x}\right|, x=1, \ldots, d$ on Alice's side and the corresponding one-dimensional projections $E_{y}=\left|\psi_{y}\right\rangle\left\langle\psi_{y}\right|, y=1, \ldots, d$ on Bob's. If $d=2$ and $\mathcal{H}=\mathbb{C}^{2}$ it is possible to send one bit classical information via one qubit quantum information. The crucial point is now that the amount of classical information can be increased (doubled in the qubit case) if Alice shares an entangled state $\sigma \in \mathcal{S}(\mathcal{H} \otimes \mathcal{H})$ with Bob. To send the classical information $x \in X=\{1, \ldots, n\}$ to Bob, Alice operates on her particle with an operation $D_{x}: \mathcal{B}(\mathcal{H}) \rightarrow \mathcal{B}(\mathcal{H})$, sends it through an (ideal) quantum channel to Bob and he performs a measurement $E_{1}, \ldots, E_{n} \in \mathcal{B}(\mathcal{H} \otimes \mathcal{H})$ on both particles. The probability for Bob to measure $y \in X$ if Alice has send $x \in X$ is given by

$$
\operatorname{tr}\left[\left(D_{x} \otimes \mathrm{Id}\right)^{*}(\sigma) E_{y}\right],
$$

and this defines the transition matrix of a classical communication channel $T$. If $T$ is an ideal channel, i.e. if the transition matrix (4.12) is the identity, we will call $E$, $D$ and $\sigma$ a dense coding scheme (cf. Figure 4.2).

In analogy to Equation (4.4) we can rewrite the channel $T$ defined by (4.12) in terms of the composition

$$
\mathcal{C}^{*}(X) \otimes \mathcal{B}^{*}(\mathcal{H}) \otimes \mathcal{B}^{*}(\mathcal{H}) \stackrel{D^{*} \otimes \operatorname{Id}}{\longrightarrow} \mathcal{B}^{*}(\mathcal{H}) \otimes \mathcal{B}^{*}(\mathcal{H}) \stackrel{E^{*}}{\longrightarrow} \mathcal{C}^{*}(X)
$$

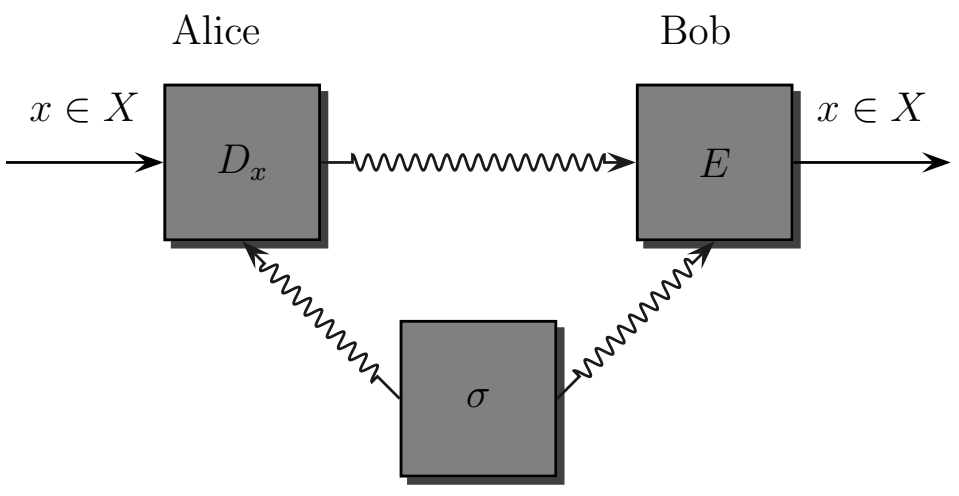

Figure 4.2: Dense coding 
of the parameter dependent operation

$$
D: \mathcal{C}^{*}(X) \otimes \mathcal{B}^{*}(\mathcal{H}) \rightarrow \mathcal{B}^{*}(\mathcal{H}), \quad p \otimes \tau \mapsto \sum_{j=1}^{n} p_{j} D_{j}(\tau)
$$

and the observable

$$
E: \mathcal{C}(X) \rightarrow \mathcal{B}(\mathcal{H} \otimes \mathcal{H}), \quad p \mapsto \sum_{j=1}^{n} p_{j} E_{j},
$$

i.e. $T^{*}(p)=E^{*} \circ\left(D^{*} \otimes \mathrm{Id}\right)(p \otimes \sigma)$. The advantage of this point of view is that it works as well for infinite dimensional Hilbert spaces and continuous observables.

Finally let us consider again the case where $\mathcal{H}=\mathbb{C}^{d}$ and $X=\left\{1, \ldots, d^{2}\right\}$. If we choose as in the last paragraph a maximally entangled vector $\Omega \in \mathcal{H} \otimes \mathcal{H}$, an orthonormal base $\Phi_{x} \in \mathcal{H} \otimes \mathcal{H}, j=x, \ldots, d^{2}$ of maximally entangled vectors and an orthonormal family $U_{x} \in \mathcal{B}(\mathcal{H} \otimes \mathcal{H}), x=1, \ldots, d^{2}$ of unitary operators, we can construct a dense coding scheme as follows: $E_{x}=\left|\Phi_{x}\right\rangle\left\langle\Phi_{x}\right|, D_{x}(A)=U_{x}^{*} A U_{x}$ and $\sigma=|\Omega\rangle\langle\Omega|$. If $\Omega$, the $\Phi_{x}$ and the $U_{x}$ are related by Equation (4.10) it is easy to see that we really get a dense coding scheme 168. If $d=2$ holds, we have to set again the Bell basis for the $\Phi_{x}, \Omega=\Phi_{0}$ and the identity and the Pauli matrices for the $U_{x}$. We recover in this case the standard example of dense coding proposed in 19] and we see that we can transfer two bits via one qubit, as stated above.

\subsection{Estimating and copying}

The impossibility of classical teleportation can be rephrased as follows: It is impossible to get complete information about the state $\rho$ of a quantum system by one measurement on one system. However, if we have many systems, say $N$, all prepared in the same state $\rho$ it should be possible to get (with a clever measuring strategy) as much information on $\rho$ as possible, provided $N$ is large enough. In this way we can circumvent the impossibility of devices like classical teleportation or quantum copying at least in an approximate way.

4.2.1. Quantum state estimation. - To discuss this idea in a more detailed way consider a number $N$ of $d$-level quantum systems, all of them prepared in the same (unknown) state $\rho \in \mathcal{B}^{*}(\mathcal{H})$. Our aim is to estimate the state $\rho$ by measurements on the compound system $\rho^{\otimes N}$. This is described in terms of an observable $E^{N}: \mathcal{C}\left(X_{N}\right) \rightarrow \mathcal{B}\left(\mathcal{H}^{\otimes N}\right)$ with values in a finite subset $X_{N} \subset \mathcal{S}(\mathcal{H})$ of the quantum state space $\mathcal{S}(\mathcal{H})$. According to Subsection 3.2.4 each such $E^{N}$ is given in terms of a tuple $E_{\sigma}^{N}, \sigma \in X_{N}$, by $E(f)=\sum_{\sigma} f(\sigma) E_{\sigma}^{N}$ hence we get for the expectation value of an $E_{N}$ measurement on systems in the state $\rho^{\otimes N}$ the density matrix $\widehat{\rho}_{N} \in \mathcal{S}(\mathcal{H})$ with matrix elements

$$
\left\langle\phi, \widehat{\rho}_{N} \psi\right\rangle=\sum_{x \in X_{N}}\langle\phi, \sigma \psi\rangle E_{\sigma}^{N} .
$$

We will call the channel $E^{N}$ an estimator and the criterion for a good estimator $E^{N}$ is that for any one-particle density operator $\rho$, the value measured on a state $\rho^{\otimes N}$ is likely to be close to $\rho$, i.e. that the probability

$$
K^{N}(\omega):=\operatorname{tr}\left(E^{N}(\omega) \rho^{\otimes N}\right) \text { with } E^{N}(\omega)=\sum_{\sigma \in X_{N} \cap \omega} E_{\sigma}^{N}
$$

\footnotetext{
${ }^{1}$ This is a severe restriction at this point and physically not very well motivated. There might be more general (i.e. continuous) observables taking their values in the whole state space $\mathcal{S}(\mathcal{H})$ which lead to much better estimates. However we do not discuss this possibility in order to keep mathematics more elementary.
} 
is small if $\omega \subset \mathcal{S}(\mathcal{H})$ is the complement of a small ball around $\rho$. Of course, we will look at this problem for large $N$. So the task is to find a whole sequence of observables $E^{N}, N=1,2, \ldots$, making error probabilities like (4.17) go to zero as $N \rightarrow \infty$.

The most direct way to get a family $E^{N}, N \in \mathbb{N}$ of estimators with this property is to perform a sequence of measurements on each of the $N$ input systems separately. A finite set of observables which leads to a successful estimation strategy is usually called a "quorum" (cf. e.g. 107, 162|). E.g. for $d=2$ we can perform alternating measurements of the three spin components. If $\rho=\frac{1}{2}(\mathbb{I}+\vec{x} \cdot \vec{\sigma})$ is the Bloch representation of $\rho$ (cf. Subsection 2.1.2) we see that the expectation values of these measurements are given by $\frac{1}{2}\left(1+x_{j}\right)$. Hence we get an arbitrarily good estimate if $N$ is large enough. A similar procedure is possible for arbitrary $d$ if we consider the generalized Bloch representation for $\rho$ (see again Subsection 2.1.2). There are however more efficient strategies based on "entangled" measurements (i.e. the $E_{N}(\sigma)$ can not be decomposed into pure tensor products) on the whole input system $\rho^{\otimes N}$ (e.g. [156, 99). Somewhat in between are "adaptive schemes" 663 consisting of separate measurements but the $j^{\text {th }}$ measurement depend on the results of $(j-1)^{\text {th }}$. We will reconsider this circle of questions in a more quantitative way in Chapter 0 .

4.2.2. Approximate cloning. - By virtue of the no-cloning theorem [173], it is impossible to produce $M$ perfect copies of a $d$-level quantum system if $N<M$ input systems in the common (unknown) state $\rho^{\otimes N}$ are given. More precisely there is no channel $T_{M N}: \mathcal{B}\left(\mathcal{H}^{\otimes M}\right) \rightarrow \mathcal{B}\left(\mathcal{H}^{\otimes N}\right)$ such that $T_{M N}^{*}\left(\rho^{\otimes N}\right)=\rho^{\otimes M}$ holds for all $\rho \in \mathcal{S}(\mathcal{H})$. Using state estimation, however, it is easy to find a device $T_{M N}$ which produces at least approximate copies which become exact in the limit $N, M \rightarrow \infty$ : If $\rho^{\otimes N}$ is given, we measure the observable $E^{N}$ and get the classical data $\sigma \in X_{N} \subset$ $\mathcal{S}(\mathcal{H})$, which we use subsequently to prepare $M$ systems in the state $\sigma^{\otimes M}$. In other words, $T_{M N}$ has the form

$$
\mathcal{B}^{*}\left(\mathcal{H}^{\otimes N}\right) \ni \tau \mapsto \sum_{\sigma \in X_{N}} \operatorname{tr}\left(E_{\sigma}^{N} \tau\right) \sigma^{\otimes M} \in \mathcal{B}^{*}\left(\mathcal{H}^{\otimes M}\right) .
$$

We see immediately that the probability to get wrong copies coincides exactly with the error probability of the estimator given in Equation (4.17). This shows first that we get exact copies in the limit $N \rightarrow \infty$ and second that the quality of the copies does not depend on the number $M$ of output systems, i.e. the asymptotic rate $\lim _{N, M \rightarrow \infty} M / N$ of output systems per input system can be arbitrary large.

The fact that we get classical data at an intermediate step allows a further generalization of this scheme. Instead of just preparing $M$ systems in the state $\sigma$ detected by the estimator, we can apply first an arbitrary transformation $F$ : $\mathcal{S}(\mathcal{H}) \rightarrow \mathcal{S}(\mathcal{H})$ on the density matrix $\sigma$ and prepare $F(\sigma)^{\otimes M}$ instead of $\sigma^{\otimes M}$. In this way we get the channel (cf. Figure 4.3)

$$
\mathcal{B}^{*}\left(\mathcal{H}^{\otimes N}\right) \ni \tau \mapsto \sum_{\sigma \in X_{N}} \operatorname{tr}\left(E_{\sigma}^{N} \tau\right) F(\sigma)^{\otimes M} \in \mathcal{B}^{*}\left(\mathcal{H}^{\otimes M}\right),
$$

i.e. a physically realizable device which approximates the impossible machine $F$. The probability to get a bad approximation of the state $F(\rho)^{\otimes M}$ (if the input state was $\rho^{\otimes N}$ ) is again given by the error probability of the estimator and we get a perfect realization of $F$ at arbitrary rate as $M, N \rightarrow \infty$.

There are in particular two interesting tasks which become possible this way: The first is the "universal not gate" which associates to each pure state of a qubit the unique pure state orthogonal to it 36]. This is a special example of a antiunitarily implemented symmetry operation and therefore not completely positive. The second example is the purification of states [46, 100]. Here it is assumed that the input 


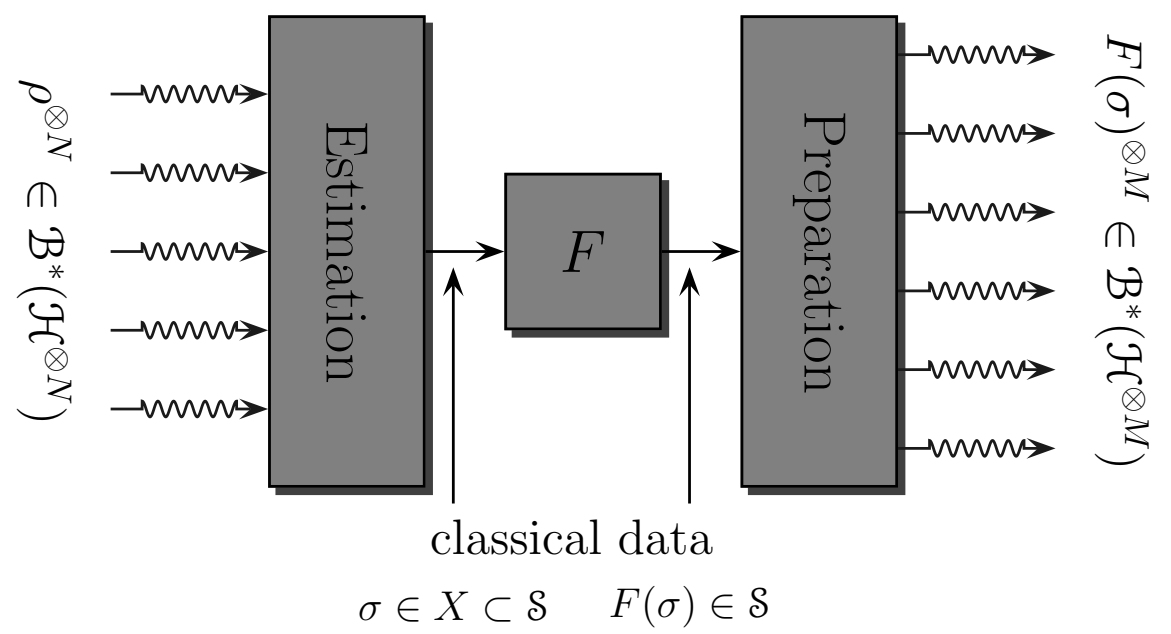

Figure 4.3: Approximating the impossible machine $F$ by state estimation.

states were once pure but have passed later on a depolarizing channel $|\phi\rangle\langle\phi| \mapsto$ $\vartheta|\phi\rangle\langle\phi|+(1-\vartheta) \mathbb{I} / d$. If $\vartheta>0$ this map is invertible but its inverse does not describe an allowed quantum operation because it maps some density operators to operators with negative eigenvalues. Hence the reversal of noise is not possible with a one shot operation but can be done with high accuracy if enough input systems are available. We rediscuss this topic in Chapter 7 .

\subsection{Distillation of entanglement}

Let us return now to entanglement. We have seen in Section 4.1 that maximally entangled states play a crucial role for processes like teleportation and dense coding. In practice however entanglement is a rather fragile property: If Alice produces a pair of particles in a maximally entangled state $|\Omega\rangle\langle\Omega| \in \mathcal{S}\left(\mathcal{H}_{A} \otimes \mathcal{H}_{B}\right)$ and distributes one of them over a great distance to Bob, both end up with a mixed state $\rho$ which contains much less entanglement then the original and which can not be used any longer for teleportation. The latter can be seen quite easily if we try to apply the qubit teleportation scheme (Subsection 4.1.2) with a non-maximally entangled isotropic state (Equation (3.15) with $\lambda>0$ ) instead of $\Omega$.

Hence the question arises, whether it is possible to recover $|\Omega\rangle\langle\Omega|$ from $\rho$, or, following the reasoning from the last section, at least a small number of (almost) maximally entangled states from a large number $N$ of copies of $\rho$. However since the distance between Alice and Bob is big (and quantum communication therefore impossible) only LOCC operations (Section 3.2.6) are available for this task (Alice and Bob can only operate on their respective particles, drop some of them and communicate classically with one another). This excludes procedures like the purification scheme just sketched, because we would need "entangled" measurements to get an asymptotically exact estimate for the state $\rho$. Hence we need a sequence of LOCC channels

$$
T_{N}: \mathcal{B}\left(\mathbb{C}^{d_{N}} \otimes \mathbb{C}^{d_{N}}\right) \rightarrow \mathcal{B}\left(\mathcal{H}_{A}^{\otimes N} \otimes \mathcal{H}_{B}^{\otimes N}\right)
$$

such that

$$
\| T_{N}^{*}\left(\rho^{\otimes N}\right)-\left|\Omega_{N}\right\rangle\left\langle\Omega_{N}\right| \|_{1} \rightarrow 0, \text { for } N \rightarrow \infty
$$

holds, with a sequence of maximally entangled vectors $\Omega_{N} \in \mathbb{C}^{d_{N}} \otimes \mathbb{C}^{d_{N}}$. Note that we have to use here the natural isomorphism $\mathcal{H}_{A}^{\otimes N} \otimes \mathcal{H}_{B}^{\otimes N} \cong\left(\mathcal{H}_{A} \otimes \mathcal{H}_{B}\right)^{\otimes N}$, i.e. we 
have to reshuffle $\rho^{\otimes N}$ such that the first $N$ tensor factors belong to Alice $\left(\mathcal{H}_{A}\right)$ and the last $N$ to Bob $\left(\mathcal{H}_{B}\right)$. If confusion can be avoided we will use this isomorphism in the following without a further note. We will call a sequence of LOCC channels, $T_{N}$ satisfying (4.21) with a state $\rho \in \mathcal{S}\left(\mathcal{H}_{A} \otimes \mathcal{H}_{B}\right)$ a distillation scheme for $\rho$ and $\rho$ is called distillable if it admits a distillation scheme. The asymptotic rate with which maximally entangled states can be distilled with a given protocol is

$$
\liminf _{n \rightarrow \infty} \log _{2}\left(d_{N}\right) / N \text {. }
$$

This quantity will become relevant in the framework of entanglement measures (Chapter 5).

4.3.1. Distillation of pairs of qubits. - Concrete distillation protocols are in general rather complicated procedures. We will sketch in the following how any pair of entangled qubits can be distilled. The first step is a scheme proposed for the first time by Bennett et. al. [12]. It can be applied if the maximally entangled fraction $\mathcal{F}$ (Equation (3.4)) is greater than 1/2. As indicated above, we assume that Alice and Bob share a large amount of pairs in the state $\rho$, so that the total state is $\rho^{\otimes N}$. To obtain a smaller number of pairs with a higher $\mathcal{F}$ they proceed as follows:

1. First they take two pairs (let us call them pair 1 and pair 2), i.e. $\rho \otimes \rho$ and apply to each of them the twirl operation $P_{\mathrm{UU}}$ associated to isotropic states (cf. Equation (3.18)). This can be done by LOCC operations in the following way: Alice selects at random (respecting the Haar measure on $\mathrm{U}(2)$ ) a unitary operator $U$ applies it to her qubits and sends to Bob which transformation she has chosen; then he applies $\bar{U}$ to his particles. They end up with two isotropic states $\widetilde{\rho} \otimes \widetilde{\rho}$ with the same maximally entangled fraction as $\rho$.

2. Each party performs the unitary transformation

$$
U_{\mathrm{XOR}}:|a\rangle \otimes|b\rangle \mapsto|a\rangle \otimes|a+b \quad \bmod 2\rangle
$$

on his/her members of the pairs.

3. Finally Alice and Bob perform locally a measurement in the basis $|0\rangle,|1\rangle$ on pair 1 and discards it afterwards. If the measurements agree, pair 2 is kept and has a higher $\mathcal{F}$. Otherwise pair 2 is discarded as well.

If this procedure is repeated over and over again, it is possible to get states with an arbitrarily high $\mathcal{F}$, but we have to sacrifice more and more pairs and the asymptotic rate is zero. To overcome this problem we can apply the scheme above until $\mathcal{F}(\rho)$ is high enough such that $1+\operatorname{tr}(\rho \ln \rho) \geq 0$ holds and then we continue with another scheme called hashing [16] which leads to a nonvanishing rate.

If finally $\mathcal{F}(\rho) \leq 1 / 2$ but $\rho$ is entangled, Alice and Bob can increase $\mathcal{F}$ for some of their particles by filtering operations [9, 67]. The basic idea is that Alice applies an instrument $T: \mathcal{C}(X) \otimes \mathcal{B}(\mathcal{H}) \rightarrow \mathcal{B}(\mathcal{H})$ with two possible outcomes $(X=\{1,2\})$ to her particles. Hence the state becomes $\rho \mapsto p_{x}^{-1}\left(T_{x} \otimes \mathrm{Id}\right)^{*}(\rho), x=1,2$ with probability $p_{x}=\operatorname{tr}\left[T_{x}^{*}(\rho)\right]$ (cf. Subsection 3.2.5 in particular Equation (3.50) for the definition of $\left.T_{x}\right)$. Alice communicates her measuring result $x$ to Bob and if $x=1$ they keep the particle otherwise $(x=2)$ they discard it. If the instrument $T$ was correctly chosen Alice and Bob end up with a state $\widetilde{\rho}$ with higher maximally entangled fraction. To find an appropriate $T$ note first that there are $\psi \in \mathcal{H} \otimes \mathcal{H}$ with $\langle\psi,(\operatorname{Id} \otimes \Theta) \rho \psi\rangle \leq 0$ (this follows from Theorem 2.4.3 since $\rho$ is by assumption entangled) and second that we can write each vector $\psi \in \mathcal{H} \otimes \mathcal{H}$ as $\left(X_{\psi} \otimes \mathbb{I}\right) \Phi_{0}$ with the Bell state $\Phi_{0}$ and an appropriately chosen operator $X_{\psi}$ (see Subsection 3.1.1). 
Now we can define $T$ in terms of the two operations $T_{1}, T_{2}$ (cf. Equation (3.52)) with

$$
T_{1}(A)=X_{\psi}^{*} A X_{\psi}^{-1}, \quad \mathrm{Id}-T_{1}=T_{2}
$$

It is straightforward to check that we end up with

$$
\widetilde{\rho}=\frac{\left(T_{x} \otimes \mathrm{Id}\right)^{*}(\rho)}{\operatorname{tr}\left[\left(T_{x} \otimes \mathrm{Id}\right)^{*}(\rho)\right]}
$$

such that $\mathcal{F}(\widetilde{\rho})>1 / 2$ holds and we can continue with the scheme described in the previous paragraph.

4.3.2. Distillation of isotropic states. - Consider now an entangled isotropic state $\rho$ in $d$ dimensions, i.e. we have $\mathcal{H}=\mathbb{C}^{d}$ and $0 \leq \operatorname{tr}(\widetilde{F} \rho) \leq 1$ (with the operator $\widetilde{F}$ of Subsection 3.1.3). Each such state is distillable via the following scheme [27, 85]: First Alice and Bob apply a filter operation $T: \mathcal{C}(X) \otimes \mathcal{B}(\mathcal{H}) \rightarrow \mathcal{B}(\mathcal{H})$ on their respective particle given by $T_{1}(A)=P A P, T_{2}=1-T_{1}$ where $P$ is the projection onto a two dimensional subspace. If both measure the value 1 they get a qubit pair in the state $\widetilde{\rho}=\left(T_{1} \otimes T_{1}\right)(\rho)$. Otherwise they discard their particles (this requires classical communication). Obviously the state $\widetilde{\rho}$ is entangled (this is easily checked) hence they can proceed as in the previous Subsection.

The scheme just proposed can be used to show that each state $\rho$ which violates the reduction criterion (cf. Subsection 2.4.3) can be distilled 857. The basic idea is to project $\rho$ with the twirl $P_{\mathrm{UU}}$ (which is LOCC as we have seen above; cf. Subsection 4.3.1) to an isotropic state $P_{\mathrm{UU}}(\rho)$ and to apply afterwards the procedure from the last paragraph. We only have to guarantee that $P_{\mathrm{UU}}(\rho)$ is entangled. To this end use a vector $\psi \in \mathcal{H} \otimes \mathcal{H}$ with $\left\langle\psi,\left(\mathbb{I} \otimes \operatorname{tr}_{1}(\rho)-\rho\right) \psi\right\rangle<0$ (which exists by assumption since $\rho$ violates the reduction criterion) and to apply the filter operation given by $\psi$ via Equation (4.24).

4.3.3. Bound entangled states. - It is obvious that separable states are not distillable, because a LOCC operation map separable states to separable states. However is each entangled state distillable? The answer, maybe somewhat surprising, is no and an entangled state which is not distillable is called bound entangled 87] (distillable states are sometimes called free entangled, in analogy to thermodynamics). Examples of bound entangled states are all ppt entangled states [87: This is an easy consequence of the fact that each separable channel (and therefore each LOCC channel as well) maps ppt states to ppt states (this is easy to check), but a maximally entangled state is never ppt. It is not yet known, whether bound entagled npt states exists, however, there are at least some partial results: 1 . It is sufficient to solve this question for Werner states, i.e. if we can show that each npt Werner state is distillable it follows that all npt states are distillable 85. 2. Each npt Gaussian state is distllable [64]. 3. For each $N \in \mathbb{N}$ there is an npt Werner state $\rho$ which is not " $N$-copy distillable", i.e. $\left\langle\psi, \rho^{\otimes N} \psi\right\rangle \geq 0$ holds for each pure state $\psi$ with exactly two Schmidt summands [55, 58]. This gives some evidence for the existence of bound entangled npt states because $\rho$ is distillabile iff it is $N$-copy distillability for some $N$ [87, 55, 58].

Since bound entangled states can not be distilled, they can not be used for teleportation. Nevertheless bound entanglement can produce a non-classical effect, called "activation of bound entanglement" [92]. To explain the basic idea, assume that Alice and Bob share one pair of particles in a distillable state $\rho_{f}$ and many particles in a bound entangled state $\rho_{b}$. Assume in addition that $\rho_{f}$ can not be used for teleportation, or, in other words if $\rho_{f}$ is used for teleportation the particle Bob receives is in a state $\sigma^{\prime}$ which differs from the state $\sigma$ Alice has send. This problem can not be solved by distillation, since Alice and Bob share only one pair 
of particles in the state $\rho_{f}$. Nevertheless they can try to apply an appropriate filter operation on $\rho$ to get with a certain probability a new state which leads to a better quality of the teleportation (or, if the filtering fails, to get nothing at all). It can be shown however [88] that there are states $\rho_{f}$ such that the error occuring in this process (e.g. measured by the trace norm distance of $\sigma$ and $\sigma^{\prime}$ ) is always above a certain threshold. This is the point where the bound entangled states $\rho_{b}$ come into play: If Alice and Bob operate with an appropriate protocol on $\rho_{f}$ and many copies of $\rho_{b}$ the distance between $\sigma$ and $\sigma^{\prime}$ can be made arbitrarily small (although the probability to be successful goes to zero). Another example for an activation of bound entanglement is related to distillability of npt states: If Alice and Bob share a certain ppt-entangled state as additional recource each npt state $\rho$ becomes distillable (evem if $\rho$ is bound entangled) 60, 104. For a more detailed survey of the role of bound entanglement and further references see [91].

\subsection{Quantum error correction}

If we try to distribute quantum information over large distances or store it for a long time in some sort of "quantum memory" we always have to deal with "decoherence effects", i.e. unavoidable interactions with the environment. This results in a significant information loss, which is particularly bad for the functioning of a quantum computer. Similar problems arise as well in a classical computer, but the methods used there to circumvent the problems can not be transferred to the quantum regime. E.g. the most simple strategy to protect classical information against noise is redundancy: instead of storing the information once we make three copies and decide during readout by a majority vote which bit to take. It is easy to see that this reduces the probability of an error from order $\epsilon$ to $\epsilon^{2}$. Quantum mechanically however such a procedure is forbidden by the no cloning theorem.

Nevertheless quantum error correction is possible although we have to do it in a more subtle way than just copying; this was observed for the first time independently in [39] and [146]. Let us consider first the general scheme and assume that $T$ : $\mathcal{B}(\mathcal{K}) \rightarrow \mathcal{B}(\mathcal{K})$ is a noisy quantum channel. To send quantum systems of type $\mathcal{B}(\mathcal{H})$ undisturbed through $T$ we need an encoding channel $E: \mathcal{B}(\mathcal{K}) \rightarrow \mathcal{B}(\mathcal{H})$ and a decoding channel $D: \mathcal{B}(\mathcal{H}) \rightarrow \mathcal{B}(\mathcal{K})$ such that $E T D=$ Id holds, respectively $D^{*} T^{*} E^{*}=$ Id in the Schrödinger picture; cf. Figure 4.4.

A powerful error correction scheme should not be restricted to one particular type of error, i.e. one particular noisy channel $T$. Assume instead that $\mathfrak{E} \subset \mathcal{B}(\mathcal{K})$ is

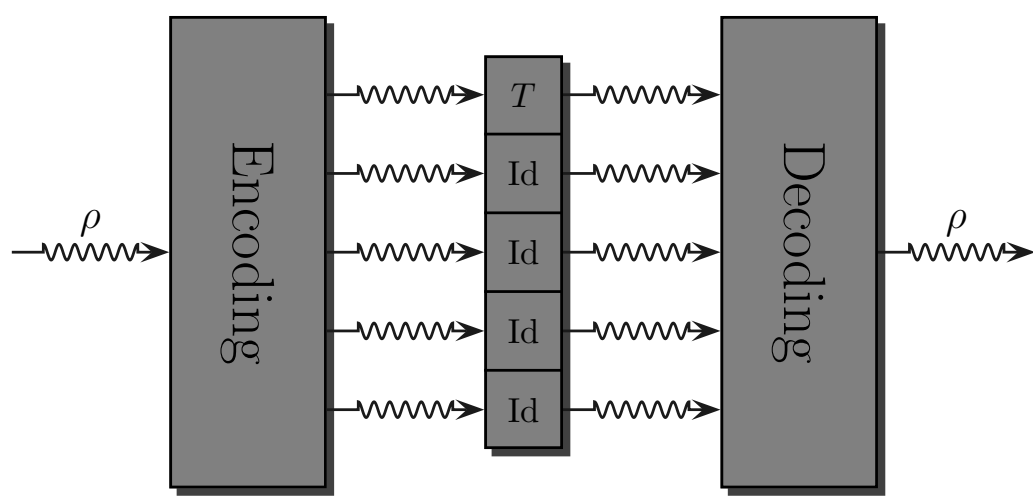

Figure 4.4: Five bit quantum code: Encoding one qubit into five and correcting one error. 
a linear subspace of "error operators" and $T$ is any channel given by

$$
T_{*}(\rho)=\sum_{j} F_{j} \rho F_{j}^{*}, \quad F_{j} \in \mathfrak{E} .
$$

An isometry $V: \mathcal{H} \rightarrow \mathcal{K}$ is called an error correcting code for $\mathfrak{E}$ if for each $T$ of the form (4.26) there is a decoding channel $D: \mathcal{B}(\mathcal{H}) \rightarrow \mathcal{B}(\mathcal{K})$ with $D_{*}\left(T\left(V \rho V^{*}\right)\right)=\rho$ for all $\rho \in \mathcal{S}(\mathcal{H})$. By the theory of Knill and Laflamme 103 this is equivalent to the factorization condition

$$
\left\langle V \psi, F_{j}^{*} F_{k} V \phi\right\rangle=\omega\left(F_{j}^{*} F_{k}\right)\langle\psi, \phi\rangle
$$

where $\omega\left(F_{j}^{*} F_{k}\right)$ is a factor which does not depend on the arbitrary vectors $\psi, \phi \in \mathcal{H}$.

The most relevant examples of error correcting codes are those which generalize the classical idea of sending multiple copies in a certain sense. This means we encode a small number $N$ of $d$-level systems into a big number $M \gg N$ of systems of the same type, which are then transmitted and decoded back into $N$ systems afterwards. During the transmission $K<M$ arbitrary errors are allowed. Hence we have $\mathcal{H}=\mathcal{H}_{1}^{\otimes N}, \mathcal{K}=\mathcal{H}_{1}^{\otimes M}$ with $\mathcal{H}_{1}=\mathbb{C}^{d}$ and $T$ is an arbitrary tensor product of $K$ noisy channels $S_{j}, j=1, \ldots, K$ and $M-K$ ideal channels Id. The most well known code for this type of error is the "five-bit code" where one qubit is encoded into five and one error is corrected [16] (cf. Figure 4.4 for $N=1, M=5$ and $K=1$ ). To define the corresponding error space $\mathfrak{E}$ consider the finite sets $X=\{1, \ldots, N\}$ and $Y=\{1+N, \ldots, M+N\}$ and define first for each subset $Z \subset Y$ :

$$
\begin{aligned}
\mathfrak{E}(Z)=\operatorname{span}\left\{A_{1} \otimes \cdots \otimes A_{M} \in \mathcal{B}(\mathcal{K}) \mid\right. \\
\left.\qquad A_{j} \in \mathcal{B}\left(\mathcal{H}_{1}\right) \text { arbitrary for } j+N \in Z, A_{j}=\mathbb{I} \text { otherwise }\right\} .
\end{aligned}
$$

$\mathfrak{E}$ is now the span of all $\mathfrak{E}(Z)$ with $|Z| \leq K$ (i.e. the length of $Z$ is less or equal to $K)$. We say that an error correcting code for this particular $\mathfrak{E}$ corrects $K$ errors.

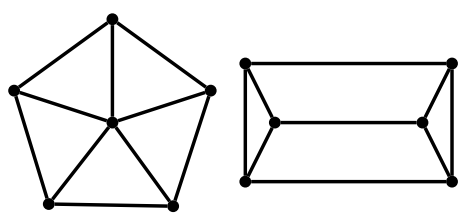

Figure 4.5: Two graphs belonging to (equivalent) five bit codes. The input node can be chosen in both cases arbitrarily.

vertices, labeled by $X$ and a set of output vertices labeled by $Y$. The links of the graph are given by the adjacency matrix, i.e. a $N+M \times N+M$ matrix $\Gamma$ with $\Gamma_{j k}=1$ if node $k$ and $j$ are linked and $\Gamma_{j k}=0$ otherwise. With respect to $\Gamma$ we can define now an isometry $V_{\Gamma}: \mathcal{H}_{1}^{\otimes N} \rightarrow \mathcal{H}_{1}^{\otimes M}$ by

$$
\left\langle j_{N+1} \ldots j_{N+M}\left|V_{\Gamma}\right| j_{1} \ldots j_{N}\right\rangle=\exp \left(\frac{i \pi}{d} \vec{j} \cdot \Gamma \vec{j}\right)
$$

with $\vec{j}=\left(j_{1}, \ldots, j_{N+M}\right) \in \mathbb{Z}_{d}^{N+M}$ (where $\mathbb{Z}_{d}$ denotes the cyclic group with $d$ elements). There is an easy condition under which $V_{\Gamma}$ is an error correcting code. To write it down we need the following additional terminology: We say that an error correcting code $V: \mathcal{H}_{1}^{\otimes N} \rightarrow \mathcal{H}_{1}^{\otimes M}$ detects the error configuration $Z \subset Y$ if

$$
\langle V \psi, F V \phi\rangle=\omega(F)\langle\psi, \phi\rangle \quad \forall F \in \mathfrak{E}(Z)
$$

holds. With Equation (4.27) it is easy to see that $V$ corrects $K$ errors iff it detects all error configurations of length $2 K$ or less. Now we have the following theorem: 
THEOREM 4.4.1 The quantum code $V_{\Gamma}$ defined in Equation (4.29) detects the error configuration $Z \subset Y$ if the system of equations

$$
\sum_{l \in X \cup Z} \Gamma_{k l} g_{l}=0, \quad k \in Y \backslash E, g_{l} \in \mathbb{Z}_{d}
$$

implies that

$$
g_{l}=0, l \in X \text { and } \sum_{l \in Z} \Gamma_{k l} g_{l}=0, k \in X
$$

holds.

We omit the proof, see [137] instead. Two particular examples (which are equivalent!) are given in Figure 4.5. In both cases we have $N=1, M=5$ and $K=1$ i.e. one input node, which can be chosen arbitrarily, five output nodes and the corresponding codes correct one error. For a more detailed survey on quantum error correction, in particular for more examples we refer to [20].

\subsection{Quantum computing}

Quantum computing is without a doubt the most prominent and most far reaching application of quantum information theory, since it promises on the one hand, "exponential speedup" for some problems which are "hard to solve" with a classical computer, and gives completely new insights into classical computing and complexity theory on the other. Unfortunately, an exhaustive discussion would require its own review article. Hence we we are only able to give a short overview (see Part II of [122] for a more complete presentation and for further references).

4.5.1. The network model of classical computing. - Let us start with a brief (and very informal) introduction to classical computing (for a more complete review and hints for further reading see Chapter 3 of [122]). What we need first is a mathematical model for computation. There are in fact several different choices and the Turing machine 152 is the most prominent one. More appropriate for our purposes is, however, the so called network model, since it allows an easier generalization to the quantum case. The basic idea is to interpret a classical (deterministic) computation as the evaluation of a map $f: \mathbb{B}^{N} \rightarrow \mathbb{B}^{M}$ (where $\mathbb{B}=\{0,1\}$ denotes the field with two elements) which maps $N$ input bits to $M$ output bits. If $M=1$ holds $f$ is called a boolean function and it is for many purposes sufficient to consider this special case - each general $f$ is in fact a Cartesian product of boolean functions.

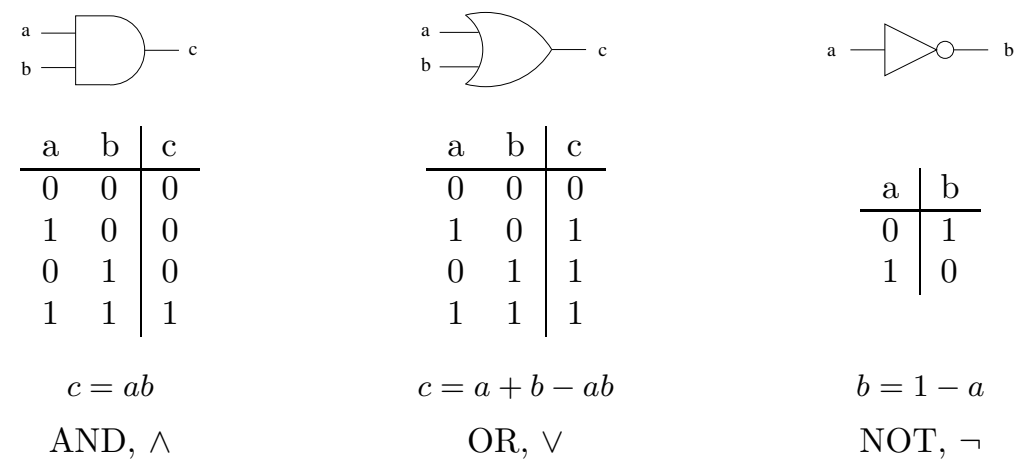

Figure 4.6: Symbols and definition for the three elementary gates AND, OR and NOT. 
Particular examples are the three elementary gates AND, OR and NOT defined in Figure 4.6 and arbitrary algebraic expressions constructed from them: e.g. the XOR gate $(x, y) \mapsto x+y \bmod 2$ which can be written as $(x \vee y) \wedge \neg(x \wedge y)$. It is now a standard result of boolean algebra that each boolean function can be represented in this way and there are in general many possibilities to do this. A special case is the disjunctive normal form of $f$; cf 161. To write such an expression down in form of equations is, however, somewhat confusing. $f$ is therefore expressed most conveniently in graphical form as a circuit or network, i.e. a graph $C$ with nodes representing elementary gates and edges ("wires") which determine how the gates should be composed; cf. Figure 4.7 for an example. A classical computation can now be defined as a circuit applied to a specified string of input bits.

Variants of this model arise if we replace AND, OR and NOT by another (finite) set $G$ of elementary gates. We only have to guarantee that each function $f$ can be expressed as a composition of elements from $G$. A typical example for $G$ is the set which contains only the NAND gate $(x, y) \mapsto x \uparrow y=\neg(x \wedge y)$. Since AND, OR and NOT can be rewritten in terms of NAND (e.g. $\neg x=x \uparrow x$ ) we can calculate each boolean function by a circuit of NAND gates.

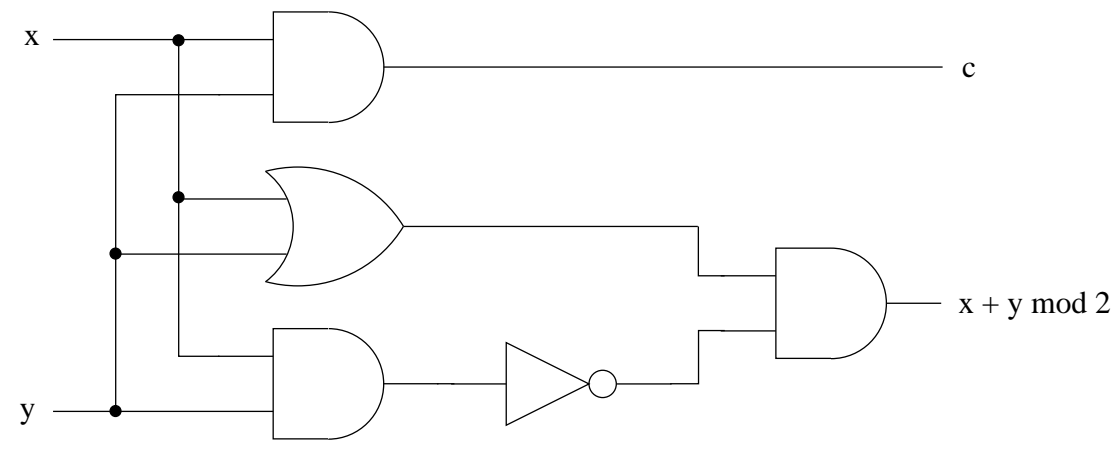

Figure 4.7: Half-adder circuit as an example for a boolean network.

4.5.2. Computational complexity. - One of the most relevant questions within classical computing, and the central subject of computational complexity, is whether a given problem is easy to solve or not, where "easy" is defined in terms of the scaling behavior of the resources needed in dependence of the size of the input data. We will give in the following a rough survey over the most basic aspects of this field, while we refer the reader to [124] for a detailed presentation.

To start with, let us specify the basic question in greater detail. First of all the problems we want to analyze are decision problems which only give the two possible values "yes" and "no". They are mathematically described by boolean functions acting on bit strings of arbitrary size. A well known example is the factoring problem given by the function fac with fac $(m, l)=1$ if $m$ (more precisely the natural number represented by $m$ ) has a divisor less then $l$ and $\operatorname{fac}(m, l)=0$ otherwise. Note that many tasks of classical computation can be reformulated this way, so that we do not get a severe loss of generality. The second crucial point we have to clarify is the question what exactly are the resources we have mentioned above and how we have to quantify them. A natural physical quantity which come into mind immediately is the time needed to perform the computation (space is another candidate, which we do not discuss here, however). Hence the question we have to discuss is how the computation time $t$ depends on the size $L$ of the input data $x$ (i.e. the length $L$ of the smallest register needed to represent $x$ as a bit string).

However a precise definition of "computation time" is still model dependent. For a Turing machine we can take simply the number of head movements needed to 
solve the problem, and in the network model we choose the number of steps needed to execute the whole circuit, if gates which operate on different bits are allowed to work simultaneously 2 Even with a fixed type of model the functional behavior of $t$ depends on the set of elementary operations we choose, e.g. the set of elementary gates in the network model. It is therefore useful to divide computational problems into complexity classes whose definitions do not suffer under model dependent aspects. The most fundamental one is the class $\mathbf{P}$ which contains all problems which can be computed in "polynomial time", i.e. $t$ is, as a function of $L$, bounded from above by a polynomial. The model independence of this class is basically the content of the strong Church Turing hypotheses which states, roughly speaking, that each model of computation can be simulated in polynomial time on a probabilistic Turing machine.

Problems of class $\mathbf{P}$ are considered "easy", everything else is "hard". However even if a (decision) problem is hard the situation is not hopeless. E.g. consider the factoring problem fac described above. It is generally believed (although not proved) that this problem is is not in class $\mathbf{P}$. But if somebody gives us a divisor $p<l$ of $m$ it is easy to check whether $p$ is really a factor, and if the answer is true we have computed $\operatorname{fac}(m, l)$. This example motivates the following definition: A decision problem $f$ is in class NP ("nondeterministic polynomial time") if there is a boolean function $f^{\prime}$ in class $\mathbf{P}$ such that $f^{\prime}(x, y)=1$ for some $y$ implies $f(x)$. In our example fac ${ }^{\prime}$ is obviously defined by $\operatorname{fac}^{\prime}(m, l, p)=1 \Leftrightarrow p<l$ and $p$ is a devisor of $m$. It is obvious that $\mathbf{P}$ is a subset of $\mathbf{N P}$ the other inclusion however is rather nontrivial. The conjecture is that $\mathbf{P} \neq \mathbf{N P}$ holds and great parts of complexity theory are based on it. Its proof (or disproof) however represents one of the biggest open questions of theoretical informatics.

To introduce a third complexity class we have to generalize our point of view slightly. Instead of a function $f: \mathbb{B}^{N} \rightarrow \mathbb{B}^{M}$ we can look at a noisy classical $T$ which sends the input value $x \in \mathbb{B}^{N}$ to a probability distribution $T_{x y}, y \in \mathbb{B}^{M}$ on $\mathbb{B}^{M}$ (i.e. $T_{x y}$ is the transition matrix of the classical channel $T$; cf. Subsection 3.2.3). Roughly speaking, we can interpret such a channel as a probabilistic computation which can be realized as a circuit consisting of "probabilistic gates". This means there are several different ways to proceed at each step and we use a classical random number generator to decide which of them we have to choose. If we run our device several times on the same input data $x$ we get different results $y$ with probability $T_{x y}$. The crucial point is now that we can allow some of the outcomes to be wrong as long as there is an easy way (i.e. a class $\mathbf{P}$ algorithm) to check the validity of the results. Hence we define BPP ("bounded error probabilistic polynomial time") as the class of all decision problems which admit a polynomial time probabilistic algorithm with error probability less than $1 / 2-\epsilon$ (for fixed $\epsilon$ ). It is obvious that $\mathbf{P} \subset \mathbf{B P P}$ holds but the relation between $\mathbf{B P P}$ and $\mathbf{N P}$ is not known.

4.5.3. Reversible computing. - In the last subsection we have discussed the time needed to perform a certain computation. Other physical quantities which seem to be important are space and energy. Space can be treated in a similar way as time and there are in fact space-related complexity classes (e.g PSPACE which stands for "polynomial space"). Energy, however, is different, because it turns surprisingly out that it is possible to do any calculation without expending any energy! One source of energy consumption in a usual computer is the intrinsic irreversibility of the basic operations. E.g. a basic gate like AND maps two input bits to one output bit, which implies obviously that the input can not be reconstructed from

\footnotetext{
${ }^{2}$ Note that we have glanced over a lot of technical problems at this point. The crucial difficulty is that each circuit $C_{N}$ allows only the computation of a boolean function $f_{N}: \mathbb{B}^{N} \rightarrow \mathbb{B}$ which acts on input data of length $N$. Since we are interested in answers for arbitrary finite lengthinputs a sequence $C_{N}, N \in \mathbb{N}$ of circuits with appropriate uniformity properties is needed; cf. 124 for details.
} 
the output. In other words: one bit of information is erased during the operation of the AND gate, hence a small amount of energy is dissipated to the environment. A thermodynamic analysis, known as Landauer's principle, shows that this energy loss is at least $k_{B} T \ln 2$, where $T$ is the temperature of the environment 106.

If we want to avoid this kind of energy dissipation we are restricted to reversible processes, i.e. it should be possible to reconstruct the input data from the output data. This is called reversible computation and it is performed in terms of reversible gates, which in turn can be described by invertible functions $f: \mathbb{B}^{N} \rightarrow \mathbb{B}^{N}$. This does not restrict the class of problems which can be solved however: We can repackage a non-invertible function $f: \mathbb{B}^{N} \rightarrow \mathbb{B}^{M}$ into an invertible one $f^{\prime}: \mathbb{B}^{N+M} \rightarrow \mathbb{B}^{N+M}$ simply by $f^{\prime}(x, 0)=(x, f(x))$ and an appropriate extension to the rest of $\mathbb{B}^{N+M}$. It can be even shown that a reversible computer performs as good as a usual one, i.e. an "irreversible" network can be simulated in polynomial time by a reversible one. This will be of particular importance for quantum computing, because a reversible computer is, as we will see soon, a special case of a quantum computer.

4.5.4. The network model of a quantum computer. - Now we are ready to introduce a mathematical model for quantum computation. To this end we will generalize the network model discussed in Subsection 4.5.1 to the network model of quantum computation.

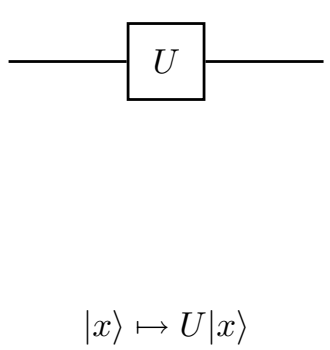

One qubit gate.

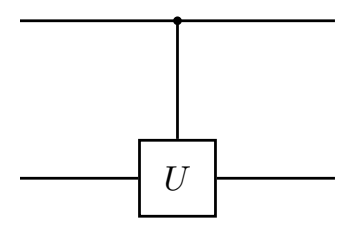

$$
|0 x\rangle \mapsto|0\rangle \otimes|x\rangle
$$$$
|1 x\rangle \mapsto|1\rangle \otimes U|x\rangle
$$

Controlled $U$ gate.

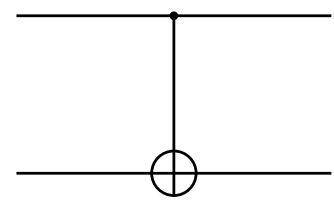

$$
\begin{aligned}
& |0 x\rangle \mapsto|0\rangle \otimes|x\rangle \\
& |1 x\rangle \mapsto|1\rangle \otimes|\neg x\rangle
\end{aligned}
$$

CNOT gate.

Figure 4.8: Universal sets of quantum gates.

A classical computer operates by a network of gates on a finite number of classical bits. A quantum computer operates on a finite number of qubits in terms of a network of quantum gates - this is the rough idea. To be more precise consider the Hilbert space $\mathcal{H}^{\otimes N}$ with $\mathcal{H}=\mathbb{C}^{2}$ which describes a quantum register consisting of $N$ qubits. In $\mathcal{H}$ there is a preferred set $|0\rangle,|1\rangle$ of orthogonal states, describing the two values a classical bit can have. Hence we can describe each possible value $x$ of a classical register of length $N$ in terms of the computational basis $|x\rangle=$ $\left|x_{1}\right\rangle \otimes \cdots \otimes\left|x_{N}\right\rangle, x \in \mathbb{B}^{N}$. A quantum gate is now nothing else but a unitary operator acting on a small number of qubits (preferably 1 or 2 ) and a quantum network is a graph representing the composition of elementary gates taken from a small set $G$ of unitaries. A quantum computation can now be defined as the application of such a network to an input state $\psi$ of the quantum register (cf. Figure 4.9 for an example). Similar to the classical case the set $G$ should be universal; i.e. each unitary operator on a quantum register of arbitrary length can be represented as a composition of elements from $G$. Since the group of unitaries on a Hilbert space is continuous, it is not possible to do this with a finite set $G$. However we can find at least suitably small sets which have the chance to be realizable technically (e.g. in an ion-trap) 
somehow in the future. Particular examples are on the one hand the controlled $U$ operations and the set consisting of CNOT and all one-qubit gates on the other (cf. Figure 4.8; for a proof of universality see Section 4.5 of [122]).

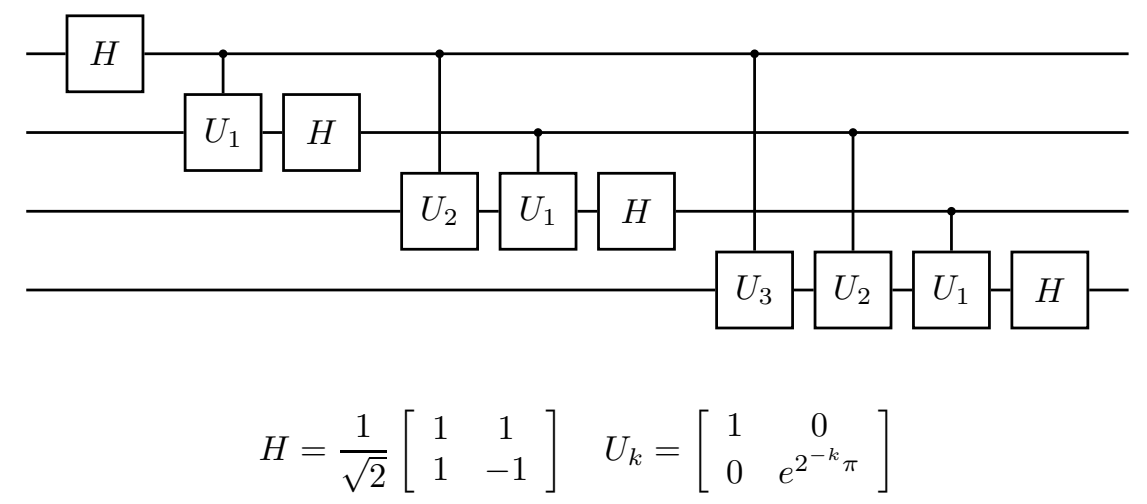

Figure 4.9: Quantum circuit for the discrete Fourier transform on a 4-qubit register.

Basically we could have considered arbitrary quantum operations instead of only unitaries as gates. We have seen however in Subsection 3.2.1 that we can implement each operation unitarily if we add an ancilla to the systems. Hence this kind of generalization is already covered by the model. (As long as non-unitarily implemented operations are a desired feature. Decoherence effect due to unavoidable interaction with the environment are a completely different story; we come back to this point at the end of the Subsection.) The same holds for measurements at intermediate steps and subsequent conditioned operations. In this case we get basically the same result with a different network where all measurements are postponed to the end. (Often it is however very useful to allow measurements at intermediate steps as we will see in the next Subsection.)

Having a mathematical model of quantum computers in mind we are now ready to discuss how it would work in principle.

1. The first step is in most cases preprocessing of the input data on a classical computer. E.g. the Shor algorithm for the factoring problem does not work if the input number $m$ is a pure prime power. However in this case there is an efficient classical algorithm. Hence we have to check first whether $m$ is of this particular form and use this classical algorithm where appropriate.

2. Based on these preprocessed data we have to prepare the quantum register in the next step. This means in the most simple case to write classical data, i.e. to prepare the state $|x\rangle \in \mathcal{H}^{\otimes N}$ if the (classical) input is $x \in \mathbb{B}^{N}$. In many cases however it might be more intelligent to use a superposition of several $|x\rangle$, e.g. the state

$$
\Psi=\frac{1}{\sqrt{2^{N}}} \sum_{x \in \mathbb{B}^{N}}|x\rangle,
$$

which represents actually the superposition of all numbers the registers can represent - this is indeed the crucial point of quantum computing and we come back to it below.

3. Now we can apply the quantum circuit $C$ to the input state $\psi$ and after the calculation we get the output state $U \psi$, where $U$ is the unitary represented by $C$. 
4. To read out the data after the calculation we perform a von Neumann measurement in the computational basis, i.e. we measure the observable given by the one dimensional projectors $|x\rangle\langle x|, x \in \mathbb{B}^{N}$. Hence we get $x \in \mathbb{B}^{N}$ with probability $P_{N}=|\langle\psi \mid x\rangle|^{2}$.

5. Finally we have to postprocess the measured value $x$ on a classical computer to end up with the final result $x^{\prime}$. If, however, the output state $U \Psi$ is a proper superposition of basis vectors $|x\rangle$ (and not just one $|x\rangle$ ) the probability $p_{x}$ to get this particular $x^{\prime}$ is less than 1 . In other words we have performed a probabilistic calculation as described in the last paragraph of Subsection 4.5.2. Hence we have to check the validity of the results (with a class $\mathbf{P}$ algorithm on a classical computer) and if they are wrong we have to go back to step 2 .

So, why is quantum computing potentially useful? First of all, a quantum computer can perform at least as good as a classical computer. This follows immediately from our discussion of reversible computing in Subsection 4.5.3 and the fact that any invertible function $f: \mathbb{B}^{N} \rightarrow \mathbb{B}^{N}$ defines a unitary by $U_{f}:|x\rangle \mapsto|f(x)\rangle$ (the quantum CNOT gate in Figure 4.8 arises exactly in this way from the classical CNOT). But, there is on the other hand strong evidence which indicates that a quantum computer can solve problems in polynomial time which a classical computer can not. The most striking example for this fact is the Shor algorithm, which provides a way to solve the factoring problem (which is most probably not in class $\mathbf{P}$ ) in polynomial time. If we introduce the new complexity class BQP of decision problems which can be solved with high probability and in polynomial time with a quantum computer, we can express this conjecture as $\mathbf{B P P} \neq \mathbf{B Q P}$.

The mechanism which gives a quantum computer its potential power is the ability to operate not just on one value $x \in \mathbb{B}^{N}$, but on whole superpositions of values, as already mentioned in step 2 above. E.g. consider a, not necessarily invertible, map $f: \mathbb{B}^{N} \rightarrow \mathbb{B}^{M}$ and the unitary operator $U_{f}$

$$
\mathcal{H}^{\otimes N} \otimes \mathcal{H}^{\otimes M} \ni|x\rangle \otimes|0\rangle \mapsto U_{f}|x\rangle \otimes|0\rangle=|x\rangle \otimes|f(x)\rangle \in \mathcal{H}^{\otimes N} \otimes \mathcal{H}^{\otimes M}
$$

If we let act $U_{f}$ on a register in the state $\Psi \otimes|0\rangle$ from Equation (4.33) we get the result

$$
U_{f}(\Psi \otimes|0\rangle)=\frac{1}{\sqrt{2^{N}}} \sum_{x \in \mathbb{B}^{N}}|x\rangle \otimes|f(x)\rangle
$$

Hence a quantum computer can evaluate the function $f$ on all possible arguments $x \in \mathbb{B}^{N}$ at the same time! To benefit from this feature - usually called quantum parallelism - is, however, not as easy as it looks like. If we perform a measurement on $U_{f}(\Psi \otimes|0\rangle)$ in the computational basis we get the value of $f$ for exactly one argument and the rest of the information originally contained in $U_{f}(\Psi \otimes|0\rangle)$ is destroyed. In other words it is not possible to read out all pairs $(x, f(x))$ from $U_{f}(\Psi \otimes|0\rangle)$ and to fill a (classical) lookup table with them. To take advantage from quantum parallelism we have to use a clever algorithm within the quantum computation step (step 3 above). In the next section we will consider a particular example for this.

Before we come to this point, let us give some additional comments which link this section to other parts of quantum information. The first point concerns entanglement. The state $U_{f}(\Psi \otimes|0\rangle)$ is highly entangled (although $\Psi$ is separable since $\left.\Psi=\left[2^{-1 / 2}(|0\rangle+|1\rangle)\right]^{\otimes N}\right)$, and this fact is essential for the "exponential speedup" of computations we could gain in a quantum computer. In other words, to outperform a classical computer, entanglement is the most crucial resource - this will become more transparent in the next section. The second remark concerns error 
correction. Up to now we have assumed implicitly all components of a quantum computer work perfectly without any error. In reality however decoherence effects make it impossible to realize unitarily implemented operations, and we have to deal with noisy channels. Fortunately it is possible within quantum information to correct at least a certain amount of errors, as we have seen in Section 4.4). Hence unlike an analog computerf a quantum computer can be designed fault tolerant, i.e. it can work with imperfectly manufactured components.

4.5.5. Simons problem. - We will consider now a particular problem (known as Simons problem; cf. [143]) which shows explicitly how a quantum computer can speed up a problem which is hard to solve with a classical computer. It does not fit however exactly into the general scheme sketched in the last subsection, because a quantum "oracle" is involved, i.e. a black box which performs an (a priori unknown) unitary transformation on an input state given to it. The term "oracle" indicates here that we are not interested in the time the black box needs to perform the calculation but only in the number of times we have to access it. Hence this example does not prove the conjecture $\mathbf{B P P} \neq \mathbf{B Q P}$ stated above. Other quantum algorithms which we have not the room here to discuss include: the Deutsch [52] and Deutsch-Josza problem [53, the Grover search algorithm [75, 74 and of course Shor's factoring algorithm [139, 140].

Hence let us assume that our black box calculates the unitary $U_{f}$ from Equation (4.34) with a map $f: \mathbb{B}^{N} \rightarrow \mathbb{B}^{N}$ which is two to one and has period $a$, i.e. $f(x)=$ $f(y)$ iff $y=x+a \bmod 2$. The task is to find $a$. Classically, this problem is hard, i.e. we have to query the oracle exponentially often. To see this note first that we have to find a pair $(x, y)$ with $f(x)=f(y)$ and the probability to get it with two random queries is $2^{-N}$ (since there is for each $x$ exactly one $y \neq x$ with $f(x)=f(y)$ ). If we use the box $2^{N / 4}$ times, we get less than $2^{N / 2}$ different pairs. Hence the probability to get the correct solution is $2^{-N / 2}$, i.e. arbitrarily small even with exponentially many queries.

Assume now that we let our box act on a quantum register $\mathcal{H}^{\otimes N} \otimes \mathcal{H}^{\otimes N}$ in the state $\Psi \otimes|0\rangle$ with $\Psi$ from Equation (4.33) to get $U_{f}(\Psi \otimes|0\rangle)$ from (4.35). Now we measure the second register. The outcome is one of $2^{N-1}$ possible values (say $f\left(x_{0}\right)$ ), each of which occurs equiprobable. Hence, after the measurement the first register is the state $2^{-1 / 2}(|x\rangle+|x+a\rangle)$ ). Now we let a Hadamard gate $H$ (cf. Figure 4.9) act on each qubit of the first register and the result is (this follows with a short calculation)

$$
\frac{1}{\sqrt{2}} H^{\otimes N}(|x\rangle+|x+a\rangle)=\frac{1}{\sqrt{2^{N-1}}} \sum_{a \cdot y=0}(-1)^{x \cdot y}|y\rangle
$$

where the dot denotes the ( $\mathbb{B}$-valued) scalar product in the vector space $\mathbb{B}^{N}$. Now we perform a measurement on the first register (in computational basis) and we get a $y \in \mathbb{B}^{N}$ with the property $y \cdot a=0$. If we repeat this procedure $N$ times and if we get $N$ linear independent values $y_{j}$ we can determine $a$ as a solution of the system of equations $y_{1} \cdot a=0, \ldots, y_{N} \cdot a=0$. The probability to appear as an outcome of the second measurement is for each $y$ with $y \cdot a=0$ given by $2^{1-N}$. Therefore the success probability can be made arbitrarily big while the number of times we have to access the box is linear in $N$.

\subsection{Quantum cryptography}

Finally we want to have a short look on quantum cryptography - another more practical application of quantum information, which has the potential to emerge into

\footnotetext{
${ }^{3}$ If an analog computer works reliably only with a certain accuracy, we can rewrite the algorithm into a digital one.
} 
technology in the not so distant future (see e.g. 95, 93, 34] for some experimental realizations and [69] for a more detailed overview). Hence let us assume that Alice has a message $x \in \mathbb{B}^{N}$ which she wants to send secretly to Bob over a public communication channels. One way to do this is the so called "one-time pad": Alice generates randomly a second bit-string $y \in \mathbb{B}^{N}$ of the same length as $x$ sends $x+y$ instead of $x$. Without knowledge of the key $y$ it is completely impossible to recover the message $x$ from $x+y$. Hence this is a perfectly secure method to transmit secret data. Unfortunately it is completely useless without a secure way to transmit the key $y$ to Bob, because Bob needs $y$ to decrypt the message $x+y$ (simply by adding $y$ again). What makes the situation even worse is the fact that the key $y$ can be used only once (therefore the name one-time pad). If two messages $x_{1}, x_{2}$ are encrypted with the same key we can use $x_{1}$ as a key to decrypt $x_{2}$ and vice versa: $\left(x_{1}+y\right)+\left(x_{2}+y\right)=x_{1}+x_{2}$, hence both messages are partly compromised.

Due to these problems completely different approaches, namely "public key systems" like DSA and RSA are used today for cryptography. The idea is to use two keys instead of one: a private key which is used for decryption and only known to its owner and a public key used for encryption, which is publicly available (we do not discuss the algorithms needed for key generation, encryption and decryption here, see [145] and the references therein instead). To use this method, Bob generates a key pair $(z, y)$, keeps his private key $(y)$ at a secure place and sends the public one $(z)$ to Alice over a public channel. Alice encrypts her message with $z$ sends the result to Bob and he can decrypt it with $y$. The security of this scheme relies on the assumption that the factoring problem is computationally hard, i.e. not in class $\mathbf{P}$, because to calculate $y$ from $z$ requires the factorization of large integers. Since the latter is tractable on quantum computers via Shor's algorithm, the security of public key systems breaks down if quantum computers become available in the future. Another problem of more fundamental nature is the unproven status of the conjecture that factorization is not solvable in polynomial time. Consequently, security of public key systems is not proven either.

The crucial point is now that quantum information provides a way to distribute a cryptographic key $y$ in a secure way, such that $y$ can be used as a one-time pad afterwards. The basic idea is to use the no cloning theorem to detect possible eavesdropping attempts. To make this more transparent, let us consider a particular example here, namely the probably most prominent protocol proposed by Benett and Brassard in 1984 [10].

1. Assume that Alice wants to transmit bits from the (randomly generated) key $y \in \mathbb{B}^{N}$ through an ideal quantum channel to Bob. Before they start they settle upon two orthonormal bases $e_{0}, e_{1} \in \mathcal{H}$, respectively $f_{0}, f_{1} \in \mathcal{H}$, which are mutually nonorthogonal, i.e. $\left|\left\langle e_{j}, f_{k}\right\rangle\right| \geq \epsilon>0$ with $\epsilon$ big enough for each $j, k=0,1$. If photons are used as information carrier a typical choice are linearly polarized photons with polarization direction rotated by $45^{\circ}$ against each other.

2. To send one bit $j \in \mathbb{B}$ Alice selects now at random one of the two bases, say $e_{0}, e_{1}$ and then she sends a qubit in the state $\left|e_{j}\right\rangle\left\langle e_{j}\right|$ through the channel. Note that neither Bob nor a potential eavesdropper knows which bases she has chosen.

3. When Bob receives the qubit he selects, as Alice before, at random a base and performs the corresponding von Neumann measurement to get one classical bit $k \in \mathbb{B}$, which he records together with the measurement method.

4. Both repeat this procedure until the whole string $y \in \mathbb{B}^{N}$ is transmitted and then Bob tells Alice (through a classical, public communication channel) 
bit for bit which base he has used for the measurement (but not the result of the measurement). If he has used the same base as Alice both keep the corresponding bit otherwise they discard it. They end up with a bit-string $y^{\prime} \in \mathbb{B}^{M}$ of a reduced length $M$. If this is not sufficient they have to continue sending random bits until the key is long enough. For large $N$ the rate of successfully transmitted bits per bits sended is obviously $1 / 2$. Hence Alice has to send approximately twice as many bits as they need.

To see why this procedure is secure, assume now that the eavesdropper Eve can listen and modify the information sent through the quantum channel and that she can listen on the classical channel but can not modify it (we come back to this restriction in a minute). Hence Eve can intercept the qubits sent by Alice and make two copies of it. One she forwards to Bob and the other she keeps for later analysis. Due to the no cloning theorem however she has produced errors in both copies and the quality of her own decreases if she tries to make the error in Bob's as small as possible. Even if Eve knows about the two bases $e_{0}, e_{1}$ and $f_{0}, f_{1}$ she does not know which one Alice uses to send a particular qubit . Hence Eve has to decide randomly which base to choose (as Bob). If $e_{0}, e_{1}$ and $f_{0}, f_{1}$ are chosen optimal, i.e. $\left|\left\langle e_{j}, f_{k}\right\rangle\right|^{2}=0.5$ it is easy to see that the error rate Eve necessarily produces if she randomly measures in one of the bases is $1 / 4$ for large $N$. To detect this error Alice and Bob simply have to sacrify portions of the generated key and to compare randomly selected bits using their classical channel. If the error rate they detect is too big they can decide to drop the whole key and restart from the beginning.

So let us discuss finally a situation where Eve is able to intercept the quantum and the classical channel. This would imply that she can play Bob's part for Alice and Alice's for Bob. As a result she shares a key with Alice and one with Bob. Hence she can decode all secret data Alice sends to Bob, read it, and encode it finally again to forward it to Bob. To secure against such a "woman in the middle attack", Alice and Bob can use classical authentication protocols which ensure that the correct person is at the other end of the line. This implies that they need a small amount of initial secret material which can be renewed however from the new key they have generated through quantum communication.

\footnotetext{
${ }^{4}$ If Alice and Bob uses only one basis to send the data and Eve knows about it she can produce of course ideal copies of the qubits. This is actually the reason why two nonorthogonal bases are necessary.
} 


\section{Chapter 5}

\section{Entanglement measures}

We have seen in the last chapter that entanglement is an essential resource for many tasks of quantum information theory, like teleportation or quantum computation. This means that entangled states are needed for the functioning of many processes and that they are consumed during operation. It is therefore necessary to have measures which tell us whether the entanglement contained in a number of quantum systems is sufficient to perform a certain task. What makes this subject difficult, is the fact that we can not restrict the discussion to systems in a maximally or at least highly entangled pure state. Due to unavoidable decoherence effects realistic applications have to deal with imperfect systems in mixed states, and exactly in this situation the question for the amount of available entanglement is interesting.

\subsection{General properties and definitions}

The difficulties arising if we try to quantify entanglement can be divided, roughly speaking, into two parts: First we have to find a reasonable quantity which describes exactly those properties which we are interested in and second we have to calculate it for a given state. In this section we will discuss the first problem and consider several different possibilities to define entanglement measures.

5.1.1. Axiomatics. - First of all, we will collect some general properties which a reasonable entanglement measure should have (cf. also [16, 154, 153, 155, 89]). To quantify entanglement, means nothing else but to associate a positive real number to each state of (finite dimensional) two-partite systems.

Axiom E0 An entanglement measure is a function $E$ which assigns to each state $\rho$ of a finite dimensional bipartite system a positive real number $E(\rho) \in \mathbb{R}^{+}$.

Note that we have glanced over some mathematical subtleties here, because $E$ is not just defined on the state space of $\mathcal{B}(\mathcal{H} \otimes \mathcal{K})$ systems for particularly chosen Hilbert spaces $\mathcal{H}$ and $\mathcal{K}-E$ is defined on any state space for arbitrary finite dimensional $\mathcal{H}$ and $\mathcal{K}$. This is expressed mathematically most conveniently by a family of functions which behaves naturally under restrictions (i.e. the restriction to a subspace $\mathcal{H}^{\prime} \otimes \mathcal{K}^{\prime}$ coincides with the function belonging to $\left.\mathcal{H}^{\prime} \otimes \mathcal{K}^{\prime}\right)$. However we will see soon that we can safely ignore this problem.

The next point concerns the range of $E$. If $\rho$ is unentangled $E(\rho)$ should be zero of course and it should be maximal on maximally entangled states. But what happens if we allow the dimensions of $\mathcal{H}$ and $\mathcal{K}$ to grow? To get an answer consider first a pair of qubits in a maximally entangled state $\rho$. It should contain exactly one bit entanglement i.e. $E(\rho)=1$ and $N$ pairs in the state $\rho^{\otimes N}$ should contain $N$ bits. If we interpret $\rho^{\otimes N}$ as a maximally entangled state of a $\mathcal{H} \otimes \mathcal{H}$ system with $\mathcal{H}=\mathbb{C}^{N}$ we get $E\left(\rho^{\otimes N}\right)=\log _{2}(\operatorname{dim}(\mathcal{H}))=N$, where we have to reshuffle in $\rho^{\otimes N}$ the tensor factors such that $\left(\mathbb{C}^{2} \otimes \mathbb{C}^{2}\right)^{\otimes N}$ becomes $\left(\mathbb{C}^{2}\right)^{\otimes N} \otimes\left(\mathbb{C}^{2}\right)^{\otimes N}$ (i.e. "all Alice particles to the left and all Bob particles to the right"; cf. Section 4.3.) This observation motivates the following.

Axiom E1 (Normalization) E vanishes on separable and takes its maximum on maximally entangled states. This means more precisely that $E(\sigma) \leq E(\rho)=\log _{2}(d)$ for $\rho, \sigma \in \mathcal{S}(\mathcal{H} \otimes \mathcal{H})$ and $\rho$ maximally entangled.

One thing an entanglement measure should tell us, is how much quantum information can be maximally teleported with a certain amount of entanglement, where 
this maximum is taken over all possible teleportation schemes and distillation protocols, hence it can not be increased further by additional LOCC operations on the entangled systems in question. This consideration motivates the following Axiom.

Axiom E2 (LOCC MONOTONICITY) E can not increase under LOCC operation, i.e. $E[T(\rho)] \leq E(\rho)$ for all states $\rho$ and all LOCC channels $T$.

A special case of LOCC operations are of course local unitary operations $U \otimes V$. Axiom E2 implies now that $E\left(U \otimes V \rho U^{*} \otimes V^{*}\right) \leq E(\rho)$ and on the other hand $E\left(U^{*} \otimes V^{*} \widetilde{\rho} U \otimes V\right) \leq E(\widetilde{\rho})$ hence with $\widetilde{\rho}=U \otimes V \rho U^{*} \otimes V$ we get $E(\rho) \leq E(U \otimes$ $\left.V \rho V^{*} \otimes U^{*}\right)$ therefore $E(\rho)=E\left(U \otimes V \rho U^{*} \otimes V^{*}\right)$. We fix this property as a weakened version of Axiom E2:

AXIOM E2A (LOCAL UNITARY INVARIANCE) $E$ is invariant under local unitaries, i.e. $E\left(U \otimes V \rho U^{*} \otimes V^{*}\right)=E(\rho)$ for all states $\rho$ and all unitaries $U, V$.

This axiom shows why we do not have to bother about families of functions as mentioned above. If $E$ is defined on $\mathcal{S}(\mathcal{H} \otimes \mathcal{H})$ it is automatically defined on $\mathcal{S}\left(\mathcal{H}_{1} \otimes \mathcal{H}_{2}\right)$ for all Hilbert spaces $\mathcal{H}_{k}$ with $\operatorname{dim}\left(\mathcal{H}_{k}\right) \leq \operatorname{dim}(\mathcal{H})$, because we can embed $\mathcal{H}_{1} \otimes \mathcal{H}_{2}$ under this condition unitarily into $\mathcal{H} \otimes \mathcal{H}$.

Consider now a convex linear combination $\lambda \rho+(1-\lambda) \sigma$ with $0 \leq \lambda \leq 1$. Entanglement can not be "generated" by mixing two states, i.e. $E(\lambda \rho+(1-\lambda) \sigma) \leq$ $\lambda E(\rho)+(1-\lambda) E(\sigma)$.

Axiom E3 (Convexity) $E$ is a convex function, i.e. $E(\lambda \rho+(1-\lambda) \sigma) \leq \lambda E(\rho)+$ $(1-\lambda) E(\sigma)$ for two states $\rho, \sigma$ and $0 \leq \lambda \leq 1$.

The next property concerns the continuity of $E$, i.e. if we perturb $\rho$ slightly the change of $E(\rho)$ should be small. This can be expressed most conveniently as continuity of $E$ in the trace norm. At this point however it is not quite clear, how we have to handle the fact that $E$ is defined for arbitrary Hilbert spaces. The following version is motivated basically by the fact that it is a crucial assumption in Theorem 5.1 .2 and 5.1.3.

Axiom E4 (Continuity) Consider a sequence of Hilbert spaces $\mathcal{H}_{N}, N \in \mathbb{N}$ and two sequences of states $\rho_{N}, \sigma_{N} \in \mathcal{S}\left(\mathcal{H}_{N} \otimes \mathcal{H}_{N}\right)$ with $\lim \left\|\rho_{N}-\sigma_{N}\right\|_{1}=0$. Then we have

$$
\lim _{N \rightarrow \infty} \frac{E\left(\rho_{N}\right)-E\left(\sigma_{N}\right)}{1+\log _{2}\left(\operatorname{dim} \mathcal{H}_{N}\right)}=0 .
$$

The last point we have to consider here are additivity properties: Since we are looking at entanglement as a resource, it is natural to assume that we can do with two pairs in the state $\rho$ twice as much as with one $\rho$, or more precisely $E(\rho \otimes \rho)=$ $2 E(\rho)$ (in $\rho \otimes \rho$ we have to reshuffle tensor factors again ;see above).

Axiom E5 (AdDitivity) For any pair of two-partite states $\rho, \sigma \in \mathcal{S}(\mathcal{H} \otimes \mathcal{K})$ we have $E(\sigma \otimes \rho)=E(\sigma)+E(\rho)$.

Unfortunately this rather natural looking axiom seems to be too strong (it excludes reasonable candidates). It should be however always true that entanglement can not increase if we put two pairs together.

Axiom E5A (Subadditivity) For any pair of states $\rho, \sigma$ we have $E(\rho \otimes \sigma) \leq$ $E(\rho)+E(\sigma)$.

There are further modifications of additivity available in the literature. Most frequently used is the following, which restricts Axiom E5 to the case $\rho=\sigma$ :

Axiom E5B (WEAK ADDITIVITy) For any state $\rho$ of a bipartite system we have $N^{-1} E\left(\rho^{\otimes N}\right)=E(\rho)$. 
Finally, the weakest version of additivity only deals with the behavior of $E$ for large tensor products, i.e. $\rho^{\otimes N}$ for $N \rightarrow \infty$.

Axiom E5C (Existence of A Regularization) For each state $\rho$ the limit

$$
E^{\infty}(\rho)=\lim _{N \rightarrow \infty} \frac{E\left(\rho^{\otimes N}\right)}{N}
$$

exists.

5.1.2. Pure states. - Let us consider now a pure state $\rho=|\psi\rangle\langle\psi| \in \mathcal{S}(\mathcal{H} \otimes$ $\mathcal{K})$. If it is entangled its partial trace $\sigma=\operatorname{tr}_{\mathcal{H}}|\psi\rangle\left\langle\psi\left|=\operatorname{tr}_{\mathcal{K}}\right| \psi\right\rangle\langle\psi|$ is mixed and for a maximally entangled state it is maximally mixed. This suggests to use the von Neumann entropy ${ }^{円}$ of $\rho$, which measures how much a state is mixed, as an entanglement measure for mixed states, i.e. we define 9,16

$$
E_{\mathrm{vN}}(\rho)=-\operatorname{tr}\left[\operatorname{tr}_{\mathcal{H}} \rho \ln \left(\operatorname{tr}_{\mathcal{H}} \rho\right)\right] .
$$

It is easy to deduce from the properties of the von Neumann entropy that $E_{\mathrm{vN}}$ satisfies Axioms E0, E1, E3 and E5b. Somewhat more difficult is only Axiom E2 which follows however from a nice theorem of Nielsen [119] which relates LOCC operations (on pure states) to the theory of majorization. To state it here we need first some terminology. Consider two probability distributions $\lambda=\left(\lambda_{1}, \ldots, \lambda_{M}\right)$ and $\mu=\left(\mu_{1}, \ldots, \mu_{N}\right)$ both given in decreasing order (i.e. $\lambda_{1} \geq \ldots \geq \lambda_{M}$ and $\left.\mu_{1} \geq \ldots \geq \mu_{N}\right)$. We say that $\lambda$ is majorized by $\mu$, in symbols $\lambda \prec \mu$, if

$$
\sum_{j=1}^{k} \lambda_{j} \leq \sum_{j=1}^{k} \mu_{j} \quad \forall k=1, \ldots, \min M, N
$$

holds. Now we have the following result (see [119] for a proof).

TheOREM 5.1.1 A pure state $\psi=\sum_{j} \lambda_{j}^{1 / 2} e_{j} \otimes e_{j}^{\prime} \in \mathcal{H} \otimes \mathcal{K}$ can be transformed into another pure state $\phi=\sum_{j} \mu_{j}^{1 / 2} f_{j} \otimes f_{j}^{\prime} \in \mathcal{H} \otimes \mathcal{K}$ via a LOCC operation, iff the Schmidt coefficients of $\psi$ are majorized by those of $\phi$, i.e. $\lambda \prec \mu$.

The von Neumann entropy of the restriction $\operatorname{tr}_{\mathcal{H}}|\psi\rangle\langle\psi|$ can be immediately calculated from the Schmidt coefficients $\lambda$ of $\psi$ by $E_{\mathrm{vN}}(|\psi\rangle\langle\psi|)=-\sum_{j} \lambda_{j} \ln \left(\lambda_{j}\right)$. Axiom E2 follows therefore from the fact that the entropy $S(\lambda)=-\sum_{j} \lambda_{j} \ln \left(\lambda_{j}\right)$ of a probability distribution $\lambda$ is a Shur concave function, i.e. $\lambda \prec \mu$ implies $S(\lambda) \geq$ $S(\mu)$; see 121.

Hence we have seen so far that $E_{\mathrm{vN}}$ is one possible candidate for an entanglement measure on pure states. In the following we will see that it is in fact the only candidate which is physically reasonable. There are basically two reasons for this. The first one deals with distillation of entanglement. It was shown by Bennett et. al. [9] that each state $\psi \in \mathcal{H} \otimes \mathcal{K}$ of a bipartite system can be prepared out of (a possibly large number of) systems in an arbitrary entangled state $\phi$ by LOCC operations. To be more precise, we can find a sequence of LOCC operations

$$
T_{N}: \mathcal{B}\left[(\mathcal{H} \otimes \mathcal{K})^{\otimes M(N)}\right] \rightarrow \mathcal{B}\left[(\mathcal{H} \otimes \mathcal{K})^{\otimes N}\right]
$$

such that

$$
\lim _{N \rightarrow \infty} \| T_{N}^{*}\left(|\phi\rangle\left\langle\left.\phi\right|^{\otimes N}\right)-|\psi\rangle\langle\psi| \|_{1}=0\right.
$$

\footnotetext{
${ }^{1}$ We assume here and in the following that the reader is sufficiently familiar with entropies. If this is not the case we refer to 123 .
} 
holds with a nonvanishing rate $r=\lim _{N \rightarrow \infty} M(N) / N$. This is done either by distillation $(r<1$ if $\psi$ is higher entangled then $\phi)$ or by "diluting" entanglement, i.e. creating many less entangled states from few highly entangled ones $(r>1)$. All this can be performed in a reversible way: We can start with some maximally entangled qubits dilute them to get many less entangled states which can be distilled afterwards to get the original states back (again only in an asymptotic sense). The crucial point is that the asymptotic rate $r$ of these processes is given in terms of $E_{\mathrm{vN}}$ by $r=E_{\mathrm{vN}}(|\phi\rangle\langle\phi|) / E_{\mathrm{vN}}(|\psi\rangle\langle\psi|)$. Hence we can say, roughly speaking that $E_{\mathrm{vN}}(|\psi\rangle\langle\psi|)$ describes exactly the amount of maximally entangled qubits which is contained in $|\psi\rangle\langle\psi|$.

A second somewhat more formal reason is that $E_{\mathrm{vN}}$ is the only entanglement measure on the set of pure states which satisfies the axioms formulated above. In other words the following "uniqueness theorem for entanglement measures" holds $129,155,57$

THEOREM 5.1.2 The reduced von Neumann entropy $E_{\mathrm{vN}}$ is the only entanglement measure on pure states which satisfies Axioms E0-ES.

5.1.3. Entanglement measures for mixed states. - To find reasonable entanglement measures for mixed states is much more difficult. There are in fact many possibilities (e.g. the maximally entangled fraction introduced in Subsection 3.1.1 can be regarded as a simple measure) and we want to present therefore only four of the most reasonable candidates. Among those measures which we do not discuss here are negativity quantities (158] and the references therein) the "best separable approximation" 108, the base norm associated with the set of separable states 157, 136 and ppt-distillation rates 133.

The first measure we want to present is oriented along the discussion of pure states: We define, roughly speaking, the asymptotic rate with which maximally entangled qubits can be distilled at most out of a state $\rho \in \mathcal{S}(\mathcal{H} \otimes \mathcal{K})$ as the Entanglement of Distillation $E_{\mathrm{D}}(\rho)$ of $\rho$; cf [12]. To be more precise consider all possible distillation protocols for $\rho$ (cf. Section 4.3), i.e. all sequences of LOCC channels

$$
T_{N}: \mathcal{B}\left(\mathbb{C}^{d_{N}} \otimes \mathbb{C}^{d_{N}}\right) \rightarrow \mathcal{B}\left(\mathcal{H}^{\otimes N} \otimes \mathcal{K}^{\otimes N}\right)
$$

such that

$$
\lim _{N \rightarrow \infty} \| T_{N}^{*}\left(\rho^{\otimes N}\right)-\left|\Omega_{N}\right\rangle\left\langle\Omega_{N}\right| \|_{1}=0
$$

holds with a sequence of maximally entangled states $\Omega_{N} \in \mathbb{C}^{d_{N}}$. Now we can define

$$
E_{\mathrm{D}}(\rho)=\sup _{\left(T_{N}\right)_{N \in \mathbb{N}}} \limsup _{N \rightarrow \infty} \frac{\log _{2}\left(d_{N}\right)}{N}
$$

where the supremum is taken over all possible distillation protocols $\left(T_{N}\right)_{N \in \mathbb{N}}$. It is not very difficult to see that $E_{\mathrm{D}}$ satisfies E0, E1, E2 and E5b. It is not known whether continuity (E4) and convexity (Axiom E3) holds. It can be shown however that $E_{\mathrm{D}}$ is not convex (and not additive; Axiom E5) if npt bound entangled states exist (see [141], cf. also Subsection 4.3.3).

For pure states we have discussed beside distillation the "dilution" of entanglement and we can use, similar to $E_{\mathrm{D}}$, the asymptotic rate with which bipartite systems in a given state $\rho$ can be prepared out of maximally entangled singlets 78 . Hence consider again a sequence of LOCC channels

$$
T_{N}: \mathcal{B}\left(\mathcal{H}^{\otimes N} \otimes \mathcal{K}^{\otimes N}\right) \rightarrow \mathcal{B}\left(\mathbb{C}^{d_{N}} \otimes \mathbb{C}^{d_{N}}\right)
$$


and a sequence of maximally entangled states $\Omega_{N} \in \mathbb{C}^{d_{N}}, N \in \mathbb{N}$, but now with the property

$$
\lim _{N \rightarrow \infty}\left\|\rho^{\otimes N}-T_{N}^{*}\left(\left|\Omega_{N}\right\rangle\left\langle\Omega_{N}\right|\right)\right\|_{1}=0
$$

Then we can define the entanglement cost $E_{\mathrm{C}}(\rho)$ of $\rho$ as

$$
E_{\mathrm{C}}(\rho)=\inf _{\left(S_{N}\right)_{N \in \mathbb{N}}} \liminf _{N \rightarrow \infty} \frac{\log _{2}\left(d_{N}\right)}{N}
$$

where the infimum is taken over all dilution protocols $S_{N}, N \in \mathbb{N}$. It is again easy to see that $E_{\mathrm{C}}$ satisfies $\mathrm{E} 0, \mathrm{E} 1, \mathrm{E} 2$ and $\mathrm{E} 5 \mathrm{~b}$. In contrast to $E_{\mathrm{D}}$ however it can be shown that $E_{\mathrm{C}}$ is convex (Axiom E3), while it is not known, whether $E_{\mathrm{C}}$ is continuous (Axiom E4); cf [78] for proofs.

$E_{\mathrm{D}}$ and $E_{\mathrm{C}}$ are based directly on operational concepts. The remaining two measures we want to discuss here are defined in a more abstract way. The first can be characterized as the minimal convex extension of $E_{\mathrm{vN}}$ to mixed states: We define the entanglement of formation $E_{\mathrm{F}}$ of $\rho$ as $[16$

$$
E_{\mathrm{F}}(\rho)=\inf _{\rho=\sum_{j} p_{j}\left|\psi_{j}\right\rangle\left\langle\psi_{j}\right|} \sum p_{j} E_{\mathrm{vN}}\left(\left|\psi_{j}\right\rangle\left\langle\psi_{j}\right|\right),
$$

where the infimum is taken over all decompositions of $\rho$ into a convex sum of pure states. $E_{\mathrm{F}}$ satisfies $\mathrm{E} 0$ - E4 and E5a (cf. [16 for E2 and 120 for E4 the rest follows directly from the definition). Whether $E_{\mathrm{F}}$ is (weakly) additive (Axiom $\mathrm{E} 5 \mathrm{~b}$ ) is not known. Furthermore it is conjectured that $E_{\mathrm{F}}$ coincides with $E_{\mathrm{C}}$. However proven is only the identity $E_{\mathrm{F}}^{\infty}=E_{\mathrm{C}}$, where the existence of the regularization $E_{\mathrm{F}}^{\infty}$ of $E_{\mathrm{F}}$ follows directly from subadditivity.

Another idea to quantify entanglement is to measure the "distance" of the (entangled) $\rho$ from the set of separable states $\mathcal{D}$. It hat turned out 154 that among all possible distance functions the relative entropy is physically most reasonable. Hence we define the relative entropy of entanglement as

$$
E_{\mathrm{R}}(\rho)=\inf _{\sigma \in \mathcal{D}} S(\rho \mid \sigma), \quad S(\rho \mid \sigma)=\left[\operatorname{tr}\left(\rho \log _{2} \rho-\rho \log _{2} \sigma\right)\right]
$$

where the infimum is taken over all separable states. It can be shown that $E_{\mathrm{R}}$ satisfies, as $E_{\mathrm{F}}$ the Axioms E0 - E4 and E5a, where E1 and E2 are shown in 154 and E4 in [56]; the rest follows directly from the definition. It is shown in 159 that $E_{\mathrm{R}}$ does not satisfy E5b; cf. also Subsection 5.3. Hence the regularization $E_{\mathrm{R}}^{\infty}$ of $E_{\mathrm{R}}$ differs from $E_{\mathrm{R}}$.

Finally let us give now some comments on the relation between the measures just introduced. On pure states all measures just discussed, coincide with the reduced von Neumann entropy - this follows from Theorem 5.1.2 and the properties stated in the last Subsection. For mixed states the situation is more difficult. It can be shown however that $E_{\mathrm{D}} \leq E_{\mathrm{C}}$ holds and that all "reasonable" entanglement measures lie in between 89 .

THEOREM 5.1.3 For each entanglement measure E satisfying Ed, EA, EG and E5b and each state $\rho \in \mathcal{S}(\mathcal{H} \otimes \mathcal{K})$ we have $E_{\mathrm{D}}(\rho) \leq E(\rho) \leq E_{\mathrm{C}}(\rho)$.

Unfortunately no measure we have discussed in the last Subsection satisfies all the assumptions of the theorem. It is possible however to get a similar statement for the regularization $E^{\infty}$ with weaker assumptions on $E$ itself (in particular without assuming additivity); cf [57. 


\subsection{Two qubits}

Even more difficult than finding reasonable entanglement measures are explicit calculations. All measures we have discussed above involve optimization processes over spaces which grow exponentially with the dimension of the Hilbert space. A direct numerical calculation for a general state $\rho$ is therefore hopeless. There are however some attempts to get either some bounds on entanglement measures or to get explicit calculations for special classes of states. We will concentrate this discussion to some relevant special cases. On the one hand we will concentrate on $E_{\mathrm{F}}$ and $E_{\mathrm{R}}$ and on the other we will look at two special classes of states where explicit calculations are possible: Two qubit systems in this section and states with symmetry properties in the next one.

5.2.1. Pure states. - Assume for the rest of this section that $\mathcal{H}=\mathbb{C}^{2}$ holds and consider first a pure state $\psi \in \mathcal{H} \otimes \mathcal{H}$. To calculate $E_{\mathrm{vN}}(\psi)$ is of course not difficult and it is straightforward to see that (cf. for all material of this and the following subsection [16]):

$$
E_{\mathrm{vN}}(\psi)=H\left[\frac{1}{2}\left(1+\sqrt{1-C(\psi)^{2}}\right)\right]
$$

holds, with

$$
H(x)=-x \log _{2}(x)-(1-x) \log _{2}(1-x)
$$

and the concurrence $C(\psi)$ of $\psi$ which is defined by

$$
C(\psi)=\left|\sum_{j=0}^{3} \alpha_{j}^{2}\right| \text { with } \psi=\sum_{j=0}^{3} \alpha_{j} \Phi_{j}
$$

where $\Phi_{j}, j=0, \ldots, 3$ denotes the Bell basis (3.3). Since $C$ becomes rather important in the following let us reexpress it as $C(\psi)=|\langle\psi, \Xi \psi\rangle|$, where $\psi \mapsto \Xi \psi$ denotes complex conjugation in Bell basis. Hence $\Xi$ is an antiunitary operator and it can be written as the tensor product $\Xi=\xi \otimes \xi$ of the map $\mathcal{H} \ni \phi \mapsto \sigma_{2} \bar{\phi}$, where $\bar{\phi}$ denotes complex conjugation in the canonical basis and $\sigma_{2}$ is the second Pauli matrix. Hence local unitaries (i.e. those of the form $U_{1} \otimes U_{2}$ ) commute with $\Xi$ and it can be shown that this is not only a necessary but also a sufficient condition for a unitary to be local 160 .

We see from Equations (5.15) and (5.17) that $C(\psi)$ ranges from 0 to 1 and that $E_{\mathrm{vN}}(\psi)$ is a monotone function in $C(\psi)$. The latter can be considered therefore as an entanglement quantity in its own right. For a Bell state we get in particular $C\left(\Phi_{j}\right)=1$ while a separable state $\phi_{1} \otimes \phi_{2}$ leads to $C\left(\phi_{1} \otimes \phi_{2}\right)=0$; this can be seen easily with the factorization $\Xi=\xi \otimes \xi$.

Assume now that one of the $\alpha_{j}$ say $\alpha_{0}$ satisfies $\left|\alpha_{0}\right|^{2}>1 / 2$. This implies that $C(\psi)$ can not be zero since

$$
\left|\sum_{j=1}^{3} \alpha_{j}^{2}\right| \leq 1-\left|\alpha_{0}\right|^{2}
$$

must hold. Hence $C(\psi)$ is at least $1-2\left|\alpha_{0}\right|^{2}$ and this implies for $E_{\mathrm{vN}}$ and arbitrary $\psi$

$$
E_{\mathrm{vN}}(\psi) \geq h\left(\left|\left\langle\Phi_{0}, \psi\right\rangle\right|^{2}\right) \text { with } h(x)=\left\{\begin{array}{ll}
H\left[\frac{1}{2}+\sqrt{x(1-x)}\right] & x \geq \frac{1}{2} \\
0 & x<\frac{1}{2}
\end{array} .\right.
$$


This inequality remains valid if we replace $\Phi_{0}$ by any other maximally entangled state $\Phi \in \mathcal{H} \otimes \mathcal{H}$. To see this note that two maximally entangled states $\Phi, \Phi^{\prime} \in \mathcal{H} \otimes \mathcal{H}$ are related (up to a phase) by a local unitary transformation $U_{1} \otimes U_{2}$ (this follows immediately from their Schmidt decomposition; cf Subsection 3.1.1). Hence, if we replace the Bell basis in Equation (5.17) by $\Phi_{j}^{\prime}=U_{1} \otimes U_{2} \Phi_{j}, j=0, \ldots, 3$ we get for the corresponding $C^{\prime}$ the equation $C^{\prime}(\psi)=\left\langle U_{1}^{*} \otimes U_{2}^{*} \psi, \Xi U_{1}^{*} \otimes U_{2}^{*} \psi\right\rangle=C(\psi)$ since $\Xi$ commutes with local unitaries. We can even replace $\left|\left\langle\Phi_{0}, \psi\right\rangle\right|^{2}$ with the supremum over all maximally entangled states and get therfore

$$
E_{\mathrm{vN}}(\psi) \geq h[\mathcal{F}(|\psi\rangle\langle\psi|)]
$$

where $\mathcal{F}(|\psi\rangle\langle\psi|)$ is the maximally entangled fraction of $|\psi\rangle\langle\psi|$ which we have introduced in Subsection 3.1.1.

To see that even equality holds in Equation (5.20) note first that it is sufficient to consider the case $\psi=a|00\rangle+b|11\rangle$ with $a, b \geq 0, a^{2}+b^{2}=1$, since each pure state $\psi$ can be brought into this form (this follows again from the Schmidt decomposition) by a local unitary transformation which on the other hand does not change $E_{\mathrm{vN}}$. The maximally entangled state which maximizes $|\langle\psi, \Phi\rangle|^{2}$ is in this case $\Phi_{0}$ and we get $\mathcal{F}(|\psi\rangle\langle\psi|)=(a+b)^{2} / 2=1 / 2+a b$. Straightforward calculations show now that $h[\mathcal{F}(|\psi\rangle\langle\psi|)]=h(1 / 2+a b)=E_{\mathrm{vN}}(\psi)$ holds as stated.

5.2.2. EOF for Bell diagonal states. - It is easy to extend the inequality (5.20) to mixed states if we use the convexity of $E_{\mathrm{F}}$ and the fact that $E_{\mathrm{F}}$ coincides with $E_{\mathrm{vN}}$ on pure states. Hence (5.20) becomes

$$
E_{\mathrm{F}}(\rho) \geq h[\mathcal{F}(\rho)] .
$$

For general two qubit states this bound is not achieved however. This can be see with the example $\rho=1 / 2\left(\left|\phi_{1}\right\rangle\left\langle\phi_{1}|+| 00\right\rangle\langle 00|\right)$, which we have considered already in the last paragraph of Subsection 3.1.1. It is easy to see that $\mathcal{F}(\rho)=1 / 2$ holds hence $h[\mathcal{F}(\rho)]=0$ but $\rho$ is entangled. Nevertheless we can show that equality holds in Equation (5.21) if we restrict it to Bell diagonal states $\rho=\sum_{j=0}^{3} \lambda j\left|\Phi_{j}\right\rangle\left\langle\Phi_{j}\right|$. To prove this statement we have to find a convex decomposition $\rho=\sum_{j} \mu_{j}\left|\Psi_{j}\right\rangle\left\langle\Psi_{j}\right|$ of such a $\rho$ into pure states $\left|\Psi_{j}\right\rangle\left\langle\Psi_{j}\right|$ such that $h[\mathcal{F}(\rho)]=\sum_{j} \mu_{j} E_{\mathrm{vN}}\left(\left|\Psi_{j}\right\rangle\left\langle\Psi_{j}\right|\right.$ holds. Since $E_{\mathrm{F}}(\rho)$ can not be smaller than $h[\mathcal{F}(\rho)]$ due to inequality (5.21) this decomposition must be optimal and equality is proven.

To find such $\Psi_{j}$ assume first that the biggest eigenvalue of $\rho$ is greater than $1 / 2$, and let, without loss of generality, be $\lambda_{1}$ this eigenvalue. A good choice for the $\Psi_{j}$ are then the eight pure states

$$
\sqrt{\lambda_{0}} \Phi_{0}+i\left(\sum_{j=1}^{3}\left( \pm \sqrt{\lambda_{j}}\right) \Phi_{j}\right)
$$

The reduced von Neumann entropy of all these states equals $h\left(\lambda_{1}\right)$, hence $\sum_{j} \mu_{j} E_{\mathrm{vN}}\left(\left|\Psi_{j}\right\rangle\left\langle\Psi_{j}\right|\right)=h\left(\lambda_{1}\right)$ and therefore $E_{\mathrm{F}}(\rho)=h\left(\lambda_{1}\right)$. Since the maximally entangled fraction of $\rho$ is obviously $\lambda_{1}$ we see that (5.21) holds with equality.

Assume now that the highest eigenvalue is less than $1 / 2$. Then we can find phase factors $\exp \left(i \phi_{j}\right)$ such that $\sum_{j=0}^{3} \exp \left(i \phi_{j}\right) \lambda_{j}=0$ holds and $\rho$ can be expressed as a convex linear combination of the states

$$
e^{i \phi_{0} / 2} \sqrt{\lambda_{0}} \Phi_{0}+i\left(\sum_{j=1}^{3}\left( \pm e^{i \phi_{j} / 2} \sqrt{\lambda_{j}}\right) \Phi_{j}\right) \text {. }
$$

The concurrence $C$ of all these states is 0 hence their entanglement is 0 by Equation (5.15), which in turn implies $E_{\mathrm{F}}(\rho)=0$. Again we see that equality is achieved in 
(5.21) since the maximally entangled fraction of $\rho$ is less than $1 / 2$. Summarizing this discussion we have shown (cf. Figure 5.1)

Proposition 5.2.1 A Bell diagonal state $\rho$ is entangled iff its highest eigenvalue $\lambda$ is greater than $1 / 2$. In this case the Entanglement of Formation of $\rho$ is given by

$$
E_{\mathrm{F}}(\rho)=H\left[\frac{1}{2}+\sqrt{\lambda(1-\lambda)}\right] .
$$

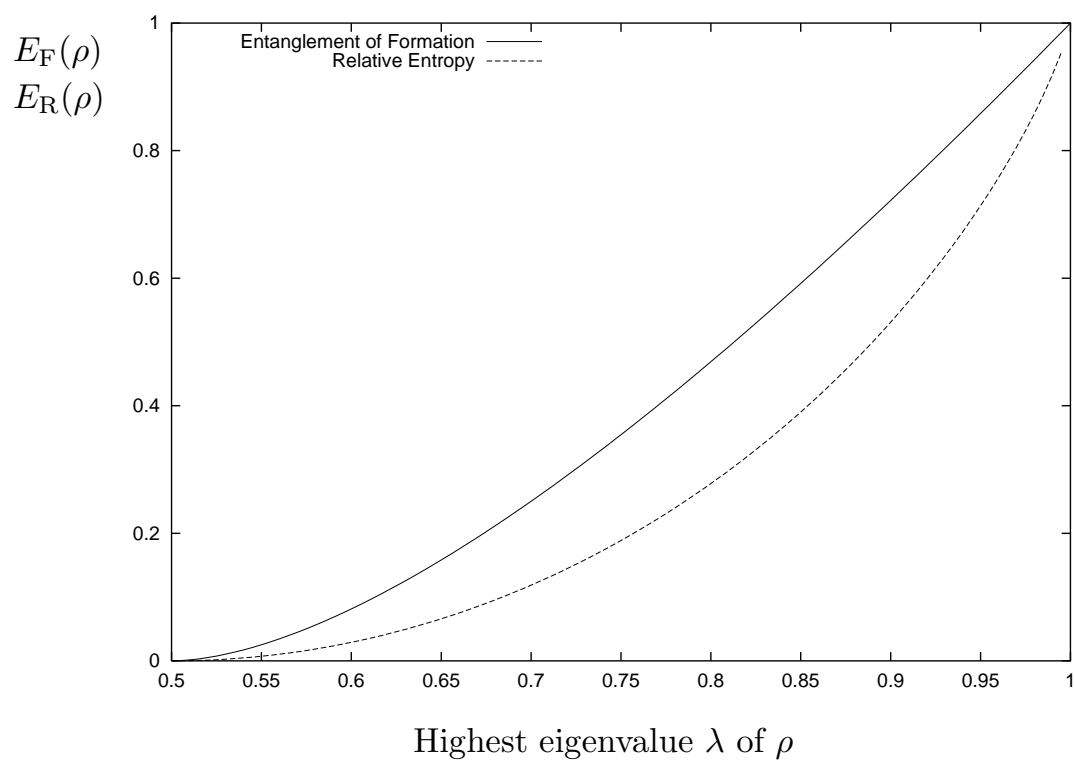

Figure 5.1: Entanglement of Formation and Relative Entropy of Entanglement for Bell diagonal states, plotted as a function of the highest eigenvalue $\lambda$ of $\rho$

5.2.3. Wootters formula. - If we have a general two qubit state $\rho$ there is a formula of Wootters 172 which allows an easy calculation of $E_{\mathrm{F}}$. It is based on a generalization of the concurrence $C$ to mixed states. To motivate it rewrite $C^{2}(\psi)=|\langle\psi, \Xi \psi\rangle|$ as

$$
C^{2}(\psi)=\operatorname{tr}(|\psi\rangle\langle\psi|| \Xi \psi\rangle\langle\Xi \psi|)=\operatorname{tr}(\rho \Xi \rho \Xi)=\operatorname{tr}\left(R^{2}\right)
$$

with

$$
R=\sqrt{\sqrt{\rho} \Xi \rho \Xi \sqrt{\rho}} .
$$

Here we have set $\rho=|\psi\rangle\langle\psi|$. The definition of the hermitian matrix $R$ however makes sense for arbitrary $\rho$ as well. If we write $\lambda_{j}, j=1, \ldots, 4$ for the eigenvalues of $R$ and $\lambda_{1}$ is without loss of generality the biggest one we can define the concurrence of an arbitrary two qubit state $\rho$ as 172

$$
C(\rho)=\max \left(0,2 \lambda_{1}-\operatorname{tr}(R)\right)=\max \left(0, \lambda_{1}-\lambda_{2}-\lambda_{3}-\lambda_{4}\right) .
$$

It is easy to see that $C(|\psi\rangle\langle\psi|)$ coincides with $C(\psi)$ from (5.17). The crucial point is now that Equation (5.15) holds for $E_{\mathrm{F}}(\rho)$ if we insert $C(\rho)$ instead of $C(\psi)$ :

Theorem 5.2.2 (Wootters Formula) The Entanglement of Formation of a two qubit system in a state $\rho$ is given by

$$
E_{\mathrm{F}}(\rho)=H\left[\frac{1}{2}\left(1+\sqrt{1-C(\rho)^{2}}\right)\right]
$$


where the concurrence of $\rho$ is given in Equation (5.2X) and $H$ denotes the binary entropy from (5.10).

To prove this theorem we have to find first a convex decomposition $\rho=$ $\sum_{j} \mu_{j}\left|\Psi_{j}\right\rangle\left\langle\Psi_{j}\right|$ of $\rho$ into pure states $\Psi_{j}$ such that the average reduced von Neumann entropy $\sum_{j} \mu_{j} E_{\mathrm{vN}}\left(\Psi_{j}\right)$ coincides with the right hand side of Equation (5.28). Second we have to show that we have really found the minimal decomposition. Since this is much more involved than the simple case discussed in Subsection 5.2.2 we omit the proof and refer to [172] instead. Note however that Equation (5.28) really coincides with the special cases we have derived for pure and Bell diagonal states. Finally let us add the remark that there is no analogon of Wootters' formula for higher dimensional Hilbert spaces. It can be shown [160] that the essential properties of the Bell basis $\Phi_{j}, j=0, . ., 3$ which would be necessary for such a generalization are available only in $2 \times 2$ dimensions.

5.2.4. Relative entropy for Bell diagonal states. - To calculate the Relative Entropy of Entanglement $E_{\mathrm{R}}$ for two qubit systems is more difficult. However there is at least an easy formula for Bell diagonal states which we will give in the following; [154.

Proposition 5.2.3 The Relative Entropy of Entanglement for a Bell diagonal state $\rho$ with highest eigenvalue $\lambda$ is given by (cf. Figure 5.1)

$$
E_{\mathrm{R}}(\rho)= \begin{cases}1-H(\lambda) & \lambda>\frac{1}{2} \\ 0 & \lambda \leq \frac{1}{2}\end{cases}
$$

Proof. For a Bell diagonal state $\rho=\sum_{j=0}^{3} \lambda_{j}\left|\Phi_{j}\right\rangle\left\langle\Phi_{j}\right|$ we have to calculate

$$
\begin{aligned}
E_{\mathrm{R}}(\rho)= & \inf _{\sigma \in \mathcal{D}}\left[\operatorname{tr}\left(\rho \log _{2} \rho-\rho \log _{2} \sigma\right)\right] \\
& =\operatorname{tr}\left(\rho \log _{2} \rho\right)+\inf _{\sigma \in \mathcal{D}}\left[-\sum_{j=0}^{3} \lambda_{j}\left\langle\Phi_{j}, \log _{2}(\sigma) \Phi_{j}\right\rangle\right] .
\end{aligned}
$$

Since $\log$ is a concave function we have $-\log _{2}\left\langle\Phi_{j}, \sigma \Phi_{j}\right\rangle \leq\left\langle\Phi_{j},-\log _{2}(\sigma) \Phi_{j}\right\rangle$ and therefore

$$
E_{\mathrm{R}}(\rho) \geq \operatorname{tr}\left(\rho \log _{2} \rho\right)+\inf _{\sigma \in \mathcal{D}}\left[-\sum_{j=0}^{3} \lambda_{j} \log _{2}\left\langle\Phi_{j}, \sigma \Phi_{j}\right\rangle\right] .
$$

Hence only the diagonal elements of $\sigma$ in the Bell basis enter the minimization on the right hand side of this inequality and this implies that we can restrict the infimum to the set of separable Bell diagonal state. Since a Bell diagonal state is separable iff all its eigenvalues are less than $1 / 2$ (Proposition 5.2.1) we get

$$
E_{\mathrm{R}}(\rho) \geq \operatorname{tr}\left(\rho \log _{2} \rho\right)+\inf _{p_{j} \in[0,1 / 2]}\left[-\sum_{j=0}^{3} \lambda_{j} \log _{2} p_{j}\right], \text { with } \sum_{j=0}^{3} p_{j}=1 .
$$

This is an optimization problem (with constraints) over only four real parameters and easy to solve. If the highest eigenvalue of $\rho$ is greater than $1 / 2$ we get $p_{1}=1 / 2$ and $p_{j}=\lambda_{j} /(2-2 \lambda)$, where we have chosen without loss of generality $\lambda=\lambda_{1}$. We get a lower bound on $E_{\mathrm{R}}(\rho)$ which is achieved if we insert the corresponding $\sigma$ in Equation (5.31). Hence we have proven the statement for $\lambda>1 / 2$. which completes the proof, since we have seen already that $\lambda \leq 1 / 2$ implies that $\rho$ is separable (Proposition 5.2.1). 


\subsection{Entanglement measures under symmetry}

The problems occuring if we try to calculate quantities like $E_{\mathrm{R}}$ or $E_{\mathrm{F}}$ for general density matrices arise from the fact that we have to solve optimization problems over very high dimensional spaces. One possible strategy to get explicit results is therefore parameter reduction by symmetry arguments. This can be done if the state in question admits some invariance properties like Werner, isotropic or OO-invariant states; cf. Section 3.1. We will give in the following some particular examples for such calculations, while a detailed discussion of the general idea (together with much more examples and further references) can be found in [159].

5.3.1. Entanglement of Formation. - Consider a compact group of unitaries $G \subset \mathcal{B}(\mathcal{H} \otimes \mathcal{H})$ (where $\mathcal{H}$ is again arbitrary finite dimensional), the set of $G$-invariant states, i.e. all $\rho$ with $[V, \rho]=0$ for all $V \in G$ and the corresponding twirl operation $P_{G} \sigma=\int_{G} V \sigma V^{*} d V$. Particular examples we are looking at are: 1 . Werner states where $G$ consists of all unitaries $U \otimes U$ 2. Isotropic states where each $V \in G$ has the form $V=U \otimes \bar{U}$ and finally 3. OO-invariant states where $G$ consists of unitaries $U \otimes U$ with real matrix elements $(U=\bar{U})$ and the twirl is given in Equation (3.24).

One way to calculate $E_{\mathrm{F}}$ for a $G$-invariant state $\rho$ consists now of the following steps: 1 . Determine the set $M_{\rho}$ of pure states $\Phi$ such that $P_{G}|\Phi\rangle\langle\Phi|=\rho$ holds. 2 . Calculate the function

$$
P_{G} \mathcal{S} \ni \rho \mapsto \epsilon_{G}(\rho)=\inf \left\{E_{\mathrm{vN}}(\sigma) \mid \sigma \in M_{\rho}\right\} \in \mathbb{R},
$$

where we have denoted the set of $G$-invariant states with $P_{G} \mathcal{S}$. 3. Determine $E_{\mathrm{F}}(\rho)$ then in terms of the convex hull of $\epsilon$, i.e.

$$
\begin{aligned}
& E_{\mathrm{F}}(\rho)=\inf \left\{\sum_{j} \lambda_{j} \epsilon\left(\sigma_{j}\right) \mid\right. \\
& \left.\qquad \sigma_{j} \in P_{G} \mathcal{S}, 0 \leq \lambda_{j} \leq 1, \quad \rho=\sum_{j} \lambda_{j} \sigma_{j}, \sum_{j} \lambda_{j}=1\right\} .
\end{aligned}
$$

The equality in the last Equation is of course a non-trivial statement which has to be proved. We skip this point, however, and refer the reader to [159]. The advantage of this scheme relies on the fact that spaces of $G$ invariant states are in general very low dimensional (if $G$ is not too small). Hence the optimization problem contained in step 3 has a much bigger chance to be tractable than the one we have to solve for the original definition of $E_{\mathrm{F}}$. There is of course no guarantee that any of this three steps can be carried out in a concrete situation. For the three examples mentioned above, however, there are results available, which we will present in the following.

5.3.2. Werner states. - Let us start with Werner states [159]. In this case $\rho$ is uniquely determined by its flip expectation value $\operatorname{tr}(\rho F)$ (cf. Subsection 3.1.2). To determine $\Phi \in \mathcal{H} \otimes \mathcal{H}$ such that $P_{\mathrm{UU}}|\Phi\rangle\langle\Phi|=\rho$ holds, we have to solve therefore the equation

$$
\langle\Phi, F \Phi\rangle=\sum_{j k} \Phi_{j k} \overline{\Phi_{k j}}=\operatorname{tr}(F \rho),
$$

where $\Phi_{j k}$ denote components of $\Phi$ in the canonical basis. On the other hand the reduced density matrix $\rho=\operatorname{tr}_{1}|\Phi\rangle\langle\Phi|$ has the matrix elements $\rho_{j k}=\sum_{l} \Phi_{j l} \Phi_{k l}$. By exploiting $U \otimes U$ invariance we can assume without loss of generality that $\rho$ is diagonal. Hence to get the function $\epsilon_{\mathrm{UU}}$ we have to minimize

$$
E_{\mathrm{vN}}(|\Phi\rangle\langle\Phi|)=\sum_{j} S\left[\sum_{k}\left|\Phi_{j k}\right|^{2}\right]
$$

under the constraint (5.36), where $S(x)=-x \log _{2}(x)$ denotes the von Neumann entropy. We skip these calculations here (see [159] instead) and state the results 


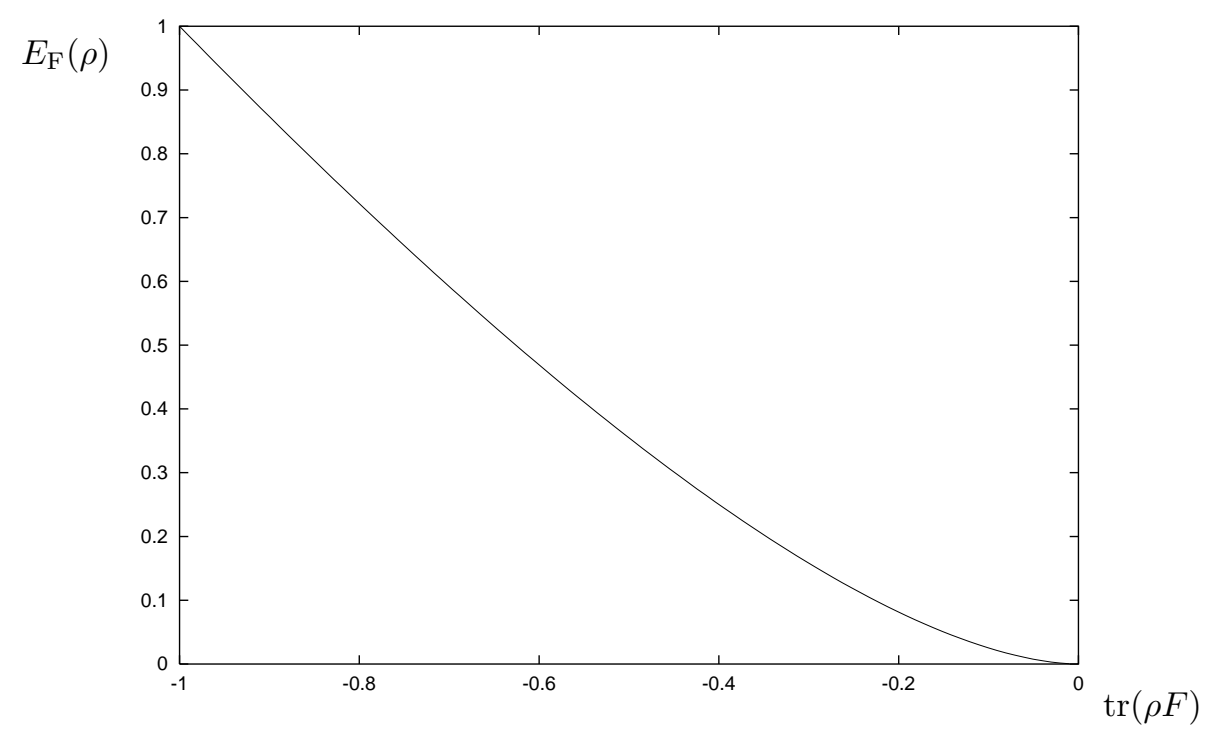

Figure 5.2: Entanglement of Formation for Werner states plotted as function of the flip expectation.

only. For $\operatorname{tr}(F \rho) \geq 0$ we get $\epsilon(\rho)=0$ (as expected since $\rho$ is separable in this case) and with $H$ from 5.16

$$
\epsilon_{\mathrm{UU}}(\rho)=H\left[\frac{1}{2}\left(1-\sqrt{1-\operatorname{tr}(F \rho)^{2}}\right)\right]
$$

for $\operatorname{tr}(F \rho)<0$. The minima are taken for $\Phi$ where all $\Phi_{j k}$ except one diagonal element are zero in the case $\operatorname{tr}(F \rho) \geq 0$ and for $\Phi$ with only two (non-diagonal) coefficients $\Phi_{j k}, \Phi_{k j}, j \neq k$ nonzero if $\operatorname{tr}(\rho F)<0$. The function $\epsilon$ is convex and coincides therefore with its convex hull such that we get

Proposition 5.3.1 For any Werner state $\rho$ the Entanglement of Formation is given by (cf. Figure 5.2)

$$
E_{\mathrm{F}}(\rho)= \begin{cases}H\left[\frac{1}{2}\left(1-\sqrt{1-\operatorname{tr}(F \rho)^{2}}\right)\right] & \operatorname{tr}(F \rho)<0 \\ 0 & \operatorname{tr}(F \rho) \geq 0\end{cases}
$$

5.3.3. Isotropic states. - Let us consider now isotropic, i.e. $U \otimes \bar{U}$ invariant states. They are determined by the expectation value $\operatorname{tr}(\rho \widetilde{F})$ with $\widetilde{F}$ from Equation (3.14). Hence we have to look first for pure states $\Phi$ with $\langle\Phi, \widetilde{F} \Phi\rangle=\operatorname{tr}(\rho \widetilde{F}$ ) (since this determines, as for Werner states above, those $\Phi$ with $\left.P_{\mathrm{UU}}(|\Phi\rangle\langle\Phi|)=\rho\right)$. To this end assume that $\Phi$ has the Schmidt decomposition $\Phi=\sum_{j} \lambda_{j} f_{j} \otimes f_{j}^{\prime}=U_{1} \otimes$ $U_{2} \sum_{j} \lambda_{j} e_{j} \otimes e_{j}$ with appropriate unitary matrices $U_{1}, U_{2}$ and the canonical basis 


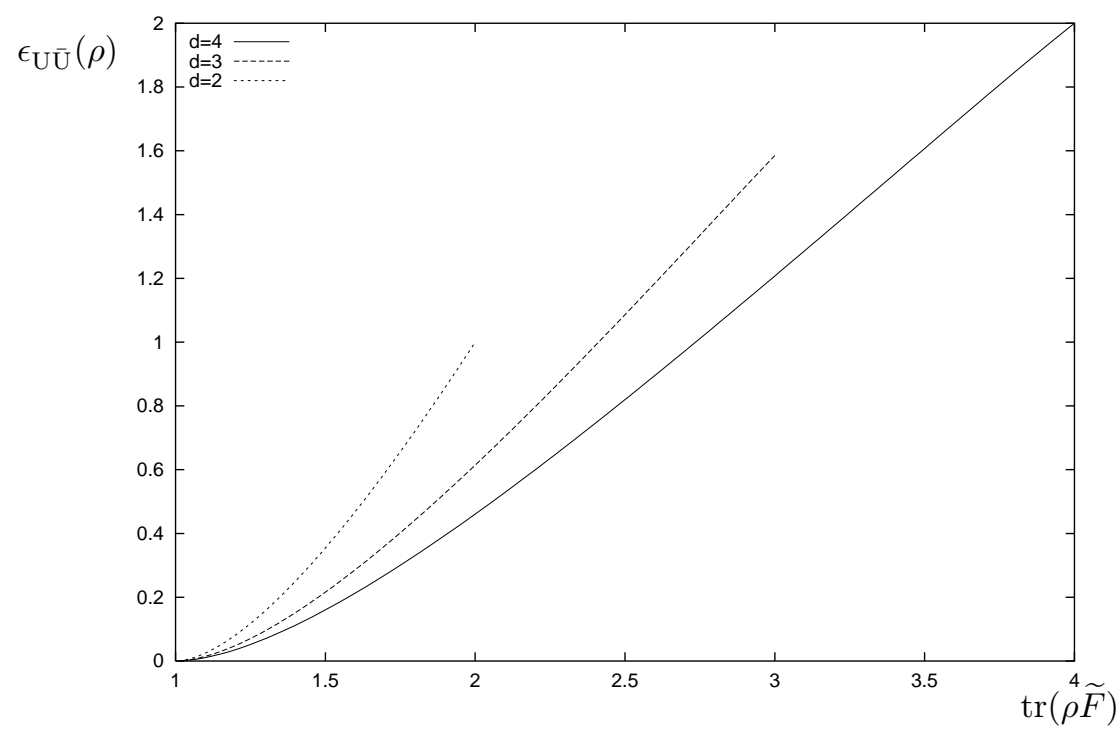

Figure 5.3: $\epsilon$-function for isotopic states plotted as a function of the flip expectation. For $d>2$ it is not convex near the right endpoint.

$e_{j}, j=1, \ldots, d$. Exploiting the $U \otimes \bar{U}$ invariance of $\rho$ we get

$$
\begin{aligned}
\operatorname{tr}(\rho \widetilde{F}) & =\left\langle(\mathbb{I} \otimes V) \sum_{j} \lambda_{j} e_{j} \otimes e_{j}, \widetilde{F}(\mathbb{I} \otimes V) \sum_{k} \lambda_{k} e_{k} \otimes e_{k}\right\rangle \\
& =\sum_{j, k, l, m} \lambda_{j} \lambda_{k}\left\langle e_{j} \otimes V e_{j}, e_{l} \otimes e_{l}\right\rangle\left\langle e_{m} \otimes e_{m}, e_{k} \otimes V e_{k}\right\rangle \\
& =\left|\sum_{j} \lambda_{j}\left\langle e_{j}, V e_{j}\right\rangle\right|^{2}
\end{aligned}
$$

with $V=U_{1}^{T} U_{2}$ and after inserting the definition of $\widetilde{F}$. Following our general scheme, we have to minimize $E_{\mathrm{vN}}(|\Phi\rangle\langle\Phi|)$ under the constraint given in Equation (5.42). This is explicitly done in 150. We will only state the result here, which leads to the function

$$
\epsilon_{\mathrm{U} \overline{\mathrm{U}}}(\rho)= \begin{cases}H(\gamma)+(1-\gamma) \log _{2}(d-1) & \operatorname{tr}(\rho \widetilde{F}) \geq \frac{1}{d} \\ 0 & \operatorname{tr}(\rho \widetilde{F})<0\end{cases}
$$

with

$$
\gamma=\frac{1}{d^{2}}(\sqrt{\operatorname{tr}(\rho \widetilde{F})}+\sqrt{[d-1][d-\operatorname{tr}(\rho \widetilde{F})]})^{2} .
$$

For $d \geq 3$ this function is not convex (cf. Figure 5.3), hence we get

Proposition 5.3.2 For any isotropic state the Entanglement of Formation is given as the convex hull

$$
E_{\mathrm{F}}(\rho)=\inf \left\{\sum_{j} \lambda_{j} \epsilon_{\mathrm{UU}}\left(\sigma_{j}\right) \mid \rho=\sum_{j} \lambda_{j} \sigma_{j}, P_{\mathrm{UU}} \sigma=\sigma\right\}
$$

of the function $\epsilon_{\mathrm{UU}}$ in Equation (5.43). 
5.3.4. OO-invariant states. - The results derived for isotropic and Werner states can be extended now to a large part of the set of OO-invariant states without solving new minimization problems. This is possible, because the definition of

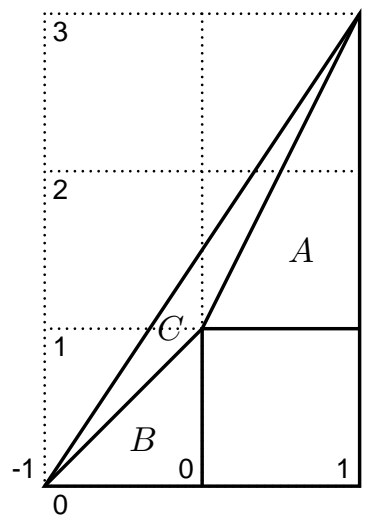

Figure 5.4: State space of OO-invariant states.
$E_{\mathrm{F}}$ in Equation (5.13) allows under some conditions an easy extension to a suitable set of non-symmetric states. If more precisely a nontrivial, minimizing decomposition $\rho=\sum_{j} p_{j}\left|\psi_{j}\right\rangle\left\langle\psi_{j}\right|$ of $\rho$ is known, all states $\rho^{\prime}$ which are a convex linear combination of the same $\left|\psi_{j}\right\rangle\left\langle\psi_{j}\right|$ but arbitrary $p_{j}^{\prime}$ have the same $E_{\mathrm{F}}$ as $\rho$ (see [159 for proof of the statement). For the general scheme we have presented in Subsection 5.3.1 this implies the following: If we know the pure states $\sigma \in M_{\rho}$ which solve the minimization problem for $\epsilon(\rho)$ in Equation (5.34) we get a minimizing decomposition of $\rho$ in terms of $U \in G$ translated copies of $\sigma$. This follows from the fact that $\rho$ is by definition of $M_{\rho}$ the twirl of $\sigma$. Hence any convex linear combination of pure states $U \sigma U^{*}$ with $U \in G$ has the same $E_{\mathrm{F}}$ as $\rho$.

A detailed analysis of the corresponding optimization problems in the case of Werner and isotropic states (which we have omitted here; see [159, 150] instead) leads therefore to the following results about OO-invariant states: The space of OO-invariant states decomposes into four regions: The separable square and three triangles $A, B, C$; cf. Figure 5.4. For all states $\rho$ in triangle $A$ we can calculate $E_{\mathrm{F}}(\rho)$ as for Werner states in Proposition 5.3.1 and in triangle $B$ we have to apply the result for isotropic states from Proposition 5.3.2. This implies in particular that $E_{\mathrm{F}}$ depends in $A$ only on $\operatorname{tr}(\rho F)$ and in $B$ only on $\operatorname{tr}(\rho \widetilde{F})$ and the dimension.

5.3.5. Relative Entropy of Entanglement. - To calculate $E_{\mathrm{R}}(\rho)$ for a symmetric state $\rho$ is even easier as the treatment of $E_{\mathrm{F}}(\rho)$, because we can restrict the minimization in the definition of $E_{\mathrm{R}}(\rho)$ in Equation (5.14) to $G$-invariant separable states, provided $G$ is a group of local unitaries. To see this assume that $\sigma \in \mathcal{D}$ minimizes $S(\rho \mid \sigma)$ for a $G$-invariant state $\rho$. Then we get $S\left(\rho \mid U \sigma U^{*}\right)=S(\rho \mid \sigma)$ for all $U \in G$ since the relative entropy $S$ is invariant under unitary transformations of both arguments and due to its convexity we even get $S\left(\rho \mid P_{G} \sigma\right) \leq S(\rho \mid \sigma)$. Hence $P_{G} \sigma$ minimizes $S(\rho \mid \cdot)$ as well, and since $P_{G} \sigma \in \mathcal{D}$ holds for a group $G$ of local unitaries, we get $E_{\mathrm{R}}(\sigma, \rho)=S\left(\rho \mid P_{G} \sigma\right)$ as stated. 


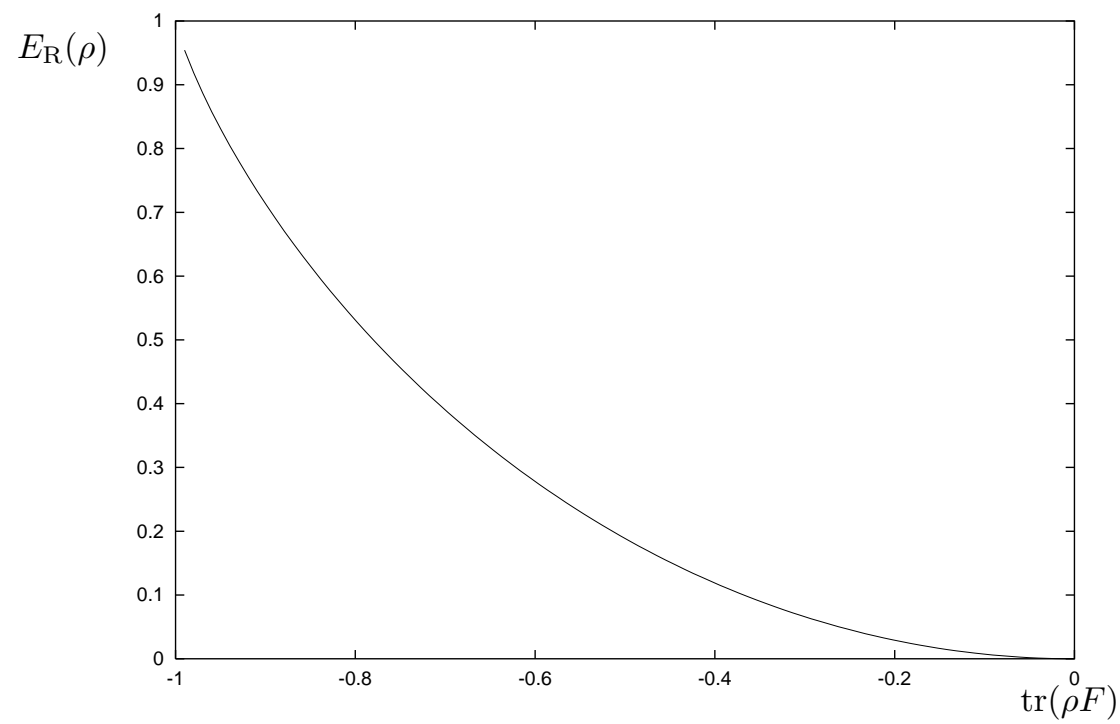

Figure 5.5: Relative Entropy of Entanglement for Werner states, plotted as a function of the flip expectation.

The sets of Werner and isotropic states are just intervals and the corresponding separable states form subintervals over which we have to perform the optimization. Due to the convexity of the relative entropy in both arguments, however, it is clear that the minimum is attained exactly at the boundary between entangled and separable states. For Werner states this is the state $\sigma_{0}$ with $\operatorname{tr}\left(F \sigma_{0}\right)=0$, i.e. it gives equal weight to both minimal projections. To get $E_{\mathrm{R}}(\rho)$ for a Werner state $\rho$ we have to calculate therefore only the relative entropy with respect to this state. Since all Werner states can be simultaneously diagonalized this is easily done and we get:

$$
E_{\mathrm{R}}(\rho)=1-H\left(\frac{1+\operatorname{tr}(F \rho)}{2}\right)
$$

Similarly, the boundary point $\sigma_{1}$ for isotropic states is given by $\operatorname{tr}\left(\widetilde{F} \sigma_{1}\right)=1$ which leads to

$$
E_{\mathrm{R}}(\rho)=\log _{2} d-\left(1-\frac{\operatorname{tr}(\widetilde{F} \rho)}{d}\right) \log _{2}(d-1)-S\left(\frac{\operatorname{tr}(\widetilde{F} \rho)}{d}, \frac{1-\operatorname{tr}(\widetilde{F} \rho)}{d}\right)
$$

for each entangled isotropic state $\rho$, and 0 if $\rho$ is separable. $\left(S\left(p_{1}, p_{2}\right)\right.$ denotes here the entropy of the probability vector $\left(p_{1}, p_{2}\right)$.) 


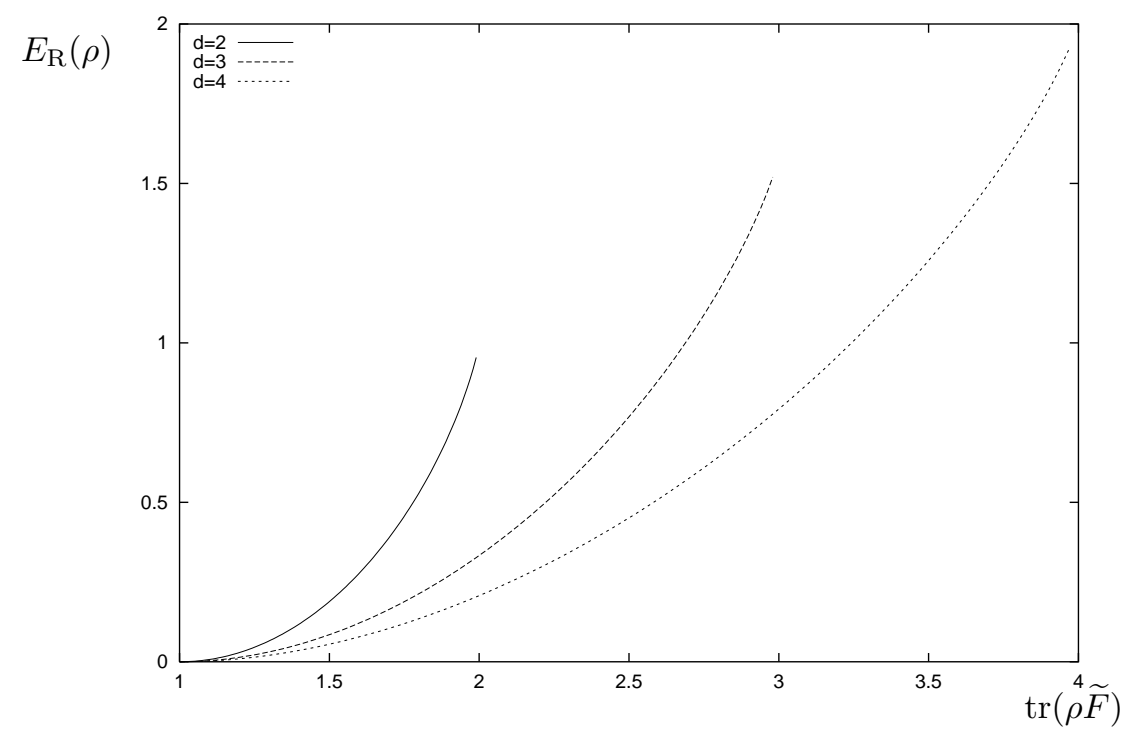

Figure 5.6: Relative Entropy of Entanglement for isotropic states and $d=2,3,4$, plotted as a function of $\operatorname{tr}(\rho \widetilde{F})$.

Let us consider now OO-invariant states. As for EOF we divide the state space into the separable square and the three triangles $A, B, C$; cf. Figure 5.4. The state at the coordinates $(1, d)$ is a maximally entangled state and all separable states on the line connecting $(0,1)$ with $(1,1)$ minimize the relative entropy for this state. Hence consider a particular state $\sigma$ on this line. The convexity property of the relative entropy shows immediately that $\sigma$ is a minimizer for all states on the line connecting $\sigma$ with the state at $(1, d)$. In this way it is easy to calculate $E_{\mathrm{R}}(\rho)$ for all $\rho$ in $A$. In a similar way we can treat the triangle $B$ : We just have to draw a line from $\rho$ to the state at $(-1,0)$ and find the minimizer for $\rho$ at the intersection with the separable border between $(0,0)$ and $(0,1)$. For all states in the triangle $C$ the relative entropy is minimized by the separable state at $(0,1)$.

An application of the scheme just reviewed is a proof that $E_{\mathrm{R}}$ is not additive, i.e. it does not satisfy Axiom E5b. To see this consider the state $\rho=\operatorname{tr}\left(P_{-}\right)^{-1} P_{-}$where $P_{-}$denotes the projector on the antisymmetric subspace. It is a Werner state with flip expectation -1 (i.e. it corresponds to the point $(-1,0)$ in Figure 5.4). According to our discussion above $S(\rho \mid \cdot)$ is minimized in this case by the separable state $\sigma_{0}$ and we get $E_{\mathrm{R}}(\rho)=1$ independently of the dimension $d$. The tensor product $\rho^{\otimes 2}$ can be regarded as a state in $\mathcal{S}\left(\mathcal{H}^{\otimes 2} \otimes \mathcal{H}^{\otimes 2}\right)$ with $U \otimes U \otimes V \otimes V$ symmetry, where $U, V$ are unitaries on $\mathcal{H}$. Note that the corresponding state space of $U U V V$ invariant states can be parameterized by the expectation of the three operators $F \otimes \mathbb{I}, \mathbb{I} \otimes F$ and $F \otimes F$ (cf. [159]) and we can apply the machinery just described to get the minimizer $\tilde{\sigma}$ of $S(\rho \mid \cdot)$. If $d>2$ holds it turns out that

$$
\widetilde{\sigma}=\frac{d+1}{2 d \operatorname{tr}\left(P_{+}\right)^{2}} P_{+} \otimes P_{+}+\frac{d-1}{2 d \operatorname{tr}\left(P_{-}\right)^{2}} P_{-} \otimes P_{-}
$$

holds (where $P_{ \pm}$denote the projections onto the symmetric and antisymmetric subspaces of $\mathcal{H} \otimes \mathcal{H}$ ) and not $\widetilde{\sigma}=\sigma_{0} \otimes \sigma_{0}$ as one would expect. As a consequence we get the inequality

$$
E_{\mathrm{R}}\left(\rho^{\otimes 2}\right)=2-\log _{2}\left(\frac{2 d-1}{d}\right)<2=S\left(\rho^{\otimes 2} \mid \sigma_{0}^{\otimes 2}\right)=2 E_{\mathrm{R}}(\rho) .
$$


$d=2$ is a special case, where $\sigma_{0}^{\otimes 2}$ and $\widetilde{\sigma}$ (and all their convex linear combination) give the same value 2. Hence for $d>2$ the Relative Entropy of Entanglement is, as stated, not additive. 


\section{Chapter 6}

\section{Channel capacity}

In Section 4.4 we have seen that it is possible to send (quantum) information undisturbed through a noisy quantum channel, if we encode one qubit into a (possibly long and highly entangled) string of qubits. This process is wasteful, since we have to use many instances of the channel to send just one qubit of quantum information. It is therefore natural to ask, which resources we need at least if we are using the best possible error correction scheme. More precisely the question is: With which maximal rate, i.e. information sent per channel usage, we can transmit quantum information undisturbed through a noisy channel? This question naturally leads to the concept of channel capacities which we will review in this chapter.

\subsection{The general case}

We are mainly interested in classical and quantum capacities. The basic ideas behind both situations are however quite similar. In this section we will consider therefore a general definition of capacity which applies to arbitrary channels and both kinds of information. (See also 169 as a general reference for this section.)

6.1.1. The definition. - Hence consider two observable algebras $\mathcal{A}_{1}, \mathcal{A}_{2}$ and an arbitrary channel $T: \mathcal{A}_{1} \rightarrow \mathcal{A}_{2}$. To send systems described by a third observable algebra $\mathcal{B}$ undisturbed through $T$ we need an encoding channel $E: \mathcal{A}_{2} \rightarrow \mathcal{B}$ and a decoding channel $D: \mathcal{B} \rightarrow \mathcal{A}_{1}$ such that ETD equals the ideal channel $\mathcal{B} \rightarrow \mathcal{B}$, i.e. the identity on $\mathcal{B}$. Note that the algebra $\mathcal{B}$ describing the systems to send, and the input respectively output algebra of $T$ need not to be of the same type, e.g. $\mathcal{B}$ can be classical while $\mathcal{A}_{1}, \mathcal{A}_{2}$ are quantum (or vice versa).

In general (i.e. for arbitrary $T$ and $\mathcal{B}$ ) it is of course impossible to find such a pair $E$ and $D$. In this case we are interested at least in encodings and decodings which make the error produced during the transmission as small as possible. To make this statement precise we need a measure for this error and there are in fact many good choices for such a quantity (all of them leading to equivalent results, cf. Subsection 6.3.1). We will use in the following the "cb-norm difference" $\| E T D$ - Id $\|_{\mathrm{cb}}$, where Id is the identity (i.e. ideal) channel on $\mathcal{B}$ and $\|\cdot\|_{\mathrm{cb}}$ denotes the norm of complete boundedness ("cb-norm" for short)

$$
\|T\|_{\mathrm{cb}}=\sup _{n \in \mathbb{N}}\left\|T \otimes \operatorname{Id}_{n}\right\|, \quad \operatorname{Id}_{n}: \mathcal{B}\left(\mathbb{C}^{n}\right) \rightarrow \mathbb{B}\left(\mathbb{C}^{n}\right)
$$

The cb-norm improves the sometimes annoying property of the usual operator norm that quantities like $\left\|T \otimes \operatorname{Id}_{\mathcal{B}\left(\mathbb{C}^{d}\right)}\right\|$ may increase with the dimension $d$. On infinite dimensional observable algebras $\|T\|_{\mathrm{cb}}$ can be infinite although each term in the supremum is finite. A particular example for a map with such a behavior is the transposition on an infinite dimensional Hilbert space. A map with finite cb-norm is therefore called completely bounded. In a finite dimensional setup each linear map is completely bounded. For the transposition $\Theta$ on $\mathbb{C}^{d}$ we have in particular $\|\Theta\|_{\mathrm{cb}}=d$. The cb-norm has some nice features which we will use frequently; this includes its multiplicativity $\left\|T_{1} \otimes T_{2}\right\|_{\mathrm{cb}}=\left\|T_{1}\right\|_{\mathrm{cb}}\left\|T_{2}\right\|_{\mathrm{cb}}$ and the fact that $\|T\|_{\mathrm{cb}}=1$ holds for each (unital) channel. Another useful relation is $\|T\|_{\mathrm{cb}}=\left\|T \otimes \operatorname{Id}_{\mathcal{B}(\mathcal{H})}\right\|$, which holds if $T$ is a map $\mathcal{B}(\mathcal{H}) \rightarrow \mathcal{B}(\mathcal{H})$. For more properties of the cb-norm let us refer to 125 .

Now we can define the quantity

$$
\Delta(T, \mathcal{B})=\inf _{E, D}\left\|E T D-\operatorname{Id}_{\mathcal{B}}\right\|_{\mathrm{cb}}
$$


where the infimum is taken over all channels $E: \mathcal{A}_{2} \rightarrow \mathcal{B}$ and $D: \mathcal{B} \rightarrow \mathcal{A}_{1}$ and $\operatorname{Id}_{\mathcal{B}}$ is again the ideal $\mathcal{B}$-channel. $\Delta$ describes, as indicated above, the smallest possible error we have to take into account if we try to transmit one $\mathcal{B}$ system through one copy of the channel $T$ using any encoding $E$ and decoding $D$. In Section 4.4, however, we have seen that we can reduce the error if we take $M$ copies of the channel instead of just one. More generally we are interested in the transmission of "codewords of length" $N$, i.e. $\mathcal{B}^{\otimes N}$ systems using $M$ copies of the channel $T$. Encodings and decodings are in this case channels of the form $E: \mathcal{A}_{2}^{\otimes M} \rightarrow \mathcal{B}^{\otimes N}$ respectively $D: \mathcal{B}^{\otimes N} \rightarrow \mathcal{A}_{1}^{\otimes M}$. If we increase the number $M$ of channels the error $\Delta\left(T^{\otimes M}, \mathcal{B}^{\otimes N(M)}\right)$ decreases provided the rate with which $N$ grows as a function of $M$ is not too large. A more precise formulation of this idea leads to the following definition.

Definition 6.1.1 Let $T$ be a channel and $\mathcal{B}$ an observable algebra. A number $c \geq 0$ is called achievable rate for $T$ with respect to $\mathcal{B}$, if for any pair of sequences $M_{j}, N_{j}$, $j \in \mathbb{N}$ with $M_{j} \rightarrow \infty$ and $\limsup _{j \rightarrow \infty} N_{j} / M_{j}<c$ we have

$$
\lim _{j \rightarrow \infty} \Delta\left(T^{\otimes M_{j}}, \mathcal{B}^{\otimes N_{j}}\right)=0
$$

The supremum of all achievable rates is called the capacity of $T$ with respect to $\mathcal{B}$ and denoted by $C(T, \mathcal{B})$.

Note that by definition $c=0$ is an achievable rate hence $C(T, \mathcal{B}) \geq 0$. If on the other hand each $c>0$ is achievable we write $C(T, \mathcal{B})=\infty$. At a first look it seems cumbersome to check all pairs of sequences with given upper ratio when testing $c$. Due to some monotonicity properties of $\Delta$, however, it can be shown that it is sufficient to check only one sequence provided the $M_{j}$ satisfy the additional condition $M_{j} /\left(M_{j+1}\right) \rightarrow 1$.

6.1.2. Simple calculations. - We see that there are in fact many different capacities of a given channel depending on the type of information we want to transmit. However, there are only two different cases we are interested in: $\mathcal{B}$ can be either classical or quantum. We will discuss both special cases in greater detail in the next two sections. Before we do this, however, we will have a short look on some simple calculations which can be done in the general case. To this end it is convenient to introduce the notations

$$
\mathcal{M}_{d}=\mathcal{B}\left(\mathbb{C}^{d}\right) \quad \text { and } \quad \mathcal{C}_{d}=\mathcal{C}(\{1, \ldots, d\})
$$

as shorthand notations for $\mathcal{B}\left(\mathbb{C}^{d}\right)$ and $\mathcal{C}(\{1, \ldots, d\})$ since some notations become otherwise a little bit clumsy. First of all let us have a look on capacities of ideal channels. If $\operatorname{Id}_{\mathcal{M}_{f}}$ and $\operatorname{Id}_{\mathcal{C}_{f}}$ denote the identity channels on the quantum algebra $\mathcal{M}_{f}$ respectively the classical algebra $\mathcal{C}_{f}$ we get

$$
C\left(\operatorname{Id}_{\mathcal{C}_{f}}, \mathcal{M}_{d}\right)=0, C\left(\operatorname{Id}_{\mathcal{C}_{f}}, \mathrm{C}_{d}\right)=C\left(\operatorname{Id}_{\mathcal{M}_{f}}, \mathcal{M}_{d}\right)=C\left(\operatorname{Id}_{M_{f}}, \mathcal{C}_{d}\right)=\frac{\log _{2} f}{\log _{2} d}
$$

The first equation is the channel capacity version of the no-teleportation theorem: It is impossible to transfer quantum information through a classical channel. The other equations follow simply by counting dimensions.

For the next relation it is convenient to associate to a pair of channels $T, S$ the quantity $C(T, S)$ which arises if we replace in Definition 6.1.1 and Equation (6.2) the ideal channel $\operatorname{Id}_{\mathcal{B}}$ by an arbitrary channel $S$. Hence $C(T, S)$ is a slight generalization of the channel capacity which describes with which asymptotic rate the channel $S$ can be approximated by $T$ (and appropriate encodings and decodings). 
These generalized capacities satisfy the two step coding inequality, i.e. for the three channels $T_{1}, T_{2}, T_{3}$ we have

$$
C\left(T_{3}, T_{1}\right) \geq C\left(T_{2}, T_{1}\right) C\left(T_{3}, T_{2}\right)
$$

To prove it consider the relations

$$
\begin{aligned}
\| T_{1}^{\otimes N} & -E_{1} E_{2} T_{3}^{\otimes K} D_{2} D_{1} \|_{\mathrm{cb}} \\
& =\left\|T_{1}^{\otimes N}-E_{1} T_{2}^{\otimes M} D_{1}+E_{1} T_{2}^{\otimes M} D_{1}-E_{1} E_{2} T_{3}^{\otimes K} D_{2} D_{1}\right\|_{\mathrm{cb}} \\
& \leq\left\|T_{1}^{\otimes N}-E_{1} T_{2}^{\otimes M} D_{1}\right\|_{\mathrm{cb}}+\left\|E_{1}\right\|_{\mathrm{cb}}\left\|T_{2}^{\otimes M}-E_{2} T_{3}^{\otimes K} D_{2}\right\|_{\mathrm{cb}}\left\|D_{1}\right\|_{\mathrm{cb}} \\
& \leq\left\|T_{1}^{\otimes N}-E_{1} T_{2}^{\otimes M} D_{1}\right\|_{\mathrm{cb}}+\left\|T_{2}^{\otimes M}-E_{2} T_{3}^{\otimes K} D_{2}\right\|_{\mathrm{cb}}
\end{aligned}
$$

where we have used for the last inequality the fact that the cb-norm of a channel is one. If $c_{1}$ is an achievable rate of $T_{1}$ with respect to $T_{2}$ such that $\limsup _{j \rightarrow \infty} M_{j} / N_{j}<c_{1}$ and $c_{2}$ is an achievable rate of $T_{2}$ with respect to $T_{3}$ such that $\limsup _{j \rightarrow \infty} N_{j} / K_{j}<c_{2}$ we see that

$$
\limsup _{j \rightarrow \infty} \frac{M_{j}}{K_{j}}=\limsup _{j \rightarrow \infty} \frac{M_{j}}{N_{j}} \frac{N_{j}}{K_{j}} \leq \limsup _{j \rightarrow \infty} \frac{M_{j}}{N_{j}} \limsup _{k \rightarrow \infty} \frac{N_{k}}{K_{k}} .
$$

If we choose the sequences $M_{j}, N_{j}$ and $K_{j}$ clever enough (cf. the remark following Definition 6.1.1) this implies that $c_{1} c_{2}$ is an achievable rate for $T_{1}$ with respect to $T_{3}$ and this proves Equation (6.6).

As a first application of (6.6), we can relate all capacities $C\left(T, \mathcal{M}_{d}\right)$ (and $\left.C\left(T, \mathrm{C}_{d}\right)\right)$ for different $d$ to one another. If we choose $T_{3}=T, T_{1}=\operatorname{Id}_{\mathcal{M}_{d}}$ and $T_{2}=\operatorname{Id}_{\mathcal{M}_{f}}$ we get with 6.5) $C\left(T, \mathcal{M}_{d}\right) \leq \frac{\log _{2} f}{\log _{2} d} C\left(T, \mathcal{M}_{f}\right)$, and exchanging $d$ with $f$ shows that even equality holds. A similar relation can be shown for $C\left(T, \mathrm{e}_{d}\right)$. Hence the dimension of the observable algebra $\mathcal{B}$ describing the type of information to be transmitted, enters only via a multiplicative constant, i.e. it is only a choice of units and we define the classical capacity $C_{c}(T)$ and the quantum capacity $C_{q}(T)$ of a channel $T$ as

$$
C_{c}(T)=C\left(T, \mathrm{C}_{2}\right), \quad C_{q}(T)=C\left(T, \mathcal{M}_{2}\right)
$$

A second application of Equation (6.6) is a relation between the classical and the quantum capacity of a channel. Setting $T_{3}=T, T_{1}=\operatorname{Id}_{\mathcal{C}_{2}}$ and $T_{2}=\operatorname{Id}_{\mathcal{M}_{2}}$ we get again with 6.5

$$
C_{q}(T) \leq C_{c}(T)
$$

Note that it is now not possible to interchange the roles of $\mathcal{C}_{2}$ and $\mathcal{M}_{2}$. Hence equality does not hold here.

Another useful relation concerns concatenated channels: We transmit information of type $\mathcal{B}$ first through a channel $T_{1}$ and then through a second channel $T_{2}$. It is reasonable to assume that the capacity of the composition $T_{2} T_{1}$ can not be bigger than capacity of the channel with the smallest bandwidth. This conjecture is indeed true and known as the "Bottleneck inequality":

$$
C\left(T_{2} T_{1}, \mathcal{B}\right) \leq \min \left\{C\left(T_{1}, \mathcal{B}\right), C\left(T_{2}, \mathcal{B}\right)\right\}
$$

To see this consider an encoding and a decoding channel $E$ respectively $D$ for $\left(T_{2} T_{1}\right)^{\otimes M}$, i.e. in the definition of $C\left(T_{2} T_{1}, \mathcal{B}\right)$ we look at

$$
\left\|\mathrm{Id}_{\mathcal{B}}^{\otimes N}-E\left(T_{2} T_{1}\right)^{\otimes M} D\right\|_{\mathrm{cb}}=\left\|\operatorname{Id}_{\mathcal{B}}^{\otimes N}-\left(E T_{2}^{\otimes M}\right) T_{1}^{\otimes M} D\right\|_{\mathrm{cb}}
$$


This implies that $E T_{2}^{\otimes M}$ and $D$ are an encoding and a decoding channel for $T_{1}$. Something similar holds for $D$ and $T_{1}^{\otimes M} D$ with respect to $T_{2}$. Hence each achievable rate for $T_{2} T_{1}$ is also an achievable rate for $T_{2}$ and $T_{1}$, and this proves Equation (6.13).

Finally we want to consider two channels $T_{1}, T_{2}$ in parallel, i.e. we consider the tensor product $T_{1} \otimes T_{2}$. If $E_{j}, D_{j}, j=1,2$ are encoding, respectively decoding channels for $T_{1}^{\otimes M}$ and $T_{2}^{\otimes M}$ such that $\left\|\operatorname{Id}_{\mathcal{B}}^{\otimes N_{j}}-E_{j} T_{j}^{\otimes M} D_{j}\right\|_{\mathrm{cb}} \leq \epsilon$ holds, we get

$$
\begin{aligned}
\| \mathrm{Id}- & \mathrm{Id} \otimes\left(E_{2} T^{\otimes M} D_{2}\right)+\mathrm{Id} \otimes\left(E_{2} T^{\otimes M} D_{2}\right)-E_{1} \otimes E_{2}\left(T_{1} \otimes T_{2}\right)^{\otimes M} D_{1} \otimes D_{2} \|_{\mathrm{cb}} \\
& \leq \| \mathrm{Id} \otimes\left(\mathrm{Id}-E_{2} T^{\otimes M} D_{2}\left\|_{\mathrm{cb}}+\right\|\left(\mathrm{Id}-E_{1} T_{1}^{\otimes M} D_{1}\right) \otimes E_{2} T^{\otimes M} D_{2} \|_{\mathrm{cb}}\right. \\
& \leq\left\|\mathrm{Id}-E_{2} T^{\otimes M} D_{2}\right\|_{\mathrm{cb}}+\left\|\mathrm{Id}-E_{1} T_{1}^{\otimes M} D_{1}\right\|_{\mathrm{cb}} \leq 2 \epsilon
\end{aligned}
$$

Hence $c_{1}+c_{2}$ is achievable for $T_{1} \otimes T_{2}$ if $c_{j}$ is achievable for $T_{j}$. This implies the inequality

$$
C\left(T_{1} \otimes T_{2}, \mathcal{B}\right) \geq C\left(T_{1}, \mathcal{B}\right)+C\left(T_{2}, \mathcal{B}\right) .
$$

When all channels are ideal, or when all systems involved are classical even equality holds, i.e. channel capacities are additive in this case. However, if quantum channels are considered, it is one of the big open problems of the field, to decide under which conditions additivity holds.

\subsection{The classical capacity}

In this section we will discuss the classical capacity $C_{c}(T)$ of a channel $T$. There are in fact three different cases to consider: $T$ can be either classical or quantum and in the quantum case we can use either ordinary encodings and decodings or a dense coding scheme (cf. Subsection 4.1.3).

6.2.1. Classical channels. - Let us consider first a classical to classical channel $T: \mathcal{C}(Y) \rightarrow \mathcal{C}(X)$. This is basically the situation of classical information theory and we will only have a short look here - mainly to show how this (well known) situation fits into the general scheme described in the last section!.

First of all we have to calculate the error quantity $\Delta\left(T, \mathcal{C}_{2}\right)$ defined in Equation (6.2). As stated in Subsection 3.2.3 $T$ is completely determined by its transition probabilities $T_{x y},(x, y) \in X \times Y$ describing the probability to receive $x \in X$ when $y \in Y$ was sent. Since the cb-norm for a classical algebra coincides with the ordinary norm we get (we have set $X=Y$ for this calculation):

$$
\begin{aligned}
\|\mathrm{Id}-T\|_{\mathrm{cb}} & =\|\mathrm{Id}-T\|=\sup _{x, f}\left|\sum_{y}\left(\delta_{x y}-T_{x y}\right) f_{y}\right| \\
& =2 \sup _{x}\left(1-T_{x x}\right)
\end{aligned}
$$

where the supremum in the first equation is taken over all $f \in \mathfrak{C}(X)$ with $\|f\|=$ $\sup _{y}\left|f_{y}\right| \leq 1$. We see that the quantity in Equation (6.20) is exactly twice the maximal error probability, i.e. the maximal probability of sending $x$ and getting anything different. Inserting this quantity for $\Delta$ in Definition 6.1.1 applied to a classical channel $T$ and the "bit-algebra" $\mathcal{B}=\mathcal{C}_{2}$, we get exactly Shannons classical definition of the capacity of a discrete memoryless channel 138 .

Hence we can apply Shannons noisy channel coding theorem to calculate $C_{c}(T)$ for a classical channel. To state it we have to introduce first some terminology. Consider therefore a state $p \in \mathcal{C}^{*}(X)$ of the classical input algebra $\mathrm{C}(X)$ and its

\footnotetext{
${ }^{1}$ Please note that this implies in particular that we do got give a complete review of the foundations of classical information theory here; cf 101, 62, 49. instead.
} 
image $q=T^{*}(p) \in \mathfrak{C}^{*}(Y)$ under the channel. $p$ and $q$ are probability distributions on $X$ respectively $Y$ and $p_{x}$ can be interpreted as the probability that the "letter" $x \in X$ was send. Similarly $q_{y}=\sum_{x} T_{x y} p_{x}$ is the probability that $y \in Y$ was received and $P_{x y}=T_{x y} p_{x}$ is the probability that $x \in X$ was sent and $y \in Y$ was received. The family of all $P_{x y}$ can be interpreted as a probability distribution $P$ on $X \times Y$ and the $T_{x y}$ can be regarded as conditional probability of $P$ under the condition $x$. Now we can introduce the mutual information

$$
I(p, T)=S(p)+S(q)-S(P)=\sum_{(x, y) \in X \times Y} P_{x y} \log _{2}\left(\frac{P_{x y}}{p_{x} q_{y}}\right),
$$

where $S(p), S(q)$ and $S(P)$ denote the entropies of $p, q$ and $P$. The mutual information describes, roughly speaking, the information that $p$ and $q$ contain about each other. E.g. if $p$ and $q$ are completely uncorrelated (i.e. $P_{x y}=p_{x} q_{y}$ ) we get $I(p, T)=0$. If $T$ is on the other hand an ideal bit-channel and $p$ equally distributed we have $I(p, T)=1$. Now we can state Shannons Theorem which expresses the classical capacity of $T$ in terms of mutual informations [138]:

TheOREm 6.2 .1 (Shannon) The classical capacity of $C_{c}(T)$ of a classical communication channel $T: \mathcal{C}(Y) \rightarrow \mathcal{C}(X)$ is given by

$$
C_{c}(T)=\sup _{p} I(p, T),
$$

where the supremum is taken over all states $p \in \mathcal{C}^{*}(X)$.

6.2.2. Quantum channels. - If we transmit classical data through a quantum channel $T: \mathcal{B}(\mathcal{H}) \rightarrow \mathcal{B}(\mathcal{H})$ the encoding $E: \mathcal{B}(\mathcal{H}) \rightarrow \mathcal{C}_{2}$ is a parameter dependent preparation and the decoding $D: \mathcal{C}_{2} \rightarrow \mathcal{B}(\mathcal{H})$ is an observable. Hence the composition ETD is a channel $\mathcal{C}_{2} \rightarrow \mathcal{C}_{2}$, i.e. a purely classical channel and we can calculate its capacity in terms of Shannons Theorem (Theorem 6.2.1). This observation leads to the definition of the "one-shot" classical capacity of $T$ :

$$
C_{c, 1}(T)=\sup _{E, D} C_{c}(E T D)
$$

where the supremum is taken over all encodings and decodings of classical bits. The term "one-shot" in this definition arises from the fact that we need apparently only one invocation of the channel $T$. However many uses of the channel are hidden in the definition of the classical capacity on the right hand side. Hence $C_{c, 1}(T)$ can be defined alternatively in the same way as $C_{c}(T)$ except that no entanglement is allowed during encoding and decoding, or more precisely in Definition 6.1.1 we consider only encodings $E: \mathcal{B}(\mathcal{K})^{\otimes M} \rightarrow \mathcal{C}_{2}^{\otimes N}$ which prepare separable states and only decodings $D: \mathcal{C}_{2}^{\otimes N} \rightarrow \mathcal{B}(\mathcal{H})^{\otimes M}$ which lead to separable observables. It is not yet known, whether entangled codings can help to increase the transmission rate. Therefore we only know that

$$
C_{c, 1}(T) \leq C_{c}(T)=\sup _{M \in \mathbb{N}} \frac{1}{M} C_{c, 1}\left(T^{\otimes M}\right)
$$

holds. One reason why $C_{c, 1}(T)$ is an interesting quantity relies on the fact that we have, due to the following theorem by Holevo [80] a computable expression for it.

THEOREM 6.2.2 The one-shot classical capacity $C_{c, 1}(T)$ of a quantum channel $T$ : $\mathcal{B}(\mathcal{H}) \rightarrow \mathcal{B}(\mathcal{H})$ is given by

$$
C_{c, 1}(T)=\sup _{p_{j}, \rho_{j}}\left[S\left(\sum_{j} p_{j} T^{*}\left[\rho_{j}\right]\right)-\sum_{j} p_{j} S\left(T^{*}\left[\rho_{j}\right]\right)\right],
$$


where the supremum is taken over all probability distributions $p_{j}$ and collections of density operators $\rho_{j}$.

6.2.3. Entanglement assisted capacity. - Another classical capacity of a quantum channel arises, if we use dense coding schemes instead of simple encodings and decodings to transmit the data through the channel $T$. In other words we can define the entanglement enhanced classical capacity $C_{e}(T)$ in the same way as $C_{c}(T)$ but by replacing the encoding and decoding channels in Definition 6.1.1 and Equation (6.2) by dense coding protocols. Note that this implies that the sender Alice and the receiver Bob share an (arbitrary) amount of (maximally) entangled states prior to the transmission.

For this quantity a coding theorem was proven recently by Bennett and others [18] which we want to state in the following. To this end assume that we are transmitting systems in the state $\rho \in \mathcal{B}^{*}(\mathcal{H})$ through the channel and that $\rho$ has the purification $\Psi \in \mathcal{H} \otimes \mathcal{H}$, i.e. $\rho=\operatorname{tr}_{1}|\Psi\rangle\left\langle\Psi\left|=\operatorname{tr}_{2}\right| \Psi\right\rangle\langle\Psi|$. Then we can define the entropy exchange

$$
S(\rho, T)=S[(T \otimes \operatorname{Id})(|\Psi\rangle\langle\Psi|)]
$$

The density operator $(T \otimes \mathrm{Id})(|\Psi\rangle\langle\Psi|)$ has the output state $T^{*}(\rho)$ and the input state $\rho$ as its partial traces. It can be regarded therefore as the quantum analog of the input/output probability distribution $T_{x y}$ defined in Subsection 6.2.1. Another way to look at $S(\rho, T)$ is in terms of an ancilla representation of $T$ : If $T^{*}(\rho)=$ $\operatorname{tr}_{\mathcal{K}}\left(U \rho \otimes \rho_{\mathcal{K}} U^{*}\right)$ with a unitary $U: \mathcal{H} \otimes \mathcal{K}$ and a pure environment state $\rho_{\mathcal{K}}$ it can be shown [7] that $S(\rho, T)=S\left[T_{\mathcal{K}}^{*} \rho\right]$ where $T_{\mathcal{K}}$ is the channel describing the information transfer into the environment, i.e. $T_{\mathcal{K}}^{*}(\rho)=\operatorname{tr}_{\mathcal{H}}\left(U \rho \otimes \rho_{\mathcal{K}} U^{*}\right)$, in other words $S(\rho, T)$ is the final entropy of the environment. Now we can define

$$
I(\rho, T)=S(\rho)+S\left(T^{*} \rho\right)-S(\rho, T)
$$

which is the quantum analog of the mutual information given in Equation (6.21). It has a number of nice properties, in particular positivity, concavity with respect to the input state and additivity [2] and its maximum with respect to $\rho$ coincides actually with $C_{e}(T)$ [18].

THEOREM 6.2.3 The entanglement assisted capacity $C_{e}(T)$ of a quantum channel $T: \mathcal{B}(\mathcal{H}) \rightarrow \mathcal{B}(\mathcal{H})$ is given by

$$
C_{e}(T)=\sup _{\rho} I(\rho, T)
$$

where the supremum is taken over all input states $\rho \in \mathcal{B}^{*}(\mathcal{H})$.

Due to the nice additivity properties of the quantum mutual information $I(\rho, T)$ the capacity $C_{e}(T)$ is known to be additive as well. This implies that it coincides with the corresponding "one-shot" capacity, and this is an essential simplification compared to the classical capacity $C_{c}(T)$.

6.2.4. Examples. - Although the expressions in Theorem 6.2.2 and 6.2.3 are much easier then the original definitions they involve still some optimization problems over possibly large parameter spaces. Nevertheless there are special cases which allow explicit calculations. As a first example we will consider the "quantum erasure channel" which transmits with probability $1-\vartheta$ the $d$-dimensional input state intact while it is replaced with probability $\vartheta$ by an "erasure symbol", i.e. a $(d+1)^{\text {th }}$ pure state $\psi_{e}$ which is orthogonal to all others [72]. In the Schrödinger picture this is

$$
\mathcal{B}^{*}\left(\mathbb{C}^{d}\right) \ni \rho \mapsto T^{*}(\rho)=(1-\vartheta) \rho+\vartheta \operatorname{tr}(\rho)\left|\psi_{e}\right\rangle\left\langle\psi_{e}\right| \in \mathcal{B}^{*}\left(\mathbb{C}^{d+1}\right) .
$$


This example is very unusal, because all capacities discussed up to now (including the quantum capacity as we will see in Subsection 6.3.2) can be calculated explicitly: We get $C_{c, 1}(T)=C_{c}(T)=(1-\vartheta) \log _{2}(d)$ for the classical and $C_{e}(T)=2 C_{c}(T)$ for the entanglement enhanced classical capacity 115, 17]. Hence the gain by entanglement assistance is exactly a factor two; cf. Figure 6.1.

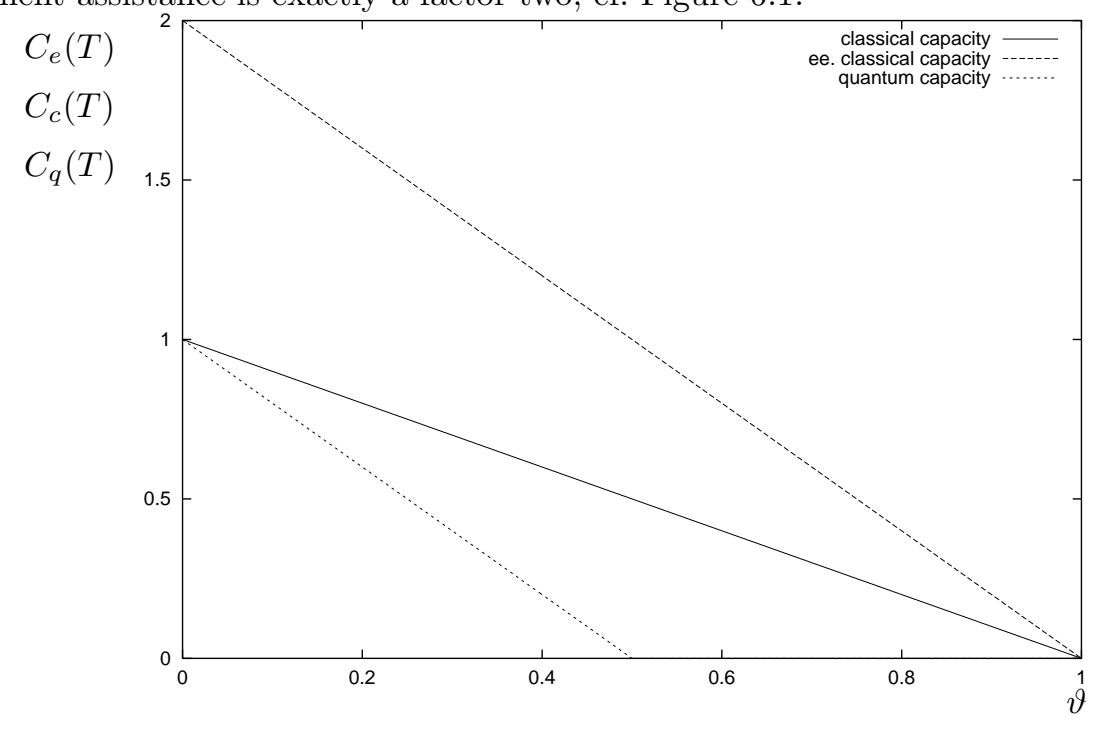

Figure 6.1: Capacities of the quantum erasure channel plotted as a function of the error probability.

Our next example is the depolarizing channel

$$
\mathcal{B}^{*}\left(\mathbb{C}^{d}\right) \ni \rho \mapsto T^{*}(\rho)=(1-\vartheta) \rho+\vartheta \operatorname{tr}(\rho) \frac{\mathbb{I}}{d} \in \mathcal{B}^{*}\left(\mathbb{C}^{d}\right),
$$

already discussed in Section 3.2. It is more interesting and more difficult to study. It is in particular not known whether $C_{c}$ and $C_{c, 1}$ coincide in this case (i.e. the value of $C_{c}$ is not known. Therefore we can compare $C_{e}(T)$ only with with $C_{c, 1}$. Using the unitary covariance of $T$ (cf. Subsection 3.2.2) we see first that $I\left(U \rho U^{*}, T\right)=I(\rho, T)$ holds for all unitaries $U$ (to calculate $S\left(U \rho U^{*}, T\right)$ note that $U \otimes U \Psi$ is a purification of $U \rho U^{*}$ if $\Psi$ is a purification of $\rho$ ). Due to the concavity of $I(\rho, T)$ in the first argument we can average over all unitaries and see that the maximum in Equation (6.28) is achieved on the maximally mixed state. Straightforward calculation therefore shows that

$$
C_{e}(T)=\log _{2}\left(d^{2}\right)+\left(1-\vartheta \frac{d^{2}-1}{d^{2}}\right) \log _{2}\left(1-\vartheta \frac{d^{2}-1}{d^{2}}\right)+\vartheta \frac{d^{2}-1}{d^{2}} \log _{2} \frac{\vartheta}{d^{2}}
$$

holds, while we have

$$
C_{c, 1}(T)=\log _{2}(d)+\left(1-\vartheta \frac{d-1}{d}\right) \log _{2}\left(1-\vartheta \frac{d-1}{d}\right)+\vartheta \frac{d-1}{d} \log _{2} \frac{\vartheta}{d},
$$

where the maximum in Equation (6.25) is achieved for an ensemble of equiprobable pure states taken from an orthonormal basis in $\mathcal{H}$ 82]. This is plausible since the first term under the sup in Equation (6.25) becomes maximal and the second becomes minimal: $\sum_{j} p_{j} T^{*} \rho_{j}$ is maximally mixed in this case and its entropy is therefore maximal. The entropies of the $T^{*} \rho_{j}$ are on the other hand minimal if the $\rho_{j}$ are pure. In Figure 6.2 we have plotted both capacities as a function of the noise parameter $\vartheta$ 
and in Figure 6.3 we have plotted the quotient $C_{e}(T) / C_{c, 1}(T)$ which gives an upper bound on the gain we get from entanglement assistance.

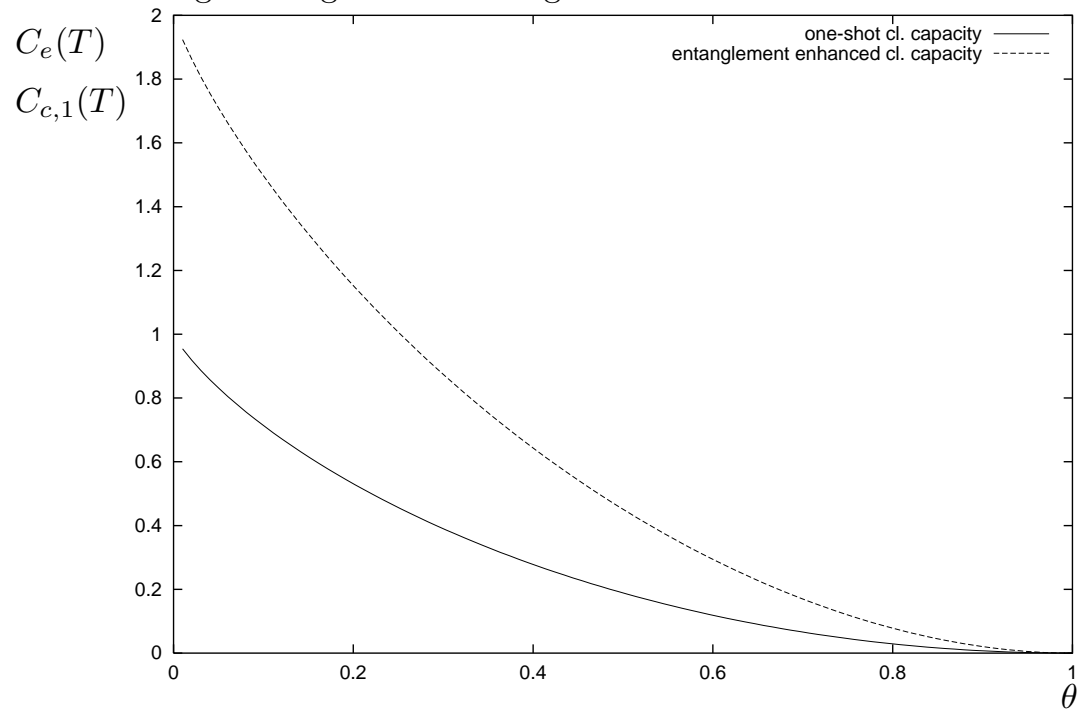

Figure 6.2: Entanglement enhanced and one-shot classical capacity of a depolarizing qubit channel.

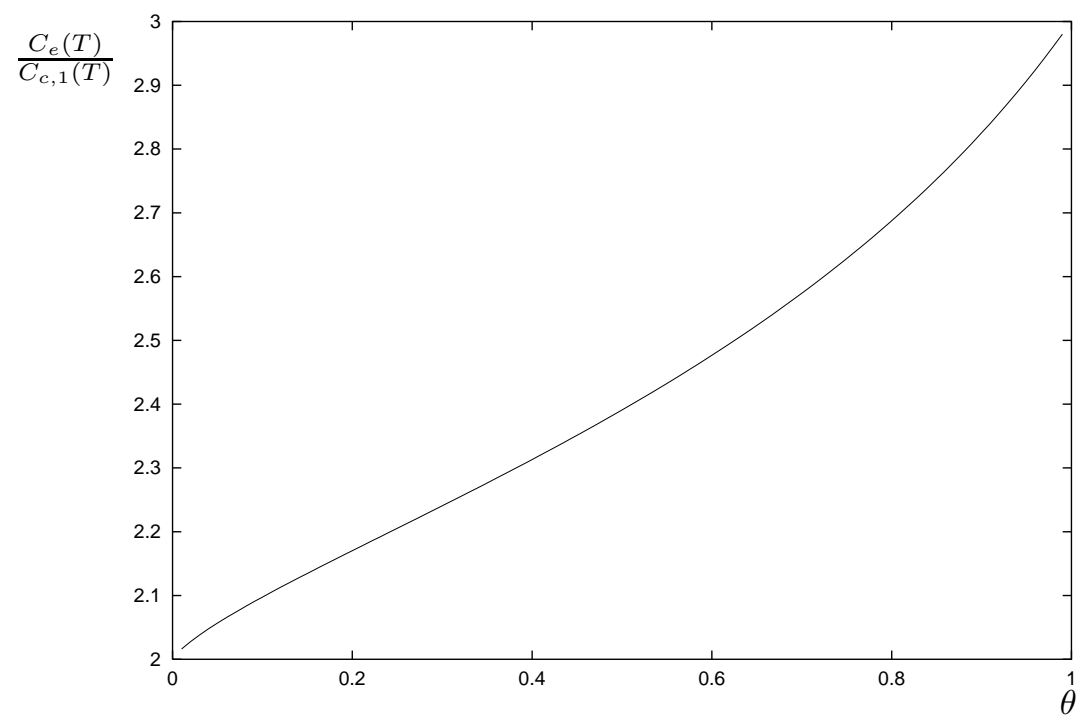

Figure 6.3: Gain of using entanglement assisted versus unassisted classical capacity for a depolarizing qubit channel.

As a third example we want to consider Gaussian channels defined in Subsection 3.3.4. Hence consider the Hilbert space $\mathcal{H}=\mathrm{L}^{2}(\mathbb{R})$ describing a one-dimensional harmonic oscillator (or one mode of the electromagnetic field) and the amplification/attenuation channel $T$ defined in Equation (3.74). The results we want to state concern a slight modification of the original definitions of $C_{c, 1}(T)$ and $C_{e}(T)$ : We will consider capacities for channels with constraint input. This means that only a restricted class of states $\rho$ on the input Hilbert space of the channel are allowed for 
encoding. In our case this means that we will consider the constraint $\operatorname{tr}\left(\rho a a^{*}\right) \leq N$ for a positive real number $N>0$ and with the usual creation and annihilation operators $a^{*}, a$. This can be rewritten as an energy constraint for a quadratic Hamiltonian; hence this is a physically realistic restriction.

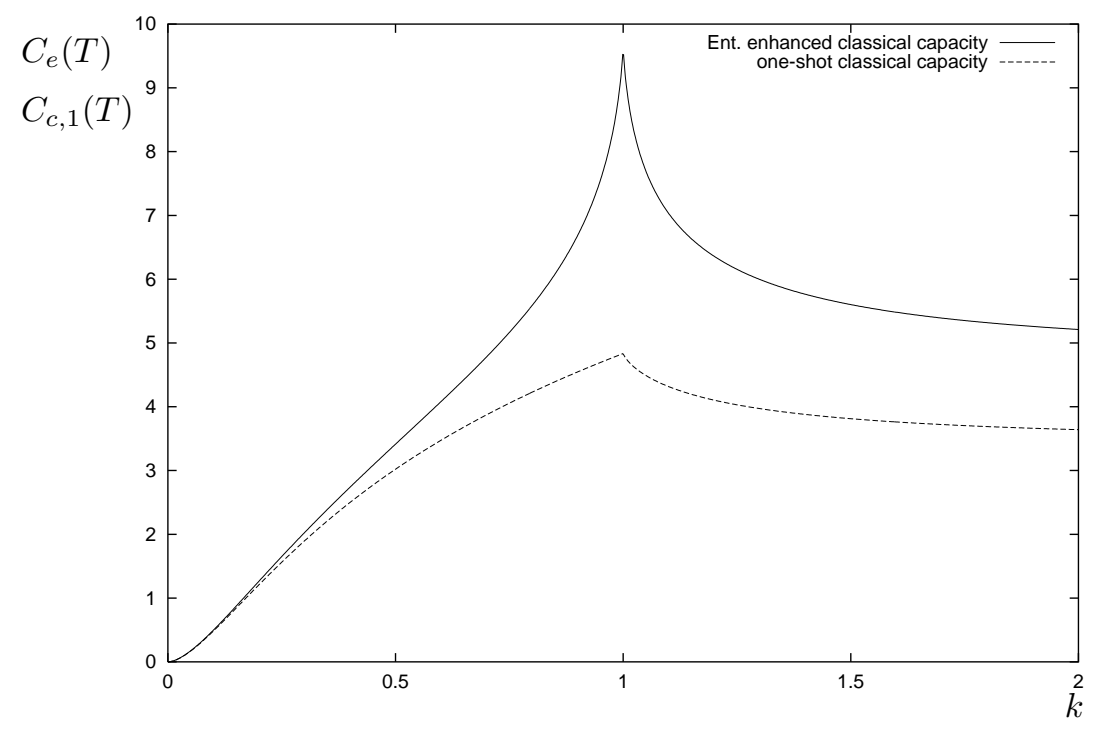

Figure 6.4: One-shot and entanglement enhanced classical capacity of a Gaussian amplification/attenuation channel with $N_{c}=0$ and input noise $N=10$

For the entanglement enhanced capacity it can be shown now that the maximum in Equation (6.28) is taken on Gaussian states. To get $C_{e}(T)$ it is sufficient therefore to calculate the quantum mutual information $I(T, \rho)$ for the Gaussian state $\rho_{N}$ from Equation (3.64). The details can be found in [84 and [18], we will only state the results here. With the abbreviation

$$
g(x)=(x+1) \log _{2}(x+1)-x \log _{2} x
$$

we get $S\left(\rho_{N}\right)=g(N)$ and $S\left(T\left[\rho_{N}\right]\right)=g\left(N^{\prime}\right)$ with $N^{\prime}=k^{2} N+\max \left\{0, k^{2}-1\right\}+N_{c}$ (cf. Equation (3.75)) for the entropies of input and output states and

$$
S(\rho, T)=g\left(\frac{D+N^{\prime}-N-1}{2}\right)+g\left(\frac{D-N^{\prime}+N-1}{2}\right)
$$

with

$$
D=\sqrt{\left(N+N^{\prime}+1\right)^{2}-4 k^{2} N(N+1)}
$$

for the entropy exchange. The sum of all three terms gives $C_{e}(T)$ which we have plotted in Figure 6.4 as a function of $k$.

To calculate the one-shot capacity $C_{c, 1}(T)$ the optimization in Equation 6.25) has to be calculated over probability distributions $p_{j}$ and collections of density operators $\rho_{j}$ such that $\sum_{j} p_{j} \operatorname{tr}\left(a a^{*} \rho_{j}\right) \leq N$ holds. It is conjectured but not yet proven [84. that the maximum is achieved on coherent states with Gaussian probability distribution $p(x)=(\pi N)^{-1} \exp \left(-|x|^{2} / N\right)$. If this is true we get

$$
C_{c, 1}(T)=g\left(N^{\prime}\right)-g\left(N_{0}^{\prime}\right) \text { with } N_{0}^{\prime}=\max \left\{0, k^{2}-1\right\}+N_{c} .
$$


The result is plotted as a function of $k$ in Figure 6.4 and the ratio $G=C_{e} / C_{1}$ in Figure 6.5. $G$ gives an upper bound on the gain of using entanglement assisted versus unassisted classical capacity.

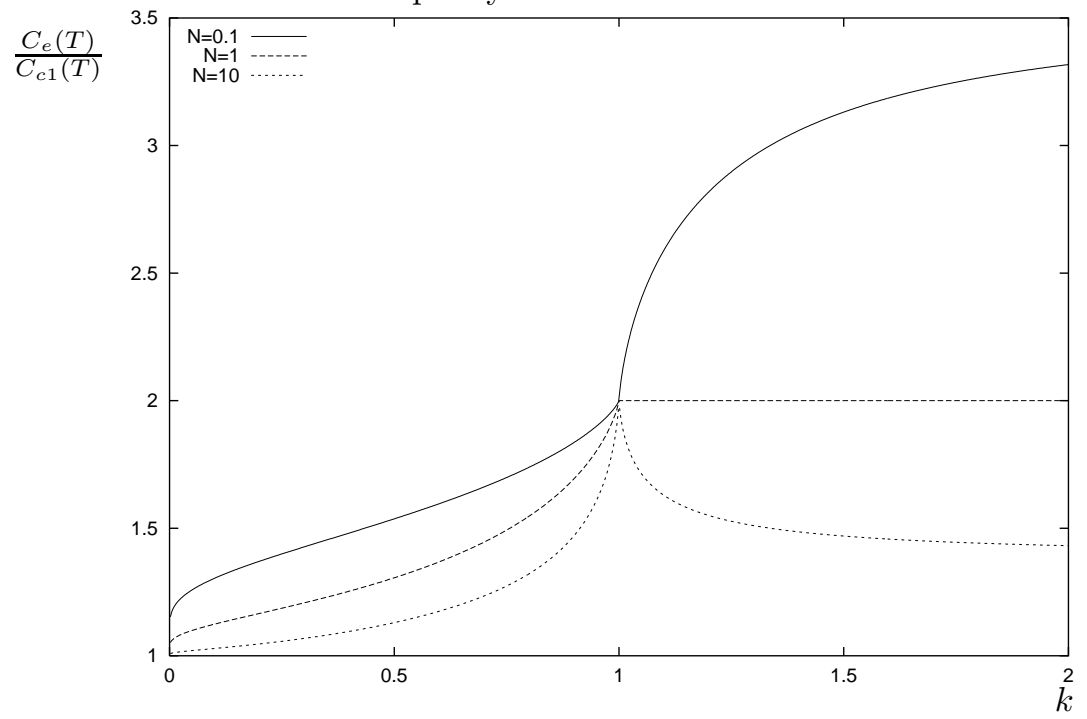

Figure 6.5: Gain of using entanglement assisted versus unassisted classical capacity for a Gaussian amplification/attenuation channel with $N_{c}=0$ and input noise $N=0.1,1,10$

\subsection{The quantum capacity}

The quantum capacity of a quantum channel $T: \mathcal{B}(\mathcal{H}) \rightarrow \mathcal{B}(\mathcal{H})$ is more difficult to treat than the classical capacities discussed in the last section. There is in particular no coding theorem available which would allow explicit calculations. Nevertheless there are partial results available, which we will review in the following.

6.3.1. Alternative definitions. - Let us start with two alternative definitions of $C_{q}(T)$. The first one proposed by Bennett [16] differs only in the error quantity which should go to zero. Instead of the cb-norm the minimal fidelity is used. For a channel $T: \mathcal{B}(\mathcal{H}) \rightarrow \mathcal{B}(\mathcal{H})$ and a subspace $\mathcal{H}^{\prime} \subset \mathcal{H}$ it is defined as

$$
\mathcal{F}_{p}\left(\mathcal{H}^{\prime}, T\right)=\inf _{\psi \in \mathcal{H}^{\prime}}\langle\psi, T[|\psi\rangle\langle\psi|] \psi\rangle
$$

and if $\mathcal{H}^{\prime}=\mathcal{H}$ holds we simply write $\mathcal{F}_{p}(T)$. Hence a number $c$ is an achievable rate if

$$
\lim _{j \rightarrow \infty} \mathcal{F}_{p}\left(E_{j} T^{\otimes M_{j}} D_{j}\right)=1
$$

holds for sequences

$$
E_{j}: \mathcal{B}(\mathcal{H})^{\otimes M_{j}} \rightarrow \mathcal{M}_{2}^{\otimes N_{j}}, \mathcal{D}_{j}: \mathcal{M}_{2}^{\otimes N_{j}} \rightarrow \mathcal{B}(\mathcal{H})^{\otimes M_{j}}, j \in \mathbb{N}
$$

of encodings and decodings and sequences of integers $M_{j}, N_{j}, j \in \mathbb{N}$ satisfying the same constraints as in Definition 6.1.1 (in particular $\lim _{j \rightarrow \infty} N_{j} / M_{j}<c$ ). The equivalence to our version of $C_{q}(T)$ follows now from the estimates 169

$$
\begin{gathered}
\| T-\text { Id }\|\leq\| T-\text { Id } \|_{\mathrm{cb}} \leq 4 \sqrt{\| T-\text { Id } \|} \\
\|T-\operatorname{Id}\| \leq 4 \sqrt{1-\mathcal{F}_{p}(T)} \leq 4 \sqrt{\|T-\operatorname{Id}\| .}
\end{gathered}
$$


A second version of $C_{q}(T)$ is given in [7]. To state it let us define first a quantum source as a sequence $\rho_{N} ; N \in \mathbb{N}$ of density operators $\rho_{N} \in \mathcal{B}^{*}\left(\mathcal{K}^{\otimes N}\right)$ (with an appropriate Hilbert space $\mathcal{K})$ and the entropy rate of this source as $\lim \sup _{N \rightarrow \infty} S\left(\rho_{N}\right) / N$. In addition we need the entanglement fidelity of a state $\rho$ (with respect to a channel $T)$

$$
\mathcal{F}_{e}(\rho, T)=\langle\Psi,(T \otimes \mathrm{Id})[|\Psi\rangle\langle\Psi|] \Psi\rangle,
$$

where $\Psi$ is the purification of $\rho$. Now we define $c \geq 0$ to be achievable if there is a quantum source $\rho_{N}, N \in \mathbb{N}$ with entropy rate $c$ such that

$$
\lim _{n \rightarrow \infty} \mathcal{F}_{e}\left(\rho_{N}, E_{N}^{\prime} T^{\otimes N} D_{N}^{\prime}\right)=1
$$

holds with encodings and decodings

$$
E_{N}^{\prime}: \mathcal{B}(\mathcal{H})^{\otimes N} \rightarrow \mathcal{B}\left(\mathcal{K}^{\otimes N}\right), \mathcal{D}_{N}^{\prime}: \mathcal{B}\left(\mathcal{K}^{\otimes N}\right) \rightarrow \mathcal{B}(\mathcal{H})^{\otimes N}, j \in \mathbb{N} .
$$

Note that these $E_{N}^{\prime}, D_{N}^{\prime}$ play a slightly different role then the $E_{j}, D_{j}$ in Equation (6.39) (and in Definition 6.1.1), because the number of tensor factors of the input and the output algebra is always identical, while in Equation (6.39) the quotients of these numbers lead to the achievable rate. To relate both definitions we have to derive an appropriately chosen family of subspaces $\mathcal{H}_{N}^{\prime} \subset \mathcal{K}^{\otimes N}$ from the $\rho_{N}$ such that the minimal fidelities $\mathcal{F}_{p}\left(\mathcal{H}_{N}^{\prime}, E_{N}^{\prime} T^{\otimes N} D_{N}^{\prime}\right)$ of these subspaces go to 1 as $N \rightarrow \infty$. If we identify the $\mathcal{H}_{N}^{\prime}$ with tensor products of $\mathbb{C}^{2}$ and the $E_{j}, D_{j}$ of Equation (6.39) with restrictions of $E_{N}^{\prime}, D_{N}^{\prime}$ to these tensor products we recover Equation (6.38). A precise implementation of this rough idea can be found in [6] and it shows that both definitions just discussed are indeed equivalent.

6.3.2. Upper bounds and achievable rates. - Although there is no coding theorem for the quantum capacity $C_{q}(T)$, there is a fairly good candidate which is related to the coherent information

$$
J(\rho, T)=S\left(T^{*} \rho\right)-S(\rho, T) .
$$

Here $S\left(T^{*} \rho\right)$ is the entropy of the output state and $S(\rho, T)$ is the entropy exchange defined in Equation (6.26). It is argued [7] that $J(\rho, T)$ plays a role in quantum information theory which is analogous to that of the (classical) mutual information (6.21) in classical information theory. $J(\rho, T)$ has some nasty properties, however: it can be negative [41] and it is known to be not additive [54]. To relate it to $C_{q}(T)$ it is therefore not sufficient to consider a one-shot capacity as in Shannons Theorem (Thm 6.2.1). Instead we have to define

$$
C_{s}(T)=\sup _{N} \frac{1}{N} C_{s, 1}\left(T^{\otimes N}\right) \text { with } C_{s, 1}(T)=\sup _{\rho} J(\rho, T) .
$$

In [7] and [8] it is shown that $C_{s}(T)$ is an upper bound on $C_{q}(T)$. Equality, however, is conjectured but not yet proven, although there are good heuristic arguments [110], 90].

A second interesting quantity which provides an upper bound on the quantum capacity uses the transposition operation $\Theta$ on the output systems. More precisely it is shown in 84 that

$$
C_{q}(T) \leq C_{\theta}(T)=\log _{2}\|T \Theta\|_{\mathrm{cb}}
$$

holds for any channel. In contrast to many other calculations in this field it is particular easy to derive this relation from properties of the cb-norm. Hence we are able to give a proof here. We start with the fact that $\|\Theta\|_{\mathrm{cb}}=d$ if $d$ is the dimension 
of the Hilbert space on which $\Theta$ operates. Assume that $N_{j} / M_{j} \rightarrow c \leq C_{q}(T)$ and $j$ large enough such that $\left\|\operatorname{Id}_{2}^{N_{j}}-E_{j} T^{\otimes M_{j}} D_{j}\right\| \leq \epsilon$ with appropriate encodings and decodings $E_{j}, D_{j}$. We get

$$
\begin{aligned}
2^{N_{j}} & =\left\|\operatorname{Id}_{2}^{N_{j}} \Theta\right\|_{\mathrm{cb}} \leq\left\|\Theta\left(\operatorname{Id}_{2}^{N_{j}}-E_{j} T^{\otimes M_{j}} D_{j}\right)\right\|_{\mathrm{cb}}+\left\|\Theta E_{j} T^{\otimes M_{j}} D_{j}\right\|_{\mathrm{cb}} \\
& \leq 2^{N_{j}}\left\|\operatorname{Id}_{2}^{N_{j}}-E_{j} T^{\otimes M_{j}} D_{j}\right\|_{\mathrm{cb}}+\left\|\Theta E_{j} \Theta(\Theta T)^{\otimes M_{j}} D_{j}\right\|_{\mathrm{cb}} \\
& \leq 2^{N_{j}} \epsilon+\|\Theta T\|_{\mathrm{cb}}^{M_{j}}
\end{aligned}
$$

where we have used for the last equation the fact that $D_{j}$ and $\Theta E_{j} \Theta$ are channels and that the cb-norm is multiplicative. Taking on both sides the logarithm we get

$$
\frac{N_{j}}{M_{j}}+\frac{\log _{2}(1-\epsilon)}{M_{j}} \leq \log _{2}\|\Theta T\|_{\mathrm{cb}} .
$$

In the limit $j \rightarrow \infty$ this implies $c \leq \log _{2}\|\Theta T\|$ and therefore $C_{q}(T) \leq \log _{2}\|\Theta T\|_{\mathrm{cb}}=$ $C_{\theta}(T)$ as stated.

Since $C_{\theta}(T)$ is an upper bound on $C_{q}(T)$ it is particularly useful to check whether the quantum capacity for a particular channel is zero. If, e.g. $T$ is classical we have $\Theta T=T$ since the transposition coincides on a classical algebra $\mathcal{C}_{d}$ with the identity (elements of $\mathcal{C}_{d}$ are just diagonal matrices). This implies $C_{\theta}(T)=\log _{2}\|\Theta T\|_{\mathrm{cb}}=$ $\log _{2}\|T\|_{\mathrm{cb}}=0$, because the cb-norm of a channel is 1 . We see therefore that the quantum capacity of a classical channel is 0 - this is just another proof of the no-teleportation theorem. A slightly more general result concerns channels $T=$ $R S$ which are the composition of a preparation $R: \mathcal{M}_{d} \rightarrow \mathcal{C}_{f}$ and a subsequent measurement $S: \mathcal{C}_{f} \rightarrow \mathcal{M}_{d}$. It is easy to see that $\Theta T=\Theta R S$ is a channel, because $\Theta R \Theta$ is a channel and $\Theta$ is the identity on $\mathcal{C}_{f}$, hence $\Theta R \Theta=\Theta R$ and $\Theta R \Theta S=$ $\Theta R S=\Theta T$. Again we get $C_{\theta}(T)=0$.

Let us consider now some examples. The most simple case is again the quantum erasure channel from Equation (6.29). As for the classical capacities its quantum capacity can be explicitly calculated 15] and we have $C_{q}(T)=\max \left(0,(1-2 \vartheta) \log _{2}(d)\right)$; cf. Figure 6.1.

For the depolarizing channel (6.30) precise calculations of $C_{q}(T)$ are not availailable. Hence let us consider first the coherent information. $J(T, \rho)$ inherits from $T$ its unitary covariance, i.e. we have $J\left(U \rho U^{*}, T\right)=J(\rho, T)$. In contrast to the mutual information, however, it does not have nice cocavity properties, which makes the optimization over all input states more difficult to solve. Nevertheless, the calculation of $J(\rho, T)$ is straightforward and we get in the qubit case (if $\vartheta$ is the noise parameter of $T$ and $\lambda$ is the highest eigenvalue of $\rho$ ):

$$
\begin{array}{r}
J(\rho, T)=S\left(\lambda(1-\vartheta)+\frac{\vartheta}{2}\right)-S\left(\frac{1-\vartheta / 2+A}{2}\right)-S\left(\frac{1-\vartheta / 2-A}{2}\right) \\
-S\left(\frac{\lambda \vartheta}{2}\right)-S\left(\frac{(1-\lambda) \vartheta}{2}\right)
\end{array}
$$

where $S(x)=-x \log _{2}(x)$ denotes again the entropy function and

$$
A=\sqrt{(2 \lambda-1)^{2}(1-\vartheta / 2)^{2}+4 \lambda(1-\lambda)(1-\vartheta)^{2}} .
$$

Optimization over $\lambda$ can be performed at least numerically (the maximum is attained at the left boundary $(\lambda=1 / 2)$ if $J$ is positive there, and the right boundary otherwise). The result is plotted together with $C_{\theta}(T)$ in Figure 6.6 as a function of $\theta$. The quantity $C_{\theta}(T)$ is much easier to compute and we get

$$
C_{\theta}(T)=\max \left\{0, \log _{2}\left(2-\frac{3}{2} \theta\right)\right\} .
$$


To get a lower bound on $C_{q}(T)$ we have to show that a certain rate $r \leq C_{q}(T)$ can be achieved with an appropriate sequence

$$
E_{M}: \mathcal{M}_{d}^{\otimes M} \rightarrow \mathcal{M}_{2}^{\otimes N(M)}, \quad M, N(M) \in \mathbb{N}
$$

of error correcting codes and corresponding decodings $D_{M}$. I.e. we need

$$
\lim _{j \rightarrow \infty} N(M) / M=r \text { and } \lim _{j \rightarrow \infty}\left\|E_{M} T^{\otimes M} D_{M}-\mathrm{Id}\right\|_{\mathrm{cb}}=0
$$

To find such a sequence note first that we can look at the depolarizing channel as a device which produces an error with probability $\vartheta$ and leaves the quantum information intact otherwise. If more and more copies of $T$ are used in parallel, i.e. if $M$ goes to infinity, the number of errors approaches therefore $\vartheta M$. In other words the probability to have more than $\vartheta M$ errors vanishes asymptotically. To see this consider

$$
T^{\otimes M}=\left((\vartheta-1) \operatorname{Id}+\vartheta d^{-1} \operatorname{tr}(\cdot) \mathbb{I}\right)^{\otimes M}=\sum_{K=1}^{M}(1-\vartheta)^{K} \vartheta^{N-K} T_{K}^{(M)}
$$

where $T_{K}^{(M)}$ denotes the sum of all $M$-fold tensor products with $d^{-1} \operatorname{tr}(\cdot) \mathbb{I}$ on $N$ places and Id on the $N-K$ remaining - i.e. $T_{K}^{(M)}$ is a channel which produces

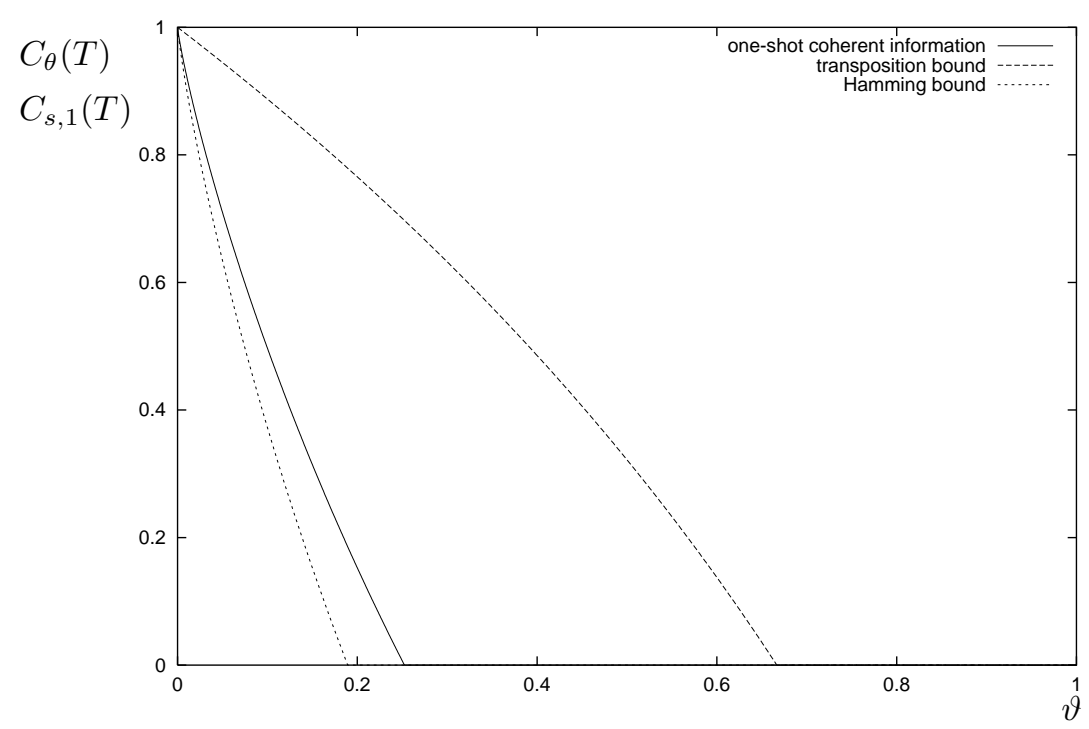

Figure 6.6: $C_{\theta}(T), C_{s}(T)$ and the Hamming bound of a depolarizing qubit channel plotted as function of the noise parameter $\vartheta$. 
exactly $K$ errors on $M$ transmitted systems. Now we have

$$
\begin{aligned}
\| T^{\otimes M}-\sum_{K \leq \vartheta M}(1-\vartheta)^{K} & \vartheta^{N-K} T_{K}^{(M)} \|_{\mathrm{cb}} \\
& =\left\|\sum_{K>\vartheta M}(1-\vartheta)^{K} \vartheta^{N-K} T_{K}^{(M)}\right\|_{\mathrm{cb}} \\
& \leq \sum_{K>\vartheta M}^{M}(1-\vartheta)^{K} \vartheta^{N-K}\left\|T_{K}^{(M)}\right\|_{\mathrm{cb}} \\
& \leq \sum_{K>\vartheta M}^{M}\left(\begin{array}{c}
M \\
K
\end{array}\right)(1-\vartheta)^{K} \vartheta^{N-K}=R .
\end{aligned}
$$

The quantity $R$ is the tail a of Binomial series and vanishes therefore in the limit $M \rightarrow \infty$ (cf. e.g. Appendix B of 131). This shows that for $M \rightarrow \infty$ only terms $T_{K}^{(M)}$ with $K \leq \vartheta M$ are relevant in Equation $(6.57)$ - in other words at most $\vartheta M$ errors occur asymptotically, as stated. This implies that we need a sequence of codes $E_{M}$ which encode $N(M)$ qubits and correct $\vartheta M$ errors on $M$ places. One way to get such a sequence is "random coding" - the classical version of this method is well known from the proof of Shannons theorem. The idea is, basically, to generate error correcting codes of a certain type randomly. E.g. we can generate a sequence of random graphs with $N(M)$ input and $M$ output vertices (cf. Section 4.4). If we can show that the corresponding codes correct (asymptotically) $\vartheta M$ errors, the corresponding rate $r=\lim _{M \rightarrow \infty} N(M) / M$ is achievable. For the depolarizing channel2 such an analysis, using randomly generated stabilizer codes shows [16, 71]

$$
C_{q}(T) \leq 1-H(\vartheta)-\vartheta \log _{2} 3
$$

where $H$ is the binary entropy from Equation (5.16). This bound can be further improved using a more clever coding strategy; cf. [54.

As a third example let us consider again the Gaussian channel studied already in Subsection 6.2.4. For $C_{\theta}(T)$ we have (the corresponding calculation is not trivial and uses properties of Gaussian channels which we have not discussed; cf. [84].)

$$
C_{\theta}(T)=\max \left\{0, \log _{2}\left(k^{2}+1\right)-\log _{2}\left(\left|k^{2}-1\right|+2 N_{c}\right)\right\},
$$

and we see that $C_{\theta}(T)$ and therefore $C_{q}(T)$ become zero if $N_{c}$ is large enough (i.e. $N_{c} \geq \max \left\{1, k^{2}\right\}$ ). The coherent information for the Gaussian state $\rho_{N}$ from Equation (3.64) has the form

$$
J\left(\rho_{N}, T\right)=g\left(N^{\prime}\right)-g\left(\frac{D+N^{\prime}-N-1}{2}\right)-g\left(\frac{D-N^{\prime}+N-1}{2}\right)
$$

with $N^{\prime}, D$ and $g$ as in Subsection 6.2.4. It increases with $N$ and we can calculate therefore the maximum over all Gaussian states (which might differ from $C_{S}(T)$ ) as

$$
C_{G}(T)=\lim _{N \rightarrow \infty} J\left(\rho_{N}, T\right)=\log _{2} k^{2}-\log _{2}\left|k^{2}-1\right|-g\left(\frac{N_{c}}{k^{2}-1}\right) .
$$

We have plotted both quantities in Figure 6.7 as a function of $k$.

Finally let us have a short look on the special case $k=1$, i.e. $T$ describes in this case only the influence of classical Gaussian noise on the transmitted qubits. If we set

\footnotetext{
${ }^{2}$ With a more thorough discussion similar results can be obtained for a.much more general class of channels, e.g. all $T$ in a neighbourhood of the identity channel; cf. 114.
} 


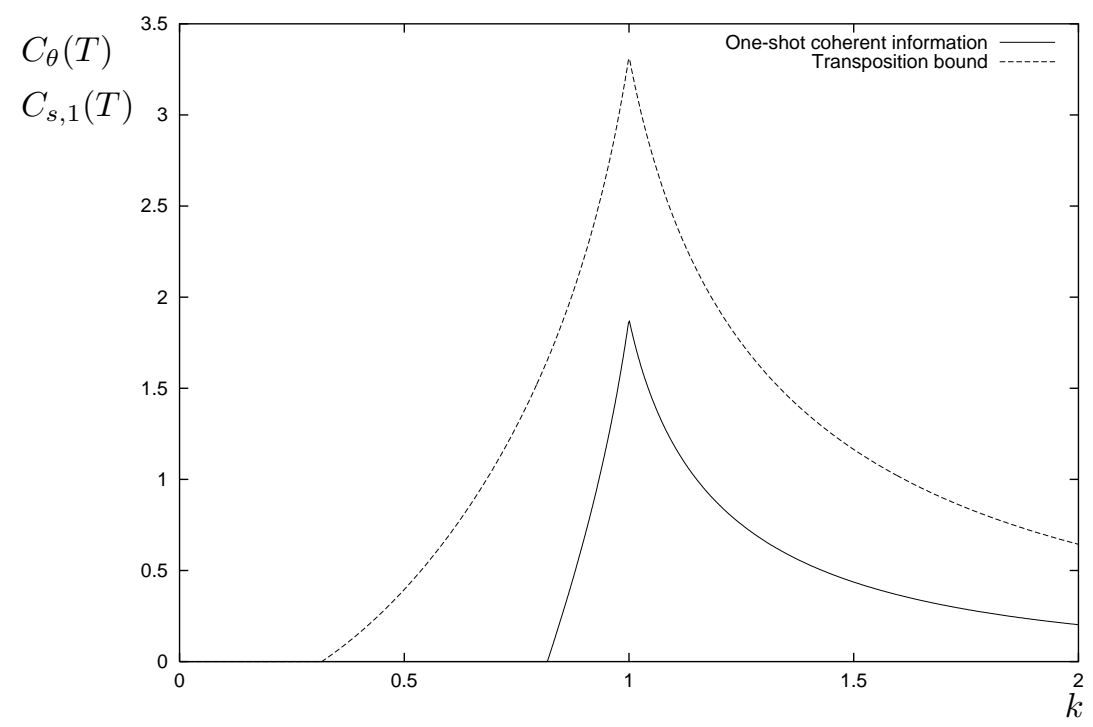

Figure 6.7: $C_{\theta}(T)$ and $C_{s}(T)$ of a Gaussian amplification/attenuation channel as a function of amplification parameter $k$.

$k=1$ in Equation (6.64) and take the limit $N \rightarrow \infty$ we get $C_{G}(T)=-\log _{2}\left(N_{c} e\right)$ and $C_{\theta}(T)$ becomes $C_{\theta}(T)=\max \left\{0,-\log _{2}\left(N_{c}\right)\right\}$; both quantities are plotted in Figure 6.8. This special case is interesting because the one-shot coherent information $C_{G}(T)$ is achievable, provided the noise parameter $N_{c}$ satisfies certain conditions [77. Hence there is strong evidence that the quantum capacity lies between the two lines in Figure 6.8.

6.3.3. Relations to entanglement measures. - The duality lemma proved in Subsection 2.3.3 provides an interesting way to derive bounds on channel capacities and capacity like quantities from entanglement measures (and vice versa) [16, 90: To derive a state of a bipartite system from a channel $T$ we can take a maximally entangled state $\Psi \in \mathcal{H} \otimes \mathcal{H}$, send one particle through $T$ and get a less entangled pair in the state $\rho_{T}=\left(\operatorname{Id} \otimes T^{*}\right)|\Psi\rangle\langle\Psi|$. If on the other hand an entangled state $\rho \in \mathcal{S}(\mathcal{H} \otimes \mathcal{H})$ is given, we can use it as a recource for teleportation and get a channel $T_{\rho}$. The two maps $\rho \mapsto T_{\rho}$ and $T \mapsto \rho_{T}$ are, however, not inverse to one another. This can be seen easily from the duality lemma (Theorem 2.3.4): For each state $\rho \in \mathcal{S}(\mathcal{H} \otimes \mathcal{H})$ there is a channel $T$ and a pure state $\Phi \in \mathcal{H} \otimes \mathcal{H}$ such that $\rho=\left(\operatorname{Id} \otimes T^{*}\right)|\Phi\rangle\langle\Phi|$ holds; but $\Phi$ is in general not maximally entangled (and uniquely determined by $\rho$ ). Nevertheless, there are special cases in which the state derived from $T_{\rho}$ coincides with $\rho$ : A particular class of examples is given by teleportation channels derived from a Bell-diagonal state.

On $\rho_{T}$ we can evaluate an entanglement measure $E\left(\rho_{T}\right)$ and get in this way a quantity which is related to the capacity of $T$. A particularly interesting candidate for $E$ is the "one-way LOCC" distillation rate $E_{D, \rightarrow}$. It is defined in the same way as the entanglement of distillation $E_{D}$, except that only one-way LOCC operation are allowed in Equation (5.8). According to [16] $E_{D, \rightarrow}$ is related to $C_{q}$ by the inequalities $E_{D, \rightarrow}(\rho) \geq C_{q}\left(T_{\rho}\right)$ and $E_{D, \rightarrow}\left(T_{\rho}\right) \leq C_{q}(T)$. Hence if $\rho_{T_{\rho}}=\rho$ we can calculate $E_{D, \rightarrow}(\rho)$ in terms of $C_{q}\left(T_{\rho}\right)$ and vice versa.

\footnotetext{
${ }^{3}$ It is only shown that $\log _{2}\left(\left\lfloor 1 /\left(N_{c} e\right)\right\rfloor\right)$ can be achieved, where $\lfloor x\rfloor$ denotes the biggest integer less than $x$. It is very likely however that this is only a restriction of the methods used in the proof and not of the result.
} 


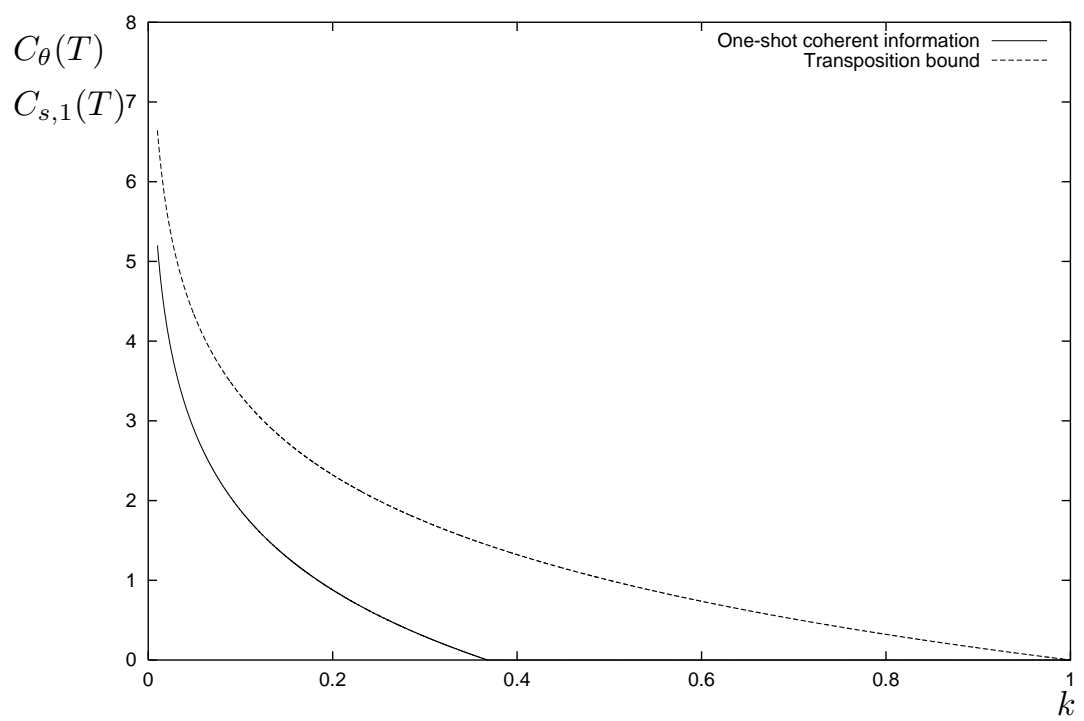

Figure 6.8: $C_{\theta}(T)$ and $C_{s}(T)$ of a Gaussian amplification/attenuation channel as a function of the noise parameter $N_{c}$ (and with $k=1$ ).

A second interesting example is the transposition bound $C_{\theta}(T)$ introduced in the last subsection. It is related to the logarithmic negativity 158

$$
E_{\theta}\left(\rho_{T}\right)=\log _{2}\left\|(\operatorname{Id} \otimes \Theta) \rho_{T}\right\|_{1}
$$

which measures the degree with which the partial transpose of $\rho$ fails to be positive. $E_{\theta}$ can be regarded as entanglement measure although it has some drawbacks: it is not LOCC monotone (Axiom E2), it is not convex (Axiom E3) and most severe: It does not coincides with the reduced von Neumann entropy on pure states, which we have considered as "the" entanglement measure for pure states. On the other hand it is easy to calculate and it gives bounds on distillation rates and teleportation capacities 158. In addition $E_{\theta}$ can be used together with the relation between depolarizing channels and isotropic states to derive Equation (6.54) in a very simple way. 


\section{Chapter 7}

\section{Multiple inputs}

We have seen in Chapter 1 that many tasks of quantum information which are impossible with one-shot operations can be approximated by channels which operate on a large number of equally prepared inputs. Typical examples are approximate cloning, undoing noise and distillation of entanglement. There are basically two questions which are interesting for a quantitative analysis: First we can search for the optimal solutions for a fixed number $N$ of input systems and second we can ask for the asymptotic behavior in the limit $N \rightarrow \infty$. In the latter case the asymptotic rate, i.e. the number of outputs (of a certain quality) per input system is of particular interest.

\subsection{The general scheme}

Both types of questions just mentioned can be treated (up to certain degree) independently from the (impossible) task we are dealing with and we will study in the following the corresponding general scheme. Hence consider a channel $T: \mathcal{B}\left(\mathcal{H}^{\otimes M}\right) \rightarrow \mathcal{B}\left(\mathcal{H}^{\otimes N}\right)$ which operates on $N$ input systems and produces $M$ outputs of the same type. Our aim is to optimize a "figure of merit" $\mathcal{F}(T)$ which measures the deviation of $T^{*}\left(\rho^{\otimes N}\right)$ from the target functional we want to approximate. The particular type of device we are considering is mainly fixed by the choice of $\mathcal{F}(T)$ and we will discuss in the following the most relevant examples. (Note that we have considered them already on a qualitative level in Chapter 4 ; cf. in particular Section 4.2 and 4.3 ).

7.1.1. Figures of merit. - Let us start with pure state cloning 68, 31, 32, 35, 167, 98], i.e. for each (unknown) pure input state $\sigma=|\psi\rangle\langle\psi|, \psi \in \mathcal{H}$ the $M$ clones $T^{*}\left(\sigma^{\otimes N}\right)$ produced by the channel $T$ should approximate $M$ copies of the input in the common state $\sigma^{\otimes M}$ as good as possible. There are in fact two different possibilities to measure the distance of $T^{*}\left(\sigma^{\otimes N}\right)$ to $\sigma^{\otimes M}$. We can either check the quality of each clone separately or we can test in addition the correlations between output systems. With the notation

$$
\sigma^{(j)}=\mathbb{I}^{\otimes(j-1)} \otimes \sigma \otimes \mathbb{I}^{\otimes(M-j)} \in \mathcal{B}\left(\mathcal{H}^{\otimes M}\right)
$$

a figure of merit for the first case is given by

$$
\mathcal{F}_{c, 1}(T)=\inf _{j=1, \ldots, N} \inf _{\sigma \text { pure }} \operatorname{tr}\left(\sigma^{(j)} T^{*}\left(\sigma^{\otimes N}\right)\right) .
$$

It measures the worst one particle fidelity of the output state $T^{*}\left(\sigma^{\otimes N}\right)$. If we are interested in correlations too, we have to choose

$$
\mathcal{F}_{c, \text { all }}(T)=\inf _{\sigma \text { pure }} \operatorname{tr}\left(\sigma^{\otimes M} T^{*}\left(\sigma^{\otimes N}\right)\right)
$$

which is again a "worst case" fidelity, but now of the full output with respect to $M$ uncorrelated copies of the input $\sigma$.

Instead of fidelities we can consider other error quantities like trace-norm distances or relative entropies. In general, however, we do not get significantly different results from such alternative choices; hence we can safely ignore them. Real variants arise if we consider instead of the infima over all pure states quantities which prefer a (possibly discrete or even finite) class of states. Such a choice leads to "state dependent cloning", because the corresponding optimal devices perform better as "universal" ones (i.e. those described by the figures of merit above) on some 
states but much worse on the rest. We ignore state dependent cloning in this work, because the universal case is physically more relevant and technically more challenging. Other cases which we do not discuss either include "asymmetric cloning", which arises if we trade in Equation (7.2) the quality of one particular output system against the rest (see [40]), and cloning of mixed states. The latter is much more difficult then the pure state case and even for classical systems, where it is related to the so called "bootstrap" technique [59], nontrivial.

Closely related to cloning is purification, i.e. undoing noise. This means we are considering $N$ systems originally prepared in the same (unknown) pure state $\sigma$ but which have passed a depolarizing channel

$$
R^{*} \sigma=\vartheta \sigma+(1-\vartheta) \mathbb{I} / d
$$

afterwards. The task is now to find a device $T$ acting on $N$ of the decohered systems such that $T^{*}\left(R^{*} \sigma\right)$ is as close as possible to the original pure state. We have the same basic choices for a figure of merit as in the cloning problem. Hence we define

$$
\mathcal{F}_{R, 1}(T)=\inf _{j=1, \ldots, N} \inf _{\sigma \text { pure }} \operatorname{tr}\left(\sigma^{(j)} T^{*}\left[\left(R^{*} \sigma\right)^{\otimes N}\right]\right)
$$

and

$$
\mathcal{F}_{R, \text { all }}(T)=\inf _{\sigma \text { pure }} \operatorname{tr}\left(\sigma^{\otimes M} T^{*}\left[\left(R^{*} \sigma\right)^{\otimes N}\right]\right) .
$$

These quantities can be regarded as generalizations of $\mathcal{F}_{c, 1}$ and $\mathcal{F}_{c \text {,all }}$ which we recover if $R^{*}$ is the identity.

Another task we can consider is the approximation of a map $\Theta$ which is positive but not completely positive, like the transposition. Positivity and normalization imply that $\Theta^{*}$ maps states to states but $\Theta$ can not be realized by a physical device. An explicit example is the universal not gate (UNOT) which maps each pure qubit state $\sigma$ to its orthocomplement $\sigma^{\perp}[36$. It is given the the anti-unitary operator

$$
\psi=\alpha|0\rangle+\beta|1\rangle \mapsto \Theta \psi=\bar{\alpha}|0\rangle-\bar{\beta}|1\rangle .
$$

Since $\Theta \sigma$ is a state if $\sigma$ is, we can ask again for a channel $T$ such that $T^{*}\left(\sigma^{\otimes N}\right)$ approximates $(\Theta \sigma)^{\otimes M}$. As in the two previous examples we have the choice to allow arbitrary correlations in the output or not and we get the following figures of merit:

$$
\mathcal{F}_{\theta, 1}(T)=\inf _{j=1, \ldots, N} \inf _{\sigma \text { pure }} \operatorname{tr}\left((\Theta \sigma)^{(j)} T^{*}\left(\sigma^{\otimes N}\right)\right)
$$

and

$$
\mathcal{F}_{\theta, \text { all }}(T)=\inf _{\sigma \text { pure }} \operatorname{tr}\left((\Theta \sigma)^{\otimes M} T^{*}\left(\sigma^{\otimes N}\right)\right) .
$$

Note that we can plug in for $\Theta$ basically any functional which maps states to states. In addition we can combine Equation (7.5) and (7.6) on the one hand with (7.8) and 7.9 ) on the other. As result we would get a measure for devices which undo an operation $R$ and approximate an impossible machine $\Theta$ at the same time.

7.1.2. Covariant operations. - All the functionals just defined give rise to optimization problems which we will study in greater detail in the next Sections. This means we are interested in two things: First of all the maximal value of $\mathcal{F}_{\#, \emptyset}$ (with $\#=c, R, \theta$ and $\emptyset=1$, all) given by

$$
\mathcal{F}_{\#, \natural}(N, M)=\inf _{T} \mathcal{F}_{\#, \natural}(T),
$$


where the supremum is taken over all channels $T: \mathcal{B}\left(\mathcal{H}^{\otimes M}\right) \rightarrow \mathcal{B}\left(\mathcal{H}^{\otimes N}\right)$, and second the particular channel $\widehat{T}$ where the optimum is attained. At a first look a complete solution of these problems seems to be impossible, due to the large dimension of the space of all $T$, which scales exponentially in $M$ and $N$. Fortunately all $\mathcal{F}_{\#, \sharp}(T)$ admit a large symmetry group which allows in many cases the explicit calculation of the optimal values $\mathcal{F}_{\#, \mathfrak{t}}(N, M)$ and the determination of optimizers $\widehat{T}$ with a certain covariance behavior. Note that this is an immediate consequence of our decision to restrict the discussion to "universal" procedures, which do not prefer any particular input state.

Let us consider permutations of the input systems first: If $p \in \mathrm{S}_{N}$ is a permutation on $N$ places and $V_{p}$ the corresponding unitary on $\mathcal{H}^{\otimes N}$ (cf. Equation (3.7)) we get obviously $T^{*}\left(V_{p} \rho^{\otimes N} V_{p}^{*}\right)=T^{*}\left(\rho^{\otimes N}\right)$, hence

$$
\mathcal{F}_{\#, \sharp}\left[\alpha_{p}(T)\right]=\mathcal{F}_{\#, \natural}(T) \forall p \in \mathrm{S}_{N} \text { with }\left[\alpha_{p}(T)\right](A)=V_{p}^{*} T(A) V_{p} .
$$

In other words: $\mathcal{F}_{\#, \natural}(T)$ is invariant under permutations of the input systems. Similarly we can show that $\mathcal{F}_{\#, \mathfrak{\natural}}(T)$ is invariant under permutations of the output systems:

$$
\mathcal{F}_{\#, \natural}\left[\beta_{p}(T)\right]=\mathcal{F}(T) \forall p \in \mathrm{S}_{M} \text { with }\left[\beta_{p}(T)\right](A)=T\left(V_{p}^{*} A V_{p}\right) .
$$

To see this consider e.g. for $\#=c$ and $\downarrow=$ all

$$
\operatorname{tr}\left[\sigma^{\otimes M} V_{p} T^{*}\left(\rho^{\otimes N}\right) V_{p}^{*}\right]=\operatorname{tr}\left[V_{p} \sigma^{\otimes M} V_{p}^{*} T^{*}\left(\rho^{\otimes N}\right)\right]=\operatorname{tr}\left[\sigma^{\otimes M} T^{*}\left(\rho^{\otimes N}\right)\right] .
$$

For the other cases similar calculations apply.

Finally, none of the $\mathcal{F}_{\#, \natural}(T)$ singles out a preferred direction in the one particle Hilbert space $\mathcal{H}$. This implies that we can rotate $T$ by local unitaries of the form $U^{\otimes N}$ respectively $U^{\otimes M}$ without changing $\mathcal{F}_{\#, \mathfrak{\natural}}(T)$. More precisely we have

$$
\mathcal{F}_{\#, \mathfrak{\natural}}\left[\gamma_{U}(T)\right]=\mathcal{F}_{\#, \mathfrak{\natural}}(T) \forall U \in \mathrm{U}(d)
$$

with

$$
\left[\gamma_{U}(T)\right](A)=U^{* \otimes N} T\left(U^{\otimes M} A U^{* \otimes M}\right) U^{\otimes N} .
$$

The validity of Equation (7.14) can be proven in the same way as (7.11) and (7.12). The details are therefore left to the reader.

Now we can average over the groups $S_{N}, S_{M}$ and $\mathrm{U}(d)$. Instead of the operation $T$ we consider

$$
\bar{T}=\frac{1}{N ! M !} \sum_{p \in S_{N}} \sum_{q \in S_{M}} \int_{G} \alpha_{p} \beta_{q} \gamma_{U}(T) d U
$$

where $d U$ denotes the normalized, left invariant Haar measure on $\mathrm{U}(d)$. We see immediately that $\bar{T}$ has the following symmetry properties

$$
\alpha_{p}(\bar{T})=\bar{T}, \beta_{q}(\bar{T})=\bar{T}, \gamma_{U}(\bar{T})=\bar{T}, \forall p \in S_{N}, \forall q \in S_{M}, \forall U \in \mathrm{U}(d)
$$

and we will call each operation $T$ fully symmetric, if it satisfies this equation. The concavity of $\mathcal{F}_{\#, \downarrow}$ implies immediately that it can not decrease if we replace $T$ by $\bar{T}$ :

$$
\begin{aligned}
\mathcal{F}_{\#, \natural}(T) & =\mathcal{F}_{\#, \natural}\left(\frac{1}{N ! M !} \sum_{p \in S_{N}} \sum_{q \in S_{M}} \int_{G} \alpha_{p} \beta_{q} \gamma_{U}(T) d U\right) \\
& \geq \frac{1}{N ! M !} \sum_{p \in S_{N}} \sum_{q \in S_{M}} \int_{G} \mathcal{F}_{\#, \text { घ }}\left[\alpha_{p} \beta_{q} \gamma_{U}(T)\right] d U=\mathcal{F}_{\#, \natural}(T) .
\end{aligned}
$$


To calculate the optimal value $\mathcal{F}_{\#, \downarrow}(N, M)$ it is therefore completely sufficient to search a maximizer for $\mathcal{F}_{\#, \downarrow}(T)$ only among fully symmetric $T$ and to evaluate $\mathcal{F}_{\#, \mathfrak{t}}(T)$ for this particular operation. This simplifies the problem significantly because the size of the parameter space is extremely reduced. Of course we do not know from this argument whether the optimum is attained on non-symmetric operations, however this information is in general less important (and for some problems like optimal cloning a uniqueness result is available).

7.1.3. Group representations. - To get an idea how this parameter reduction can be exploited practically, let us reconsider Theorem 3.1.1: The two representations $U \mapsto U^{\otimes N}$ and $p \mapsto V_{p}$ of $\mathrm{U}(d)$ respectively $\mathrm{S}_{N}$ on $\mathcal{H}^{\otimes N}$ are "commutants" of each other, i.e., any operator on $\mathcal{H}^{\otimes N}$ commuting with all $U^{\otimes N}$ is a linear combination of the $V_{p}$, and conversely. This knowledge can be used to decompose the representation $U^{\otimes N}$ (and $V_{p}$ as well) into irreducible components. To reduce the group theoretic overhead, we will discuss this procedure first for qubits only and come back to the general case afterwards.

Hence assume that $\mathcal{H}=\mathbb{C}^{2}$ holds. Then $\mathcal{H}^{\otimes N}$ is the Hilbert space of $N$ (distinguishable) spin- $1 / 2$ particles and it can be decomposed in terms of eigenspaces of total angular momentum. More precisely consider

$$
L_{k}=\frac{1}{2} \sum_{j} \sigma_{k}^{(j)}, k=1,2,3
$$

the $k$-component of total angular momentum (i.e. $\sigma_{k}$ is the $k^{\text {th }}$ Pauli matrix and $\sigma^{(j)} \in \mathcal{B}\left(\mathcal{H}^{\otimes N}\right)$ is defined according to Equation (7.1)) and $\vec{L}^{2}=\sum_{k} L_{k}^{2}$. The eigenvalue expansion of $\vec{L}^{2}$ is well known to be

$$
\vec{L}=\sum_{j} s(s+1) P_{s}, \text { with } s= \begin{cases}0,1, \ldots, N / 2 & N \text { even } \\ 1 / 2,3 / 2, \ldots, N / 2 & N \text { odd }\end{cases}
$$

where the $P_{s}$ denote the projections to the eigenspaces of $\vec{L}^{2}$. It is easy to see that both representations $U \mapsto U^{\otimes N}$ and $p \mapsto V_{p}$ commute with $\vec{L}$. Hence the eigenspaces $P_{s} \mathcal{H}^{\otimes N}$ of $\vec{L}^{2}$ are invariant subspaces of $U^{\otimes N}$ and $V_{p}$ and this implies that the restriction of $U^{\otimes N}$ and $V_{p}$ to them are representations of SU(2) respectively $\mathrm{S}_{N}$. Since $\vec{L}^{2}$ is constant on $P_{s} \mathcal{H}^{\otimes N}$ the $\mathrm{SU}(2)$ representation we get in this way must be (naturally isomorphic to) a multiple of the irreducible spin- $s$ representation $\pi_{s}$. It is defined by

$$
\pi_{s}\left[\exp \left(\frac{i}{2} \sigma_{k}\right)\right]=\exp \left(i L_{k}^{(s)}\right) \text { with } L_{k}^{(s)}=\frac{1}{2} \sum_{j=1}^{2 s} \sigma_{k}^{(j)},
$$

on the representation space

$$
\mathcal{H}_{s}=\mathcal{H}_{+}^{\otimes 2 s}
$$

(the Bose-subspace of $\mathcal{H}^{\otimes 2 s}$ ). Hence we get

$$
P_{s} \mathcal{H}^{\otimes N} \cong \mathcal{H}_{s} \otimes \mathcal{K}_{N, s}, \quad U^{\otimes N} \psi=\left(\pi_{s}(U) \otimes \mathbb{I}\right) \psi \forall \psi \in P_{s} \mathcal{H}^{\otimes N} .
$$

Since $V_{p}$ and $U^{\otimes N}$ commute the Hilbert space $\mathcal{K}_{N, s}$ carries a representation $\widehat{\pi}_{N, s}(p)$ of $\mathrm{S}_{N}$ which is irreducible as well. Note that $\mathcal{K}_{N, s}$ depends in contrast to $\mathcal{H}_{s}$ on the number $N$ of tensor factors and its dimension is (see [100 or 142 for general $d$ )

$$
\operatorname{dim} \mathcal{K}_{N, s}=\frac{2 s+1}{N / 2+s+1}\left(\begin{array}{c}
N \\
N / 2-s
\end{array}\right) \text {. }
$$


Summarizing the discussion we get

$$
\mathcal{H}^{\otimes N} \cong \bigoplus_{s} \mathcal{H}_{s} \otimes \mathcal{K}_{N, s}, U^{\otimes N} \cong \bigoplus_{s} \pi_{s}(U) \otimes \mathbb{I}, V_{p} \cong \bigoplus_{s} \mathbb{I} \otimes \widehat{\pi}(p) .
$$

Let us consider now a fully symmetric operation $T$. Permutation invariance $\left(\alpha_{p}(T)=T\right.$ and $\left.\beta_{p}(T)=T\right)$ implies together with Equation (7.26) that

$$
T\left(A_{j} \otimes B_{j}\right)=\bigoplus_{s}\left[\frac{\operatorname{tr}\left(B_{j}\right)}{\operatorname{dim} \mathcal{K}_{N, j}} T_{s j}\left(A_{j}\right) \otimes \mathbb{I}\right] \text { with } T_{s j}: \mathcal{B}\left(\mathcal{H}_{j}\right) \rightarrow \mathcal{B}\left(\mathcal{H}_{s}\right),
$$

holds if $A_{j} \otimes B_{j} \in \mathcal{B}\left(\mathcal{H}_{j} \otimes \mathcal{K}_{N, j}\right)$. The operations $T_{s j}$ are unital and have, according to $\gamma_{U}(T)=T$ the following covariance properties

$$
\pi_{s}(U) T\left(A_{j}\right) \pi_{s}\left(U^{*}\right)=T\left[\pi_{j}(U) A_{j} \pi_{j}\left(U^{*}\right)\right] \forall U \in \mathrm{SU}(2) .
$$

The classification of all fully symmetric channels $T$ is reduced therefore to the study of all these $T_{s j}$.

We can apply now the covariant version of Stinespring's theorem (Theorem 3.2.2) to find that

$$
T_{s j}\left(A_{j}\right)=V^{*}\left(A_{j} \otimes \mathbb{I}\right) V, V: \mathcal{H}_{s} \rightarrow \mathcal{H}_{j} \otimes \widetilde{\mathcal{H}}, V \pi_{s}(U)=\pi_{j}(U) \otimes \widetilde{\pi}(U) V,
$$

where $\tilde{\pi}$ is a representation of $\mathrm{SU}(2)$ on $\widetilde{\mathcal{H}}$. If $\widetilde{\pi}$ is irreducible with total angular momentum $l$ the "intertwining operator" $V$ is well known: Its components in a particularly chosen basis concide with certain Clebsh-Gordon coefficients. Hence the corresponding operation is uniquely determined (up to unitary equivalence) and we write

$$
T_{s j l}\left(A_{j}\right)=\left[V_{l}\left(A_{j} \otimes \mathbb{I}\right) V_{l}\right], \quad V_{l} \pi_{s}(U)=\pi_{j}(U) \otimes \pi_{l}(U) V_{l}
$$

where $l$ can range from $|j-s|$ to $j+s$. Since a general representation $\widetilde{\pi}$ can be decomposed into irreducible components we see that each covariant $T_{s j}$ is a convex linear combination of the $T_{s j l}$ and we get with Equation (7.27)

$$
T\left(A_{j} \otimes B_{j}\right)=\bigoplus_{s}\left[\sum_{l} c_{j l}\left[T_{s j l}\left(A_{j}\right) \otimes\left(\operatorname{tr}\left(B_{j}\right) \mathbb{I}\right)\right]\right]
$$

where the $c_{j l}$ are constrained by $c_{j l}>0$ and $\sum_{j} c_{j l}=\left(\operatorname{dim} \mathcal{K}_{N, j}\right)^{-1}$. In this way we have parameterized the set of fully symmetric operations completely in terms of group theoretical data and we can rewrite $\mathcal{F}_{\#, \natural}(T)$ accordingly. This leads to an optimization problem for a quantity depending only on $s, j$ and $l$, which is at least in some cases solvable.

To generalize the scheme just presented to the case $\mathcal{H}=\mathbb{C}^{d}$ with arbitrary $d$ we only have to find a replacement for the decomposition in Equation (7.26). This, however, is well known from group theory:

$$
\mathcal{H}^{\otimes N} \cong \bigoplus_{Y} \mathcal{H}_{Y} \otimes \mathcal{K}_{Y}, U^{\otimes N} \cong \bigoplus_{Y} \pi_{Y}(U) \otimes \mathbb{I}, V_{p} \cong \bigoplus_{Y} \mathbb{I} \otimes \widehat{\pi}_{Y}(p)
$$

where $\pi_{Y}: \mathrm{U}(d) \rightarrow \mathcal{B}\left(\mathcal{H}_{Y}\right)$ and $\widehat{\pi}_{Y}: \mathrm{S}_{N} \rightarrow \mathcal{B}\left(\mathcal{K}_{Y}\right)$ are irreducible representations. The summation index $Y$ runs over all Young frames with $d$ rows and $N$ boxes, i.e., by the arrangements of $N$ boxes into $d$ rows of lengths $Y_{1} \geq Y_{2} \geq \cdots \geq Y_{d} \geq 0$ with $\sum_{k} Y_{k}=N$. The relation to total angular momentum $s$ used as the parameter for $d=2$ is given by $Y_{1}-Y_{2}=2 s$, which determines $Y$ together with $Y_{1}+Y_{2}=N$ completely. The rest of the arguments applies without significant changes, this is in 
particular the case for Equation (7.31) which holds for general $d$ if we replace $s, j$ and $l$ by Young frames. However, the representation theory of $\mathrm{U}(d)$ becomes much more difficult. The generalization of results available for qubits $(d=2)$ to $d>2$ is therefore by no means straightforward.

Finally let us give a short comment on Gaussian states here. Obviously the methods just described do not apply in this case. However, we can consider instead of $U^{\otimes N}$-covariance, covariance with respect to phase-space translations. Following this idea some results concerning optimal cloning of Gaussian states are obtained (see [43] and the refences therein), but the corresponding general theory is not as far developed as in the finite dimensional case.

7.1.4. Distillation of entanglement. - Finally let us have another look at distillation of entanglement. The basic idea is quite the same as for optimal cloning: Use multiple inputs to approximate a task which is impossible with one-shot operations. From a more technical point of view, however, it does not fit into the general scheme proposed up to now. Nevertheless, some of the arguments can be adopted in an easy way. First of all we have to replace the "one-particle" Hilbert space $\mathcal{H}$ with a two-fold tensor product $\mathcal{H}_{A} \otimes \mathcal{H}_{B}$ and the channels we have to look at are LOCC operations

$$
T: \mathcal{B}\left(\mathcal{H}_{A}^{\otimes M} \otimes \mathcal{H}_{B}^{\otimes M}\right) \rightarrow \mathcal{B}\left(\mathcal{H}_{A}^{\otimes N} \otimes \mathcal{H}_{B}^{\otimes N}\right) ;
$$

cf. Section 4.3. Our aim is to determine $T$ such that $T^{*}\left(\rho^{\otimes N}\right)$ is for each distillable (mixed) state $\rho \in \mathcal{B}^{*}\left(\mathcal{H}_{A} \otimes \mathcal{H}_{B}\right)$, close to the $M$-fold tensor product $|\Psi\rangle\left\langle\left.\Psi\right|^{\otimes M}\right.$ of a maximally entangled state $\Psi \in \mathcal{H}_{A} \otimes \mathcal{H}_{B}$. A figure of merit with a similar structure as the $\mathcal{F}_{\# \text {,all }}$ studied above can be derived directly from the definition of the entanglement measure $E_{D}$ in Section 5.1.3: We define (replacing the trace-norm distance with a fidelity)

$$
\mathcal{F}_{D}(T)=\inf _{\rho} \inf _{\Psi}\left\langle\Psi^{\otimes M}, T^{*}\left(\rho^{\otimes N}\right) \Psi^{\otimes M}\right\rangle
$$

where the infima are taken over all maximally entangled states $\Psi$ and all distillable states $\rho$. Alternatively we can look at state dependent measures, which seem to be particularly important if we try to calculate $E_{D}(\rho)$ for some state $\rho$. In this case we simply get

$$
\mathcal{F}_{D, \rho}(T)=\inf _{\Psi}\left\langle\Psi^{\otimes M}, T^{*}\left(\rho^{\otimes N}\right) \Psi^{\otimes M}\right\rangle .
$$

To translate the group theoretical analysis of the last two subsections is somewhat more difficult. As in the case of $\mathcal{F}_{\#, \downarrow}$ we can restrict the search for optimizers to permutation invariant operations, i.e. $\alpha_{p}(T)=T$ and $\beta_{p}(T)=T$ in the terminology of Subsection 7.1.2. Unitary covariance

$$
U^{\otimes N} T(A) U^{* \otimes N}=T\left(U^{\otimes M} A U^{* \otimes M}\right)
$$

however, can not be assumed for all unitaries $U$ of $\mathcal{H}_{A} \otimes \mathcal{H}_{B}$, but only for local ones $\left(U=U_{A} \otimes U_{B}\right)$ in the case of $\mathcal{F}_{D}$ or only for local $U$ which leave $\rho$ invariant for $\mathcal{F}_{D, \rho}$. This makes the analogon of the decomposition scheme from Subsection 7.1.3 more difficult and such a study is (up to my knowledge) not yet done. A related subproblem arises if we consider $\mathcal{F}_{D, \rho}$ from Equation (7.35) for a state $\rho$ with special symmetry properties; e.g. an OO-invariant state. The corresponding optimization might be simpler and a solution would be relevant for the calculation of $E_{D}$.

\subsection{Optimal devices}

Now we can consider the optimization problems associated to the figures of merit discussed in the last section. This means that we are searching for those devices 
which approximate the impossible tasks in question in the best possible way. As pointed out at the beginning of this Chapter this can be done for finite $N$ and in the limit $N \rightarrow \infty$. The latter is postponed to the next section.

7.2.1. Optimal cloning. - The quality of an optimal, pure state cloner is defined by the figures of merit $\mathcal{F}_{c, \#}$ in Equations (7.2) and (7.3) and the group theoretic ideas sketched in Subsection 7.1.3 allow the complete solution of this problem. We will demonstrate some of the basic ideas in the qubit case first and state the final result afterwards in full generality.

The solvability of this problem relies in part on the special structure of the figures of merit $\mathcal{F}_{c, \#}$, which allows further simplifications of the general scheme sketched in Subsection 7.1.3. If we consider e.g. $\mathcal{F}_{c, 1}(T)$ (the other case works similarly) we get:

$$
\begin{aligned}
\mathcal{F}_{c, 1}(T) & =\inf _{j=1, \ldots, N} \inf _{\sigma \operatorname{pure}} \operatorname{tr}\left(\sigma^{(j)} T^{*}\left(\sigma^{\otimes N}\right)\right) \\
& \left.=\inf _{j=1, \ldots, N} \inf _{\sigma \operatorname{pure}} \operatorname{tr}\left(T\left(\sigma^{(j)}\right) \sigma^{\otimes N}\right)\right) \\
& =\inf _{j=1, \ldots, N} \inf _{\psi}\left\langle\psi^{\otimes N}, T\left(\sigma^{(j)}\right) \psi^{\otimes N}\right\rangle .
\end{aligned}
$$

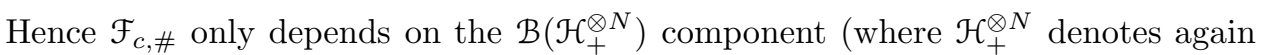
the Bose-subspace of $\mathcal{H}^{\otimes N}$ ) of $T$ and we can assume without loss of generality that $T$ is of the form

$$
T: \mathcal{B}\left(\mathcal{H}^{\otimes M}\right) \rightarrow \mathcal{B}\left(\mathcal{H}_{+}^{\otimes N}\right) .
$$

The restriction of $U^{\otimes N}$ to $\mathcal{H}_{+}^{\otimes N}$ is an irreducible representation (for any $d$ ) and in the qubit case $(d=2)$ we have $U^{\otimes N} \psi=\pi_{s}(U) \psi$ with $s=N / 2$ for all $\psi \in \mathcal{H}_{+}^{\otimes N}$. The decomposition of $T$ from Equation (7.27) contains therefore only those summands with $s=N / 2$. This simplifies the optimization problem significantly, since the number of variables needed to parametrize all relevant cloning maps according to Equation (7.31) is reduced from 3 to 2 . A more detailed (and non-trivial) analysis shows that the maximum for $\mathcal{F}_{c, 1}$ and $\mathcal{F}_{c \text {,all }}$ is attained if all terms in (7.31) except the one with $s=N / 2, j=N / 2$ and $l=(M-N) / 2$ vanish. The precise result is stated in the following theorem (668, 31, 32] for qubits and [167, 98 for general $d$ ).

TheOREM 7.2.1 For each $\mathcal{H}=\mathbb{C}^{d}$ both figures of merit $\mathcal{F}_{c, 1}$ and $\mathcal{F}_{c \text {,all }}$ are maximized by the cloner

$$
\widehat{T}^{*}(\rho)=\frac{d[N]}{d[M]} S_{M}(\rho \otimes \mathbb{I}) S_{M}
$$

where $d[N], d[M]$ denote the dimensions of the symmetric tensor products $\mathcal{H}_{+}^{\otimes N}$ respectively $\mathcal{H}_{+}^{\otimes M}$ and $S_{M}$ is the projection from $\mathcal{H}^{\otimes M}$ to $\mathcal{H}_{+}^{\otimes M}$. This implies for the optimal fidelities

$$
\mathcal{F}_{c, 1}(N, M)=\frac{d-1}{d} \frac{N}{N+d} \frac{M+d}{M}
$$

and

$$
\mathcal{F}_{c, \text { all }}(N, M)=\frac{d[N]}{d[M]}
$$

$\widehat{T}$ is the unique solution for both optimization problems, i.e. there is no other operation $T$ of the form (7.40) which maximizes $\mathcal{F}_{c, 1}$ or $\mathcal{F}_{c \text {,all }}$. 
There are two aspects of this result which deserve special attention. One is the relation to state estimation which is postponed to Subsection 7.2.3. The second concerns the role of correlations: It does not matter whether we are looking for the quality of each single clone $\left(\mathcal{F}_{c, 1}\right)$ only, or whether correlations are taken into account $\left(\mathcal{F}_{c \text {,all }}\right)$. In both cases we get the same optimal solution. This is a special feature of pure states, however. Although there are no concrete results for quantum systems, it can be checked quite easily in the classical case that considering correlations changes the optimal cloner for arbitrary mixed states drastically.

7.2.2. Purification. - To find an optimal purification device, i.e. maximizing $\mathcal{F}_{R \text {,\# }}$, is more difficult then the cloning problem, because the simplification from Equation (7.40) does not apply. Hence we have to consider all the summands in the direct sum decomposition of $T$ from Equation (7.31) and solutions are available only for qubits. Therefore we will assume for the rest of this subsection that $\mathcal{H}=\mathbb{C}^{2}$ holds. The $\mathrm{SU}(2)$ symmetry of the problem allows us to assume without loss of generality that the pure initial state $\psi$ coincides with one of the basis vectors. Hence we get for the (noisy) input states of the purifier

$$
\begin{aligned}
\rho(\beta) & =\frac{1}{2 \cosh (\beta)} \exp \left(2 \beta \frac{\sigma_{3}}{2}\right)=\frac{1}{e^{\beta}+e^{-\beta}}\left(\begin{array}{ll}
e^{\beta} & 0 \\
0 & e^{-\beta}
\end{array}\right) \\
& =\tanh (\beta)|\psi\rangle\left\langle\psi\left|+(1-\tanh (\beta)) \frac{1}{2} \mathbb{I}, \quad \psi=\right| 0\right\rangle
\end{aligned}
$$

The parameterization of $\rho$ in terms of the "pseudo-temperature" $\beta$ is chosen here, because it simplifies some calculations significantly (as we will see soon). The relation to the form of $\rho=R^{*} \sigma$ initially given in Equation (7.4) is obviously $\vartheta=\tanh (\beta)$.

To state the main result of this subsection we have to decompose the product state $\rho(\beta)^{\otimes N}$ into spin- $s$ components. This can be done in terms of Equation (7.26). $\rho(\beta)$ is not unitary of course. However we can apply $(7.26)$ by analytic continuation, i.e. we treat $\rho(\beta)$ in the same way as we would $\exp \left(i \beta \sigma_{3}\right)$. It is then straightforward

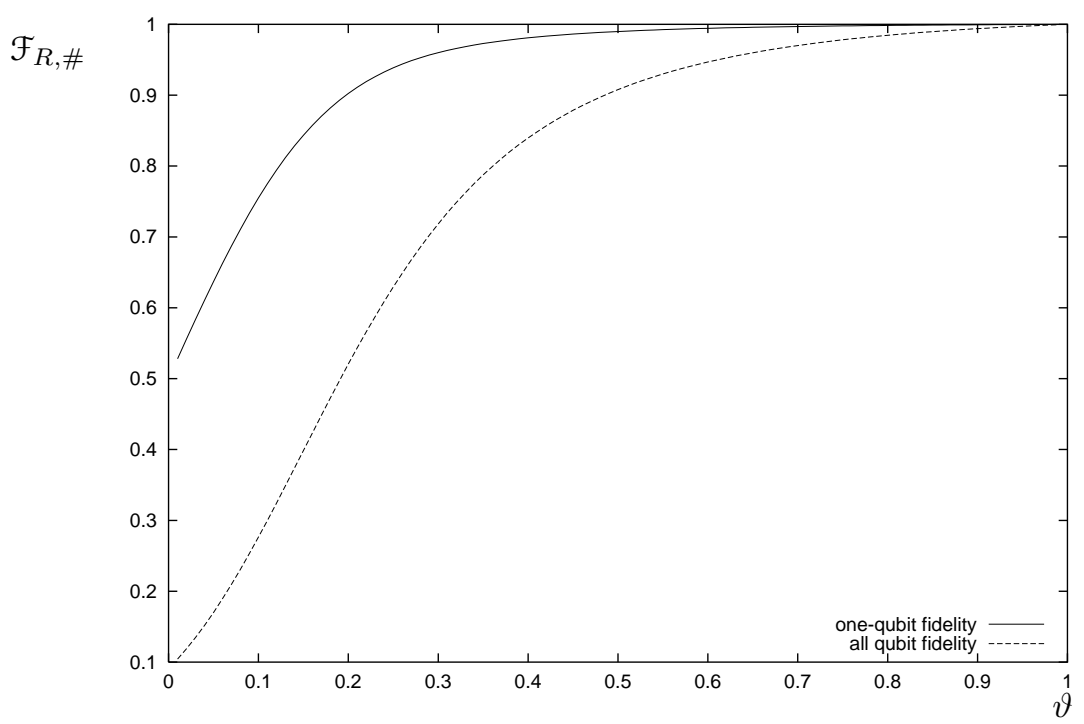

Figure 7.1: One- and all-qubit fidelities of the optimal purifier for $N=100$ and $M=10$. Plotted as a function of the noise parameter $\vartheta$. 
to get

$$
\rho(\beta)^{\otimes N}=\bigoplus_{s} w_{N}(s) \rho_{s}(\beta) \otimes \frac{\mathbb{I}}{\operatorname{dim} \mathcal{K}_{N, s}}
$$

with

$$
w_{N}(s)=\frac{\sinh ((2 s+1) \beta)}{\sinh (\beta)(2 \cosh (\beta))^{N}} \operatorname{dim} \mathcal{K}_{N, s},
$$

and

$$
\rho_{s}(\beta)=\frac{\sinh (\beta)}{\sinh ((2 s+1) \beta)} \exp \left(2 \beta L_{3}^{(s)}\right)
$$

where $L_{3}^{(s)}$ is the 3-component of angular momentum in the spin-s representation and the dimension of $\mathcal{K}_{N, s}$ is given in Equation (7.25). By (7.23) the representation space of $\pi_{s}$ coincides with the symmetric tensor product $\mathcal{H}_{+}^{2 s}$. Hence we can interpret $\rho_{s}(\beta)$ as a state of $2 s$ (indistinguishable) particles. In other words the decomposition of $\rho(\beta)^{\otimes N}$ leads in a natural way to a family of operations

$$
Q_{s}: \mathcal{B}\left(\mathcal{H}_{+}^{\otimes 2 s}\right) \rightarrow \mathcal{B}\left(\mathcal{H}^{\otimes N}\right) \text {, with } Q_{s}^{*}\left[\rho(\beta)^{\otimes N}\right]=\rho_{s}(\beta) .
$$

We can think of the family $Q_{s}$, of operations as an instrument $Q$ which measures the number of output systems and transforms $\rho(\beta)^{\otimes N}$ to the appropriate $\rho_{s}(\beta)$. The crucial point is now that the purity of $\rho_{s}(\beta)$, measured in terms of fidelities with respect to $\psi$ increases provided $s>1 / 2$ holds. Hence we can think of $Q$ as a purifier which arises naturally by reduction to irreducible spin components [46. Unfortunately $Q$ does not produce a fixed number of output systems. The most obvious way to construct a device which produces always the same number $M$ of outputs is to run the optimal $2 s \rightarrow M$ cloner $\widehat{T}_{2 s \rightarrow M}$ if $2 s<M$ or to drop $2 s-M$ particles if $M \leq 2 s$ holds. More precisely we can define $\widehat{Q}: \mathcal{B}\left(\mathcal{H}^{\otimes M}\right) \rightarrow \mathcal{B}\left(\mathcal{H}^{\otimes N}\right)$ by

$$
\widehat{Q}^{*}\left[\rho(\beta)^{\otimes N}\right]=\sum_{s} w_{N}(s) \widehat{T}_{2 s \rightarrow M}^{*}\left[\rho_{s}(\beta)\right],
$$

with

$$
\widehat{T}_{2 s \rightarrow M}^{*}(\rho)= \begin{cases}\frac{d[2 s]}{d[M]} S_{M}(\rho \otimes \mathbb{I}) S_{M} & \text { for } M>2 s \\ \operatorname{tr}_{2 s-M} \rho & \text { for } M \leq 2 s .\end{cases}
$$

$\operatorname{tr}_{2 s-M}$ denotes here the partial trace over the $2 s-M$ first tensor factors. Applying the general scheme of Subsection 7.1.3 shows that this is the best way to get exactly $M$ purified qubits [100:

Theorem 7.2.2 The operation $\widehat{Q}$ defined in Equation (7.49) maximizes $\mathcal{F}_{R, 1}$ and $\mathcal{F}_{R \text {,all }}$. It is called therefore the optimal purifier. The maximal values for $\mathcal{F}_{R, 1}$ and $\mathcal{F}_{R \text {,all }}$ are given by

$$
\mathcal{F}_{R, 1}(N, M)=\sum_{s} w_{N}(s) f_{1}(M, \beta, s), \mathcal{F}_{R, \text { all }}(N, M)=\sum_{s} w_{N}(s) f_{\text {all }}(M, \beta, s)
$$

with

$$
\begin{aligned}
& 2 f_{1}(M, \beta, s)-1= \\
& \quad= \begin{cases}\frac{2 s+1}{2 s} \operatorname{coth}((2 s+1) \beta)-\frac{1}{2 s} \operatorname{coth} \beta & \text { for } 2 s>M \\
\frac{1}{2 s+2} \frac{M+2}{M}((2 s+1) \operatorname{coth}((2 s+1) \beta)-\operatorname{coth} \beta) & \text { for } 2 s \leq M .\end{cases}
\end{aligned}
$$




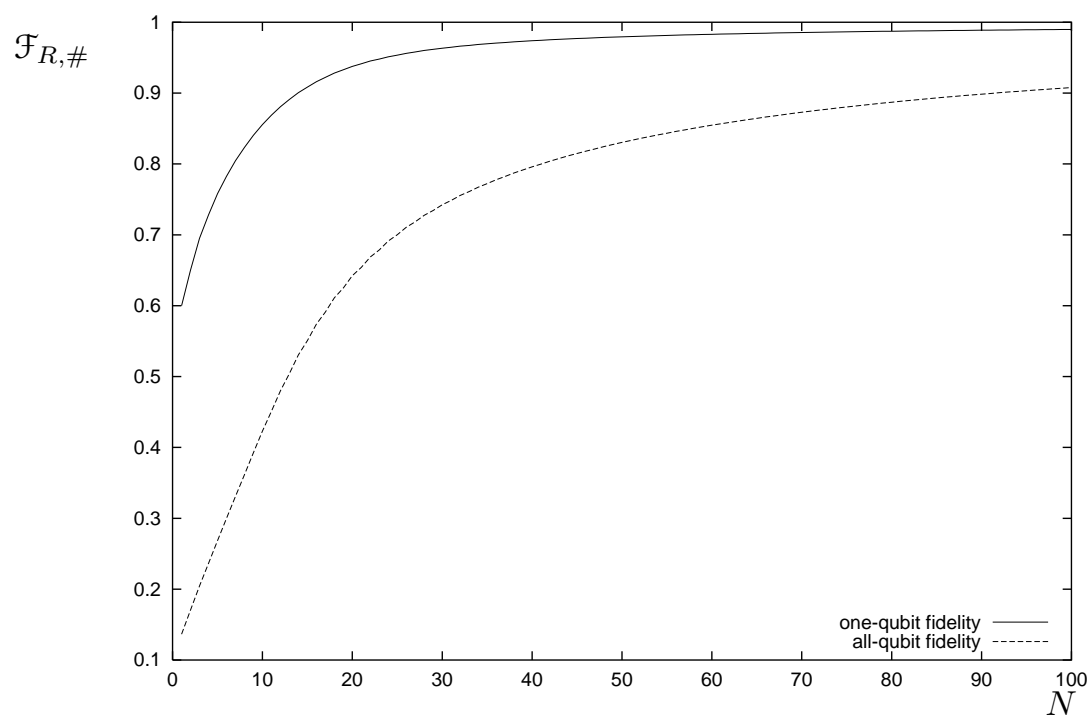

Figure 7.2: One- and all-qubit fidelities of the optimal purifier for $\vartheta=0.5$ and $M=10$. Plotted as a function of $N$.

and

$$
f_{\text {all }}(M, \beta, s)= \begin{cases}\frac{2 s+1}{M+1} \frac{1-e^{-2 \beta}}{1-e^{-(4 s+2) \beta}} & M \leq 2 s \\
\frac{1-e^{-2 \beta}}{1-e^{-(4 s+2) \beta}}\left(\begin{array}{c}
2 s \\
M
\end{array}\right)^{-1} \sum_{K}\left(\begin{array}{l}
K \\
M
\end{array}\right) e^{2 \beta(K-s)} & M>2 s .\end{cases}
$$

The expression for the optimal fidelities given here look rather complicated and are not very illuminating. We have plotted there both quantities as a function of $\vartheta$ (Figure 7.1) of $N$ (Figure 7.2) and $M$ (Figure 7.3). While the first two plots looks quite similar the functional behavior in dependence of $M$ seems to be very different. The study of the asymptotic behavior in the next Section will give a precise analysis of this observation.

7.2.3. Estimating pure states. - We have already seen in Section 4.2 that the cloning problem and state estimation are closely related, because we can construct an approximate cloner $T$ from an estimator $E$ simply by running $E$ on the $N$ input states, and preparing $M$ systems according to the attained classical information. In this section we want to go the other way round and show that the optimal cloner derived in Theorem 7.2.1 leads immediately to an optimal pure state estimator; cf. 33.

To this end let us assume that $E$ has the form (cf. Section 4.2)

$$
\mathcal{C}(X) \ni f \mapsto E(f)=\sum_{\sigma \in X} f(\sigma) E_{\sigma} \in \mathcal{B}\left(\mathcal{H}^{\otimes N}\right)
$$

where $X \subset \mathcal{B}^{*}(\mathcal{H})$ is a finite set $\prod^{\natural}$ of pure states. The quality of $E$ can be measured

\footnotetext{
${ }^{1}$ The generalization of the following considerations to continuous sets and a measure theoretic setup is straightforward and does not lead to a different result; i.e. we can not improve the estimation quality with continuous observables.
} 


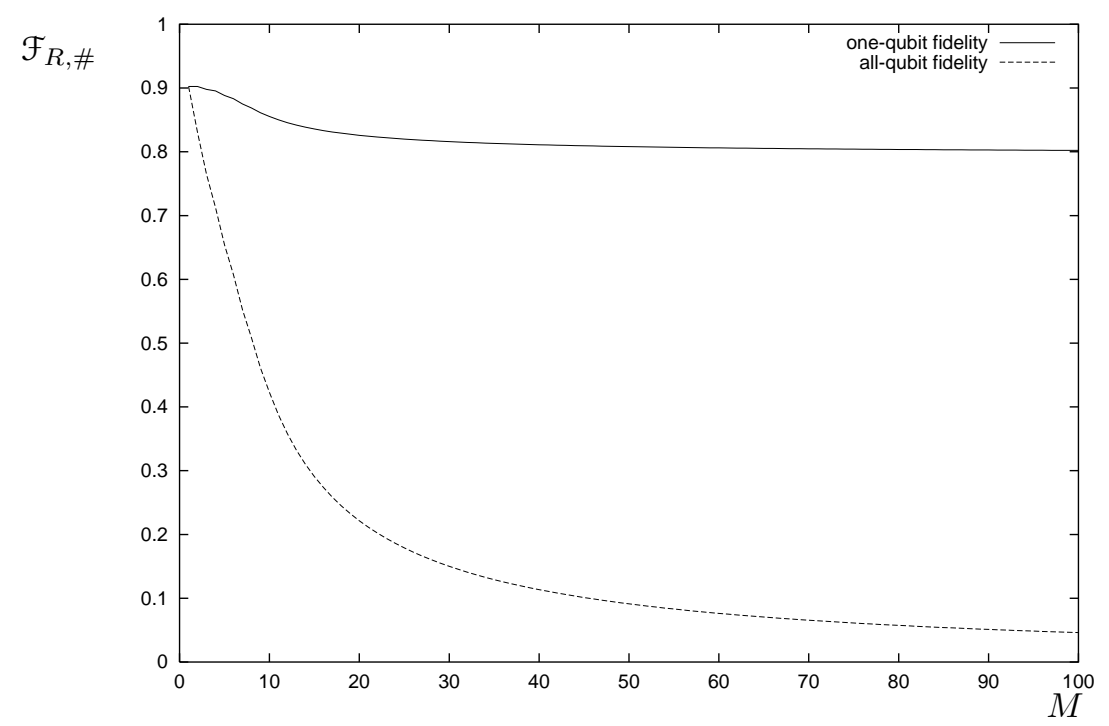

Figure 7.3: One- and all-qubit fidelities of the optimal purifier for $\vartheta=0.5$ and $N=10$. Plotted as a function of $M$.

in analogy to Subsection 7.1.1 by a fidelity-like quantity

$$
\mathcal{F}_{s}(E)=\inf _{\psi \in \mathcal{H}}\left\langle\psi, \rho_{\psi} \psi\right\rangle=\inf _{\psi \in \mathcal{H}} \sum_{\sigma \in X}\left\langle\psi^{\otimes N}, E_{\sigma} \psi^{\otimes N}\right\rangle\langle\psi, \sigma \psi\rangle
$$

where $\rho_{\psi}=\sum_{\sigma}\left\langle\psi^{\otimes N}, E_{\sigma} \psi^{\otimes n}\right\rangle \sigma$ is the (density matrix valued) expectation value of $E$ and the infimum is taken over all pure states $\psi$. Hence $\mathcal{F}_{s}(E)$ measures the worst fidelity of $\rho_{\psi}$ with respect to the input state $\psi$. If we construct now a cloner $T_{E}$ from $E$ by

$$
T_{E}^{*}\left(|\psi\rangle\left\langle\left.\psi\right|^{\otimes N}\right)=\sum_{\sigma}\left\langle\psi^{\otimes N}, E_{\sigma} \psi^{\otimes n}\right\rangle \sigma^{\otimes M}\right.
$$

its one-particle fidelity $\mathcal{F}_{c, 1}\left(T_{E}\right)$ coincides obviously with $\mathcal{F}_{s}(E)$. Since we can produce in this way arbitrary many clones of the same quality we see that $\mathcal{F}_{s}(E)$ is smaller than $\mathcal{F}_{c, 1}(N, M)$ for all $M$ and therefore

$$
\mathcal{F}_{s}(E) \leq \mathcal{F}_{c, 1}(N, \infty)=\lim _{M \rightarrow \infty} \mathcal{F}_{c, 1}(N, M)=\frac{d-1}{d} \frac{N}{N+d}
$$

where we can look at $\mathcal{F}_{c, 1}(N, \infty)$ as the optimal quality of a cloner which produces arbitrary many outputs from $N$ input systems.

To see that this bound can be saturated consider an asymptotically exact family

$$
\mathcal{C}\left(X_{M}\right) \ni f \mapsto E^{M}(f)=\sum_{\sigma \in X} f(\sigma) E_{\sigma}^{M} \in \mathcal{B}\left(\mathcal{H}^{\otimes M}\right), X_{M} \subset \mathcal{S}(\mathcal{H})
$$

of estimators, i.e. the error probabilities (4.17) vanish in the limit $N \rightarrow \infty$. If the $E_{\sigma}^{M} \in \mathcal{B}\left(\mathcal{H}^{\otimes M}\right)$ are pure tensor products (i.e. the $E^{M}$ are realized by a "quorum" of observables as described in Subsection 4.2.1) they can not distinguish between the output state $\widehat{T}^{*}\left(\rho^{\otimes N}\right)$ (which is highly correlated) and the pure product state $\widetilde{\rho}^{\otimes M}$ where $\widetilde{\rho} \in \mathcal{B}^{*}(\mathcal{H})$ denotes the partial trace over $M-1$ tensor factors (due to permutation invariance it does not matter which factors we trace away here). 
Hence if we apply $E^{M}$ to the output of the optimal $N$ to $M$ cloner $\widehat{T}_{N \rightarrow M}$ we get an estimate for $\widetilde{\rho}$ and in the limit $M \rightarrow \infty$ this estimate is exact. The fidelity $\langle\psi, \widetilde{\rho} \psi\rangle$ of $\widetilde{\rho}$ with respect to the pure input state $\psi$ of $\widehat{T}_{N \rightarrow M}$ coincides however with $\mathcal{F}_{c, 1}(N, M)$. Hence the composition of $\widehat{T}_{N \rightarrow M}$ with $E^{M}$ converges 2 to an estimator $E$ with $\mathcal{F}_{e}(E)=\mathcal{F}_{c, 1}(N, \infty)$. We can rephrase this result roughly in the from: "producing infinitely many optimal clones of a pure state $\psi$ is the same as estimating $\psi$ optimally".

7.2.4. The UNOT gate. - The discussion of the last subsection shows that the optimal cloner $\widehat{T}_{N \rightarrow M}$ produces better clones then any estimation based scheme (as in Equation (7.56)), as long as we are interested only in finitely many copies. Loosely speaking we can say that the detour via classical information is wasteful and destroys too much quantum information. The same is true for the optimal purifier: We can first run an estimator on the mixed input state $\rho(\beta)^{\otimes N}$, apply the inverse $\left(R^{*}\right)^{-1}$ of the channel map to the attained classical data and reprepare arbitrary many purified qubits accordingly. The quality of output systems attained this way is, however worse, than those of the optimal purifier from Equation (7.49) as long as the number $M$ of output systems is finite; this can be seen easily from Figure 7.3. In this sense the UNOT gate is a harder task than cloning and purification, because there is no quantum operation which performs better than the estimation based strategy. The following theorem can be proved again with the group theoretical scheme from Subsection 7.1.3 [36].

TheOrem 7.2.3 Let $\mathcal{H}=\mathbb{C}^{2}$. Among all channels $T: \mathcal{B}(\mathcal{H}) \rightarrow \mathcal{B}\left(\mathcal{H}_{+}^{\otimes N}\right)$ the estimation based scheme just described attains the biggest possible value for the fidelity $\mathcal{F}_{\theta, \#}$, namely

$$
\mathcal{F}_{\theta, 1}(N, 1)=\mathcal{F}_{\theta, \text { all }}(N, 1)=1-\frac{1}{N+2} .
$$

The dependence on the number $M$ of outputs is not interesting here,. because the optimal device produces arbitrary many copies of the same quality.

\subsection{Asymptotic behaviour}

If a device, such as the optimal cloner, is given which produces $M$ output system from $N$ inputs it is interesting to ask for the maximal rate, i.e. the maximal ratio $M(N) / N$ in the limt $N \rightarrow \infty$ such that the asymptotic fidelity $\lim _{N \rightarrow \infty} \mathcal{F}(N, M(N))$ is above a certain threshold (preferably equal to one). Note that this type of question was very important as well for distillation of entanglement and channel capacities, but almost not computable in there. In the current context this type of question is somewhat easier to answer. This relies on the one hand on the group theoretical structure presented in the last section and on the other on the close relation to quantum state estimation. We start this section therefore with a look on some aspects of the asymptotics of mixed state estimation.

7.3.1. Estimating mixed state. - If we do not know a priori that the input systems are in a pure state much less is known about estimating and cloning. It is in particular almost impossible to say anything about optimality for finitely many input systems (only if $N$ is very small e.g. 156]). Nevertheless some strong results are available for the behavior in the limit $N \rightarrow \infty$ and we will give here a short review of some of them.

One quantity, interesting to be analyzed for a family of estimators $E^{N}$ in the limit $N \rightarrow \infty$ is the variance of the $E^{N}$. To state some results in this context it is convenient to parameterize the state space $\mathcal{S}(\mathcal{H})$ or parts of it in terms of $n$ real

\footnotetext{
${ }^{2}$ Basically convergence must be shown here. It follows however easily from the corresponding property of the $E^{M}$.
} 
parameters $x=\left(x_{1}, \ldots, x_{n}\right)=\Sigma \subset \mathbb{R}^{n}$ and to write $\rho(x)$ as the corresponding state. If we want to cover all states, one particular parameterization is e.g. the generalized Bloch ball from Subsection 2.1.2. An estimator taking $N$ input systems is now a (discrete) observable $\left.E_{x}^{N} \in \mathcal{\mathcal { B } ( \mathcal { H } ^ { \otimes }}{ }^{N}\right), x \in X_{N}$ with values in a (finite) subset $X_{N}$ of $\Sigma$. The expectation value of $E^{N}$ in the state $\rho(x)^{\otimes N}$ is therefore the vector $\left\langle E^{N}\right\rangle_{x}$ with components $\left\langle E^{N}\right\rangle_{x}, j, j=1, \ldots, n$ given by

$$
\left\langle E^{N}\right\rangle_{x, j}=\sum_{y \in X_{N}} y_{j} \operatorname{tr}\left(E_{y}^{N} \rho(x)^{\otimes N}\right)
$$

and the mean quadratic error is described by the matrix

$$
V_{j k}^{N}(x)=\sum_{y \in X_{N}}\left(\left\langle E_{N}\right\rangle_{x, j}-y_{j}\right)\left(\left\langle E_{N}\right\rangle_{x, k}-y_{k}\right) \operatorname{tr}\left(E_{y}^{N} \rho(x)^{\otimes N}\right) .
$$

For a good estimation strategy we expect that $V_{j k}(x)$ decreases as $1 / N$, i.e.

$$
V_{j k}^{N}(x) \simeq \frac{W_{j k}(x)}{N}
$$

where the scaled mean quadratic error matrix $W_{j k}(x)$ does not depend on $N$. The task is now to find bounds on this matrix. We will state here two results taken from [66. To this end we need the Hellström quantum information matrix

$$
H_{j k}(x)=\operatorname{tr}\left[\rho(x) \frac{\lambda j(x) \lambda_{k}(x)-\lambda_{k}(x) \lambda_{j}(x)}{2}\right]
$$

which is defined in terms of symmetric logarithmic derivatives $\lambda_{j}$, which in turn are implicitly given by

$$
\frac{\partial \rho(x)}{\partial x_{j}}=\frac{\lambda_{j}(x) \rho(x)+\rho(x) \lambda_{j}(x)}{2} .
$$

Now we have the following theorem 66]:

THEOREM 7.3.1 Consider a family of estimators $E^{N}, N \in \mathbb{N}$ as described above such that the following conditions hold:

1. The scaled mean quadratic error matrix $N V_{j k}^{N}(x)$ converges uniformly in $x$ to $W_{j k}(x)$ as $N \rightarrow \infty$.

2. $W_{j k}(x)$ is continuous at a point $x_{0}=x$.

3. $H_{j k}(x)$ and its derivatives are bounded in a neighborhood of $x_{0}$.

Then we have

$$
\operatorname{tr}\left[H^{-1}\left(x_{0}\right) W^{-1}\left(x_{0}\right)\right] \leq(d-1)
$$

For qubits this bound can be attained by a particular estimation strategy which measures on each qubit separately. We refer to [66] for details.

A second quantity interesting to study in the limit $N \rightarrow \infty$ is the error probability defined in Section 4.2; cf. Equation (4.17). For a good estimation strategy it should go to zero of course, an additional question, however, concerns the rate with which this happens. We will review here a result from 99 which concerns the subproblem of estimating the spectrum. Hence we are looking now at a family of observables $E^{N}: \mathcal{C}\left(X_{N}\right) \rightarrow \mathcal{B}\left(\mathcal{H}^{\otimes N}\right), N \in \mathbb{N}$ taking their values in a finite subset $X_{N}$ of the set

$$
\Sigma=\left\{\left(x_{1}, \ldots, x_{d}\right) \in \mathbb{R}^{d} \mid x_{1} \geq \cdots \geq x_{d} \geq 0, \sum_{j} x_{j}=1\right\}
$$


of ordered spectra of density operators on $\mathcal{H}=\mathbb{C}^{d}$. Our aim is to determine the behavior of the error probabilities (cf. Equation (4.17)

$$
K_{N}(\Delta)=\sum_{x \in \Delta \cap X_{N}} \operatorname{tr}\left(E_{x}^{N} \rho^{\otimes N}\right)
$$

in the limit $N \rightarrow \infty$. Following the general arguments in Subsection 7.1 .2 we can restrict our attention here to covariant observables, i.e. we can assume without loss of cloning quality that the $E_{x}^{N}$ commute with all permutation unitaries $V_{p}, p \in \mathrm{S}_{N}$ and all local unitaries $U^{\otimes N}, U \in \mathrm{U}(d)$. If we restrict our attention in addition to projection valued measures, which is suggestive for ruling out unnecessary fuzziness, we see that each $E_{x}^{N}$ must coincide with a (sum of) projections $P_{Y}$ from $\mathcal{H}^{\otimes N}$ onto the $\mathrm{U}(d)$ respectively $V_{p}$ invariant subspace $\mathcal{H}_{Y} \otimes \mathcal{K}_{Y}$, which is defined in Equation (7.32), where $Y=\left(Y_{1}, \ldots, Y_{d}\right)$ refers here to Young frames with $d$ rows and $N$ boxes. The only remaining freedom for the $E^{N}$ is the assignment $x(Y) \in \Sigma$ of Young frames (and therefore projections $E_{N}$ ) to points in $\Sigma$. Since the Young frames themselves have up to normalization the same structure as the elements of $\Sigma$, one possibility for $s(Y)$ is just $s(Y)=Y / N$. Written as quantum to classical channel this is

$$
\mathcal{C}\left(X_{N}\right) \ni f \mapsto \sum_{Y} f(Y / N) P_{Y} \in \mathcal{B}\left(\mathcal{H}^{\otimes N}\right),
$$

where $X_{N} \subset \Sigma$ is the set of normalized Young frames, i.e. all $Y / N$ if $Y$ has $d$ rows and $N$ boxes. It turns out, somewhat surprisingly that this choice leads indeed to an asymptotically exact estimation strategy with exponentially decaying error probability (7.67). The following theorem can be proven with methods from the theory of large deviations:

TheOREm 7.3.2 The family of estimators $E^{N}, N \in \mathbb{N}$ given in Equation 7.68) is asymptotically exact, i.e. the error probabilities $K_{N}(\Delta)$ vanish in the limit $N \rightarrow \infty$ if $\Delta$ is a complement of a ball around the spectrum $r \in \Sigma$ of $\rho$. If $\Delta$ is a set (possibly containing $r$ ) whose interior is dense in its closure we have the asymptotic estimate for $K_{N}(\Delta)$ :

$$
\lim _{N \rightarrow \infty} \frac{1}{N} \ln K_{N}(\Delta)=\inf _{s \in \Delta} I(s),
$$

where the "rate function" $I: \Sigma \rightarrow \mathbb{R}$ is just the relative entropy between the two probability vectors $s$ and $r$

$$
I(s)=\sum_{j} s_{j}\left(\ln s_{j}-\ln r_{j}\right) .
$$

To make this statement more transparent, note that we can rephrase (7.69) as

$$
K_{N}(\Delta) \approx \exp \left(-N \inf _{s \in \Delta} I(s)\right)
$$

Since the rate function $I$ vanishes only for $s=r$ we see that the probability measures $K_{N}$ converge (weakly) to a point measure concentrated at $r \in \Sigma$. The rate of this convergence is exponential and measured exactly by the function $I$.

7.3.2. Purification and cloning. - Let us come back now to the discussion of purification started in Subsection 7.2 .2 (consequently we have $\mathcal{H}=\mathbb{C}^{2}$ again). Our aim is now to calculate the fidelities $\mathcal{F}_{R, \#}(N, M(N))$ in the limit $N \rightarrow \infty$ for a sequence $M(N), N \in \mathbb{N}$ such that $M(N) / N$ converges to a value $c \in \mathbb{R}$. The crucial 
step to do this is the application of Theorem 7.3.2. The density matrices $\rho_{s}(\beta)$ from Equation $(7.46)$ can be defined alternatively by

$$
\rho_{s}(\beta) \otimes \frac{\mathbb{I}}{\operatorname{dim} \mathcal{K}_{N, s}}=w_{N}(s)^{-1} P_{s} \rho(\beta)^{\otimes N} P_{s}, \quad w_{N}(s)=\operatorname{tr}\left(\rho(\beta)^{\otimes N} P_{s}\right)
$$

where $P_{s}$ is the projection from $\mathcal{H}^{\otimes N}$ to $\mathcal{H}_{s} \otimes \mathcal{K}_{N, s}$. In other words $P_{s}$ is equal to $P_{Y}$ from Equation (7.68) if we apply the reparametrization

$$
\left(Y_{1}, Y_{2}\right) \mapsto(s, N)=\left(\left(Y_{1}-Y_{2}\right) / 2, Y_{1}+Y_{2}\right) .
$$

In a similar way we can rewrite the set of ordered spectra by $\Sigma \ni\left(x_{1}, x_{2}\right) \mapsto$ $x_{1}-x_{2} \in[0,1]$ and $K_{N}(\Delta)$ becomes a measure on $[0,1]($ i.e. $\Delta \subset[0,1])$ :

$$
K_{N}(\Delta)=\sum_{2 s / N \in \Delta} \operatorname{tr}\left(\rho(\beta)^{\otimes N} P_{s}\right)=\sum_{2 s / N \in \Delta} w_{N}(s)
$$

and the sum

$$
\mathcal{F}_{R, \#}(N, M(N))=\sum_{s} w_{N}(s) f_{\#}(M(N), \beta, s)
$$

can be rephrased as the integral of a function $[0,1] \ni x \mapsto \widetilde{f}_{\#}(N, \beta, x) \in \mathbb{R}$ with respect to this measure, provided $\widetilde{f}_{\#}$ is related to $f_{\#}$ by $\widetilde{f}_{\#}(N, \beta, 2 s / N)=$ $f_{\#}(M(N), \beta, s)$. According to Theorem 7.3.2 the $K_{N}$ converge to a point measure concentrated at the ordered spectrum of $\rho(\beta)$; but the latter corresponds, according to the reparametrization above, to the noise parameter $\vartheta=\tanh \beta$. Hence if the sequence of functions $\widetilde{f}_{\#}(N, \beta, \cdot)$ converges for $N \rightarrow \infty$ uniformly (or at least uniformly on a neighborhood of $\vartheta)$ to $\widetilde{f}_{\#}(\beta, \cdot)$ we get

$$
\lim _{N \rightarrow \infty} \mathcal{F}(N, M(N))=\lim _{N \rightarrow \infty} \sum_{s} \widetilde{f}_{\#}(N, \beta, s)=\widetilde{f}_{\#}(\beta, \vartheta)
$$

for the limit of the fidelities. A precise formulation of this idea leads to the following theorem 100

TheOREM 7.3.3 The two purification fidelities $\mathcal{F}_{R \text {,\# }}$ have the following limits

$$
\lim _{N \rightarrow \infty} \lim _{M \rightarrow \infty} \mathcal{F}_{R, 1}(N, M)=1
$$

and

$$
\Phi(\mu)=\lim _{\substack{N \rightarrow \infty \\ M / N \rightarrow \mu}} \mathcal{F}_{R, \text { all }}(N, M)= \begin{cases}\frac{2 \vartheta^{2}}{2 \vartheta^{2}+\mu(1-\vartheta)} & \text { if } \mu \leq \vartheta \\ \frac{2 \vartheta^{2}}{\mu(1+\vartheta)} & \text { if } \mu \geq \vartheta .\end{cases}
$$

If we are only interested in the quality of each qubit separately we can produce arbitrarily good purified qubits at any rate. If on the other hand the correlations between the output systems should vanish in the limit the rate is always zero. This can be seen from the function $\Phi$, which is the asymptotic all-qubit fidelity which can be reached by a given rate $\mu$. We have plotted it in Figure 7.4. Note finally that the results just stated contain the rates of optimal cloning machines as a special case; we only have to set $\vartheta=1$. 


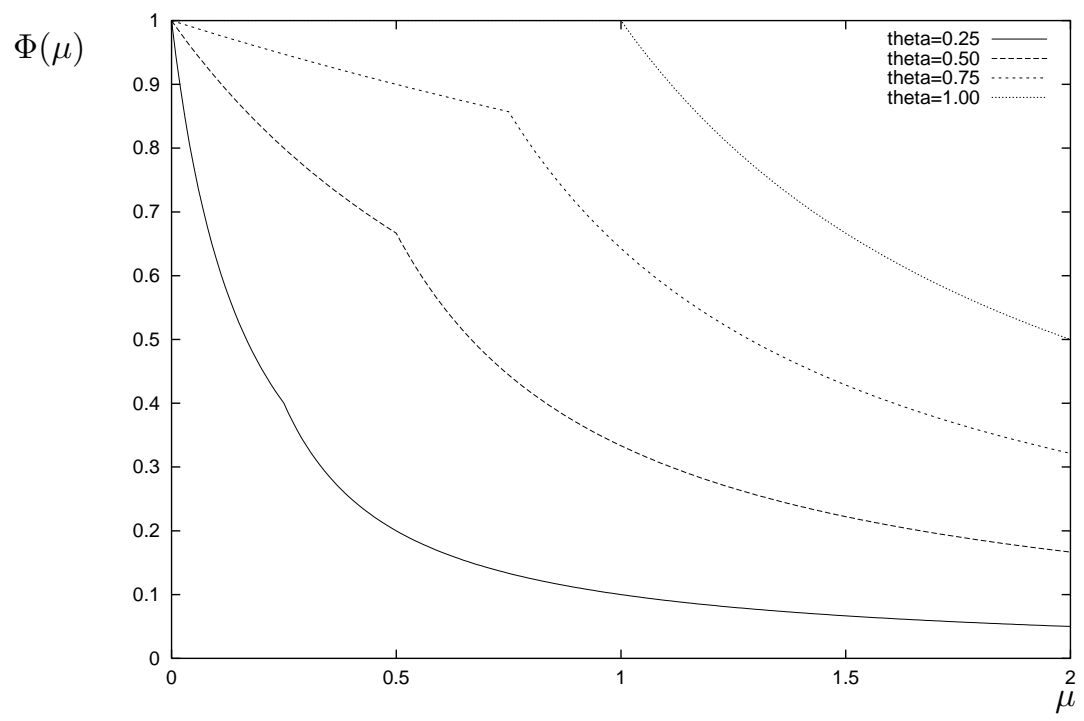

Figure 7.4: Asymptotic all-qubit fidelity $\Phi(\mu)$ plotted as function of the rate $\mu$. 


\section{Bibliography}

[1] A. Acín, A. Andrianov, L. Costa, E. Jané, J. I. Latorre and R. Tarrach. Schmidt decomposition and classification of three-quantum-bit states. Phys. Rev. Lett. 85, no. 7, 1560-1563 (2000).

[2] C. Adami and N. J. Cerf. Von Neumann capacity of noisy quantum channels. Phys. Rev. A 56, no. 5, 3470-3483 (1997).

[3] G. Alber, T. Beth, M. Horodecki, R. Horodecki, M. Rötteler, H. Weinfurter, R. Werner and A. Zeilinger (editors). Quantum information. Springer, Berlin (2001).

[4] A. Ashikhmin and E. Knill. Nonbinary quantum stabilizer codes. IEEE T. Inf. Theory 47, no. 7, 3065-3072 (2001).

[5] A. Aspect, J. Dalibard and G. Roger. Experimental test of Bell's inequalities using time-varying analyzers. Phys. Rev. Lett. 49, 1804-1807 (1982).

[6] H. Barnum, E. Knill and M. A. Nielsen. On quantum fidelities and channel capacities. IEEE Trans. Inf. Theory 46, 1317-1329 (2000).

[7] H. Barnum, M. A. Nielsen and B. Schumacher. Information transmission through a noisy quantum channel. Phys. Rev. A 57, no. 6, 4153-4175 (1998).

[8] H. Barnum, J. A. Smolin and B. M. Terhal. Quantum capacity is properly defined without encodings. Phys. Rev A 58, no. 5, 3496-3501 (1998).

[9] C. H. Bennett, H. J. Bernstein, S. Popescu and B. Schumacher. Concentrating partial entanglement by local operations. Phys. Rev. A 53, no. 4, 2046-2052 (1996).

[10] C. H. Bennett and G. Brassard. Quantum key distribution and coin tossing. In Proc. of IEEE Int. Conf. on Computers, Systems, and Signal Processing (Bangalore, India, 1984), pages 175-179. IEEE, New York (1984).

[11] C. H. Bennett, G. Brassard, C. Crépeau, R. Jozsa, A. Peres and W. K. Wootters. Teleporting an unknown quantum state via dual classical and EinsteinPodolsky-Rosen channels. Phys. Rev. Lett. 70, 1895-1899 (1993).

[12] C. H. Bennett, G. Brassard, S. Popescu, B. Schumacher, J. A. Smolin and W. K. Wootters. Purification of noisy entanglement and faithful teleportation via noisy channels. Phys. Rev. Lett. 76, no. 5, 722-725 (1996). Erratum: Phys. Rev. Lett. 78, 10, 2031 (1997).

[13] C. H. Bennett, D. P. DiVincenzo, C. A. Fuchs, T. Mor, E. M. Rains, P. W. Shor, J. A. Smolin and W. K. Wootters. Quantum nonlocality without entanglement. Phys. Rev. A 59, no. 2, 1070-1091 (1999).

[14] C. H. Bennett, D. P. DiVincenzo, T. Mor, P. W. Shor, J. A. Smolin and B. M. Terhal. Unextendible product bases and bound entanglement. Phys. Rev. Lett 82, no. 26, 5385-5388 (1999).

[15] C. H. Bennett, D. P. DiVincenzo and J. A. Smolin. Capacities of quantum erasure channels. Phys. Rev. Lett. 78, no. 16, 3217-3220 (1997). 
[16] C. H. Bennett, D. P. DiVincenzo, J. A. Smolin and W. K. Wootters. Mixedstate entanglement and quantum error correction. Phys. Rev. A 54, no. 4, 3824-3851 (1996).

[17] C. H. Bennett, P. W. Shor, J. A. Smolin and A. V. Thapliyal. Entanglementassisted classical capacity of noisy quantum channels. Phys. Rev. Lett. 83, no. 15, 3081-3084 (1999).

[18] C. H. Bennett, P. W. Shor, J. A. Smolin and A. V. Thapliyal. Entanglementassisted capacity of a quantum channel and the reverse Shannon theorem. quant-ph/0106052 (2001).

[19] C. H. Bennett and S. J. Wiesner. Communication via one- and two-particle operators on Einstein-Podolsky-Rosen states. Phys. Rev. Lett. 20, 2881-2884 (1992).

[20] T. Beth and M. Rötteler. Quantum algorithms: Applicable algebra and quantum physics. In Quantum information ( G. Alber et. al., editor), pages 97-150. Springer (2001).

[21] E. Biolatti, R. C. Iotti, P. Zanardi and F. Rossi. Quantum information processing with semiconductor macroatoms. Phys. Rev. Lett. 85, no. 26, 5647-5650 (2000).

[22] D. Boschi, S. Branca, F. De Martini, L. Hardy and S. Popescu. Experimental realization of teleporting an unknown pure quantum state via dual classical an Einstein-Podolsky-Rosen channels. Phys. Rev. Lett. 80, no. 6, 1121-1125 (1998).

[23] D. Bouwmeester, A. K. Ekert and A. Zeilinger (editors). The physics of quantum information: Quantum cryptography, quantum teleportation, quantum computation. Springer, Berlin (2000).

[24] D. Bouwmeester, J.-W. Pan, K. Mattle, M. Eibl, H. Weinfurter and A. Zeilinger. Experimental quantum teleportation. Nature 390, 575-579 (1997).

[25] O. Bratteli and D. W. Robinson. Operator Algebras and Quantum Statistical Mechanics. I. Springer, New York (1979).

[26] O. Bratteli and D. W. Robinson. Operator Algebras and Quantum Statistical Mechanics II. Springer, Berlin (1997).

[27] S. L. Braunstein, C. M. Caves, R. Jozsa, N. Linden, S. Popescu and R. Schack. Separability of very noisy mixed states and implications for NMR quantum computing. Phys. Rev. Lett. 83, no. 5, 1054-1057 (1999).

[28] G. K. Brennen, C. M. Caves and I. H. Deutsch F. S. Jessen. Quantum logic gates in optical lattices. Phys. Rev. Lett. 82, no. 5, 1969-1063 (1999).

[29] K.R. Brown, D.A. Lidar and K.B. Whaley. Quantum computing with quantum dots on linear supports. quant-ph/0105102 (2001).

[30] T. A. Brun and H. L. Wang. Coupling nanocrystals to a high-q silica microsphere: Entanglement in quantum dots via photon exchange. Phys. Rev. A 61, 032307 (2000).

[31] D. Bruß, D. P. DiVincenzo, A. Ekert, C. A. Fuchs, C. Machiavello and J. A. Smolin. Optimal universal and state-dependent cloning. Phys. Rev. A 57, no. 4, 2368-2378 (1998). 
[32] D. Bruß, A. K. Ekert and C. Macchiavello. Optimal universal quantum cloning and state estimation. Phys. Rev. Lett. 81, no. 12, 2598-2601 (1998).

[33] D. Bruß and C. Macchiavello. Optimal state estimation for d-dimensional quantum systems. Phys. Lett. A253, 249-251 (1999).

[34] W. T. Buttler, R.J. Hughes, S.K. Lamoreaux, G.L. Morgan, J.E. Nordholt and C.G. Peterson. Daylight quantum key distribution over $1.6 \mathrm{~km}$. Phys. Rev. Lett 84, 5652-5655 (2000).

[35] V. Bužek and M. Hillery. Universal optimal cloning of qubits and quantum registers. Phys. Rev. Lett. 81, no. 22, 5003-5006 (1998).

[36] V. Bužek, M. Hillery and R. F. Werner. Optimal manipulations with qubits: Universal-not gate. Phys. Rev. A 60, no. 4, R2626-R2629 (1999).

[37] A. Cabello. Bibliographic guide to the foundations of quantum mechanics and quantum information. quant-ph/0012089 (2000).

[38] A. R. Calderbank, E. M. Rains, P. W. Shor and N. J. A. Sloane. Quantum error correction and orthogonal geometry. Phys. Rev. Lett. 78, no. 3, 405-408 (1997).

[39] A. R. Calderbank and P. W. Shor. Good quantum error-correcting codes exist. Phys. Rev. A 54, 1098-1105 (1996).

[40] N. J. Cerf. Asymmetric quantum cloning machines. J.Mod.Opt. 47, 187(2000).

[41] N. J. Cerf and C. Adami. Negative entropy and information in quantum mechanics. Phys. Rev. Lett. 79, no. 26, 5194-5197 (1997).

[42] N. J. Cerf, C. Adami and R. M. Gingrich. Reduction criterion for separability. Phys. Rev. A 60, no. 2, 898-909 (1999).

[43] N. J. Cerf, S. Iblisdir and G. van Assche. Cloning and cryptography with quantum continuous variables. quant-ph/0107077 (2001).

[44] I. L. Chuang, L. M. K. Vandersypen, X. L. Zhou, D. W. Leung and S. Lloyd. Experimental realization of a quantum algorithm. Nature 393, 143-146 (1998).

[45] A. Church. An unsolved problem of elementary number theory. Amer. J. Math. 58, 345-363 (1936).

[46] J. I. Cirac, A. K. Ekert and C. Macchiavello. Optimal purification of single qubits. Phys. Rev. Lett. 82, 4344-4347 (1999).

[47] J. F. Clauser, M. A. Horne, A. Shimony and R. A. Holt. Proposed experiment to test local hidden-variable theories. Phys. Rev. Lett 23, no. 15, 880-884 (1969).

[48] J. F. Cornwell. Group theory in physics. II. Academic Press, London et. al. (1984).

[49] T. M. Cover and J. A. Thomas. Elements of information theory. Wiley, Chichester (1991).

[50] E. B. Davies. Quantum Theory of Open Systems. Academic Press, London (1976). 
[51] B. Demoen, P. Vanheuverzwijn and A. Verbeure. Completely positive maps on the CCR-algebra. Lett. Math. Phys. 2, 161-166 (1977).

[52] D. Deutsch. Quantum theory, the Church-Turing principle and the universal quantum computer. Proc. R. Soc. Lond. A 400, 97-117 (1985).

[53] D. Deutsch and R. Jozsa. Rapid solution of problems by quantum computation. Proc. R. Soc. Lond. A 439, 553-558 (1992).

[54] D. P. DiVincenzo, P. W. Shor and J. A. Smolin. Quantum-channel capacity of very noisy channels. Phys. Rev. A 57, no. 2, 830-839 (1998). Erratum: Phys. Rev. A 59, 2, 1717 (1999).

[55] D.P. DiVincenzo, P.W. Shor, J.A. Smolin, B.M. Terhal and A.V. Thapliyal. Evidence for bound entangled states with negative partial transpose. Phys. Rev. A 61, no. 6, 062312 (2000).

[56] M. J. Donald and M. Horodecki. Continuity of relative entropy of entanglement. Phys. Lett A 264, no. 4, 257-260 (1999).

[57] M. J. Donald, M. Horodecki and O. Rudolph. The uniqueness theorem for entanglement measures. quant-ph/0105017 (2001).

[58] W. Dür, J.I. Cirac, M. Lewenstein and D. Bruss. Distillability and partial transposition in bipartite systems. Phys. Rev. A 61, no. 6, 062313 (2000).

[59] B. Efron and R. J. Tibshirani. An introduction to the bootstrap. Chapman and Hall, New York (1993).

[60] T. Eggeling, K. G. H. Vollbrecht, R. F. Werner and M. M. Wolf. Distillability via protocols respecting the positivity of the partial transpose. Phys. Rev. Lett. 87, 257902 (2001).

[61] T. Eggeling and R. F. Werner. Separability properties of tripartite states with $U \times U \times U$-symmetry. Phys. Rev. A 63, no. 4, 042111 (2001).

[62] A. Feinstein. Foundations of Informations Theory. McGraw-Hill, New York (1958).

[63] D. G. Fischer and M. Freyberger. Estimating mixed quantum states. Phys. Lett. A273, 293-302 (2000).

[64] G. Giedke, L.-M. Duan, J. I. Cirac and P. Zoller. Distillability criterion for all bipartite gaussian states. Quant. Inf. Comp. 1, no. 3 (2001).

[65] G. Giedke, B. Kraus, M. Lewenstein and J. I. Cirac. Separability properties of three-mode gaussian states. Phys. Rev. A 64, no. 05, 052303 (2001).

[66] R. D. Gill and S. Massar. State estimation for large ensembles. Phys. Rev. A61, 2312-2327 (2000).

[67] N. Gisin. Hidden quantum nonlocality revealed by local filters. Phys. Lett. A 210, no. 3, 151-156 (1996).

[68] N. Gisin and S. Massar. Optimal quantum cloning machines. Phys.Rev.Lett. 79, no. 11, 2153-2156 (1997).

[69] N. Gisin, G. Ribordy, W. Tittel and H. Zbinden. Quantum cryptography. quant-ph/0101098 (2001). 
[70] D. Gottesman. Class of quantum error-correcting codes saturating the quantum hamming bound. Phys. Rev. A 54, 1862-1868 (1996).

[71] D. Gottesman. Stabilizer codes and quantum error correction. Ph.D. thesis, California Institute of Technology (1997). quant-ph/9705052.

[72] M. Grassl, T. Beth and T. Pellizzari. Codes for the quantum erasure channel. Phys. Rev. A 56, no. 1, 33-38 (1997).

[73] D. M. Greenberger, M. A. Horne and A. Zeilinger. Going beyond bell's theorem. In Bell's theorem, quantum theory, and conceptions of the universe ( M. Kafatos, editor), pages 69-72. Kluwer Academic, Dordrecht (1989).

[74] L. K. Grover. Quantum computers can search arbitrarily large databases by a single query. Phys. Rev. A 56, no. 23, 4709-4712 (1997).

[75] L. K. Grover. Quantum mechanics helps in searching for a needle in a haystack. Phys. Rev. Lett. 79, no. 2, 325-328 (1997).

[76] J. Gruska. Quantum computing. McGraw-Hill, New York (1999).

[77] Jim Harrington and John Preskill. Achievable rates for the gaussian quantum channel. Phys. Rev. A 64, no. 6, 062301 (2001).

[78] P. M. Hayden, M. Horodecki and B. M. Terhal. The asymptotic entanglement cost of preparing a quantum state. J. Phys. A., Math. Gen. 34, no. 35, 68916898 (2001).

[79] A. S. Holevo. Probabilistic and statistical aspects of quantum theory. NorthHolland, Amsterdam (1982).

[80] A. S. Holevo. Coding theorems for quantum channels. Tamagawa University Research Review no. 4 (1998). quant-ph/9809023.

[81] A. S. Holevo. Sending quantum information with gaussian states. In Proc. of the 4 th Int. Conf. on Quantum Communication, Measurement and Computing (Evanston, 1998) (1998). quant-ph/9809022.

[82] A. S. Holevo. On entanglement-assisted classical capacity. quant-ph/0106075 (2001).

[83] A. S. Holevo. Statistical structure of quantum theory. Springer, Berlin (2001).

[84] A. S. Holevo and R. F. Werner. Evaluating capacities of bosonic gaussian channels. Phys. Rev. A 63, no. 3, 032312 (2001).

[85] M. Horodecki and P. Horodecki. Reduction criterion of separability and limits for a class of distillation protocols. Phys. Rev. A 59, no. 6, 4206-4216 (1999).

[86] M. Horodecki, P. Horodecki and R. Horodecki. Separability of mixed states: Necessary and sufficient conditions. Phys. Lett. A 223, no. 1-2, 1-8 (1996).

[87] M. Horodecki, P. Horodecki and R. Horodecki. Mixed-state entanglement and distillation: Is there a "bound" entanglement in nature? Phys. Rev. Lett. 80, no. 24, 5239-5242 (1998).

[88] M. Horodecki, P. Horodecki and R. Horodecki. General teleportation channel, singlet fraction, and quasidistillation. Phys. Rev. A 60, no. 3, 1888-1898 (1999). 
[89] M. Horodecki, P. Horodecki and R. Horodecki. Limits for entanglement measures. Phys. Rev. Lett. 84, no. 9, 2014-2017 (2000).

[90] M. Horodecki, P. Horodecki and R. Horodecki. Unified approach to quantum capacities: Towards quantum noisy coding theorem. Phys. Rev. Lett. 85, no. 2, 433-436 (2000).

[91] M. Horodecki, P. Horodecki and R. Horodecki. Mixed-state entanglement and quantum communication. In Quantum information ( G. Alber et. al., editor), pages 151-195. Springer (2001).

[92] P. Horodecki, M. Horodecki and R. Horodecki. Bound entanglement can be activated. Phys. Rev. Lett. 82, no. 5, 1056-1059 (1999).

[93] R. J. Hughes, G. L. Morgan and C. G. Peterson. Quantum key distribution over a $48 \mathrm{~km}$ optical fibre network. J. Mod. Opt. 47, no. 2-3, 533-547 (2000).

[94] A. Jamiołkowski. Linear transformations which preserve trace and positive semidefiniteness of operators. Rep. Math. Phys. 3, 275-278 (1972).

[95] T. Jennewein, C. Simon, G. Weihs, H. Weinfurter and A. Zeilinger. Quantum cryptography with entangled photons. Phys. Rev. Lett. 84, 4729-4732 (2000).

[96] J. A. Jones, M. Mosca and R. H. Hansen. Implementation of a quantum search algorithm on a quantum computer. Nature 393, 344-346 (1998).

[97] M. Keyl, D. Schlingemann and R. F. Werner. Infinitely entangled states. in preparation.

[98] M. Keyl and R. F. Werner. Optimal cloning of pure states, testing single clones. J. Math. Phys. 40, 3283-3299 (1999).

[99] M. Keyl and R. F. Werner. Estimating the spectrum of a density operator. Phys. Rev. A 64, no. 5, 052311 (2001).

[100] M. Keyl and R. F. Werner. The rate of optimal purification procedures. Ann H. Poincaré 2, 1-26 (2001).

[101] A. I. Khinchin. Mathematical Foundations of Information Theory. Dover Publications, New York (1957).

[102] B. E. King, C. S. Wood, C. J. Myatt, Q. A. Turchette, D. Leibfried, W. M. Itano, C. Monroe and D. J. Wineland. Cooling the collective motion of trapped ions to initialize a quantum register. Phys. Rev. Lett 81, no. 7, 1525-528 (1998).

[103] E. Knill and R. Laflamme. Theory of quantum error-correcting codes. Phys. Rev. A 55, no. 2, 900-911 (1997).

[104] B. Kraus, M. Lewenstein and J. I. Cirac. Characterization of distillable and activable states using entanglement witnesses. quant-ph/0110174 (2001).

[105] K. Kraus. States effects and operations. Springer, Berlin (1983).

[106] R. Landauer. Irreversibility and heat generation in the computing process. IBM J. Res. Dev. 5, 183 (1961).

[107] U. Leonhardt. Measuring the quantum state of light. Cambridge Univ. Press, Cambridge (1997). 
[108] M. Lewenstein and A. Sanpera. Separability and entanglement of composite quantum systems. Phys. Rev. Lett. 80, no. 11, 2261-2264 (1998).

[109] N. Linden, H. Barjat and R. Freeman. An implementation of the DeutschJozsa algorithm on a three-qubit NMR quantum computer. Chem. Phys. Lett. 296, no. 1-2, 61-67 (1998).

[110] S. Lloyd. Capacity of the noisy quantum channel. Phys. Rev. A 55, no. 3, 1613-1622 (1997).

[111] H.-K. Lo and and T. Spiller S. Popescu (editors). Introduction to quantum computation and information. World Scientific, Singapore (1998).

[112] Y. Makhlin, G. Schön and A. Shnirman. Quantum-state engineering with Josephson-junction devices. Rev. Mod. Phys. 73, no. 2, 357-400 (2001).

[113] R. Marx, A. F. Fahmy, J. M. Myers, W. Bermel and S. J. Glaser. Approaching five-bit NMR quantum computing. Phys. Rev. A 62, no. 1, 012310 (2000).

[114] R. Matsumoto and T. Uyematsu. Lower bound for the quantum capacity of a discrete memoryless quantum channel. quant-ph/0105151 (2001).

[115] K. Mattle, H. Weinfurter, P. G. Kwiat and A. Zeilinger. Dense coding in experimental quantum communication. Phys. Rev. Lett. 76, no. 25, 46564659 (1996).

[116] N. D. Mermin. Quantum mysteries revisited. Am. J. Phys. 58, no. 8, 731-734 (1990).

[117] N. D. Mermin. What's wrong with these elements of reality? Phys. Today 43, no. 6, 9-11 (1990).

[118] H. C. Nagerl, W. Bechter, J. Eschner, F. Schmidt-Kaler and R. Blatt. Ion strings for quantum gates. Appl. Phys. B 66, no. 5, 603-608 (1998).

[119] M. A. Nielsen. Conditions for a class of entanglement transformations. Phys. Rev. Lett. 83, no. 2, 436-439 (1999).

[120] M. A. Nielsen. Continuity bounds for entanglement. Phys. Rev. A 61, no. 6, 064301 (2000).

[121] M. A. Nielsen. Characterizing mixing and measurement in quantum mechanics. Phys. Rev. A 63, no. 2, 022114 (2001).

[122] M. A. Nielsen and I. L. Chuang. Quantum computation and quantum information. Cambridge University Press, Cambridge (2000).

[123] M. Ohya and D. Petz. Quantum entropy and its use. Springer, Berlin (1993).

[124] C. M. Papadimitriou. Computational complexity. Addison-Wesley, Reading, Massachusetts (1994).

[125] V. I. Paulsen. Completely bounded maps and dilations. Longman Scientific \& Technical (1986).

[126] A. Peres. Higher order schmidt decompositions. Phys. Lett. A 202, no. 1, 16-17 (1995).

[127] A. Peres. Separability criterion for density matrices. Phys. Rev. Lett. 77, no. 8, 1413-1415 (1996). 
[128] S. Popescu. Bell's inequalities versus teleportation: What is nonlocality? Phys. Rev. Lett. 72, no. 6, 797-799 (1994).

[129] S. Popescu and D. Rohrlich. Thermodynamics and the measure of entanglement. Phys. Rev. A 56, no. 5, R3319-R3321 (1997).

[130] J. Preskill. Lecture notes for the course 'information for physics 219/computer science 219, quantum computation'. Caltech, Pasadena, California (1999). www.theory.caltech.edu/people/preskill/ph229.

[131] M. Purser. Introduction to error-correcting codes. Artech House, Boston (1995).

[132] E. M. Rains. Bound on distillable entanglement. Phys. Rev. A 60, no. 1, 179-184 (1999). Erratum: Pys. Rev. A 63, 1, 019902(E) (2001).

[133] E. M. Rains. A semidefinite program for distillable entanglement. IEEE T. Inf. Theory 47, no. 7, 2921-2933 (2001).

[134] M. Reed and B. Simon. Methods of modern mathematical physics. I. Academic Press, San Diego (1980).

[135] W. Rudin. Functional Analysis. McGraw-Hill, New-York (1973).

[136] O. Rudolph. A separability criterion for density operators. J. Phys. A 33, no. $21,3951-3955$ (2000).

[137] D. Schlingemann and R. F. Werner. Quantum error-correcting codes associated with graphs. quant-ph/0012111 (2000).

[138] C. E. Shannon. A mathematical theory of communication. Bell. Sys. Tech. J. 27, 379-423, 623-656 (1948).

[139] P. W. Shor. Algorithms for quantum computation: Discrete logarithms and factoring. In Proc. of the 35th Annual Symposium on the Foundations of Computer Science ( S. Goldwasser, editor), pages 124-134. IEEE Computer Science, Society Press, Los Alamitos, California (1994).

[140] P. W. Shor. Polynomial-time algorithms for prime factorization and discrete logarithms on a quantum computer. Soc. Ind. Appl. Math. J. Comp. 26, 1484-1509 (1997).

[141] P. W. Shor, J. A. Smolin and B. M. Terhal. Nonadditivity of bipartite distillable entanglement follows from a conjecture on bound entangled Werner states. Phys. Rev. Lett. 86, no. 12, 2681-2684 (2001).

[142] B. Simon. Representations of finite and compact groups. American Mathematical Society, Providence (1996).

[143] D. Simon. On the power of quantum computation. In Proc. 35th annual symposium on foundations of computer science, pages 124-134. IEEE Computer Society Press, Los Alamitos (1994).

[144] R. Simon. Peres-Horodecki separability criterion for continuous variable systems. Phys. Rev. Lett. 84, no. 12, 2726-2729 (2000).

[145] S. Singh. The code book: The Science of Secrecy from Ancient Egypt to Quantum Cryptography. Fourh Estate, London (1999). 
[146] A. M. Steane. Multiple particle interference and quantum error correction. Proc. Roy. Soc. Lond. A 452, 2551-2577 (1996).

[147] W. F. Stinespring. Positive functions on $C^{*}$-algebras. Proc. Amer. Math. Soc. pages 211-216 (1955).

[148] E. Størmer. Positive linear maps of operator algebras. Acta Math. 110, 233278 (1693).

[149] T. Tanamoto. Quantum gates by coupled asymmetric quantum dots and controlled-not-gate operation. Phys. Rev. A 61, 022305 (2000).

[150] B. M. Terhal and K. G. H. Vollbrecht. Entanglement of formation for isotropic states. Phys. Rev. Lett. 85, no. 12, 2625-2628 (2000).

[151] W. Tittel, J. Brendel and H. Zbinden N. Gisin. Violation of Bell inequalities by photons more than $10 \mathrm{~km}$ apart. Phys. Rev. Lett. 81, no. 17, 3563-3566 (1998).

[152] A. M. Turing. On computable numbers, with an application to the entscheidungsproblem. Proc. Lond. Math. Soc. Ser. 2 42, 230-265 (1936).

[153] V. Vedral and M. B. Plenio. Entanglement measures and purification procedures. Phys. Rev. A 54, no. 3, 1619-1633 (1998).

[154] V. Vedral, M. B. Plenio, M. A. Rippin and P. L. Knight. Quantifying entanglement. Phys. Rev. Lett. 78, no. 12, 2275-2279 (1997).

[155] G. Vidal. Entanglement monotones. J. Mod. Opt. 47, no. 2-3, 355-376 (2000).

[156] G. Vidal, J. I. Latorre, P. Pascual and R. Tarrach. Optimal minimal measurements of mixed states. Phys. Rev. A60, 126-135 (1999).

[157] G. Vidal and R. Tarrach. Robustness of entanglement. Phys. Rev. A 59, no. 1, 141-155 (1999).

[158] G. Vidal and R. F. Werner. A computable measure of entanglement. quant$\mathrm{ph} / 0102117$ (2001).

[159] K. G. H. Vollbrecht and R. F. Werner. Entanglement measures under symmetry. quant-ph/0010095. (2000).

[160] K. G. H. Vollbrecht and R. F. Werner. Why two qubits are special. J. Math. Phys. 41, no. 10, 6772-6782 (2000).

[161] I. Wegener. The complexity of boolean functions. Teubner, Stuttgart (1987).

[162] S. Weigert. Reconstruction of quantum states and its conceptual implications. In Trends in quantum mechanics ( H. D. Doebner, S. T. Ali, M. Keyl and R. F. Werner, editors), pages 146-156. World Scientific, Singapore (2000).

[163] H. Weinfurter and A. Zeilinger. Quantum communication. In Quantum information ( G. Alber et. al., editor), pages 58-95. Springer (2001).

[164] R. Werner and M. M. Wolf. Bell inequalities and entanglement. Quant. Inf. Comp. 1, no. 3, 1-25 (2001).

[165] R. F. Werner. Quantum harmonic analysis on phase space. J. Math. Phys. 25, 1404-1411 (1984). 
[166] R. F. Werner. Quantum states with Einstein-Podolsky-Rosen correlations admitting a hidden-variable model. Phys. Rev. A 40, no. 8, 4277-4281 (1989).

[167] R. F. Werner. Optimal cloning of pure states. Phys.Rev. A 58, 980-1003 (1998).

[168] R. F. Werner. All teleportation and dense coding schemes. quant-ph/0003070 (2000).

[169] R. F. Werner. Quantum information theory - an invitation. In Quantum information ( G. Alber et. al., editor), pages 14-59. Springer (2001).

[170] R. F. Werner and M. M. Wolf. Bound entangled gaussian states. Phys. Rev. Lett. 86, no. 16, 3658-3661 (2001).

[171] H. Weyl. The classical groups. Princeton University, Princeton (1946).

[172] W. K. Wooters. Entanglement of formation of an arbitrary state of two qubits. Phys. Rev. Lett. 80, no. 10, 2245-2248 (1998).

[173] W. K. Wootters and W. H. Zurek. A single quantum cannot be cloned. Nature 299, 802-803 (1982).

[174] S. L. Woronowicz. Positive maps of low dimensional matrix algebras. Rep. Math. Phys. 10, 165-183 (1976). 